\title{
Synthesis of Swinhoeisterol A, Dankasterone A and B, and Periconiastone A by Radical Framework Reconstruction
}

\author{
Fenja L. Duecker, Robert C. Heinze, Philipp Heretsch* \\ Institute of Chemistry and Biochemistry, Freie Universität Berlin, Takustraße 3, 14195 Berlin, \\ Germany \\ *Correspondence to: philipp.heretsch@fu-berlin.de
}

\section{Supporting Information}

Table of Contents

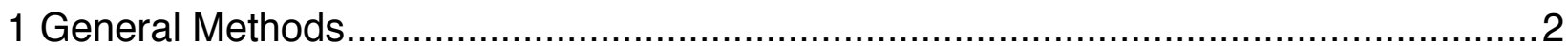

2 Experimental Procedures and Characterization Data ............................................... 3

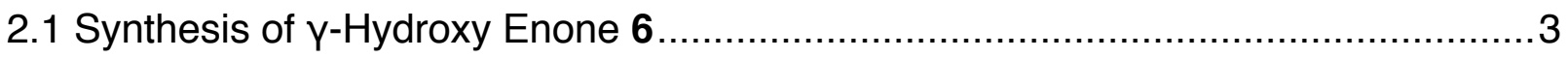

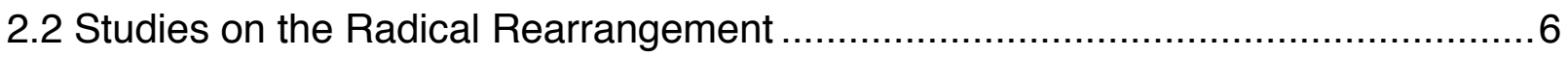

2.3 Synthesis of Dankasterone A ( $\left.\Delta^{4}-2\right)$ and B (2), and Periconiastone A (3) ...........10

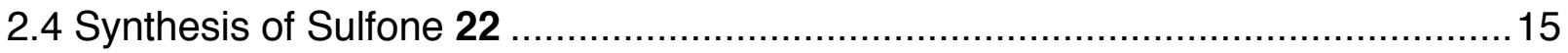

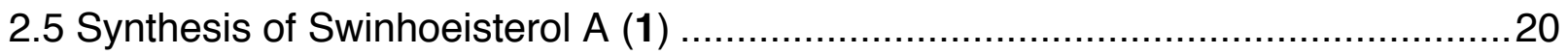

2.6 Synthesis of 24-epi-Swinhoeisterol A (24-epi-1) ................................................. 45

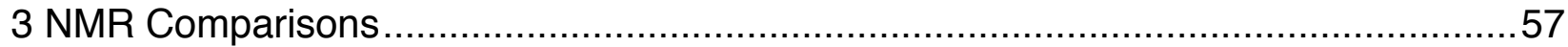

4 NMR Spectra

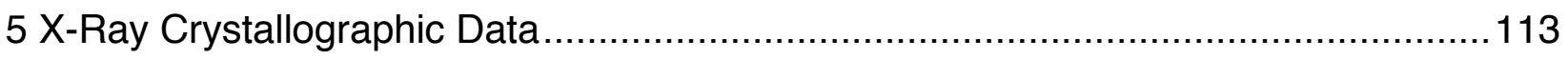

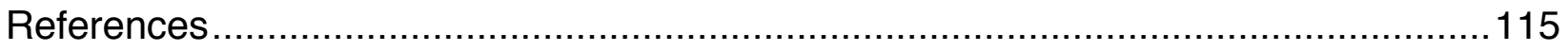




\section{General Methods}

All reactions sensitive to moisture and/or air were carried out using heat gun dried glassware, an argon atmosphere, and dry solvents. Dry dichloromethane, toluene, and $\mathrm{Et}_{2} \mathrm{O}$ were taken from a $\mathrm{M}$. Braun $\mathrm{GmbH}$ MB SPS-800 solvent purification system. THF was distilled from sodium and stored over $4 \AA$ molecular sieves. Triethylamine was distilled from $\mathrm{CaH}_{2}$ and stored over $\mathrm{KOH}$. Ethyl acetate and nhexane were purified by distillation on a rotary evaporator. All other solvents and commercially available reagents were used without further purification unless otherwise stated. Reactions were monitored by thin-layer chromatography (TLC) carried out on Merck Silica Gel 50 F245-plates and visualized by fluorescence quenching under UVlight or an aqueous solution of cerium sulfate and phosphomolybdic acid and heat as developing agent. Column chromatographic purification was performed on Macherey-Nagel Silica Gel $60 \mathrm{M}(40-60 \mu \mathrm{m})$. HPLC separation was performed on a Knauer system with DAD detection at $254 \mathrm{~nm}$. Concentration under reduced pressure was performed by rotary evaporation at $45^{\circ} \mathrm{C}$ and appropriate pressure, followed by exposure to high vacuum (10-3 mbar) at $25^{\circ} \mathrm{C}$. NMR spectra were recorded on either a Jeol ECX400 (400 MHz), a Jeol ECP500 (500 MHz), a Bruker AVANCE III $500(500 \mathrm{MHz})$, a Varian INOVA600 (600 MHz) or a Bruker AVANCE III 700 (700 MHz, with CryoProbe) spectrometer. Chemical shifts $\delta$ are reported in parts per million (ppm) and are referenced using residual undeuterated solvent $\left(\mathrm{CDCl}_{3}: \delta_{\mathrm{H}}=7.26 \mathrm{ppm}, \delta_{\mathrm{C}}=77.16 \mathrm{ppm}\right.$; pyridine- $d_{5}: \delta_{H}=8.74 \mathrm{ppm}, \delta_{\mathrm{C}}=150.35 \mathrm{ppm}$; unless otherwise stated) as an internal reference at $298 \mathrm{~K}$. The given multiplicities are phenomenological; thus the actual appearance of the signals is stated and not the theoretically expected one. The following abbreviations are used to designate multiplicities: $s=$ singlet, $\mathrm{d}=$ doublet, $\mathrm{t}=$ triplet, $\mathrm{q}=$ quartet, $\mathrm{p}=$ pentet, $\mathrm{br}=$ broad, and combinations thereof. In case no multiplicity could be identified, the chemical shift range of the signal is given $(m=$ multiplet). Infrared (IR) spectra were measured on a Jasco FT/IR-4100 Type A spectrometer with a TGS detector. Wavenumbers $\tilde{v}$ are given in $\mathrm{cm}^{-1}$ and intensities are as follows: $\mathrm{s}=$ strong, $\mathrm{m}=$ medium, $\mathrm{w}=$ weak. High-resolution mass spectra (HRMS) were recorded using an Agilent 6210 ESI-TOF or an lonspec QFT-7 ESI-TOF spectrometer. Optical rotations were measured on a JASCO P-2000 polarimeter at $589 \mathrm{~nm}$ using $100 \mathrm{~mm}$ cells and the solvent and concentration $(\mathrm{g} / 100 \mathrm{~mL})$ indicated. Melting points were measured on a Stuart SMP30. 


\section{Experimental Procedures and Characterization Data}

\subsection{Synthesis of $\mathrm{Y}$-Hydroxy Enone 6}

Ergosterol mesylate (S2)

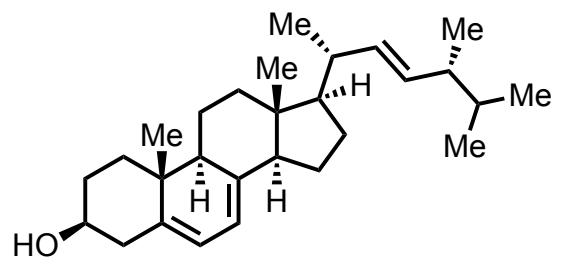

S1

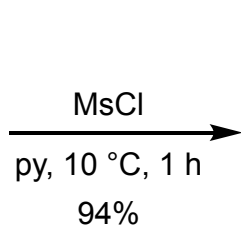

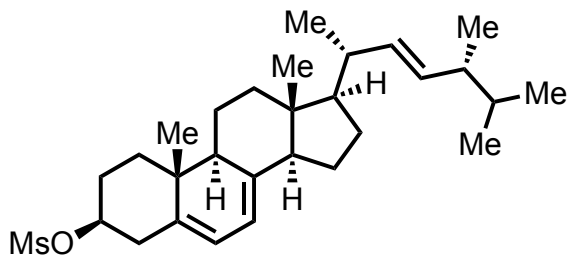

S2

To a stirred solution of ergosterol (S1) $(53.7 \mathrm{~g}, 135 \mathrm{mmol}, 1.0 \mathrm{eq}$.) in pyridine (1.0 L) was added methanesulfonyl chloride $\left(52.4 \mathrm{~mL}, 677 \mathrm{mmol}, 5.0\right.$ eq.) dropwise at $10^{\circ} \mathrm{C}$. After stirring at this temperature for $1 \mathrm{~h}$, the reaction mixture was poured into a mixture of ice and $\mathrm{H}_{2} \mathrm{O}(1.5 \mathrm{~L})$ under stirring. The precipitate was filtered off, washed with $\mathrm{H}_{2} \mathrm{O}(3 \times 1 \mathrm{~L})$, and dried under vacuum to give mesylate $\mathbf{S} 2(60.0 \mathrm{~g}, 126 \mathrm{mmol}$, $94 \%$ ) as a colorless solid, which was used in the next step without further purification.

1H-NMR: $\quad\left(500 \mathrm{MHz}, \mathrm{CDCl}_{3}\right) ; \delta[\mathrm{ppm}]=5.60(\mathrm{dd}, J=5.7,2.3 \mathrm{~Hz}, 1 \mathrm{H}), 5.39(\mathrm{dt}, J=5.3,2.6 \mathrm{~Hz}, 1 \mathrm{H})$, $5.23(\mathrm{dd}, J=15.2,7.1 \mathrm{~Hz}, 1 \mathrm{H}), 5.17(\mathrm{dd}, J=15.2,7.8 \mathrm{~Hz}, 1 \mathrm{H}), 4.63(\mathrm{tt}, J=11.4,4.7 \mathrm{~Hz}$, 1H), 3.02 (s, 3H), 2.65 (ddd, J = 14.4, 5.1, 2.4 Hz, 1H), $2.62-2-50(\mathrm{~m}, 1 \mathrm{H}), 2.15-1.21$ $(\mathrm{m}, 18 \mathrm{H}), 1.03(\mathrm{~d}, J=6.6 \mathrm{~Hz}, 3 \mathrm{H}), 0.95(\mathrm{~s}, 3 \mathrm{H}), 0.92(\mathrm{~d}, J=6.8 \mathrm{~Hz}, 3 \mathrm{H}), 0.84(\mathrm{~d}, J=6.8 \mathrm{~Hz}$, $3 \mathrm{H}), 0.82(\mathrm{~d}, J=6.9 \mathrm{~Hz}, 3 \mathrm{H}), 0.62(\mathrm{~s}, 3 \mathrm{H})$.

All characterization data were consistent with those reported in the literature.[1]

(22E)-3a,5-Cyclo-5a-ergosta-7,22-dien-6ß-ol (S3)

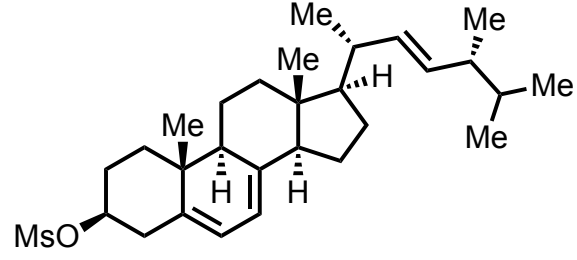

S2

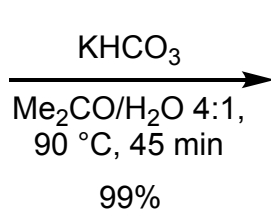

$99 \%$

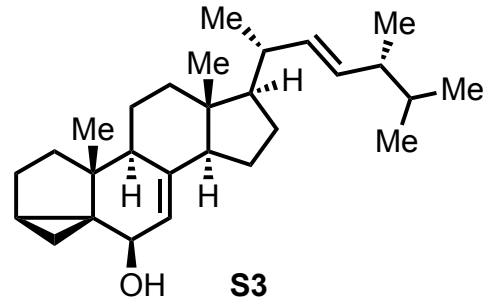

Finely powdered mesylate $S 2(60.0 \mathrm{~g}, 126 \mathrm{mmol}, 1.0 \mathrm{eq}$.) was added portion wise to a refluxing solution of $\mathrm{KHCO}_{3}(14.6 \mathrm{~g}, 145 \mathrm{mmol}, 1.15$ eq. $)$ in acetone/ $\mathrm{H}_{2} \mathrm{O}(4: 1,1.8 \mathrm{~L})$ at $80^{\circ} \mathrm{C}$. After stirring for $45 \mathrm{~min}$ at $90{ }^{\circ} \mathrm{C}$, the reaction mixture was allowed to cool to $40^{\circ} \mathrm{C}$ and a mixture of ice and $\mathrm{H}_{2} \mathrm{O}(1 \mathrm{~L})$ was added. After further cooling to $0{ }^{\circ} \mathrm{C}$, the precipitate was filtered off, washed with $\mathrm{H}_{2} \mathrm{O}(2 \times 500 \mathrm{~mL})$, and dried under vacuum to 
give $i$-sterol S3 (49.6 g, $125 \mathrm{mmol}, 99 \%)$ as a colorless solid, which was used in the next step without further purification.

1H-NMR: $\quad\left(500 \mathrm{MHz}, \mathrm{CDCl}_{3}\right) ; \delta[\mathrm{ppm}]=5.52-5.42(\mathrm{~m}, 1 \mathrm{H}), 5.23(\mathrm{dd}, J=15.2,7.3 \mathrm{~Hz}, 1 \mathrm{H}), 5.17(\mathrm{dd}$, $J=15.3,8.1 \mathrm{~Hz}, 1 \mathrm{H}), 3.42(\mathrm{~s}, 1 \mathrm{H}), 2.10-1.18(\mathrm{~m}, 20 \mathrm{H}), 1.08(\mathrm{~s}, 3 \mathrm{H}), 1.03(\mathrm{~d}, J=6.6 \mathrm{~Hz}$, $3 \mathrm{H}), 0.92(\mathrm{~d}, J=6.8 \mathrm{~Hz}, 3 \mathrm{H}), 0.84(\mathrm{~d}, J=6.8 \mathrm{~Hz}), 3 \mathrm{H}), 0.83(\mathrm{~d}, J=6.7 \mathrm{~Hz}, 3 \mathrm{H}), 0.66-0.60$ (m, 4H), 0.48 (dd, $J=7.9,5.0 \mathrm{~Hz}, 1 \mathrm{H})$.

All characterization data were consistent with those reported in the literature. ${ }^{[1]}$

(22E)-3a,5-Cyclo-5a-ergosta-7,22-dien-6-one (S4)
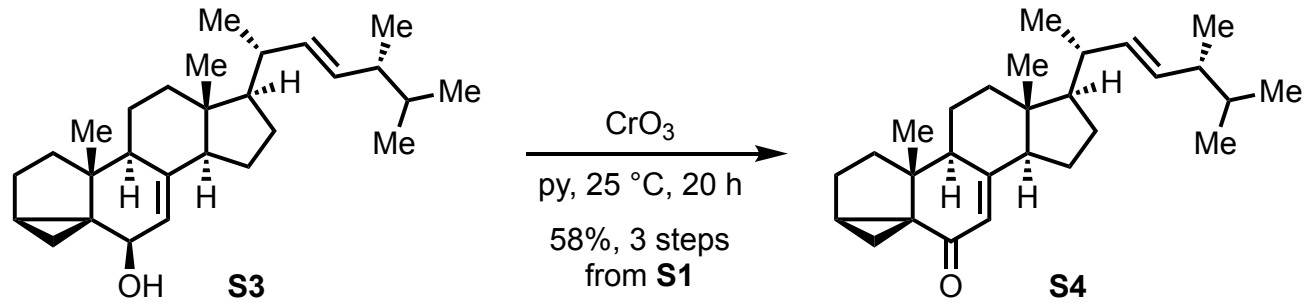

Under stirring $\mathrm{CrO}_{3}(50.0 \mathrm{~g}, 500 \mathrm{mmol}, 4.0$ eq.) was added portion wise to pyridine $(500 \mathrm{~mL})$. To the resulting red-brown suspension was added $i$-sterol $\mathbf{S} 3(49.6 \mathrm{~g}, 125 \mathrm{mmol}, 1.0$ eq.) in pyridine $(500 \mathrm{~mL})$ via cannula over $10 \mathrm{~min}$. After stirring at $25^{\circ} \mathrm{C}$ for $20 \mathrm{~h}, \mathrm{Et}_{2} \mathrm{O}(1.2 \mathrm{~L})$ was added, the resulting mixture was filtered through Celite ${ }^{\circledR}$ and rinsed with $\mathrm{Et}_{2} \mathrm{O}(2 \times 200 \mathrm{~mL})$. The filtrate was washed sequentially with $\mathrm{H}_{2} \mathrm{O}$ $(2 \times 1 \mathrm{~L})$ and brine (sat., $1 \mathrm{~L})$, dried over $\mathrm{MgSO}_{4}$, and concentrated under reduced pressure. Crystallization from acetone $(250 \mathrm{~mL})$ gave enone $\mathbf{S} 4(30.9 \mathrm{~g}, 78.3 \mathrm{mmol}, 58 \%$ over 3 steps $)$ as a crystalline solid.

1H-NMR: $\quad\left(500 \mathrm{MHz}, \mathrm{CDCl}_{3}\right) ; \delta[\mathrm{ppm}]=5.82(\mathrm{t}, J=2.2 \mathrm{~Hz}, 1 \mathrm{H}), 5.27(\mathrm{dd}, J=15.3,7.6 \mathrm{~Hz}, 1 \mathrm{H}), 5.20$ (dd, $J=15.7,8.3 \mathrm{~Hz}, 1 \mathrm{H}$ ), 2.28 (ddd, $J=11.7,7.2,2.3 \mathrm{~Hz}, 1 \mathrm{H}$ ), $2.19-2.05(\mathrm{~m}, 3 \mathrm{H}), 2.00$ (tdd, $J=12.3,8.0,4.4 \mathrm{~Hz}, 1 \mathrm{H}), 1.93-1.85(\mathrm{~m}, 1 \mathrm{H}), 184-1.64(\mathrm{~m}, 8 \mathrm{H}), 1.58-1.47(\mathrm{~m}$, 2H), $1.47-1.33(\mathrm{~m}, 3 \mathrm{H}), 1.19-1.13(\mathrm{~m}, 1 \mathrm{H}), 1.12(\mathrm{~s}, 3 \mathrm{H}), 1.07(\mathrm{~d}, J=6.6 \mathrm{~Hz}, 3 \mathrm{H}), 0.95$ (d, $J=6.9 \mathrm{~Hz}, 3 \mathrm{H}), 0.87(\mathrm{~d}, J=6.8 \mathrm{~Hz}, 3 \mathrm{H}), 0.85(\mathrm{~d}, J=6.7 \mathrm{~Hz}, 3 \mathrm{H}), 0.78(\mathrm{t}, J=4.6 \mathrm{~Hz}$, $1 \mathrm{H}), 0.71(\mathrm{~s}, 3 \mathrm{H})$.

All characterization data were consistent with those reported in the literature.[1] 
(22E)-14-Hydroxy-3a,5-cyclo-5a-ergosta-7,22-dien-6-one (6)

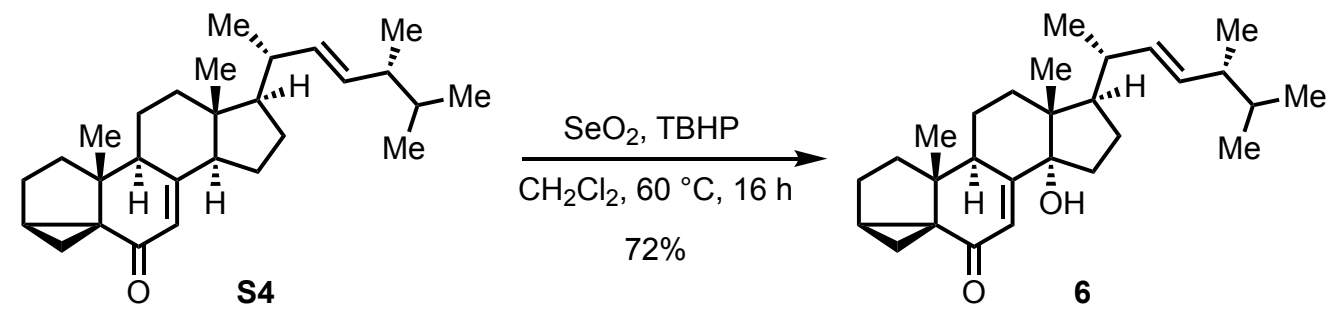

To a stirred suspension of $\mathrm{SeO}_{2}\left(1.13 \mathrm{~g}, 10.2 \mathrm{mmol}, 0.5\right.$ eq.) in $\mathrm{CH}_{2} \mathrm{Cl}_{2}(18 \mathrm{~mL})$ was added $t \mathrm{BuOOH}(70 \%$ in $\mathrm{H}_{2} \mathrm{O}, 11.2 \mathrm{~mL}, 81.8 \mathrm{mmol}, 4.0$ eq.) at $0^{\circ} \mathrm{C}$. After stirring at $25^{\circ} \mathrm{C}$ for $15 \mathrm{~min}$, enone $\mathbf{S} 4(8.07 \mathrm{~g}, 20.4 \mathrm{mmol}$, 1.0 eq.) in $\mathrm{CH}_{2} \mathrm{Cl}_{2}\left(22 \mathrm{~mL}\right.$ ) was added. The vessel was sealed, and the mixture was stirred at $60{ }^{\circ} \mathrm{C}$ for $16 \mathrm{~h}$. The reaction mixture was allowed to cool to $25^{\circ} \mathrm{C}$ and was carefully added to $\mathrm{NaHSO}_{3}\left(10 \% w / w\right.$ in $\mathrm{H}_{2} \mathrm{O}$, $200 \mathrm{~mL})$ at $0{ }^{\circ} \mathrm{C}$. The mixture was extracted with $\mathrm{CH}_{2} \mathrm{Cl}_{2}(2 \times 250 \mathrm{~mL})$ and the combined organic phases were washed sequentially with $\mathrm{NaHCO}_{3}$ (sat. aq., $250 \mathrm{~mL}$ ) and brine (sat., $250 \mathrm{~mL}$ ), dried over $\mathrm{MgSO}_{4}$ and concentrated under reduced pressure. The residue was adsorbed on silica and the mixture was purified by column chromatography (silica gel, nhexane/EtOAc 5:1 $\rightarrow$ 4:1) to give $\mathrm{Y}$-hydroxy enone 6 (6.05 g, $14.7 \mathrm{mmol}, 72 \%$ ) as a crystalline solid.

1H-NMR: $\quad\left(700 \mathrm{MHz}, \mathrm{CDCl}_{3}\right) ; \delta[p p m]=5.97(\mathrm{~d}, J=2.7 \mathrm{~Hz}, 1 \mathrm{H}), 5.27(\mathrm{dd}, J=15.2,7.6 \mathrm{~Hz}, 1 \mathrm{H}), 5.20$ (ddd, $J=15.2,8.5,0.9 \mathrm{~Hz}, 1 \mathrm{H}), 2.79-2.75(\mathrm{~m}, 1 \mathrm{H}), 2.13-1.84(\mathrm{~m}, 7 \mathrm{H}), 1.80$ (dt, $J=8.8$, $4.6 \mathrm{~Hz}, 1 \mathrm{H}), 1.75-1.65(\mathrm{~m}, 6 \mathrm{H}), 1.55$ (ddd, $J=13.7,9.8,5.1 \mathrm{~Hz}, 1 \mathrm{H}), 1.48$ (qd, $J=6.8$, $5.7 \mathrm{~Hz}, 1 \mathrm{H}), 1.43(\mathrm{~s}, 1 \mathrm{H}), 1.43-1.37(\mathrm{~m}, 1 \mathrm{H}), 1.15-1.11(\mathrm{~m}, 1 \mathrm{H}), 1.10(\mathrm{~s}, 3 \mathrm{H}), 1.03(\mathrm{~d}, J$ $=6.5 \mathrm{~Hz}, 3 \mathrm{H}), 0.92(\mathrm{~d}, J=6.8 \mathrm{~Hz}, 3 \mathrm{H}), 0.84(\mathrm{~d}, J=6.8 \mathrm{~Hz}, 3 \mathrm{H}), 0.83(\mathrm{~d}, J=6.8 \mathrm{~Hz}, 3 \mathrm{H})$, $0.79(\mathrm{t}, J=4.7 \mathrm{~Hz}, 1 \mathrm{H}), 0.76(\mathrm{~s}, 3 \mathrm{H})$.

All characterization data were consistent with those reported in the literature.[2] 


\subsection{Studies on the Radical Rearrangement}

Reaction Conditions for Entry 1, Table 1

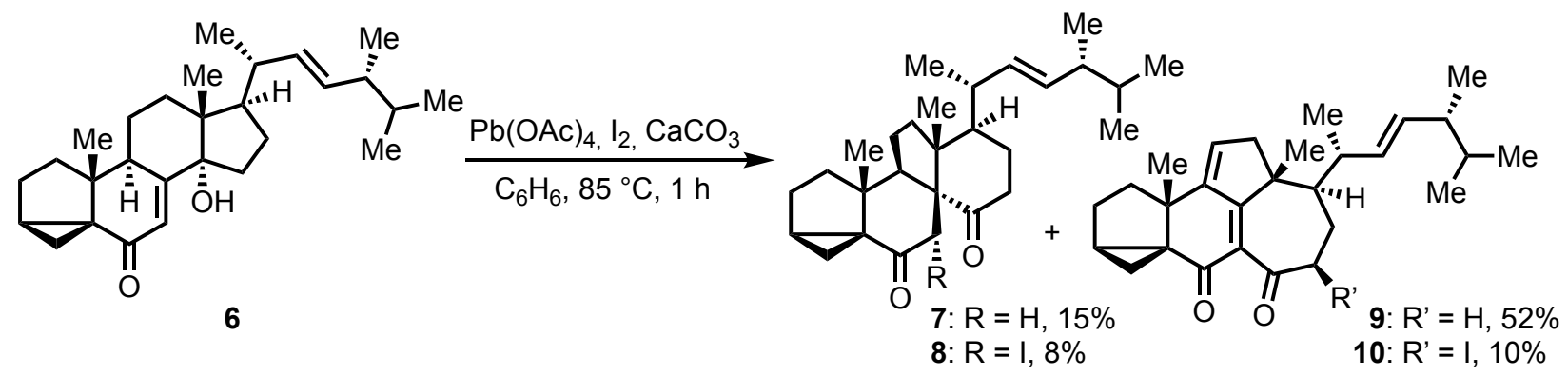

Through a solution of $\mathrm{Y}$-hydroxy enone $6(1.00 \mathrm{~g}, 2.44 \mathrm{mmol}, 1.0 \mathrm{eq}$.) in benzene $(100 \mathrm{~mL})$ was bubbled argon via cannula for $10 \mathrm{~min} . \mathrm{CaCO}_{3}(487 \mathrm{mg}, 4.87 \mathrm{mmol}, 2.0$ eq.), iodine (1.24 g, $4.87 \mathrm{mmol}, 2.0$ eq.), and $\mathrm{Pb}(\mathrm{OAc})_{4}\left(2.16 \mathrm{~g}, 4.87 \mathrm{mmol}, 2.0\right.$ eq.) were added and the reaction mixture was stirred at $85^{\circ} \mathrm{C}$ for $1 \mathrm{~h}$. It was cooled to $25^{\circ} \mathrm{C}$, filtered through a plug of Celite ${ }^{\circledR}$, and rinsed with EtOAc $(50 \mathrm{~mL})$. The organic phase was washed sequentially with $\mathrm{Na}_{2} \mathrm{~S}_{2} \mathrm{O}_{3}$ (sat. aq., $150 \mathrm{~mL}$ ) and brine (sat., $150 \mathrm{~mL}$ ), dried over $\mathrm{MgSO}_{4}$, and the solvent was removed under reduced pressure. Column chromatography (silica gel, nhexane/EtOAc 9:1 $\rightarrow 3: 1$ ) gave dione 7 (147 mg, $358 \mu \mathrm{mol}, 15 \%$ ) as a colorless solid, iodo dione 8 (107 mg, $199 \mu \mathrm{mol}, 8 \%$ ) as colorless needles, diene dione 9 (517 mg, $1.27 \mathrm{mmol}, 52 \%$ ) as a light-yellow foam, and iodo diene dione 10 (130 mg, $244 \mu \mathrm{mol}, 10 \%$ ) as a colorless solid.

Characterization data for (22E)-13(14 $\rightarrow 8)$ abeo-3a,5-Cyclo-5a-ergosta-22-en-6,14-dione (7)

M.p.: $\quad 66-67^{\circ} \mathrm{C}\left(\mathrm{CHCl}_{3}\right)$.

TLC: $\quad R_{\mathrm{f}}=0.39($ nhexane/EtOAc $9: 1)$.

1H-NMR: $\quad\left(700 \mathrm{MHz}, \mathrm{CDCl}_{3}\right) ; \delta[\mathrm{ppm}]=5.27-5.24(\mathrm{~m}, 2 \mathrm{H}), 2.93(\mathrm{dd}, J=11.0,8.2 \mathrm{~Hz}, 1 \mathrm{H}), 2.73(\mathrm{~d}$, $J=14.5 \mathrm{~Hz}, 1 \mathrm{H}), 2.61$ (ddd, $J=17.1,6.0,3.2 \mathrm{~Hz}, 1 \mathrm{H}), 2.52(\mathrm{~d}, J=14.6 \mathrm{~Hz}, 1 \mathrm{H}), 2.38-$ $2.31(\mathrm{~m}, 1 \mathrm{H}), 2.28-2.18(\mathrm{~m}, 2 \mathrm{H}), 1.91-1.82(\mathrm{~m}, 2 \mathrm{H}), 1.82-1.75(\mathrm{~m}, 3 \mathrm{H}), 1.75-1.70$ $(\mathrm{m}, 1 \mathrm{H}), 1.67-1.61(\mathrm{~m}, 3 \mathrm{H}), 1.51(\mathrm{dd}, J=8.3,4.3 \mathrm{~Hz}, 1 \mathrm{H}), 1.50-1.44(\mathrm{~m}, 1 \mathrm{H}), 1.31$ (td, $J=12.0,6.3 \mathrm{~Hz}, 1 \mathrm{H}), 1.20-1.12(\mathrm{~m}, 1 \mathrm{H}), 1.10(\mathrm{~s}, 3 \mathrm{H}), 1.09-1.03(\mathrm{~m}, 1 \mathrm{H}), 1.03(\mathrm{~s}, 3 \mathrm{H})$, $1.02(\mathrm{~d}, J=7.0 \mathrm{~Hz}, 3 \mathrm{H}), 0.93(\mathrm{~d}, J=6.8 \mathrm{~Hz}, 3 \mathrm{H}), 0.84(\mathrm{~d}, J=6.8 \mathrm{~Hz}, 3 \mathrm{H}), 0.84-0.82(\mathrm{~m}$, $1 \mathrm{H}), 0.82(\mathrm{~d}, J=6.8 \mathrm{~Hz}, 3 \mathrm{H})$.

${ }^{13} \mathrm{C}-\mathrm{NMR}: \quad\left(176 \mathrm{MHz}, \mathrm{CDCl}_{3}\right) ; \delta[\mathrm{ppm}]=215.5,210.6,134.7,133.1,61.4,52.9,52.5,45.2,44.9,44.2$, 43.4, 41.9, 41.3, 38.9, 38.3, 38.1, 37.9, 33.3, 25.6, 25.2, 23.2, 22.3, 21.7, 20.2, 19.8, 17.8, $17.5(2 \mathrm{C})$. 
IR: $\quad\left(\right.$ neat); $\tilde{v}\left[\mathrm{~cm}^{-1}\right]=2953(\mathrm{~m}), 2922(\mathrm{~s}), 2853(\mathrm{~m}), 1697(\mathrm{w}), 1461(\mathrm{w}), 1376(\mathrm{w}), 1158(\mathrm{w})$, $972(\mathrm{w})$.

HRMS: $\quad$ (ESI-TOF) $m / z$ calcd. for $\mathrm{C}_{28} \mathrm{H}_{42} \mathrm{O}_{2} \mathrm{Na}^{+}[\mathrm{M}+\mathrm{Na}]^{+}:$433.3077, found: 433.3088 .

Opt. act. $[\mathrm{a}]_{\mathrm{D}}^{21}=+34.0\left(c=1.00, \mathrm{CHCl}_{3}\right)$.

Characterization data for (22E)-7a-lodo-13(14 $\rightarrow 8)$ abeo-3a,5-Cyclo-5a-ergosta-22-en-6,14-dione (8)

$\begin{array}{ll}\text { M.p.: } & 102-103{ }^{\circ} \mathrm{C}(\mathrm{EtOAc}) . \\ \text { TLC: } & R_{\mathrm{f}}=0.27(\text { nhexane/EtOAc 9:1). } \\ \text { 1H-NMR: } & \left(700 \mathrm{MHz}, \mathrm{CDCl}_{3}\right) ; \delta[\mathrm{ppm}]=5.40(\mathrm{dd}, J=15.3,7.8 \mathrm{~Hz}, 1 \mathrm{H}), 5.29(\mathrm{dd}, J=15.4,8.2 \mathrm{~Hz}, \\ & 1 \mathrm{H}), 5.13(\mathrm{~s}, 1 \mathrm{H}), 2.73(\mathrm{dd}, J=19.2,4.9 \mathrm{~Hz}, 1 \mathrm{H}), 2.56(\mathrm{t}, J=7.3 \mathrm{~Hz}, 1 \mathrm{H}), 2.53(\mathrm{~d}, J=9.3 \\ & \mathrm{Hz}, 1 \mathrm{H}), 2.24(\mathrm{dt}, J=8.3,5.2 \mathrm{~Hz}, 1 \mathrm{H}), 2.16-2.08(\mathrm{~m}, 1 \mathrm{H}), 2.07-2.03(\mathrm{~m}, 1 \mathrm{H}), 2.00(\mathrm{td}, \\ & J=12.9,5.1 \mathrm{~Hz}, 1 \mathrm{H}), 1.93-1.85(\mathrm{~m}, 2 \mathrm{H}), 1.78-1.71(\mathrm{~m}, 2 \mathrm{H}), 1.69(\mathrm{dd}, J=8.4,4.2 \mathrm{~Hz}, \\ & 1 \mathrm{H}), 1.65(\mathrm{dd}, J=13.2,7.6 \mathrm{~Hz}, 1 \mathrm{H}), 1.63-1.57(\mathrm{~m}, 2 \mathrm{H}), 1.53-1.47(\mathrm{~m}, 2 \mathrm{H}), 1.42(\mathrm{~s}, 3 \mathrm{H}), \\ & 1.35(\mathrm{~d}, J=12.4 \mathrm{~Hz}, 1 \mathrm{H}), 1.19-1.14(\mathrm{~m}, 1 \mathrm{H}), 1.13(\mathrm{~s}, 3 \mathrm{H}), 1.09(\mathrm{~d}, J=7.1 \mathrm{~Hz}, 3 \mathrm{H}), 0.97 \\ & -0.95(\mathrm{~m}, 1 \mathrm{H}), 0.95(\mathrm{~d}, J=6.8 \mathrm{~Hz}, 3 \mathrm{H}), 0.86(\mathrm{~d}, J=6.7 \mathrm{~Hz}, 3 \mathrm{H}), 0.84(\mathrm{~d}, J=6.8 \mathrm{~Hz}, 3 \mathrm{H}) . \\ & (176 \mathrm{MHz}, \mathrm{CDCl}) ; \delta[\mathrm{ppm}]=216.2,200.0,135.0,132.5,69.3,55.4,51.7,48.5,48.4,44.8, \\ & 43.5,42.3,41.3,41.1,39.1,37.9,37.5,33.3,24.6,24.5,23.9,23.0,21.8,20.2,19.9,19.7, \\ & 19.0,17.8 . \\ & (\mathrm{neat}) ; \tilde{v}\left[\mathrm{~cm}^{-1}\right]=2955(\mathrm{~s}), 2871(\mathrm{~m}), 2360(\mathrm{~s}), 2341(\mathrm{~m}), 1699(\mathrm{~s}), 1456(\mathrm{~m}), 1364(\mathrm{w}), \\ & 1288(\mathrm{w}), 1162(\mathrm{w}), 1082(\mathrm{w}), 983(\mathrm{~m}) .\end{array}$

HRMS: $\quad$ (ESI-TOF) $\mathrm{m} / \mathrm{z}$ calcd. for $\mathrm{C}_{28} \mathrm{H}_{41} \mathrm{IO}_{2} \mathrm{Na}^{+}[\mathrm{M}+\mathrm{Na}]+:$ 559.2043, found: 559.2055.

Opt. act. $[\mathrm{a}]_{\mathrm{D}}^{21}=+41.6\left(c=1.01, \mathrm{CHCl}_{3}\right)$.

Characterization data for $(22 E)-13(14 \rightarrow 8), 14(8 \rightarrow 7)$ diabeo-3a,5-cyclo-5a-ergosta-7,9(11),22-trien-6,14dione (9)

TLC: $\quad R_{\mathrm{f}}=0.32($ nhexane/EtOAc 3:1),

1H-NMR: $\quad\left(500 \mathrm{MHz}, \mathrm{CDCl}_{3}\right) ; \delta[\mathrm{ppm}]=6.34(\mathrm{t}, J=2.9 \mathrm{~Hz}, 1 \mathrm{H}), 5.36(\mathrm{dd}, J=15.3,8.4 \mathrm{~Hz}, 1 \mathrm{H}), 5.28$ (dd, $J=15.3,7.9 \mathrm{~Hz}, 1 \mathrm{H}), 2.78(\mathrm{dd}, J=17.7,2.4 \mathrm{~Hz}, 1 \mathrm{H}), 2.74-2.68(\mathrm{~m}, 1 \mathrm{H}), 2.62-2.54$ (m, 2H), 2.45 (dd, $J=17.6,3.4 \mathrm{~Hz}, 1 \mathrm{H}), 2.11-2.03(\mathrm{~m}, 1 \mathrm{H}), 2.03-1.95(\mathrm{~m}, 1 \mathrm{H}), 1.93-$ $1.81(\mathrm{~m}, 4 \mathrm{H}), 1.77-1.72(\mathrm{~m}, 2 \mathrm{H}), 1.69(\mathrm{dd}, J=12.9,7.6 \mathrm{~Hz}, 1 \mathrm{H}), 1.50-1.37(\mathrm{~m}, 2 \mathrm{H})$, $1.16(\mathrm{~s}, 6 \mathrm{H}), 1.08$ (d, $J=6.9 \mathrm{~Hz}, 3 \mathrm{H}), 0.91$ (d, $J=6.9 \mathrm{~Hz}, 3 \mathrm{H}), 0.83(\mathrm{~d}, J=7.1 \mathrm{~Hz}, 3 \mathrm{H})$, $0.81(\mathrm{~d}, J=7.0 \mathrm{~Hz}, 3 \mathrm{H}), 0.77(\mathrm{t}, J=7.1 \mathrm{~Hz}, 1 \mathrm{H})$. 
${ }^{13} \mathrm{C}-\mathrm{NMR}: \quad\left(126 \mathrm{MHz}, \mathrm{CDCl}_{3}\right) ; \delta[\mathrm{ppm}]=204.3,193.1,168.6,145.9,136.9,135.4,132.0,130.9,53.4$, $49.9,47.7,45.3,43.5,43.4,41.2,39.7,36.3,35.4,33.2,26.4,26.3,23.9,23.0,22.2,20.2$, 19.9, 17.6, 12.8 .

IR: $\quad\left(\right.$ neat); $\tilde{v}\left[\mathrm{~cm}^{-1}\right]=2957(\mathrm{~m}), 2867(\mathrm{w}), 1691(\mathrm{~m}), 1636(\mathrm{~m}), 1588(\mathrm{w}), 1454(\mathrm{w}), 1373(\mathrm{~m})$, $1260(\mathrm{~m}), 1010$ (s), $796(\mathrm{~s})$.

HRMS: (ESI-TOF); $m / z$ calcd. for $\mathrm{C}_{28} \mathrm{H}_{38} \mathrm{O}_{2} \mathrm{Na}^{+}[\mathrm{M}+\mathrm{Na}]^{+}:$429.2764, found: 429.2777.

Opt. act. $[\mathrm{a}]_{\mathrm{D}}^{23}=-13.6\left(c=1.00, \mathrm{CHCl}_{3}\right)$.

Characterization data for $(22 E)-15 \beta$-lodo-13(14 $\rightarrow 8), 14(8 \rightarrow 7)$ diabeo-3a,5-cyclo-5a-ergosta-7,9(11),22trien-6,14-dione (10)

M.p.: $\quad 155-157^{\circ} \mathrm{C}\left(\mathrm{Et}_{2} \mathrm{O}\right)$.

TLC: $\quad R_{\mathrm{f}}=0.26($ hhexane/EtOAc 5:1).

1H-NMR: $\quad\left(700 \mathrm{MHz}, \mathrm{CDCl}_{3}\right) ; \delta[\mathrm{ppm}]=6.39(\mathrm{dd}, J=3.3,2.5 \mathrm{~Hz}, 1 \mathrm{H}), 5.32-5.28(\mathrm{~m}, 2 \mathrm{H}), 4.91$ (dd, $J=4.5,3.4 \mathrm{~Hz}, 1 \mathrm{H}), 2.91(\mathrm{dd}, J=17.8,2.5 \mathrm{~Hz}, 1 \mathrm{H}), 2.70-2.65(\mathrm{~m}, 1 \mathrm{H}), 2.48(\mathrm{dd}, J=$ 17.8, 3.3 Hz, 1H), $2.21-2.18(\mathrm{~m}, 1 \mathrm{H}), 2.10-2.02(\mathrm{~m}, 3 \mathrm{H}), 1.94-1.87(\mathrm{~m}, 3 \mathrm{H}), 1.82-$ $1.75(\mathrm{~m}, 2 \mathrm{H}), 1.72(\mathrm{dd}, J=12.9,7.5 \mathrm{~Hz}, 1 \mathrm{H}), 1.50-1.44(\mathrm{~m}, 1 \mathrm{H}), 1.17(\mathrm{~s}, 3 \mathrm{H}), 1.14(\mathrm{~s}$, 3H), 1.09 (d, $J=6.9 \mathrm{~Hz}, 3 \mathrm{H}), 0.92(\mathrm{~d}, J=6.8 \mathrm{~Hz}, 3 \mathrm{H}), 0.85-0.81(\mathrm{~m}, 7 \mathrm{H})$.

${ }^{13}$ C-NMR: $\quad\left(176 \mathrm{MHz}, \mathrm{CDCl}_{3}\right) ; \delta[\mathrm{ppm}]=195.6,192.9,167.1,145.7,138.1,136.0,132.0,128.2,52.6$, 47.2 , 45.5, 43.4 (2C), 40.7, 39.4, 39.0, 36.0, 35.6, 33.2, 31.5, 26.5, 26.3, 22.8, 22.1, 20.2, 19.9, 17.6, 12.4.

FT-IR: $\quad\left(\right.$ neat); $\tilde{v}\left[\mathrm{~cm}^{-1}\right]=2955(\mathrm{~m}), 2867(\mathrm{w}), 1685(\mathrm{~s}), 1640(\mathrm{~m}), 1586(\mathrm{~m}), 1452(\mathrm{~m}), 1373(\mathrm{~m})$, $1294(\mathrm{~m}), 1136(\mathrm{~m}), 1011(\mathrm{~s}), 988(\mathrm{~m}), 880(\mathrm{~m}), 806(\mathrm{~m})$.

HRMS: (ESI-TOF); $m / z$ calcd. for $\mathrm{C}_{28} \mathrm{H}_{37} \mathrm{IO}_{2} \mathrm{Na}^{+}[\mathrm{M}+\mathrm{Na}]+:$ 555.1730, found: 555.1738.

Opt. act. $[a]_{D}^{23}=+42.0\left(c=0.41, \mathrm{CHCl}_{3}\right)$.

Reaction Conditions for Entry 2, Table 1
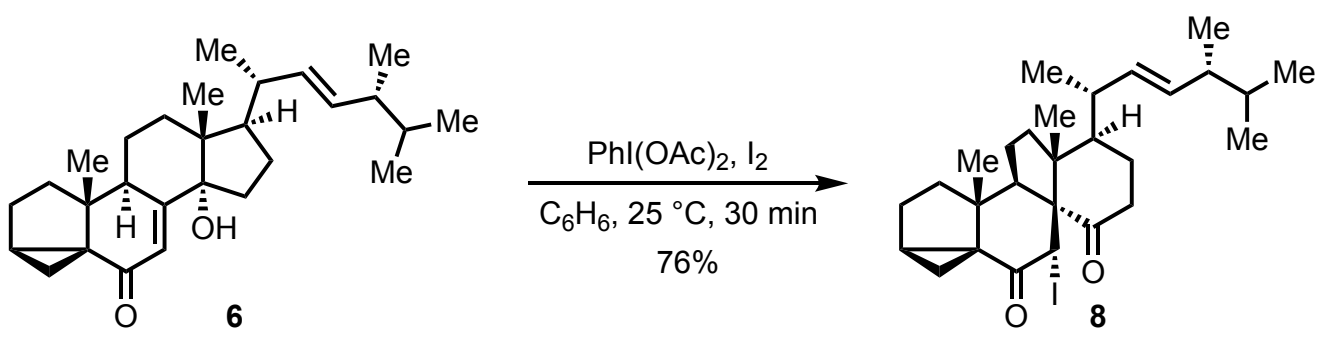
A solution of $\mathrm{Y}$-hydroxy enone 6 (30 mg, $73 \mu \mathrm{mol}, 1.0$ eq.) in benzene (1.5 mL) was degassed applying three freeze-pump-thaw cycles. (Diacetoxyiodo)benzene ( $47.0 \mathrm{mg}, 146 \mu \mathrm{mol}, 2.0$ eq.) and iodine (18 mg, $73 \mu \mathrm{mol}, 1.0$ eq.) were added, and the resulting mixture was stirred at $25^{\circ} \mathrm{C}$ for 30 min. $\mathrm{Na}_{2} \mathrm{~S}_{2} \mathrm{O}_{3}$ (sat. aq., $5 \mathrm{~mL}$ ) was added and the aqueous phase was extracted with EtOAc $(3 \times 5 \mathrm{~mL})$. The combined organic phases were washed with brine (sat., $15 \mathrm{~mL}$ ) and dried over $\mathrm{MgSO}_{4}$. The solvent was removed under reduced pressure and column chromatography (silica gel, nhexane/EtOAc 19:1 $\rightarrow$ 9:1) gave iodo ketone 8 (30 mg, $55 \mathrm{mmol}, 76 \%$ ) as colorless needles.

Characterization data for iodo ketone 8 see page S7.

Reaction Conditions for Entry 3, Table 1

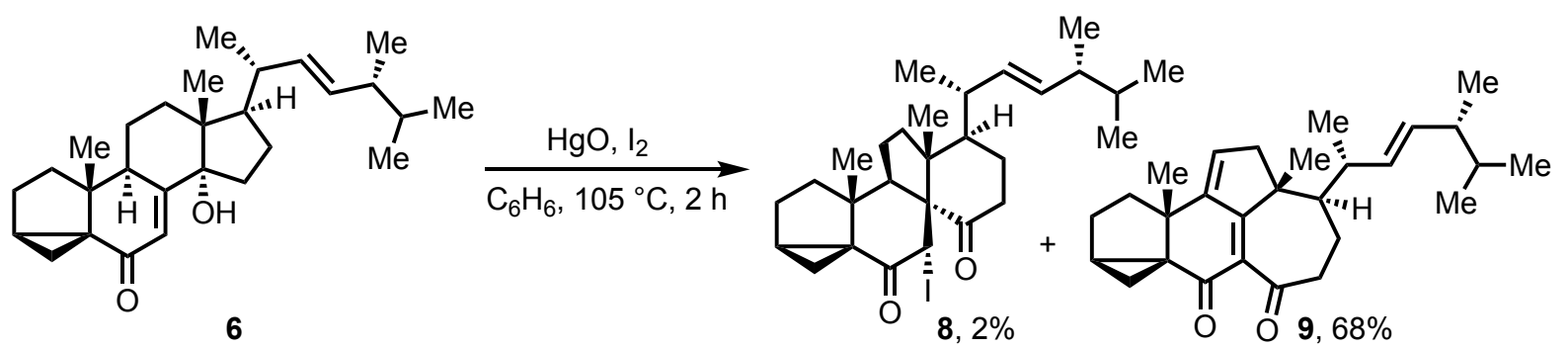

Through a solution of $\mathrm{Y}$-hydroxy enone $6(3.00 \mathrm{~g}, 7.31 \mathrm{mmol}, 1.0 \mathrm{eq}$.) in benzene ( $300 \mathrm{~mL})$ was bubbled argon via cannula for $10 \mathrm{~min}$. lodine $(4.45 \mathrm{~g}, 17.5 \mathrm{mmol}, 2.4$ eq.) and $\mathrm{HgO}$ (yellow, $4.27 \mathrm{~g}, 19.7 \mathrm{mmol}$, 2.7 eq.) were added at $60^{\circ} \mathrm{C}$ and the resulting mixture was stirred at $105^{\circ} \mathrm{C}$ for $3 \mathrm{~h}$. The reaction mixture was cooled to $25^{\circ} \mathrm{C}$, filtered through a plug of Celite ${ }^{\circledR}$, and rinsed with EtOAc $(100 \mathrm{~mL})$. The organic phase was washed sequentially with $\mathrm{Na}_{2} \mathrm{~S}_{2} \mathrm{O}_{3}$ (sat. aq., $250 \mathrm{~mL}$ ) and brine (sat., $250 \mathrm{~mL}$ ), dried over $\mathrm{MgSO}_{4}$, and the solvent was removed under reduced pressure. Column chromatography (silica gel, nhexane/EtOAc 5:1 $\rightarrow$ 3:1) gave iodo ketone 8 (78 mg, $146 \mu \mathrm{mol}, 2 \%)$ as colorless needles and diene dione 9 (2.02 g, $4.97 \mathrm{mmol}, 68 \%$ ) as a light-yellow foam.

Characterization data for iodo ketone 8 and diene dione 9 see page S7-8. 


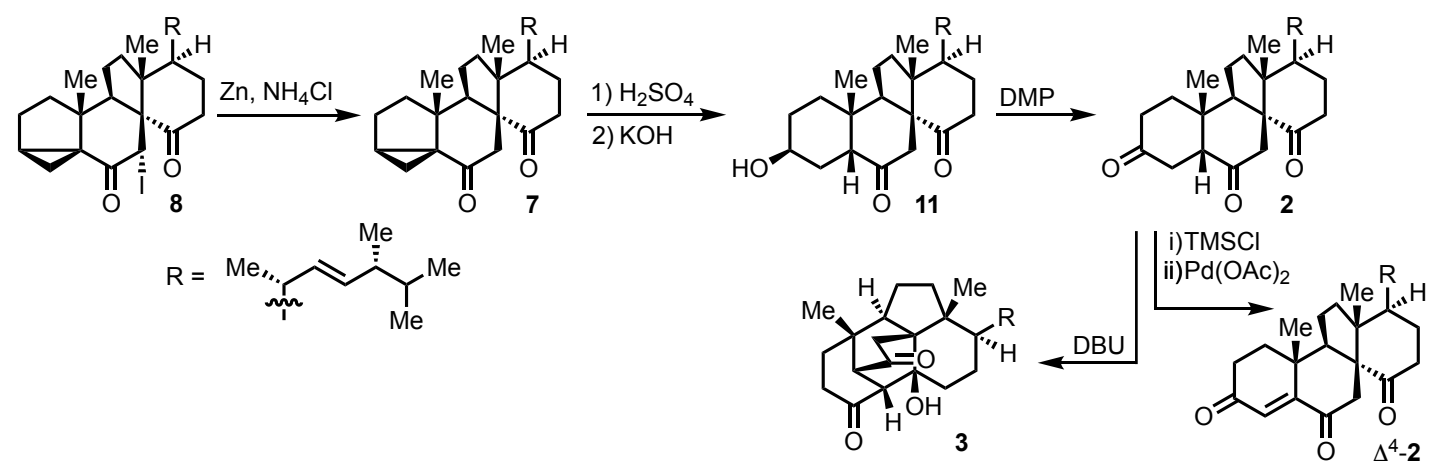

$(22 E)-13(14 \rightarrow 8)$ abeo-3a,5-Cyclo-5a-ergosta-22-en-6,14-dione (7)

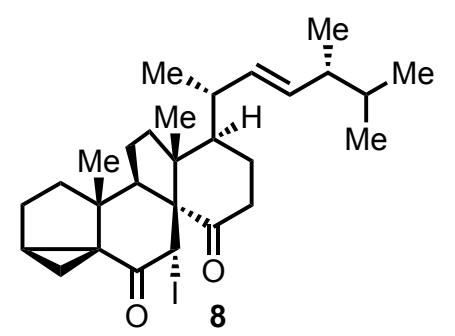

$\underset{\mathrm{MeOH}, 25^{\circ} \mathrm{C}, 16 \mathrm{~h}}{\longrightarrow}$ $91 \%$

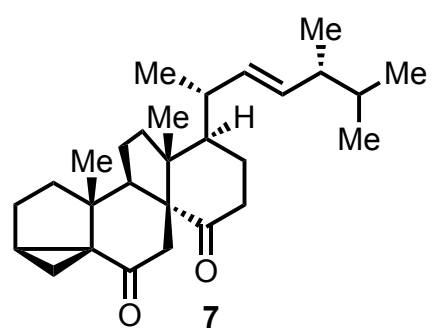

To a solution of iodo ketone 8 (107 mg, $199 \mu$ mol, 1.0 eq.) in $\mathrm{MeOH} \mathrm{(4} \mathrm{mL)} \mathrm{were} \mathrm{added} \mathrm{NH}_{4} \mathrm{Cl}(50.0 \mathrm{mg}$, $935 \mu \mathrm{mol}, 4.7$ eq.) and zink powder (208 mg, $3.18 \mathrm{mmol}, 16 \mathrm{eq}$.), and the resulting mixture was stirred at $25{ }^{\circ} \mathrm{C}$ for $16 \mathrm{~h}$. The reaction mixture was filtered through a plug of Celite ${ }^{\circledR}$, rinsed with EtOAc $(10 \mathrm{~mL})$, and $\mathrm{H}_{2} \mathrm{O}(10 \mathrm{~mL})$ was added to the resulting filtrate. The aqueous phase was extracted with EtOAc $(2 \times 10 \mathrm{~mL})$ and the combined organic phases were washed with brine (sat., $30 \mathrm{~mL}$ ), dried over $\mathrm{MgSO}_{4}$, and the solvent was removed under reduced pressure. Column chromatography (silica gel, nhexane/EtOAc 9:1) gave dione 7 (74 mg, $181 \mu \mathrm{mol}, 91 \%)$ as a colorless solid.

Characterization data for dione 7 see page S6-7. 

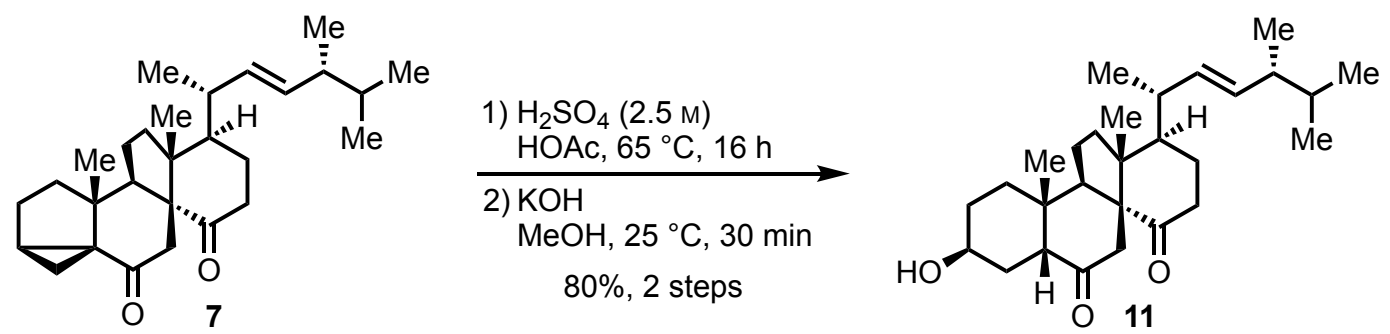

To a solution of dione 7 (133 mg, $327 \mu$ mol, 1.0 eq.) in acetic acid ( $26 \mathrm{~mL}$ ) was added $\mathrm{H}_{2} \mathrm{SO}_{4}\left(2.5 \mathrm{M} \mathrm{in} \mathrm{H}_{2} \mathrm{O}\right.$, $6.5 \mathrm{~mL}, 16 \mathrm{mmol}, 49$ eq.) and the resulting solution was stirred at $65^{\circ} \mathrm{C}$ for $16 \mathrm{~h}$. After cooling to $25^{\circ} \mathrm{C}$, the reaction mixture was poured into $\mathrm{H}_{2} \mathrm{O}(50 \mathrm{~mL})$, before $\mathrm{NaOH}\left(20 \% w / w\right.$ in $\left.\mathrm{H}_{2} \mathrm{O}, 8.7 \mathrm{~mL}\right)$ was carefully added. The aqueous phase was extracted with $\mathrm{Et}_{2} \mathrm{O}(3 \times 25 \mathrm{~mL})$ and the combined organic phases were washed with brine (sat., $75 \mathrm{~mL}$ ), dried over $\mathrm{MgSO}_{4}$, and the solvent was removed under reduced pressure. The crude product was then dissolved in $\mathrm{KOH}(5 \%$ w/w in $\mathrm{MeOH}, 5 \mathrm{~mL})$ and the reaction mixture was stirred at $25^{\circ} \mathrm{C}$ for 30 min. After diluting with $\mathrm{H}_{2} \mathrm{O}(5 \mathrm{~mL})$, it was neutralized with $\mathrm{HCl}\left(0.5 \mathrm{M} \mathrm{in} \mathrm{H}_{2} \mathrm{O}\right)$, and the aqueous phase was extracted with EtOAc $(3 \times 10 \mathrm{~mL})$. The combined organic phases were washed with brine (sat., $30 \mathrm{~mL}$ ), dried over $\mathrm{MgSO}_{4}$, and the solvent was removed under reduced pressure. Column chromatography (silica gel, nhexane/EtOAc 1:1) gave 3ß-hydroxy dione 11 (112 mg, $262 \mu \mathrm{mol}, 80 \%$ over 2 steps) as a colorless solid.

M.p.: $\quad 167-168^{\circ} \mathrm{C}\left(\mathrm{CHCl}_{3}\right)$.

TLC: $\quad R_{\mathrm{f}}=0.36($ nhexane/EtOAc 1:2).

1H-NMR: $\quad\left(700 \mathrm{MHz}, \mathrm{CDCl}_{3}\right) ; \delta[\mathrm{ppm}]=5.26-5.19(\mathrm{~m}, 2 \mathrm{H}), 3.79-3.72(\mathrm{~m}, 1 \mathrm{H}), 2.94-2.90(\mathrm{~m}$, $1 \mathrm{H}), 2.83(\mathrm{dd}, J=14.0,2.0 \mathrm{~Hz}, 1 \mathrm{H}), 2.81-2.75(\mathrm{~m}, 1 \mathrm{H}), 2.46-2.43(\mathrm{~m}, 1 \mathrm{H}), 2.43-2.37$ (m, 1H), $2.37-2.31(\mathrm{~m}, 2 \mathrm{H}), 2.25-2.17(\mathrm{~m}, 1 \mathrm{H}), 2.08$ (ddd, J = 14.0, 9.9, 4.2 Hz, 1H), $2.03-1.99(\mathrm{~m}, 1 \mathrm{H}), 1.98-1.93(\mathrm{~m}, 2 \mathrm{H}), 1.89(\mathrm{~d}, J=13.8 \mathrm{~Hz}, 1 \mathrm{H}), 1.85-1.81(\mathrm{~m}, 1 \mathrm{H})$, $1.67-1.58(\mathrm{~m}, 3 \mathrm{H}), 1.55$ (ddd, $J=14.0,12.3,6.0 \mathrm{~Hz}, 1 \mathrm{H}), 1.48-1.41(\mathrm{~m}, 2 \mathrm{H}), 1.33$ (ddd, $J=12.8,11.3,4.8 \mathrm{~Hz}, 1 \mathrm{H}), 1.20(\mathrm{td}, J=13.3,4.0 \mathrm{~Hz}, 1 \mathrm{H}), 1.14-1.11(\mathrm{~m}, 6 \mathrm{H}), 1.09$ (dq, $J=13.3,3.7 \mathrm{~Hz}, 1 \mathrm{H}), 0.87(\mathrm{~d}, J=6.8 \mathrm{~Hz}, 3 \mathrm{H}), 0.80$ (d, $J=6.8 \mathrm{~Hz}, 3 \mathrm{H}), 0.79(\mathrm{~d}, J=6.8 \mathrm{~Hz}$, $3 \mathrm{H}), 0.74(\mathrm{~s}, 3 \mathrm{H})$.

13C-NMR: $\quad\left(176 \mathrm{MHz}, \mathrm{CDCl}_{3}\right) ; \delta[\mathrm{ppm}]=215.9,209.4,135.2,132.3,66.3,65.0,59.7,53.7,49.5,45.8$, $43.4,40.3,40.0,38.8,36.9,34.1,33.7,33.2,30.4,29.4,27.3,25.7,24.2,24.1,20.2,19.8$, 17.7, 15.5 .

IR: $\quad$ (neat); $\tilde{v}\left[\mathrm{~cm}^{-1}\right]=3412(\mathrm{br} \mathrm{w}), 2954(\mathrm{~m}), 2924(\mathrm{~s}), 2854(\mathrm{~m}), 1709(\mathrm{~m}), 1459(\mathrm{w}), 1379(\mathrm{w})$, $1161(w), 1059(w), 976(w)$.

HRMS: $\quad$ (ESI-TOF); $m / z$ calcd. for $\mathrm{C}_{28} \mathrm{H}_{44} \mathrm{O}_{3} \mathrm{Na}^{+}[\mathrm{M}+\mathrm{Na}]^{+}: 451.3183$, found: 451.3198. 
Opt. act. $\quad[a]_{D}^{21}=+6.1\left(c=1.00, \mathrm{CHCl}_{3}\right)$.

Dankasterone B (2)
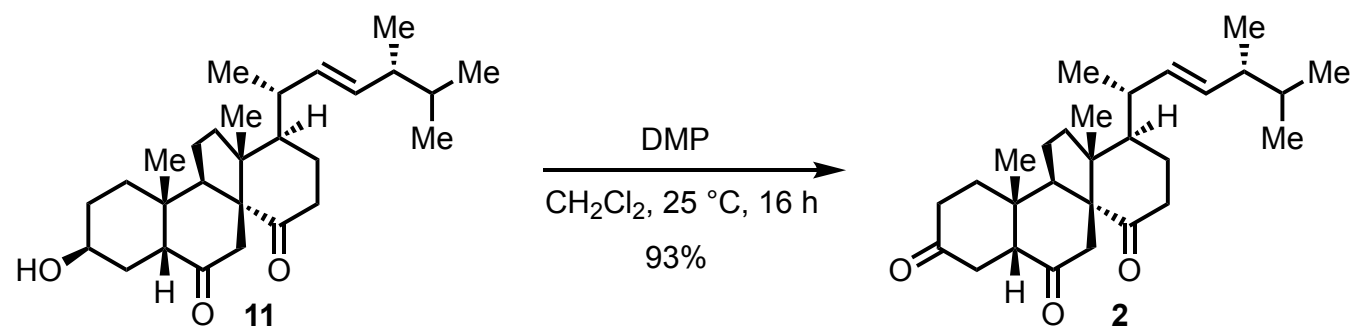

To a solution of $3 \beta$-hydroxy dione 11 (15 mg, $35 \mu \mathrm{mol}, 1.0$ eq.) in $\mathrm{CH}_{2} \mathrm{Cl}_{2}$ (1.5 mL) was added Dess-Martin periodinane (22 mg, $53 \mu \mathrm{mol}, 1.5 \mathrm{eq}$.) and the reaction mixture was stirred at $25^{\circ} \mathrm{C}$ for $16 \mathrm{~h}$. The reaction mixture was then diluted with $\mathrm{CH}_{2} \mathrm{Cl}_{2}\left(2 \mathrm{~mL}\right.$ ) and $\mathrm{Na}_{2} \mathrm{~S}_{2} \mathrm{O}_{3}$ (sat. aq., $5 \mathrm{~mL}$ ) was added. The aqueous phase was extracted with $\mathrm{CH}_{2} \mathrm{Cl}_{2}(3 \times 5 \mathrm{~mL})$ and the combined organic phases were washed sequentially with $\mathrm{NaHCO}_{3}$ (sat. aq., $15 \mathrm{~mL}$ ) and brine (sat., $15 \mathrm{~mL}$ ). It was dried over $\mathrm{MgSO}_{4}$, and the solvent was removed under reduced pressure. Column chromatography (silica gel, nhexane/EtOAc 1:1) gave dankasterone B (2) (13.9 mg, $32.6 \mu \mathrm{mol}, 93 \%$ ) as colorless needles.

M.p.: $\quad 182-189^{\circ} \mathrm{C}\left(\mathrm{CHCl}_{3}\right)$.

TLC: $\quad R_{\mathrm{f}}=0.33($ nhexane/EtOAc $1: 1)$.

1H-NMR: $\quad\left(700 \mathrm{MHz}, \mathrm{CDCl}_{3}\right) ; \delta[\mathrm{ppm}]=5.28-5.19(\mathrm{~m}, 2 \mathrm{H}), 3.05(\mathrm{t}, J=9.9 \mathrm{~Hz}, 1 \mathrm{H}), 2.95(\mathrm{dd}, J=$ 13.2, $1.8 \mathrm{~Hz}, 1 \mathrm{H}), 2.90-2.87(\mathrm{~m}, 1 \mathrm{H}), 2.84(\mathrm{dt}, J=16.5,2.0 \mathrm{~Hz}, 1 \mathrm{H}), 2.79$ (ddd, $J=14.3$, 12.8, $5.9 \mathrm{~Hz}, 1 \mathrm{H}), 2.42(\mathrm{p}, J=6.9 \mathrm{~Hz}, 1 \mathrm{H}), 2.36$ (ddd, $J=12.7,4.2,2.5 \mathrm{~Hz}, 1 \mathrm{H}), 2.33-$ $2.26(\mathrm{~m}, 2 \mathrm{H}), 2.22(\mathrm{dt}, J=5.7,2.1 \mathrm{~Hz}, 1 \mathrm{H}), 2.20(\mathrm{~d}, J=6.1 \mathrm{~Hz}, 1 \mathrm{H}), 2.18-2.14(\mathrm{~m}, 1 \mathrm{H})$, $2.12-2.07(\mathrm{~m}, 1 \mathrm{H}), 2.02-1.93(\mathrm{~m}, 3 \mathrm{H}), 1.88-1.81(\mathrm{~m}, 1 \mathrm{H}), 1.69-1.61(\mathrm{~m}, 2 \mathrm{H}), 1.54$ (td, $J=12.9,5.7 \mathrm{~Hz}, 1 \mathrm{H}$ ), $1.48-1.42(\mathrm{~m}, 1 \mathrm{H}), 1.31$ (ddt, $J=13.3,6.8,2.4 \mathrm{~Hz}, 1 \mathrm{H}$ ), 1.27 (s, 3H), 1.15 (d, $J=7.1 \mathrm{~Hz}, 3 \mathrm{H}), 0.88$ (d, $J=6.8 \mathrm{~Hz}, 3 \mathrm{H}), 0.81$ (d, $J=6.7 \mathrm{~Hz}, 3 \mathrm{H}), 0.79$ (d, $J=6.8 \mathrm{~Hz}, 3 \mathrm{H}), 0.75(\mathrm{~s}, 3 \mathrm{H})$.

13C-NMR: $\quad\left(176 \mathrm{MHz}, \mathrm{CDCl}_{3}\right) ; \delta[\mathrm{ppm}]=214.9,208.6,207.7,135.4,132.2,65.8,60.2,53.4,50.2$, $45.6,43.4,40.7,40.1,38.7,37.0,36.9,35.9,34.2$, 33.2, 32.8, 27.4, 25.7, 24.3, 23.5, 20.2, 19.8, 17.7, 15.3.

IR: $\quad\left(\right.$ neat); $\tilde{v}\left[\mathrm{~cm}^{-1}\right]=2953(\mathrm{~m}), 2922(\mathrm{~s}), 2852(\mathrm{~m}), 1740(\mathrm{w}), 1459(\mathrm{~m}), 1377(\mathrm{w}), 1167(\mathrm{w})$, $722(\mathrm{w})$.

HRMS: (ESI-TOF); $\mathrm{m} / z$ calcd. for $\mathrm{C}_{28} \mathrm{H}_{42} \mathrm{O}_{3} \mathrm{Na}^{+}[\mathrm{M}+\mathrm{Na}]^{+}:$449.3026, found: 449.3030 .

Opt. act. $[\mathrm{a}]_{\mathrm{D}}^{24}=+28.2\left(c=1.00, \mathrm{CHCl}_{3}\right)$. 


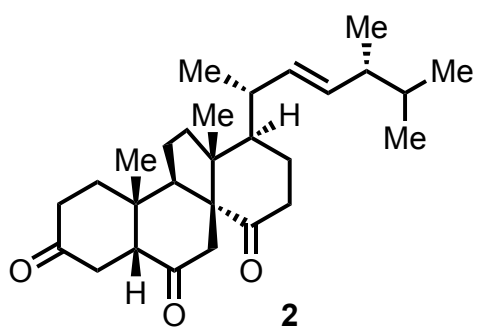

i) $\mathrm{KHMDS}, \mathrm{Et}_{3} \mathrm{~N}, \mathrm{TMSCl}$

$\mathrm{THF},-78^{\circ} \mathrm{C}, 30 \mathrm{~min}$

ii) $\mathrm{Pd}(\mathrm{OAc})_{2}$

$\stackrel{\mathrm{CH}_{3} \mathrm{CN}, 25^{\circ} \mathrm{C}, 16 \mathrm{~h}}{\longrightarrow}$

iii) $\mathrm{HCl}$

THF, $25^{\circ} \mathrm{C}, 24 \mathrm{~h}$

$36 \%, 90 \%$ brsm

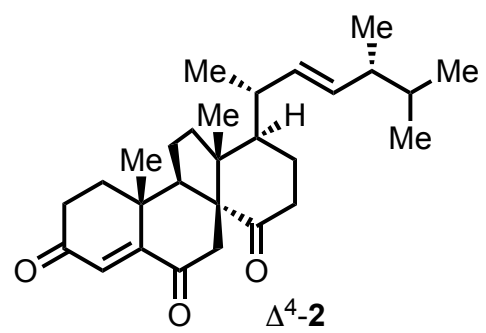

To a solution of dankasterone B (2) $\left(10 \mathrm{mg}, 23 \mu \mathrm{mol}, 1.0\right.$ eq.) in THF $(0.6 \mathrm{~mL})$ at $-78{ }^{\circ} \mathrm{C}$ were added $\mathrm{Et}_{3} \mathrm{~N}$ (64 $\mu \mathrm{L}, 0.46 \mathrm{mmol}, 20$ eq.), TMSCl (58 $\mu \mathrm{L}, 0.46 \mathrm{mmol}, 20$ eq.) and KHMDS (0.5 M in toluene, $140 \mu \mathrm{L}$, $70 \mu \mathrm{mol}, 3.0$ eq.) and the reaction mixture was stirred at this temperature for $30 \mathrm{~min}$. It was diluted with EtOAc ( $5 \mathrm{~mL}$ ) and phosphate buffer (aq., pH 7.4, $5 \mathrm{~mL}$ ) was added. The aqueous phase was extracted with EtOAc $(3 \times 10 \mathrm{~mL})$, the combined organic phases were washed with brine (sat., $20 \mathrm{~mL}$ ), dried over $\mathrm{Na}_{2} \mathrm{SO}_{4}$, and the solvent was removed under reduced pressure.

The residue was taken up in $\mathrm{CH}_{3} \mathrm{CN}(0.5 \mathrm{~mL}), \mathrm{Pd}(\mathrm{OAc})_{2}(26.0 \mathrm{mg}, 115 \mu \mathrm{mol}, 5.0$ eq.) was added and the reaction mixture was stirred at $25^{\circ} \mathrm{C}$ for $16 \mathrm{~h}$. It was filtered through Celite ${ }^{\circledR}$, rinsed with $\mathrm{CH}_{2} \mathrm{Cl}_{2}(15 \mathrm{~mL})$, and the solvent was removed under reduced pressure. The residue was taken up in THF $(1 \mathrm{~mL})$ and $\mathrm{HCl}$ ( $1 \mathrm{M}$ in $\mathrm{H}_{2} \mathrm{O}, 1 \mathrm{~mL}$ ) and stirred at $25^{\circ} \mathrm{C}$ for $24 \mathrm{~h}$. It was diluted with EtOAc $(5 \mathrm{~mL})$ and $\mathrm{H}_{2} \mathrm{O}(5 \mathrm{~mL})$ and the aqueous phase was extracted with EtOAc $(3 \times 10 \mathrm{~mL})$. The combined organic phases were washed with $\mathrm{NaHCO}_{3}$ (sat. aq., $20 \mathrm{~mL}$ ) and brine (sat., $20 \mathrm{~mL}$ ), dried over $\mathrm{MgSO}_{4}$ and the solvent was removed under reduced pressure. Column chromatography (silica gel, nhexane/EtOAc 3:1 $\rightarrow$ 1:1) gave dankasterone $A$ $\left(\Delta^{4}-2\right)(3.5 \mathrm{mg}, 8.2 \mu \mathrm{mol}, 36 \%)$ as a colorless solid and re-isolated dankasterone B (2) $(6.1 \mathrm{mg}, 14 \mu \mathrm{mol}$, $62 \%)$.

M.p.: $\quad 128-129^{\circ} \mathrm{C}\left(\mathrm{CH}_{3} \mathrm{OH}\right)$.

TLC: $\quad R_{\mathrm{f}}=0.27$ (nhexane/EtOAc 3:1).

1H-NMR: $\quad\left(700 \mathrm{MHz}, \mathrm{CDCl}_{3}\right) ; \delta[\mathrm{ppm}]=6.36(\mathrm{~d}, J=0.8 \mathrm{~Hz}, 1 \mathrm{H}), 5.31-5.22(\mathrm{~m}, 2 \mathrm{H}), 2.84-2.79(\mathrm{~m}$, 1H), 2.65 (dd, $J=16.8,1.6 \mathrm{~Hz}, 1 \mathrm{H}), 2.56-2.43(\mathrm{~m}, 5 \mathrm{H}), 2.41$ (td, $J=7.4,2.6 \mathrm{~Hz}, 1 \mathrm{H})$, $2.10-1.98(\mathrm{~m}, 3 \mathrm{H}), 1.92-1.82(\mathrm{~m}, 3 \mathrm{H}), 1.77(\mathrm{dt}, J=13.0,7.4 \mathrm{~Hz}, 1 \mathrm{H}), 1.74-1.66(\mathrm{~m}$, 2H), $1.50-1.46(\mathrm{~m}, 2 \mathrm{H}), 1.26(\mathrm{~s}, 3 \mathrm{H}), 1.09$ (d, $J=7.0 \mathrm{~Hz}, 3 \mathrm{H}), 0.98(\mathrm{~s}, 3 \mathrm{H}), 0.91$ (d, $J=$ $6.9 \mathrm{~Hz}, 3 \mathrm{H}), 0.83(\mathrm{~d}, J=6.8 \mathrm{~Hz}, 3 \mathrm{H}), 0.81(\mathrm{~d}, J=6.8 \mathrm{~Hz}, 3 \mathrm{H})$.

13C-NMR: $\quad\left(176 \mathrm{MHz}, \mathrm{CDCl}_{3}\right) ; \delta[\mathrm{ppm}]=214.9,200.1,199.2,156.2,135.3,132.5,126.7,62.3,54.1$, $49.5,49.5,43.4,41.0,39.1,38.5,38.1,37.4,36.2,34.5,33.2,25.3,24.2,23.8,23.3,20.2$, 19.8, 17.8, 17.2.

IR: $\quad$ (neat); $\tilde{v}\left[\mathrm{~cm}^{-1}\right]=2954(\mathrm{~m}), 2923(\mathrm{~s}), 2853(\mathrm{~m}), 1739(\mathrm{w}), 1698(\mathrm{w}), 1461(\mathrm{w}), 1377(\mathrm{w})$.

HRMS: (ESI-TOF); $m / z$ calcd. for $\mathrm{C}_{28} \mathrm{H}_{40} \mathrm{O}_{3} \mathrm{Na}^{+}[\mathrm{M}+\mathrm{Na}]^{+}: 447.2870$, found: 4472892. 
Opt. act. $\quad[a]_{D}^{21}=+43.3\left(c=0.7, \mathrm{CHCl}_{3}\right)$.

Periconiastone A (3)
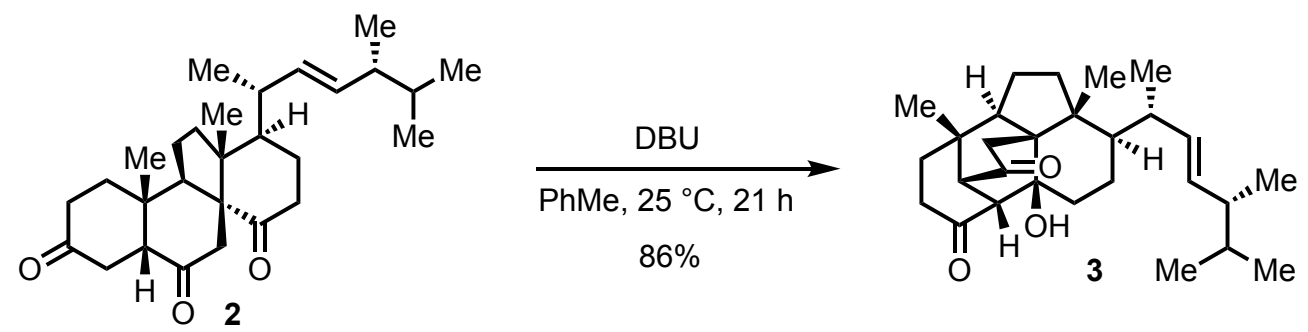

To a solution of dankasterone B (2) $(8.1 \mathrm{mg}, 19 \mu \mathrm{mol}, 1.0$ eq.) in toluene (1 mL) was added 1,8diazabicyclo[5.4.0]undec-7-ene ( $28 \mu \mathrm{L}, 190 \mu \mathrm{mol}, 10$ eq.) over $5 \mathrm{~h}$ at $25^{\circ} \mathrm{C}$ and the reaction mixture was stirred for $16 \mathrm{~h}$ at this temperature before $\mathrm{NH}_{4} \mathrm{Cl}$ (sat. aq., $10 \mathrm{~mL}$ ) was added. The aqueous phase was extracted with EtOAc $(3 \times 10 \mathrm{~mL})$ and the combined organic phases were washed sequentially with $\mathrm{HCl}$ ( $1 \mathrm{M}$ in $\mathrm{H}_{2} \mathrm{O}, 20 \mathrm{~mL}$ ), $\mathrm{NaHCO}_{3}$ (sat. aq., $20 \mathrm{~mL}$ ), and brine (sat., $20 \mathrm{~mL}$ ). The organic phase was dried over $\mathrm{MgSO}_{4}$ and the solvent was removed under reduced pressure. Column chromatography (silica gel, nhexane/EtOAc 1:1) gave periconiastone A (3) $(7.0 \mathrm{mg}, 16 \mu \mathrm{mol}, 86 \%)$ as colorless needles.

M.p. $\quad 175-176{ }^{\circ} \mathrm{C}($ EtOAc $)$

TLC: $\quad R_{\mathrm{f}}=0.16($ nhexane/EtOAc 3:1).

1H-NMR: $\quad\left(600 \mathrm{MHz}\right.$, pyridine- $\left.\mathrm{d}_{5}\right) ; \delta[\mathrm{ppm}]=6.64(\mathrm{~s}, 1 \mathrm{H}), 5.52(\mathrm{dd}, J=15.4,8.1 \mathrm{~Hz}, 1 \mathrm{H}), 5.30(\mathrm{dd}, J$ $=15.4,8.2 \mathrm{~Hz}, 1 \mathrm{H}), 3.21(\mathrm{~d}, J=18.5 \mathrm{~Hz}, 1 \mathrm{H}), 3.12(\mathrm{~d}, J=3.9 \mathrm{~Hz}, 1 \mathrm{H}), 2.87$ (ddd, $J=19.9$, $11.8,9.0 \mathrm{~Hz}, 1 \mathrm{H}), 2.80(\mathrm{t}, J=10.0 \mathrm{~Hz}, 1 \mathrm{H}), 2.52-2.42(\mathrm{~m}, 3 \mathrm{H}), 2.16-2.10(\mathrm{~m}, 2 \mathrm{H}), 2.03$ (ddd, $J=14.2,9.7,5.3 \mathrm{~Hz}, 1 \mathrm{H}), 1.98-1.91(\mathrm{~m}, 1 \mathrm{H}), 1.90-1.77(\mathrm{~m}, 5 \mathrm{H}), 1.61-1.41(\mathrm{~m}$, $5 \mathrm{H}), 1.22(\mathrm{~s}, 3 \mathrm{H}), 1.13(\mathrm{~d}, J=6.9 \mathrm{~Hz}, 3 \mathrm{H}), 0.99(\mathrm{~s}, 3 \mathrm{H}), 0.92(\mathrm{~d}, J=6.8 \mathrm{~Hz}, 3 \mathrm{H}), 0.87-$ $0.83(\mathrm{~m}, 6 \mathrm{H})$.

13C-NMR: $\quad\left(151 \mathrm{MHz}\right.$, pyridine- $\left.\mathrm{d}_{5}\right) ; \delta[\mathrm{ppm}]=213.1,209.2,134.6,134.5,73.1,65.3,58.8,56.0,48.2$, $47.8,44.0,42.3,38.5,37.6,37.4,37.4,36.8,34.3,33.9,33.6,26.5,24.4,22.7,20.7,20.6$, 20.3, 19.3, 18.3 .

IR: $\quad$ (neat); $\tilde{v}\left[\mathrm{~cm}^{-1}\right]=3456(\mathrm{br} \mathrm{w}), 2956(\mathrm{~s}), 2925(\mathrm{~s}), 2871(\mathrm{~m}), 1718(\mathrm{~s}), 1685(\mathrm{~s}), 1455(\mathrm{w})$, $1384(w), 1206(w), 956(w), 761(w)$.

HRMS: (ESI-TOF); $\mathrm{m} / \mathrm{z}$ calcd. for $\mathrm{C}_{28} \mathrm{H}_{42} \mathrm{O}_{3} \mathrm{Na}^{+}[\mathrm{M}+\mathrm{Na}]^{+}:$449.3026, found: 449.3034 .

Opt. act. $[a]_{D}^{21}=-8.8\left(c=0.1, \mathrm{CH}_{3} \mathrm{OH}\right)$. 


\subsection{Synthesis of Sulfone 22}

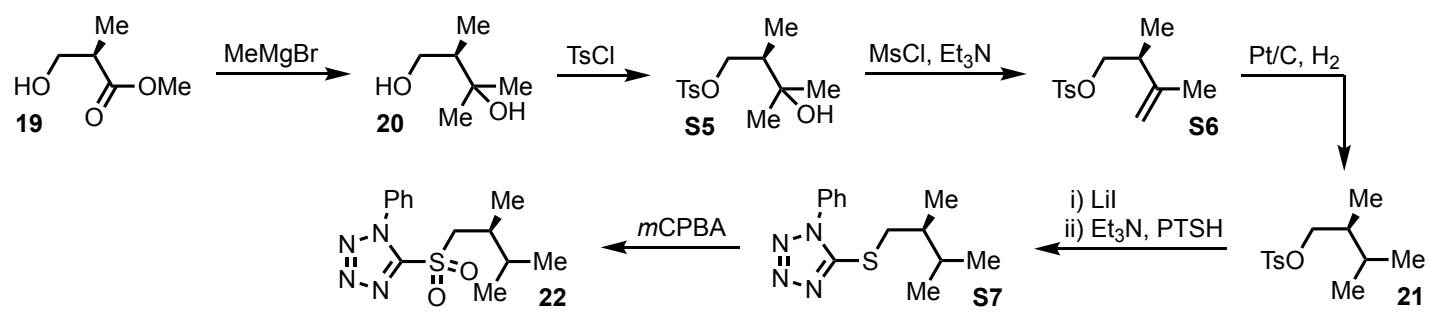

(R)-2,3-Dimethylbutane-1,3-diol (20)
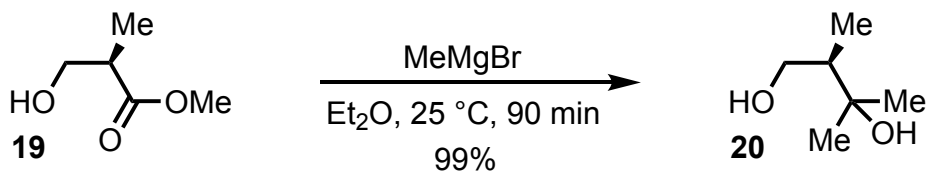

To a solution of $(R)$-Roche ester (19) (180 mg, $1.52 \mathrm{mmol}, 1.0$ eq.) in $\mathrm{Et}_{2} \mathrm{O}$ (5 mL) was added methylmagnesium bromide ( $3 \mathrm{M}$ in $\mathrm{Et}_{2} \mathrm{O}, 9.3 \mathrm{~mL}, 28 \mathrm{mmol}, 3.3$ eq.) at $0{ }^{\circ} \mathrm{C}$ and the reaction mixture was stirred at $25^{\circ} \mathrm{C}$ for $1.5 \mathrm{~h}$. It was cooled to $0{ }^{\circ} \mathrm{C}$ and $\mathrm{HCl}\left(1 \mathrm{M}\right.$ in $\left.\mathrm{H}_{2} \mathrm{O}, 10 \mathrm{~mL}\right)$ and $\mathrm{Et}_{2} \mathrm{O}(10 \mathrm{~mL})$ were added dropwise. The aqueous phase was neutralized and a continuous extraction with $\mathrm{Et}_{2} \mathrm{O}(250 \mathrm{~mL})$ was performed for $48 \mathrm{~h}$. The solvent was removed under reduced pressure giving diol 20 (178 mg, $1.51 \mathrm{mmol}$, $99 \%$ ) as a colorless oil, which was used in the next step without further purification.

TLC: $\quad R_{\mathrm{f}}=0.28\left(\right.$ npentane/Et $\left.\mathrm{E}_{2} \mathrm{O} 1: 2\right)$.

1H-NMR: $\quad\left(500 \mathrm{MHz}, \mathrm{CDCl}_{3}\right) ; \delta[\mathrm{ppm}]=3.89(\mathrm{br} \mathrm{s}, 1 \mathrm{H}), 3.73-3.56(\mathrm{~m}, 3 \mathrm{H}), 1.84-1.71(\mathrm{~m}, 1 \mathrm{H}), 1.22$ (s, 3H), $1.15(\mathrm{~s}, 3 \mathrm{H}), 0.82(\mathrm{~d}, J=7.1 \mathrm{~Hz}, 3 \mathrm{H})$.

13C-NMR: $\quad\left(126 \mathrm{MHz}, \mathrm{CDCl}_{3}\right) ; \delta[\mathrm{ppm}]=74.7,66.2,44.0,29.8,24.1,13.1$.

IR: $\quad$ (neat); $\tilde{v}\left[\mathrm{~cm}^{-1}\right]=3335(\mathrm{br} \mathrm{m}), 2973(\mathrm{~m}), 2934(\mathrm{w}), 2885(\mathrm{w}), 1717(\mathrm{w}), 1458(\mathrm{~m}), 1366(\mathrm{~m})$, $1174(\mathrm{~m}), 1024(\mathrm{~s}), 946(\mathrm{~m}), 904(\mathrm{~m}), 857(\mathrm{w})$.

HRMS: $\quad$ (ESI-TOF); $\mathrm{m} / z$ calcd. for $\mathrm{C}_{6} \mathrm{H}_{14} \mathrm{O}_{2} \mathrm{Na}^{+}[\mathrm{M}+\mathrm{Na}]+:$ 141.0886, found: 141.0898 .

Opt. act. $\quad[\mathrm{a}]_{\mathrm{D}}^{26}=-4.0\left(c=1.00, \mathrm{CHCl}_{3}\right)$.

(R)-3-Hydroxy-2,3-dimethylbutyl 4-methylbenzenesulfonate (S5)
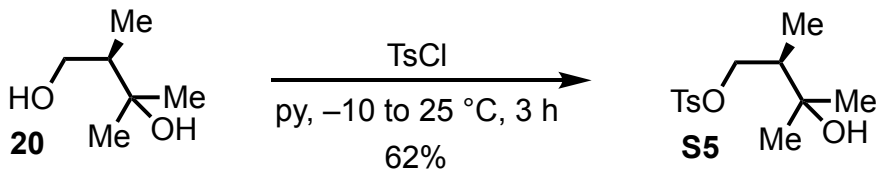
To a solution of diol 20 (200 mg, $1.69 \mathrm{mmol}, 1.0$ eq.) in pyridine $(1.5 \mathrm{~mL})$ at $-10^{\circ} \mathrm{C}$ was added tosyl chloride ( $806 \mathrm{mg}, 4.23 \mathrm{mmol}, 2.5$ eq.) and the reaction mixture was allowed to warm to $25^{\circ} \mathrm{C}$ over $4 \mathrm{~h}$. A mixture of ice and $\mathrm{H}_{2} \mathrm{O}(5 \mathrm{~mL})$ was added and the aqueous phase was extracted with $\mathrm{Et}_{2} \mathrm{O}(3 \times 15 \mathrm{~mL})$. The combined organic phases were washed sequentially with $\mathrm{H}_{2} \mathrm{O}(25 \mathrm{~mL}), \mathrm{HCl}\left(1 \mathrm{M} \mathrm{in} \mathrm{H}_{2} \mathrm{O}, 25 \mathrm{~mL}\right.$ ), and brine (sat., $25 \mathrm{~mL}$ ), dried over $\mathrm{MgSO}_{4}$, and the solvent was removed under reduced pressure. Column chromatography (silica gel, nhexane/EtOAc 3:1) gave toslyate S5 (287 mg, $1.06 \mathrm{mmol}, 62 \%$ ) as a colorless oil.

TLC: $\quad R_{\mathrm{f}}=0.29($ nhexane/EtOAc $3: 1)$.

1H-NMR: $\quad\left(500 \mathrm{MHz}, \mathrm{CDCl}_{3}\right) ; \delta[p p m]=7.78(\mathrm{~d}, J=8.1 \mathrm{~Hz}, 2 \mathrm{H}), 7.34(\mathrm{~d}, J=8.1 \mathrm{~Hz}, 2 \mathrm{H}), 4.25-4.21$ (m, 1H), $3.93-3.89(\mathrm{~m}, 1 \mathrm{H}), 2.44(\mathrm{~s}, 3 \mathrm{H}), 1.90-1.77(\mathrm{~m}, 1 \mathrm{H}), 1.54-1.42(\mathrm{~m}, 1 \mathrm{H}), 1.18$ (s, 3H), $1.11(\mathrm{~s}, 3 \mathrm{H}), 0.95(\mathrm{~d}, J=7.0 \mathrm{~Hz}, 3 \mathrm{H})$.

${ }^{13} \mathrm{C}$-NMR: $\quad\left(126 \mathrm{MHz}, \mathrm{CDCl}_{3}\right) ; \delta[\mathrm{ppm}]=144.9,133.1,130.0,128.0,72.7,72.1,43.5,28.7,26.2,21.8$, 12.8.

IR: $\quad$ (neat); $\tilde{v}\left[\mathrm{~cm}^{-1}\right]=3541(\mathrm{br} \mathrm{w}), 2974(\mathrm{w}), 2925(\mathrm{w}), 2857(\mathrm{w}), 1598(\mathrm{w}), 1463(\mathrm{w}), 1353(\mathrm{~m})$, $1173(\mathrm{~s}), 1097(\mathrm{~m}), 950(\mathrm{~s}), 837(\mathrm{~m}), 813(\mathrm{~m}), 735(\mathrm{~m})$.

HRMS: $\quad$ (ESI-TOF); $\mathrm{m} / \mathrm{z}$ calcd. for $\mathrm{C}_{13} \mathrm{H}_{20} \mathrm{O}_{4} \mathrm{SNa}^{+}[\mathrm{M}+\mathrm{Na}]^{+}:$295.0975, found: 295.0987.

Opt. act. $[\mathrm{a}]_{\mathrm{D}}^{26}=+17.4\left(c=1.00, \mathrm{CHCl}_{3}\right)$.

(S)-2,3-Dimethylbut-3-en-1-yl 4-methylbenzenesulfonate (S6)
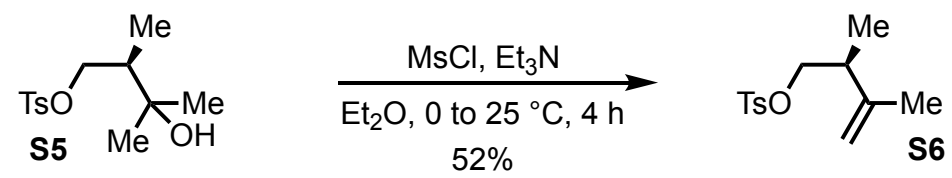

To a solution of tosylate $\mathrm{S} 5\left(4.21 \mathrm{~g}, 15.5 \mathrm{mmol}, 1.0\right.$ eq.) in $\mathrm{Et}_{2} \mathrm{O}(300 \mathrm{~mL})$ at $25^{\circ} \mathrm{C}$ was added methanesulfonyl chloride $\left(6.0 \mathrm{~mL}, 77 \mathrm{mmol}, 5.0\right.$ eq.). It was cooled to $0{ }^{\circ} \mathrm{C}$, triethylamine $(43 \mathrm{~mL}, 0.31 \mathrm{~mol}$, 20 eq.) was added dropwise and the reaction mixture was stirred at $25^{\circ} \mathrm{C}$ for $4 \mathrm{~h}$. $\mathrm{HCl}\left(20 \%\right.$ in $\mathrm{H}_{2} \mathrm{O} w / w$, $6 \mathrm{~mL}$ ) was added at $0{ }^{\circ} \mathrm{C}$ and the organic phase was separated. The aqueous phase was extracted with $\mathrm{Et}_{2} \mathrm{O}(3 \times 15 \mathrm{~mL})$ and the combined organic phases were washed sequentially with $\mathrm{HCl}\left(1 \mathrm{M} \mathrm{in} \mathrm{H}_{2} \mathrm{O}, 25 \mathrm{~mL}\right)$, $\mathrm{NaHCO}_{3}$ (sat. aq., $25 \mathrm{~mL}$ ), and brine (sat., $25 \mathrm{~mL}$ ). The organic phase was dried over $\mathrm{MgSO}_{4}$ and the solvent was removed under reduced pressure. Column chromatography (silica gel, nhexane/EtOAc 12:1 $\rightarrow$ 9:1) gave unsaturated tosylate $\mathbf{S 6}(2.03 \mathrm{~g}, 8.01 \mathrm{mmol}, 52 \%)$ as a colorless oil.

TLC: $\quad R_{\mathrm{f}}=0.28($ nhexane/EtOAc 50:1). 
1H-NMR: $\quad\left(500 \mathrm{MHz}, \mathrm{CDCl}_{3}\right) ; \delta[\mathrm{ppm}]=7.78(\mathrm{~d}, J=8.0 \mathrm{~Hz}, 2 \mathrm{H}), 7.34(\mathrm{~d}, J=8.0 \mathrm{~Hz}, 2 \mathrm{H}), 4.79-4.76$ $(\mathrm{m}, 1 \mathrm{H}), 4.69-4.68(\mathrm{~m}, 1 \mathrm{H}), 3.98(\mathrm{dd}, J=9.5,6.6 \mathrm{~Hz}, 1 \mathrm{H}), 3.86(\mathrm{dd}, J=9.5,7.2 \mathrm{~Hz}, 1 \mathrm{H})$, $2.50-2.45(\mathrm{~m}, 1 \mathrm{H}), 2.44(\mathrm{~s}, 3 \mathrm{H}), 1.61(\mathrm{~s}, 3 \mathrm{H}), 1.01(\mathrm{~d}, J=7.0 \mathrm{~Hz}, 3 \mathrm{H})$.

13C-NMR: $\quad\left(126 \mathrm{MHz}, \mathrm{CDCl}_{3}\right) ; \delta[\mathrm{ppm}]=145.0,144.8,133.3,129.9,128.0,112.1,73.2,40.2,21.7$, 20.2, 16.0 .

IR: $\quad\left(\right.$ neat); $\tilde{v}\left[\mathrm{~cm}^{-1}\right]=2972(\mathrm{w}), 1650(\mathrm{w}), 1598(\mathrm{w}), 1454(\mathrm{w}), 1357(\mathrm{~m}), 1174(\mathrm{~s}), 1096(\mathrm{~m})$, $966(\mathrm{~s}), 895(\mathrm{~m}), 834(\mathrm{~m}), 813(\mathrm{~s}), 767(\mathrm{~m})$.

HRMS: (ESI-TOF); $\mathrm{m} / \mathrm{z}$ calcd. for $\mathrm{C}_{13} \mathrm{H}_{18} \mathrm{O}_{3} \mathrm{SNa}^{+}[\mathrm{M}+\mathrm{Na}]^{+}: 277.0869$, found: 277.0885 .

Opt. act. $[a]_{D}^{26}=+6.9\left(c=1.00, \mathrm{CHCl}_{3}\right)$.

(S)-2,3-Dimethylbutyl 4-methylbenzenesulfonate (21)
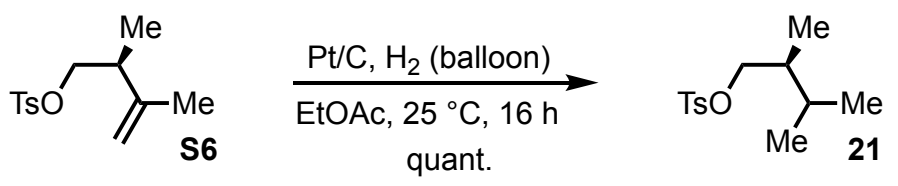

To a solution of olefin $\mathbf{S} 6$ (0.99 g, $3.9 \mathrm{mmol}, 1.0$ eq.) in EtOAc $(40 \mathrm{~mL})$ was added Pt/C (10\% w/w, $767 \mathrm{mg}$, $390 \mu \mathrm{mol}, 0.1$ eq.), the atmosphere was exchanged to hydrogen, and the reaction mixture was stirred at 25 ${ }^{\circ} \mathrm{C}$ for $18 \mathrm{~h}$. It was filtered through $\mathrm{Celite}^{\circledR}$ and the solvent was removed under reduced pressure. Tosylate 21 (925 mg, $3.90 \mathrm{mmol}$, quant.) was obtained as a colorless oil and used in the next step without further purification.

The enantiomeric excess was determined by HPLC using a Chiralpak ${ }^{\circledR}$ IC column, $\lambda=210.4 \mathrm{~nm}$, nhexane/PrOH 199:1, flow rate $=1 \mathrm{~mL} / \mathrm{min} ; \mathrm{t}_{\mathrm{R}}=14.47 \mathrm{~min}$ (minor, epi-21, not shown), 15.27 min (major, 21) $(e e>99 \%)$.

HPLC traces of racemic tosylate $\mathbf{2 1}_{\text {(rac) }}$ (top) and enantiopure tosylate $\mathbf{2 1}$ (bottom)

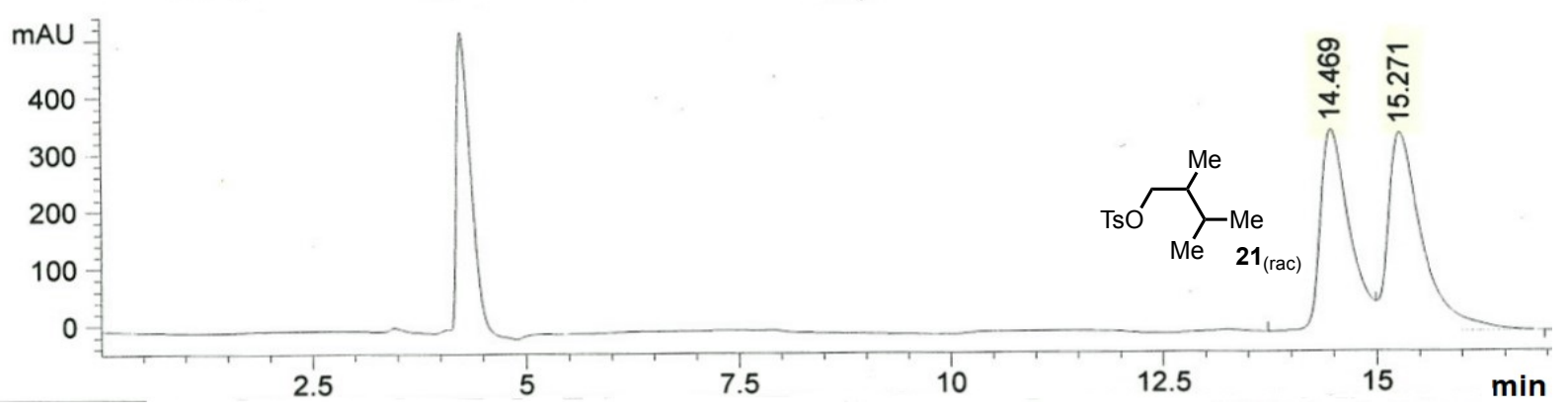




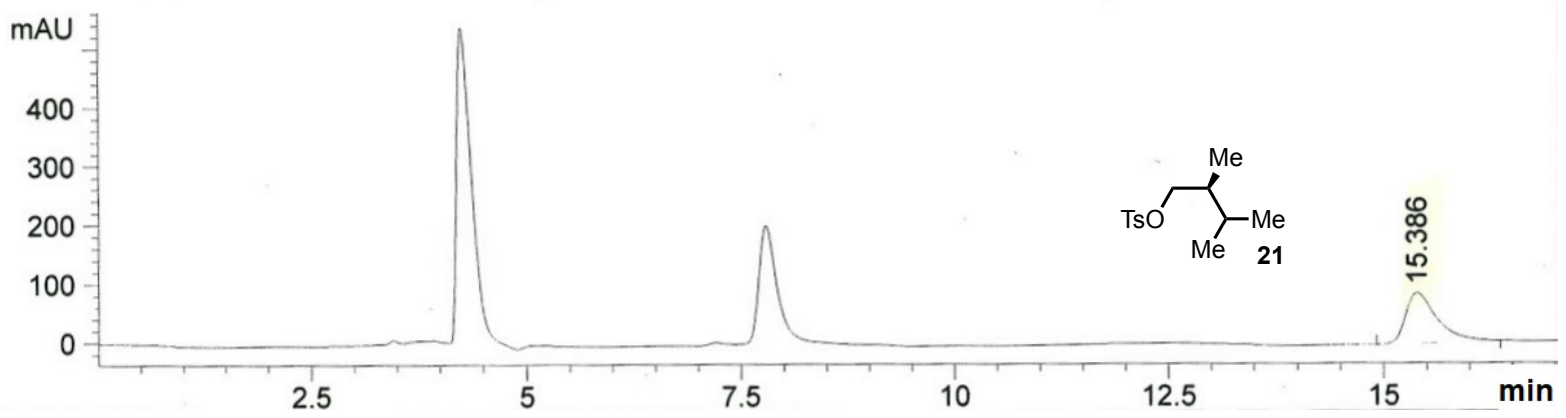

TLC: $\quad R_{\mathrm{f}}=0.30($ nhexane/EtOAc 50:1).

1H-NMR: $\quad\left(400 \mathrm{MHz}, \mathrm{CDCl}_{3}\right) ; \delta[\mathrm{ppm}]=7.79(\mathrm{~d}, J=8.1 \mathrm{~Hz}, 2 \mathrm{H}), 7.34(\mathrm{~d}, J=8.1 \mathrm{~Hz}, 2 \mathrm{H}), 3.94(\mathrm{dd}, J$ = 9.4, $5.7 \mathrm{~Hz}, 1 \mathrm{H}), 3.84(\mathrm{dd}, J=9.4,6.5 \mathrm{~Hz}, 1 \mathrm{H}), 2.45(\mathrm{~s}, 3 \mathrm{H}), 1.73-1.59(\mathrm{~m}, 2 \mathrm{H}), 0.87-$ $0.79(\mathrm{~m}, 6 \mathrm{H}), 0.76(\mathrm{~d}, J=6.6 \mathrm{~Hz}, 3 \mathrm{H})$.

13C-NMR: $\quad\left(101 \mathrm{MHz}, \mathrm{CDCl}_{3}\right) ; \delta[\mathrm{ppm}]=144.8,133.3,129.9,128.0,73.9,38.5,28.9,21.8,20.3,18.0$, 12.7.

IR: $\quad\left(\right.$ neat); $\tilde{v}\left[\mathrm{~cm}^{-1}\right]=2961(\mathrm{w}), 1598(\mathrm{w}), 1466(\mathrm{w}), 1358(\mathrm{~m}), 1174(\mathrm{~s}), 1097(\mathrm{~m}), 960(\mathrm{~s}), 929$ $(\mathrm{m}), 837(\mathrm{~m}), 813(\mathrm{~m}), 759(\mathrm{~m})$.

HRMS: $\quad$ (ESI-TOF); $\mathrm{m} / z$ calcd. for $\mathrm{C}_{13} \mathrm{H}_{20} \mathrm{O}_{3} \mathrm{SNa}^{+}[\mathrm{M}+\mathrm{Na}]^{+}:$279.1025, found: 279.1026.

Opt. act. $[\mathrm{a}]_{\mathrm{D}}^{26}=+5.2\left(c=1.00, \mathrm{CHCl}_{3}\right)$.

(S)-5-((2,3-Dimethylbutyl)thio)-1-phenyl-1H-tetrazole (S7)
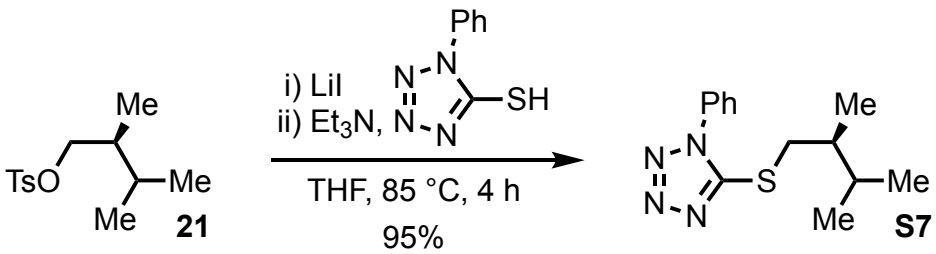

To a solution of tosylate 21 (462 mg, $1.80 \mathrm{mmol}, 1.0$ eq.) in THF (20 mL) was added lithium iodide (previously dried under reduced pressure at $120^{\circ} \mathrm{C}$ for $24 \mathrm{~h}, 522 \mathrm{mg}, 3.60 \mathrm{mmol}, 2.0$ eq.) and the reaction mixture was heated to reflux for $1 \mathrm{~h}$. It was cooled to $25^{\circ} \mathrm{C}$ and triethylamine $(810 \mu \mathrm{L}, 5.85 \mathrm{mmol}, 3.0 \mathrm{eq}$.) and 1-phenyl-1 $\mathrm{H}$-tetrazole-5-thiol $(729 \mathrm{mg}, 3.60 \mathrm{mmol}, 2.0 \mathrm{eq}$.) were added before the solution was again heated to reflux for $3 \mathrm{~h}$ and then stirred at $25^{\circ} \mathrm{C}$ for $16 \mathrm{~h}$. The precipitate was filtered off, the solvent was removed under reduced pressure, and the resulting residue was taken up in $\mathrm{Et}_{2} \mathrm{O}(20 \mathrm{~mL})$ and brine (sat., $20 \mathrm{~mL})$. The aqueous phase was extracted with $\mathrm{Et}_{2} \mathrm{O}(3 \times 15 \mathrm{~mL})$ and the combined organic phases were washed with brine (sat., $20 \mathrm{~mL}$ ), dried over $\mathrm{MgSO}_{4}$, and the solvent was removed under reduced pressure. Column chromatography (silica gel, nhexane/EtOAc 12:1) gave thioether $\mathbf{S 7}$ (448 mg, $1.71 \mathrm{mmol}, 95 \%$ ) as a colorless oil. 
TLC: $\quad R_{\mathrm{f}}=0.35$ (nhexane/EtOAc 9:1).

1H-NMR: $\quad\left(500 \mathrm{MHz}, \mathrm{CDCl}_{3}\right) ; \delta[\mathrm{ppm}]=7.61-7.50(\mathrm{~m}, 5 \mathrm{H}), 3.52(\mathrm{dd}, J=12.5,5.3 \mathrm{~Hz}, 1 \mathrm{H}), 3.23(\mathrm{dd}$, $J=12.6,8.3 \mathrm{~Hz}, 1 \mathrm{H}), 1.85-1.72(\mathrm{~m}, 2 \mathrm{H}), 0.98(\mathrm{~d}, J=6.8 \mathrm{~Hz}, 3 \mathrm{H}), 0.95(\mathrm{~d}, J=6.7 \mathrm{~Hz}$, $3 \mathrm{H}), 0.89(\mathrm{~d}, J=6.7 \mathrm{~Hz}, 3 \mathrm{H})$.

13C-NMR: $\quad\left(126 \mathrm{MHz}, \mathrm{CDCl}_{3}\right) ; \delta[\mathrm{ppm}]=154.9,134.0,130.2,129.9,124.0,38.8,38.6,31.6,20.4$, 17.8, 15.1.

IR: $\quad$ (neat); $\tilde{v}\left[\mathrm{~cm}^{-1}\right]=2958(\mathrm{~m}), 2924(\mathrm{~m}), 2872(\mathrm{w}), 2853(\mathrm{w}), 1735(\mathrm{w}), 1597(\mathrm{w}), 1499(\mathrm{~m})$, $1463(\mathrm{~m}), 1385(\mathrm{~m}), 1239(\mathrm{~m}), 1974(\mathrm{w}), 1014(\mathrm{~m}), 978(\mathrm{w}), 912(\mathrm{w}), 759(\mathrm{~s}), 733(\mathrm{~m})$.

HRMS: $\quad$ (ESI-TOF); $\mathrm{m} / \mathrm{z}$ calcd. for $\mathrm{C}_{13} \mathrm{H}_{18} \mathrm{~N}_{4} \mathrm{SNa}^{+}[\mathrm{M}+\mathrm{Na}]+:$ 285.1144, found: 285.1162 .

Opt. act. $[\mathrm{a}]_{\mathrm{D}}^{26}=+13.4\left(c=0.92, \mathrm{CHCl}_{3}\right)$.

(S)-5-((2,3-Dimethylbutyl)sulfonyl)-1-phenyl-1H-tetrazole (22)

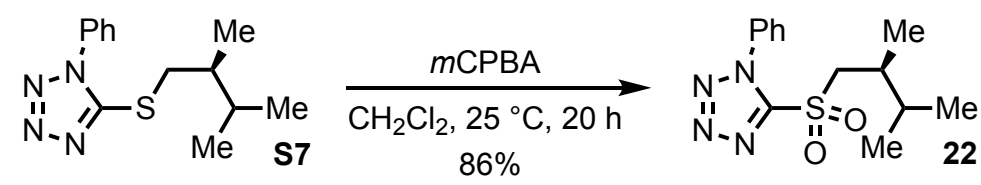

To a solution of thioether $\mathbf{S} 7$ (407 mg, $1.55 \mathrm{mmol}, 1.0$ eq.) in $\mathrm{CH}_{2} \mathrm{Cl}_{2}(16 \mathrm{~mL})$ was added $m \mathrm{CPBA}(70 \% w / w$, $804 \mathrm{mg}, 4.66 \mathrm{mmol}, 3.0 \mathrm{eq}$.) and the reaction was stirred at $25^{\circ} \mathrm{C}$ for $24 \mathrm{~h}$. The reaction mixture was filtered, the filtrate was cooled to $0^{\circ} \mathrm{C}$ and $\mathrm{H}_{2} \mathrm{O}(3 \mathrm{~mL}), \mathrm{NaHSO}_{3}\left(5 \%\right.$ aq., $15 \mathrm{~mL}$ ) and $\mathrm{NaHCO}_{3}$ (sat. aq., $15 \mathrm{~mL}$ ) were added. The aqueous phase was extracted with $\mathrm{CH}_{2} \mathrm{Cl}_{2}(3 \times 15 \mathrm{~mL})$ and the combined organic phases were washed sequentially with $\mathrm{NaHCO}_{3}$ (sat. aq., $15 \mathrm{~mL}$ ) and brine (sat., $15 \mathrm{~mL}$ ). The organic phase was dried over $\mathrm{MgSO}_{4}$ and the solvent was removed under reduced pressure. Column chromatography (silica gel, nhexane/EtOAc 9:1) gave sulfone 22 (393 mg, $1.34 \mathrm{mmol}, 86 \%$ ) as a colorless oil.

TLC: $\quad R_{\mathrm{f}}=0.32($ nhexane/EtOAc $12: 1)$.

1H-NMR: $\quad\left(500 \mathrm{MHz}, \mathrm{CDCl}_{3}\right) ; \delta[\mathrm{ppm}]=7.70-7.66(\mathrm{~m}, 2 \mathrm{H}), 7.65-7.57(\mathrm{~m}, 3 \mathrm{H}), 3.85(\mathrm{dd}, J=14.4$, $3.7 \mathrm{~Hz}, 1 \mathrm{H}), 3.52(\mathrm{dd}, J=14.4,9.0 \mathrm{~Hz}, 1 \mathrm{H}), 2.32-2.23(\mathrm{~m}, 1 \mathrm{H}), 1.89-1.80(\mathrm{~m}, 1 \mathrm{H}), 1.10$ $(\mathrm{d}, J=6.9 \mathrm{~Hz}, 3 \mathrm{H}), 0.93(\mathrm{~d}, J=6.8 \mathrm{~Hz}, 3 \mathrm{H}), 0.90(\mathrm{~d}, J=6.9 \mathrm{~Hz}, 3 \mathrm{H})$.

13C-NMR: $\quad\left(126 \mathrm{MHz}, \mathrm{CDCl}_{3}\right) ; \delta[\mathrm{ppm}]=154.2,133.2,131.6,129.8,125.3,60.2,33.4,32.5,19.5$, $17.8,15.8$.

IR: $\quad\left(\right.$ neat); $\tilde{v}\left[\mathrm{~cm}^{-1}\right]=2960(\mathrm{~m}), 2923(\mathrm{~m}), 2853(\mathrm{~m}), 1497(\mathrm{~m}), 1462(\mathrm{~m}), 1336(\mathrm{~s}), 1150(\mathrm{~s})$, 763 (s).

HRMS: $\quad$ (ESI-TOF); $\mathrm{m} / z$ calcd. for $\mathrm{C}_{13} \mathrm{H}_{18} \mathrm{~N}_{4} \mathrm{O}_{2} \mathrm{SNa}^{+}[\mathrm{M}+\mathrm{Na}]^{+}: 317.1043$, found: 317.1064 .

Opt. act. $[a]_{D}^{27}=+8.1\left(c=1.00, \mathrm{CHCl}_{3}\right)$. 


\subsection{Synthesis of Swinhoeisterol A (1)}

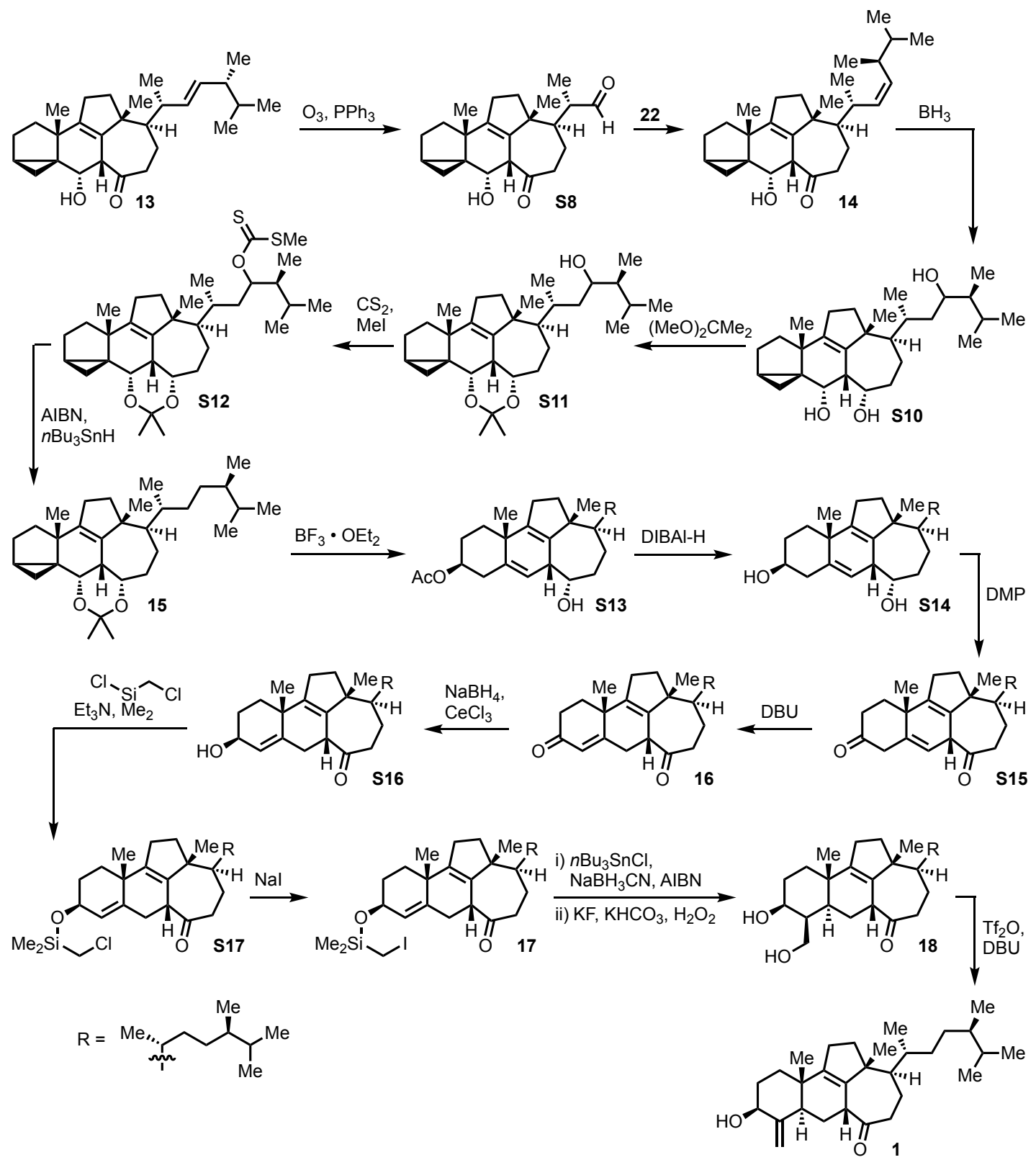

(22E)-6a-Hydroxy-13(14 $\rightarrow 8), 14(8 \rightarrow 7)$ diabeo-3a,5-cyclo-5a,7 $\beta(H)$-ergosta-8,22-dien-14-one (13)
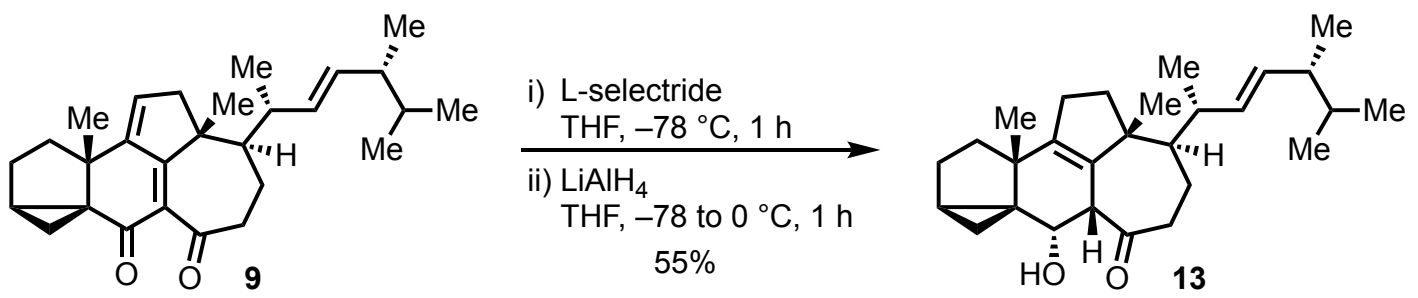
A solution of diene dione 9 (1,31 g, $3.23 \mathrm{mmol}, 1.0 \mathrm{eq}$.) in THF (32 mL) was degassed applying three freezepump-thaw cycles. After cooling to $-78^{\circ} \mathrm{C}$, L-selectride (1 $\mathrm{M}$ in THF, $6.5 \mathrm{~mL}, 6.5 \mathrm{mmol}$, 2.0 eq.) was added dropwise over $30 \mathrm{~min}$ and the resulting solution was stirred at this temperature for $30 \mathrm{~min}$. Lithium aluminum hydride (1 $\mathrm{M}$ in THF, $6.5 \mathrm{~mL}, 6.5 \mathrm{mmol}, 2.0$ eq.) was added dropwise over $30 \mathrm{~min}$, and the reaction mixture was warmed to $0{ }^{\circ} \mathrm{C}$. After stirring for $30 \mathrm{~min}$ at this temperature, EtOAc $(20 \mathrm{~mL})$ and Rochelle's salt $(1 / 2$ sat. aq., $30 \mathrm{~mL}$ ) were added carefully, and the mixture was vigorously stirred at $25^{\circ} \mathrm{C}$ for $16 \mathrm{~h}$. A solution of $\mathrm{NaBO}_{3} \cdot 4 \mathrm{H}_{2} \mathrm{O}\left(1.24 \mathrm{~g}, 8.08 \mathrm{mmol}, 2.5\right.$ eq.) in $\mathrm{H}_{2} \mathrm{O}(10 \mathrm{~mL})$ was added and stirring was continued for 30 min. The aqueous phase was extracted with EtOAc $(3 \times 25 \mathrm{~mL})$ and the combined organic phases were washed with brine (sat., $50 \mathrm{~mL}$ ), dried over $\mathrm{MgSO}_{4}$, and the solvent was removed under reduced pressure. Column chromatography (silica gel, nhexane/EtOAc 50:1 $\rightarrow$ 19:1) gave $\beta$-hydroxy ketone 13 (731 mg, 1.78 mmol, $55 \%)$ as a colorless oil.

TLC: $\quad R_{\mathrm{f}}=0.44($ nhexane/EtOAc 9:1).

1H-NMR: $\quad\left(500 \mathrm{MHz}, \mathrm{CDCl}_{3}\right) ; \delta[\mathrm{ppm}]=5.31-5.21(\mathrm{~m}, 2 \mathrm{H}), 4.44(\mathrm{~d}, J=8.5 \mathrm{~Hz}, 1 \mathrm{H}), 4.25-4.19(\mathrm{~m}$, $1 \mathrm{H}), 3.16(\mathrm{~d}, J=6.4 \mathrm{~Hz}, 1 \mathrm{H}), 2.60-2.52(\mathrm{~m}, 1 \mathrm{H}), 2.51-2.40(\mathrm{~m}, 2 \mathrm{H}), 2.35-2.26(\mathrm{~m}$, $1 \mathrm{H}), 2.21-2.13(\mathrm{~m}, 2 \mathrm{H}), 2.12-2.06(\mathrm{~m}, 1 \mathrm{H}), 1.87-1.82(\mathrm{~m}, 1 \mathrm{H}), 1.74-1.67(\mathrm{~m}, 1 \mathrm{H})$, $1.66-1.62(\mathrm{~m}, 1 \mathrm{H}), 1.62-1.58(\mathrm{~m}, 2 \mathrm{H}), 1.58-1.54(\mathrm{~m}, 1 \mathrm{H}), 1.53-1.47(\mathrm{~m}, 2 \mathrm{H}), 1.46-$ $1.39(\mathrm{~m}, 2 \mathrm{H}), 1.09(\mathrm{~s}, 3 \mathrm{H}), 1.05(\mathrm{~d}, J=7.1 \mathrm{~Hz}, 3 \mathrm{H}), 0.98(\mathrm{~s}, 3 \mathrm{H}), 0.90(\mathrm{~d}, J=6.8 \mathrm{~Hz}, 3 \mathrm{H})$, $0.82(\mathrm{~d}, J=6.7 \mathrm{~Hz}, 3 \mathrm{H}), 0.80(\mathrm{~d}, J=6.8 \mathrm{~Hz}, 3 \mathrm{H}), 0.77-0.73(\mathrm{~m}, 1 \mathrm{H}), 0.31(\mathrm{t}, J=4.2 \mathrm{~Hz}$, $1 \mathrm{H})$.

${ }^{13} \mathrm{C}-N M R: \quad\left(126 \mathrm{MHz}, \mathrm{CDCl}_{3}\right) ; \delta[\mathrm{ppm}]=219.7,144.8,137.6,134.9,132.3,68.2,53.6,53.5,48.3$, $46.1,45.3,43.5,38.5,37.9,37.8,33.2,33.1,28.1,26.6,22.9,22.6,21.5,20.9,20.2,19.9$, 19.9, 17.8, 7.7.

IR: $\quad$ (neat); $\tilde{v}\left[\mathrm{~cm}^{-1}\right]=3441(\mathrm{br} \mathrm{w}), 2955(\mathrm{~s}), 2924(\mathrm{~s}), 2867(\mathrm{~m}), 1738(\mathrm{w}), 1684(\mathrm{~m}), 1457(\mathrm{~m})$, $1375(w), 1070(w)$.

HRMS: (ESI-TOF); $\mathrm{m} / \mathrm{z}$ calcd. for $\mathrm{C}_{28} \mathrm{H}_{42} \mathrm{O}_{2} \mathrm{Na}^{+}[\mathrm{M}+\mathrm{Na}]^{+}:$433.3077, found: 433.3099 .

Opt. act. $[\mathrm{a}]_{\mathrm{D}}^{25}=+54.9\left(c=1.00, \mathrm{CHCl}_{3}\right)$.

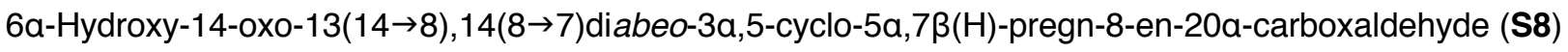

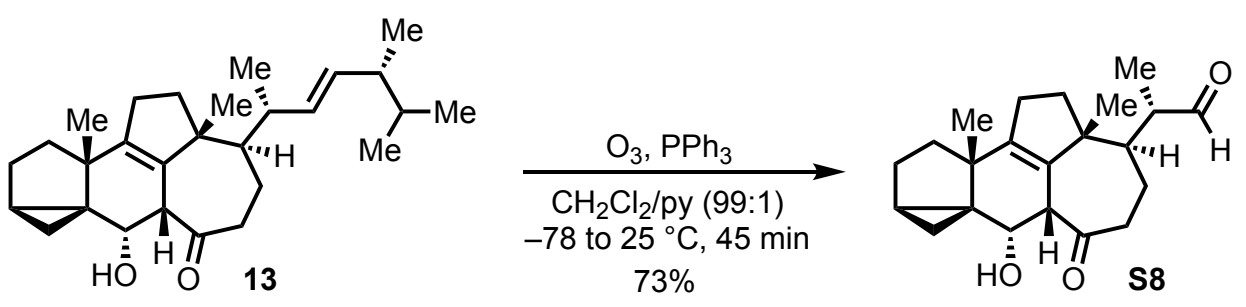


A stream of ozone-rich oxygen was passed through a solution of $\beta$-hydroxy ketone $13(574 \mathrm{mg}, 1.40 \mathrm{mmol}$, 1.0 eq.) in $\mathrm{CH}_{2} \mathrm{Cl}_{2} /$ pyridine $\left(99: 1,14 \mathrm{~mL}\right.$ ) at $-78^{\circ} \mathrm{C}$ for $45 \mathrm{~min}$. $\mathrm{PPh}_{3}$ (732 mg, $2.80 \mathrm{mmol}, 2.0$ eq.) was added and the reaction mixture was allowed to warm to $25^{\circ} \mathrm{C}$ over $16 \mathrm{~h}$. The solvent was removed, the residue was adsorbed on silica gel and column chromatography (silica gel, nhexane/EtOAc 5:1) gave aldehyde S8 (349 mg, $1.02 \mathrm{mmol}, 73 \%$ ) as a crystalline solid.

M.p. $\quad 122-124^{\circ} \mathrm{C}\left(\mathrm{CHCl}_{3}\right)$

TLC: $\quad R_{\mathrm{f}}=0.19($ nhexane/EtOAc $5: 1)$.

1H-NMR: $\quad\left(600 \mathrm{MHz}, \mathrm{CDCl}_{3}\right) ; \delta[\mathrm{ppm}]=9.77(\mathrm{~d}, J=2.4 \mathrm{~Hz}, 1 \mathrm{H}), 4.37(\mathrm{~d}, J=8.6 \mathrm{~Hz}, 1 \mathrm{H}), 4.29-4.19$ $(\mathrm{m}, 1 \mathrm{H}), 3.22(\mathrm{~d}, J=6.3 \mathrm{~Hz}, 1 \mathrm{H}), 2.76-2.66(\mathrm{~m}, 1 \mathrm{H}), 2.56-2.46(\mathrm{~m}, 2 \mathrm{H}), 2.37-2.30(\mathrm{~m}$, $1 \mathrm{H}), 2.24$ (ddt, $J=16.1,9.2,2.1 \mathrm{~Hz}, 1 \mathrm{H}$ ), 2.04 (ddd, $J=12.7,7.7,2.3 \mathrm{~Hz}, 1 \mathrm{H}), 1.82-1.77$ $(\mathrm{m}, 1 \mathrm{H}), 1.70(\mathrm{dt}, J=12.7,9.2 \mathrm{~Hz}, 1 \mathrm{H}), 1.65-1.60(\mathrm{~m}, 4 \mathrm{H}), 1.52-1.44(\mathrm{~m}, 1 \mathrm{H}), 1.17(\mathrm{~d}$, $J=7.1 \mathrm{~Hz}, 3 \mathrm{H}), 1.11(\mathrm{~s}, 3 \mathrm{H}), 1.10(\mathrm{~s}, 3 \mathrm{H}), 1.08-1.04(\mathrm{~m}, 2 \mathrm{H}), 0.98(\mathrm{dd}, J=8.4,4.9 \mathrm{~Hz}$, $1 \mathrm{H}), 0.33(\mathrm{t}, J=4.3 \mathrm{~Hz}, 1 \mathrm{H})$.

${ }^{13} \mathrm{C}-\mathrm{NMR}: \quad\left(151 \mathrm{MHz}, \mathrm{CDCl}_{3}\right) ; \delta[\mathrm{ppm}]=218.3,205.0,146.1,136.3,68.1,53.5,53.0,48.6,48.4,46.1$, $45.4,37.9,37.8,33.1,28.1,26.6,22.6,21.6,21.3,20.0,15.7,7.8$.

IR: $\quad$ (neat); $\tilde{v}\left[\mathrm{~cm}^{-1}\right]=3446(\mathrm{br} \mathrm{w}), 2954(\mathrm{~s}), 2925(\mathrm{~s}), 2857(\mathrm{~m}), 1718(\mathrm{~m}), 1684(\mathrm{~m}), 1456(\mathrm{w})$, $1418(w), 1378(w), 1281(w), 1069(w), 1029(w)$.

HRMS: (ESI-TOF); $\mathrm{m} / \mathrm{z}$ calcd. for $\mathrm{C}_{22} \mathrm{H}_{30} \mathrm{O}_{3} \mathrm{Na}^{+}[\mathrm{M}+\mathrm{Na}]^{+}:$365.2087, found: 365.2104.

Opt. act. $[\mathrm{a}]_{\mathrm{D}}^{28}=+56.0\left(c=1.00, \mathrm{CHCl}_{3}\right)$.

(22Z)-6a-Hydroxy-13(14 $\rightarrow 8), 14(8 \rightarrow 7)$ diabeo-3a,5-cyclo-5a,7ß(H)-campesta-8,22-dien-14-one (14)
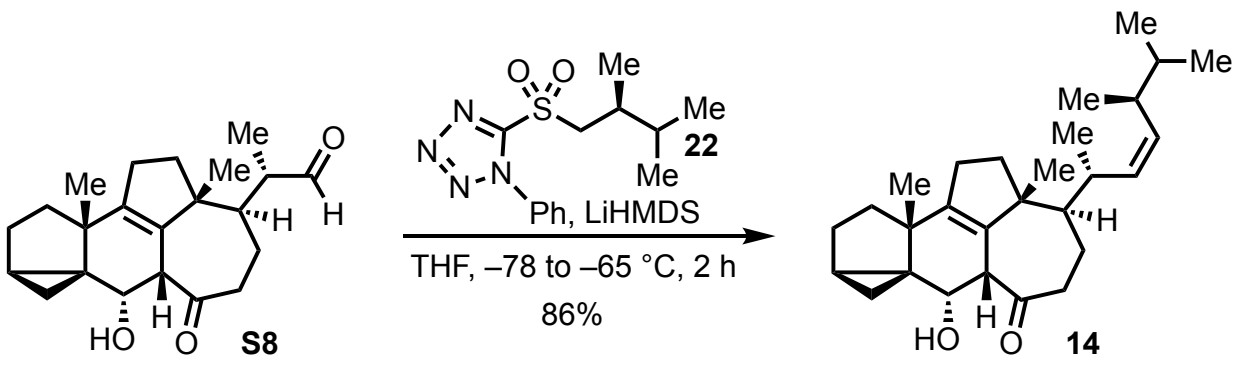

To a solution of hexamethyldisilazane (520 $\mu \mathrm{L}, 2.52 \mathrm{mmol}, 3.26 \mathrm{eq}$.) in THF (1 mL) at $0{ }^{\circ} \mathrm{C}$ was added $n \mathrm{BuLi}$ (1.6 $\mathrm{M}$ in nhexane, $1.5 \mathrm{~mL}, 2.40 \mathrm{mmol}, 3.1 \mathrm{eq}$ ) and it was stirred for $15 \mathrm{~min}$ at this temperature before the solution was added to sulfone $22\left(1.14 \mathrm{~g}, 3.87 \mathrm{mmol}, 5.0\right.$ eq.) in THF $(3 \mathrm{~mL})$ at $-78^{\circ} \mathrm{C}$. The resulting bright yellow solution was stirred at $-65^{\circ} \mathrm{C}$ for $1 \mathrm{~h}$ and a solution of aldehyde S8 (265 mg, $774 \mu \mathrm{mol}, 1.0 \mathrm{eq}$.) in THF (2 mL) was added to the reaction mixture over $30 \mathrm{~min}$. The reaction was stirred at $-65^{\circ} \mathrm{C}$ for further 30 min before EtOAc $(3 \mathrm{~mL})$ and $\mathrm{H}_{2} \mathrm{O}(5 \mathrm{~mL})$ were added to the reaction mixture. The aqueous phase was 
extracted with EtOAc $(3 \times 10 \mathrm{~mL})$ and the combined organic phases were washed sequentially with $\mathrm{HCl}$ (1 $\mathrm{M}$ in $\mathrm{H}_{2} \mathrm{O}, 20 \mathrm{~mL}$ ), $\mathrm{NaHCO}_{3}$ (sat. aq., $20 \mathrm{~mL}$ ) and brine (sat., $20 \mathrm{~mL}$ ), dried over $\mathrm{MgSO}_{4}$, and the solvent was removed under reduced pressure. Column chromatography (silica gel, nhexane/EtOAc 19:1) gave (22Z)-olefin 14 (273 mg, $665 \mu \mathrm{mol}, 86 \%$ ) as a crystalline solid and aldol product S9 (11 mg, $23 \mu \mathrm{mol}, 3 \%$ ) as a colorless solid and re-isolated sulfone $22(855 \mathrm{mg}, 2.90 \mathrm{mmol}, 3.75 \mathrm{eq}$.) as a colorless oil.

M.p. $\quad 88-90{ }^{\circ} \mathrm{C}\left(\mathrm{CHCl}_{3}\right)$

TLC: $\quad R_{\mathrm{f}}=0.33($ hhexane/EtOAc 9:1).

1H-NMR: $\quad\left(600 \mathrm{MHz}, \mathrm{CDCl}_{3}\right) ; \delta[\mathrm{ppm}]=5.25(\mathrm{t}, J=10.7 \mathrm{~Hz}, 1 \mathrm{H}), 5.06(\mathrm{t}, J=10.8 \mathrm{~Hz}, 1 \mathrm{H}), 4.42-$ $4.35(\mathrm{~m}, 1 \mathrm{H}), 4.25-4.17(\mathrm{~m}, 1 \mathrm{H}), 3.26-3.18(\mathrm{~m}, 1 \mathrm{H}), 2.83-2.72(\mathrm{~m}, 1 \mathrm{H}), 2.57-2.51$ $(\mathrm{m}, 1 \mathrm{H}), 2.50-2.45(\mathrm{~m}, 1 \mathrm{H}), 2.34-2.27(\mathrm{~m}, 1 \mathrm{H}), 2.24-2.15(\mathrm{~m}, 2 \mathrm{H}), 2.12$ (ddd, $J=12.4$, $7.8,2.6 \mathrm{~Hz}, 1 \mathrm{H}), 1.88-1.82(\mathrm{~m}, 1 \mathrm{H}), 1.66-1.59(\mathrm{~m}, 3 \mathrm{H}), 1.55-1.47(\mathrm{~m}, 2 \mathrm{H}), 1.46-1.42$ (m, $1 \mathrm{H}), 1.40-1.35(\mathrm{~m}, 1 \mathrm{H}), 1.12-1.07(\mathrm{~m}, 4 \mathrm{H}), 1.07-1.03(\mathrm{~m}, 1 \mathrm{H}), 1.02(\mathrm{~d}, J=7.0 \mathrm{~Hz}$, $3 \mathrm{H}), 0.97(\mathrm{~d}, J=4.9 \mathrm{~Hz}, 1 \mathrm{H}), 0.96(\mathrm{~s}, 3 \mathrm{H}), 0.91(\mathrm{~d}, J=6.7 \mathrm{~Hz}, 3 \mathrm{H}), 0.89(\mathrm{~d}, J=6.7 \mathrm{~Hz}$, $3 \mathrm{H}), 0.85(\mathrm{~d}, J=6.8 \mathrm{~Hz}, 3 \mathrm{H}), 0.31(\mathrm{t}, J=4.3 \mathrm{~Hz}, 1 \mathrm{H})$.

13C-NMR: $\quad\left(151 \mathrm{MHz}, \mathrm{CDCl}_{3}\right) ; \delta[\mathrm{ppm}]=219.2,144.9,137.6,133.5,131.8,68.2,54.2,53.3,48.5$, $46.2,45.3,38.4,38.1,37.8,33.6,33.4,33.1,28.2,26.5,23.0,22.7,21.0,20.3,20.3,20.1$, 20.0, 17.7, 7.7.

IR: $\quad\left(\right.$ neat); $\tilde{v}\left[\mathrm{~cm}^{-1}\right]=2952(\mathrm{~m}), 2924(\mathrm{~s}), 2854(\mathrm{~m}), 1740(\mathrm{w}), 1685(\mathrm{w}), 1458(\mathrm{w}), 1377(\mathrm{w})$.

HRMS: $\quad$ (ESI-TOF); $m / z$ calcd. for $\mathrm{C}_{28} \mathrm{H}_{42} \mathrm{O}_{2} \mathrm{Na}^{+}[\mathrm{M}+\mathrm{Na}]+:$ 433.3077, found: 433.3085 .

Opt. act. $[\mathrm{a}]_{\mathrm{D}}^{28}=+28.5\left(c=1.00, \mathrm{CHCl}_{3}\right)$.

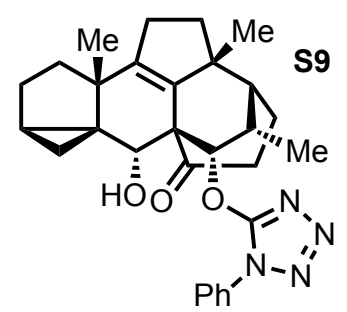

M.p. $\quad 190-193^{\circ} \mathrm{C}\left(\mathrm{CHCl}_{3}\right)$

TLC: $\quad R_{\mathrm{f}}=0.33($ nhexane/EtOAc 3:1).

1H-NMR: $\quad\left(600 \mathrm{MHz}, \mathrm{CDCl}_{3}\right) ; \delta[\mathrm{ppm}]=7.82(\mathrm{~d}, J=8.3 \mathrm{~Hz}, 2 \mathrm{H}), 7.57-7.51(\mathrm{~m}, 2 \mathrm{H}), 7.44-7.40(\mathrm{~m}$, $1 \mathrm{H}), 5.70(\mathrm{~d}, J=9.7 \mathrm{~Hz}, 1 \mathrm{H}), 4.30(\mathrm{~d}, J=9.3 \mathrm{~Hz}, 1 \mathrm{H}), 4.15(\mathrm{~d}, J=9.3 \mathrm{~Hz}, 1 \mathrm{H}), 3.32-3.24$ (m, $1 \mathrm{H}$ ), 3.15 (dt, $J=13.5,10.9 \mathrm{~Hz}, 1 \mathrm{H}$ ), 2.61 (ddd, $J=16.1,10.5,5.8 \mathrm{~Hz}, 1 \mathrm{H}$ ), $2.30-2.20$ $(\mathrm{m}, 2 \mathrm{H}), 2.15-2.07(\mathrm{~m}, 2 \mathrm{H}), 1.98-1.86(\mathrm{~m}, 2 \mathrm{H}), 1.79-1.71(\mathrm{~m}, 2 \mathrm{H}), 1.65-1.57(\mathrm{~m}$, $2 \mathrm{H}), 1.39-1.32(\mathrm{~m}, 1 \mathrm{H}), 1.26(\mathrm{~s}, 3 \mathrm{H}), 1.09-1.05(\mathrm{~m}, 1 \mathrm{H}), 1.04(\mathrm{~s}, 3 \mathrm{H}), 1.02-0.98(\mathrm{~m}$, $1 \mathrm{H}), 0.87(\mathrm{~d}, J=7.5 \mathrm{~Hz}, 3 \mathrm{H}), 0.22(\mathrm{t}, J=4.5 \mathrm{~Hz}, 1 \mathrm{H}$ ). 
13C-NMR: $\quad\left(151 \mathrm{MHz}, \mathrm{CDCl}_{3}\right) ; \delta[\mathrm{ppm}]=218.6,160.4,145.1,140.8,133.8,129.7,128.8,121.2,83.2$, $72.1,57.0,48.8,45.5,43.9,40.2,38.1,37.8,33.0,32.0,29.2,26.9,24.1,23.6,20.6,18.6$, 14.7, 7.0.

IR: $\quad\left(\right.$ neat); $\tilde{v}\left[\mathrm{~cm}^{-1}\right]=2954(\mathrm{~m}), 2923(\mathrm{~s}), 2853(\mathrm{~m}), 1740(\mathrm{w}), 1558(\mathrm{w}), 1459(\mathrm{w}), 1377(\mathrm{w})$.

HRMS: $\quad$ (ESI-TOF); $m / z$ calcd. $\mathrm{C}_{29} \mathrm{H}_{34} \mathrm{~N}_{4} \mathrm{O}_{3} \mathrm{Na}^{+}[\mathrm{M}+\mathrm{Na}]^{+}: 509.2523$, found: 509.2518.

Opt. act. $[\mathrm{a}]_{\mathrm{D}}^{20}=-41.6\left(\mathrm{c}=1.00, \mathrm{CHCl}_{3}\right)$.

13(14 $\rightarrow 8), 14(8 \rightarrow 7)$ diabeo-3a,5-cyclo-5a,7ß(H)-Campesta-8-en-6a,14a,23-triol (S10)
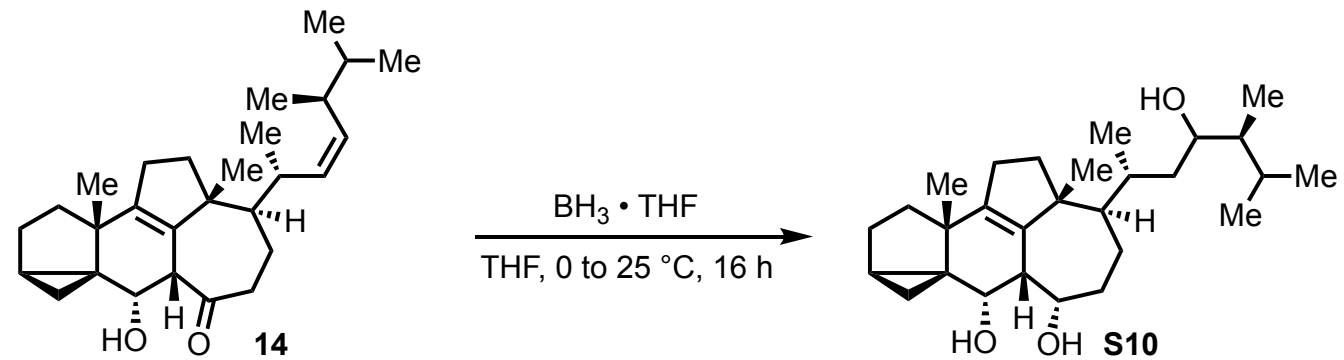

To a solution of (22Z)-olefin 14 (339 mg, $825 \mu \mathrm{mol}, 1.0$ eq.) in THF (8.3 mL) was added $\mathrm{BH}_{3} \cdot \mathrm{THF}(1 \mathrm{M}$ in THF, $8.3 \mathrm{~mL}, 8.3 \mathrm{mmol}, 10$ eq.) at $0{ }^{\circ} \mathrm{C}$ and the reaction mixture was allowed to warm to $25^{\circ} \mathrm{C}$ over $16 \mathrm{~h}$. It was diluted with $\mathrm{Et}_{2} \mathrm{O}(10 \mathrm{~mL})$ at $0{ }^{\circ} \mathrm{C}, \mathrm{NaOH}\left(10 \%\right.$ w/w in $\left.\mathrm{H}_{2} \mathrm{O}, 5 \mathrm{~mL}\right)$ and $\mathrm{H}_{2} \mathrm{O}_{2}\left(35 \%\right.$ w/w in $\left.\mathrm{H}_{2} \mathrm{O}, 5 \mathrm{~mL}\right)$

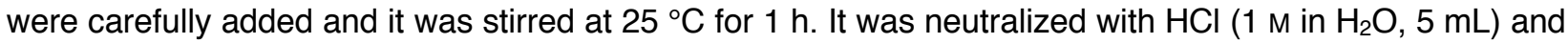
the aqueous phase was extracted with $\mathrm{Et}_{2} \mathrm{O}(3 \times 15 \mathrm{~mL})$. The combined organic phases were washed sequentially with $\mathrm{NaHCO}_{3}$ (sat. aq., $30 \mathrm{~mL}$ ) and brine (sat., $30 \mathrm{~mL}$ ), dried over $\mathrm{MgSO}_{4}$, and the solvent was removed under reduced pressure. The crude mixture of regio- and diastereomers (303 mg, $704 \mu \mathrm{mol}$, combined yield $85 \%$ ) was used in the next step without further purification.

Analytically pure triol $\mathbf{S} 10$ (major product, $\mathrm{C} 23-\mathrm{OH}$, configuration at $\mathrm{C} 23$ was not elucidated) could be obtained by column chromatography (silica gel, nhexane/EtOAc $5: 1 \rightarrow 3: 1$ ) as a colorless foam.

TLC: $\quad R_{\mathrm{f}}=0.24($ nhexane/EtOAc $3: 1)$.

1H-NMR: $\quad\left(600 \mathrm{MHz}, \mathrm{CDCl}_{3}\right) ; \delta[\mathrm{ppm}]=4.27(\mathrm{~d}, J=6.3 \mathrm{~Hz}, 1 \mathrm{H}), 4.23(\mathrm{~d}, J=6.2 \mathrm{~Hz}, 1 \mathrm{H}), 3.52$ (ddd, $J=10.6,7.0,1.6 \mathrm{~Hz}, 1 \mathrm{H}), 2.68(\mathrm{~d}, J=6.3 \mathrm{~Hz}, 1 \mathrm{H}), 2.29-2.18(\mathrm{~m}, 2 \mathrm{H}), 2.01-1.94(\mathrm{~m}$, $2 \mathrm{H}), 1.94-1.87(\mathrm{~m}, 1 \mathrm{H}), 1.87-1.81(\mathrm{~m}, 1 \mathrm{H}), 1.79-1.74(\mathrm{~m}, 1 \mathrm{H}), 1.73-1.66(\mathrm{~m}, 1 \mathrm{H})$, $1.65-1.57(\mathrm{~m}, 4 \mathrm{H}), 1.57-1.48(\mathrm{~m}, 3 \mathrm{H}), 1.47-1.39(\mathrm{~m}, 2 \mathrm{H}), 1.35-1.29(\mathrm{~m}, 1 \mathrm{H}), 1.10-$ $1.07(\mathrm{~m}, 1 \mathrm{H}), 1.04(\mathrm{~s}, 3 \mathrm{H}), 1.03(\mathrm{~s}, 3 \mathrm{H}), 0.93-0.89(\mathrm{~m}, 6 \mathrm{H}), 0.83(\mathrm{~d}, J=6.8 \mathrm{~Hz}, 3 \mathrm{H}), 0.76$ $(\mathrm{d}, J=6.9 \mathrm{~Hz}, 3 \mathrm{H}), 0.69$ (dd, $J=8.4,5.0 \mathrm{~Hz}, 1 \mathrm{H}), 0.28(\mathrm{t}, J=4.5 \mathrm{~Hz}, 1 \mathrm{H})$. 
${ }^{13} \mathrm{C}-\mathrm{NMR}: \quad\left(151 \mathrm{MHz}, \mathrm{CDCl}_{3}\right) ; \delta[\mathrm{ppm}]=144.7,140.1,72.4,66.4,64.2,53.8,51.8,45.7,45.5,42.5$, $39.5,37.4,36.3,35.8,33.2,30.1,28.5,28.2,26.1,23.9,21.7,21.6,20.8,20.5,18.5,17.8$, 10.5, 7.1.

IR: $\quad\left(\right.$ neat); $\tilde{\text { v }}\left[\mathrm{cm}^{-1}\right]=3320(\mathrm{br} \mathrm{w}), 2953(\mathrm{~s}), 2925(\mathrm{w}), 2870(\mathrm{~m}), 1740(\mathrm{~m}), 1463(\mathrm{~m}), 1367(\mathrm{~m})$, $1217(\mathrm{w}), 1045(\mathrm{~m}), 754(\mathrm{w})$.

HRMS: $\quad$ (ESI-TOF); $\mathrm{m} / z$ calcd. for $\mathrm{C}_{28} \mathrm{H}_{46} \mathrm{O}_{3} \mathrm{Na}^{+}[\mathrm{M}+\mathrm{Na}]^{+}:$453.3339, found: 453.3318 .

Opt. act. $\quad[a]_{D}^{19}=+101.3\left(c=1.00, \mathrm{CHCl}_{3}\right)$.

6,14-(1-Methylethylidene acetal)-13(14 $\rightarrow 8), 14(8 \rightarrow 7)$ diabeo-3a,5-cyclo-5a,7 $\beta(\mathrm{H})$-campesta-8-en6a,14a,23-triol (S11)
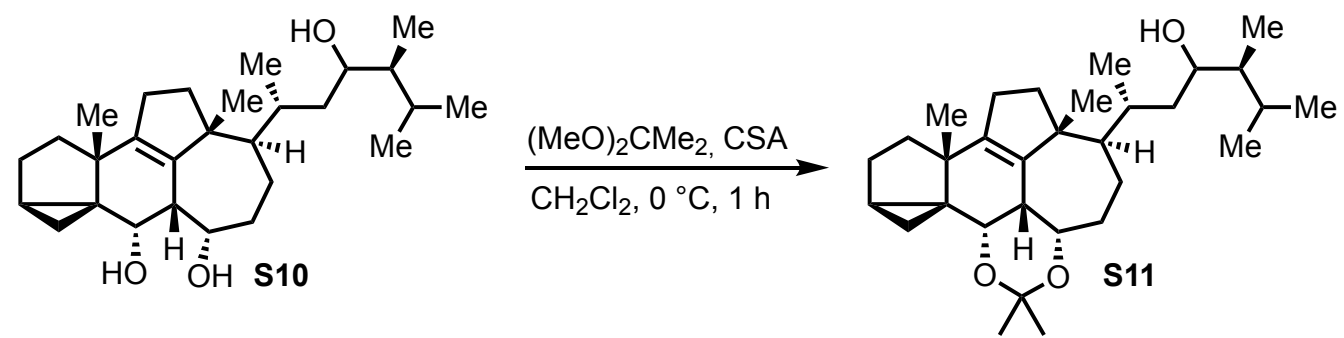

To a solution of the crude mix of regio- and diastereomers $\mathbf{S} 10$ (296 mg, $687 \mu \mathrm{mol}, 1.0 \mathrm{eq}$ ) in $\mathrm{CH}_{2} \mathrm{Cl}_{2} / 2,2-$ dimethoxy propane $(5: 1,6.6 \mathrm{~mL}$ ) was added camphorsulfonic acid (192 mg, $825 \mu \mathrm{mol}, 1.2 \mathrm{eq}$ ) $)$ at $0^{\circ} \mathrm{C}$. The reaction mixture was stirred at this temperature for $1 \mathrm{~h}$ before $\mathrm{NaHCO}_{3}$ (sat. aq., $5 \mathrm{~mL}$ ) was added. The aqueous phase was extracted with $\mathrm{CH}_{2} \mathrm{Cl}_{2}(3 \times 10 \mathrm{~mL})$ and the combined organic phases were washed with brine (sat., $30 \mathrm{~mL}$ ), dried over $\mathrm{MgSO}_{4}$ and the solvent was removed under reduced pressure. The crude product was used without further purification in the next step.

Analytically pure C23-hydroxy epimers $\mathbf{S 1 1 a}$ and $\mathbf{S 1 1}$ b could be obtained by column chromatography (silica gel, nhexane/EtOAc 19:1 $\rightarrow$ 9:1) as colorless oils.

\section{S11a}

TLC: $\quad R_{\mathrm{f}}=0.20($ nhexane/EtOAc $9: 1)$.

1H-NMR: $\quad\left(500 \mathrm{MHz}, \mathrm{CDCl}_{3}\right) ; \delta[\mathrm{ppm}]=4.48(\mathrm{~d}, J=7.2 \mathrm{~Hz}, 1 \mathrm{H}), 3.95(\mathrm{ddd}, J=11.5,9.5,2.0 \mathrm{~Hz}, 1 \mathrm{H})$, 3.54 (dd, $J=10.5,7.0 \mathrm{~Hz}, 1 \mathrm{H}$ ), 3.04 (ddt, $J=9.6,7.3,2.3 \mathrm{~Hz}, 1 \mathrm{H}), 2.27-2.18(\mathrm{~m}, 1 \mathrm{H})$, $2.11(\mathrm{dd}, J=12.2,6.6 \mathrm{~Hz}, 1 \mathrm{H}), 2.09-2.04(\mathrm{~m}, 1 \mathrm{H}), 2.00$ (ddd, $J=15.5,8.8,2.3 \mathrm{~Hz}, 1 \mathrm{H}$ ), $1.86-1.78(\mathrm{~m}, 1 \mathrm{H}), 1.71-1.65(\mathrm{~m}, 2 \mathrm{H}), 1.60-1.51(\mathrm{~m}, 5 \mathrm{H}), 1.49(\mathrm{~s}, 3 \mathrm{H}), 1.39-1.33(\mathrm{~m}$, $2 \mathrm{H}), 1.32-1.27(\mathrm{~m}, 2 \mathrm{H}), 1.24(\mathrm{~s}, 3 \mathrm{H}), 1.22-1.18(\mathrm{~m}, 1 \mathrm{H}), 1.14(\mathrm{dd}, J=12.9,2.7 \mathrm{~Hz}, 1 \mathrm{H})$, $1.10-1.05(\mathrm{~m}, 1 \mathrm{H}), 1.03(\mathrm{~s}, 3 \mathrm{H}), 0.98(\mathrm{~s}, 3 \mathrm{H}), 0.95(\mathrm{~d}, J=6.9 \mathrm{~Hz}, 3 \mathrm{H}), 0.90(\mathrm{~d}, J=6.8 \mathrm{~Hz}$, 
3H), $0.83(\mathrm{~d}, J=6.8 \mathrm{~Hz}, 3 \mathrm{H}), 0.79(\mathrm{dd}, J=8.0,4.6 \mathrm{~Hz}, 1 \mathrm{H}), 0.75(\mathrm{~d}, J=6.9 \mathrm{~Hz}, 3 \mathrm{H}), 0.27$ (t, $J=4.1 \mathrm{~Hz}, 1 \mathrm{H})$.

${ }^{13} \mathrm{C}-N M R: \quad\left(126 \mathrm{MHz}, \mathrm{CDCl}_{3}\right) ; \delta[\mathrm{ppm}]=142.9,140.3,96.8,72.4,72.0,68.7,54.1,49.8,45.9,44.6$, $38.0,36.9,36.5,33.4,33.1,32.9,30.3,30.1$, 28.2, 27.9, 26.0, 25.7, 23.8, 22.2, 21.6, 21.3, $21.2,20.5,17.9,10.5,6.6$.

IR: $\quad\left(\right.$ neat); $\tilde{v}\left[\mathrm{~cm}^{-1}\right]=2953(\mathrm{~m}), 2923(\mathrm{~s}), 2854(\mathrm{~m}), 1739(\mathrm{w}), 1461(\mathrm{~m}), 1378(\mathrm{~m}), 1244(\mathrm{w})$, $1212(w), 1094(w), 1050(w), 873(w)$.

HRMS: $\quad$ (ESI-TOF); $\mathrm{m} / z$ calcd. for $\mathrm{C}_{31} \mathrm{H}_{50} \mathrm{O}_{3} \mathrm{Na}+[\mathrm{M}+\mathrm{Na}]+:$ 493.3652, found: 493.3630.

Opt. act. $\quad[\mathrm{a}]_{\mathrm{D}}^{22}=+74.3\left(c=1.00, \mathrm{CHCl}_{3}\right)$.

\section{S11b}

TLC: $\quad R_{\mathrm{f}}=0.23($ nhexane/EtOAc $5: 1)$.

1H-NMR: $\quad\left(600 \mathrm{MHz}, \mathrm{CDCl}_{3}\right) ; \delta[\mathrm{ppm}]=4.49(\mathrm{~d}, J=7.2 \mathrm{~Hz}, 1 \mathrm{H}), 3.97-3.91(\mathrm{~m}, 1 \mathrm{H}), 3.82-3.76(\mathrm{~m}$, $1 \mathrm{H}), 3.04(\mathrm{dt}, J=9.7,5.0 \mathrm{~Hz}, 1 \mathrm{H}), 2.26-2.18(\mathrm{~m}, 1 \mathrm{H}), 2.09-1.97(\mathrm{~m}, 2 \mathrm{H}), 1.77-1.66$ $(\mathrm{m}, 3 \mathrm{H}), 1.65-1.53(\mathrm{~m}, 5 \mathrm{H}), 1.50(\mathrm{~s}, 3 \mathrm{H}), 1.36-1.29(\mathrm{~m}, 1 \mathrm{H}), 1.28-1.25(\mathrm{~m}, 1 \mathrm{H}), 1.24$ (s, 3H), $1.23-1.17(\mathrm{~m}, 1 \mathrm{H}), 1.14-1.06(\mathrm{~m}, 3 \mathrm{H}), 1.04(\mathrm{~s}, 3 \mathrm{H}), 1.03(\mathrm{~d}, J=7.6 \mathrm{~Hz}, 3 \mathrm{H})$, $1.00-0.97(\mathrm{~m}, 1 \mathrm{H}), 0.96(\mathrm{~d}, J=6.7 \mathrm{~Hz}, 3 \mathrm{H}), 0.94-0.90(\mathrm{~m}, 6 \mathrm{H}), 0.88(\mathrm{~d}, J=6.8 \mathrm{~Hz}, 1 \mathrm{H})$, $0.85(\mathrm{~d}, J=6.9 \mathrm{~Hz}, 3 \mathrm{H}), 0.80(\mathrm{dd}, J=8.2,4.5 \mathrm{~Hz}, 1 \mathrm{H}), 0.28(\mathrm{t}, J=4.1 \mathrm{~Hz}, 1 \mathrm{H})$.

13C-NMR: $\quad\left(151 \mathrm{MHz}, \mathrm{CDCl}_{3}\right) ; \delta[\mathrm{ppm}]=143.0,140.3,96.8,72.9,72.3,68.7,53.7,50.7,44.6,43.2$, $38.8,38.0,36.5,33.5,33.1,33.0,32.5,30.7,30.2,27.9,26.0,25.7,23.9,22.3,22.2,21.3$, 21.3, 20.4, 20.4, 9.9, 6.7.

IR: $\quad\left(\right.$ neat); $\tilde{v}\left[\mathrm{~cm}^{-1}\right]=2952(\mathrm{~m}), 2922(\mathrm{~s}), 2853(\mathrm{~m}), 1740(\mathrm{w}), 1460(\mathrm{~m}), 1377(\mathrm{w}), 1244(\mathrm{w})$, $1171(w)$.

HRMS: (ESI-TOF); $\mathrm{m} / \mathrm{z}$ calcd. for $\mathrm{C}_{31} \mathrm{H}_{50} \mathrm{O}_{3} \mathrm{Na}^{+}[\mathrm{M}+\mathrm{Na}]^{+}:$493.3652, found: 493.3646.

Opt. act. $[\mathrm{a}]_{\mathrm{D}}^{22}=+58.4\left(c=1.00, \mathrm{CHCl}_{3}\right)$.

23-(S-Methyl carbonodithioate)-6,14-(1-methylethylidene acetal)-13(14 $\rightarrow 8), 14(8 \rightarrow 7)$ diabeo-3a,5-cyclo$5 a, 7 \beta(H)$-campesta-8-en-6a,14a,23-triol (S12)

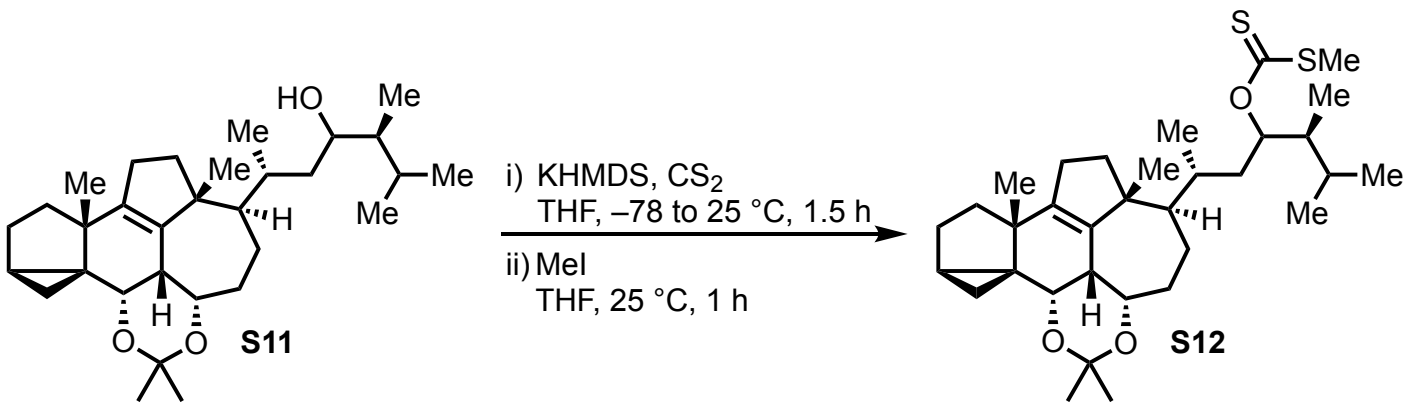


A solution of crude acetonides $\mathbf{S 1 1}$ (687 $\mu \mathrm{mol}, 1.0$ eq.) in THF $(6.9 \mathrm{~mL})$ was cooled to $78{ }^{\circ} \mathrm{C}$ and $\mathrm{KHMDS}$ ( $0.5 \mathrm{M}$ in toluene, $2.75 \mathrm{~mL}, 1.37 \mathrm{mmol}, 2.0$ eq.) and $\mathrm{CS}_{2}$ (208 $\mu \mathrm{L}, 3.44 \mathrm{mmol}, 5$ eq.) were added dropwise. After stirring at this temperature for $20 \mathrm{~min}$, the mixture was warmed to $25^{\circ} \mathrm{C}$ and stirred for $1 \mathrm{~h}$. Mel (321 $\mu \mathrm{L}, 5.15 \mathrm{mmol}, 7.5$ eq.) was added and stirring was continued at $25^{\circ} \mathrm{C}$ for 45 min before it was diluted with $\mathrm{H}_{2} \mathrm{O}(5 \mathrm{~mL})$ and $\mathrm{Et}_{2} \mathrm{O}(5 \mathrm{~mL})$. The aqueous phase was extracted with $\mathrm{Et}_{2} \mathrm{O}(3 \times 15 \mathrm{~mL})$ and the combined organic phases were washed with brine (sat., $40 \mathrm{~mL}$ ), dried over $\mathrm{MgSO}_{4}$, and the solvent was removed under reduced pressure. A mixture of xanthates S12 was obtained by filtering through a plug of silica with nhexane/EtOAc (9:1).

Analytically pure xanthate C23-epimers $\mathbf{S 1 2 a}$ and $\mathbf{S 1 2 b}$ could be obtained by column chromatography (silica gel, nhexane/EtOAc 50:1) as colorless oils.

\section{S12a}

TLC: $\quad R_{\mathrm{f}}=0.35($ nhexane/EtOAc 19:1).

1H-NMR: $\quad\left(600 \mathrm{MHz}, \mathrm{CDCl}_{3}\right) ; \delta[\mathrm{ppm}]=5.98(\mathrm{ddd}, J=11.4,5.3,1.5 \mathrm{~Hz}, 1 \mathrm{H}), 4.49(\mathrm{~d}, J=7.2 \mathrm{~Hz}, 1 \mathrm{H})$, $4.00-3.95(\mathrm{~m}, 1 \mathrm{H}), 3.05(\mathrm{ddt}, J=9.6,7.5,2.3 \mathrm{~Hz}, 1 \mathrm{H}), 2.56(\mathrm{~s}, 3 \mathrm{H}), 2.23-2.15(\mathrm{~m}, 1 \mathrm{H})$, $2.03-1.95(\mathrm{~m}, 2 \mathrm{H}), 1.88-1.82(\mathrm{~m}, 1 \mathrm{H}), 1.81-1.75(\mathrm{~m}, 1 \mathrm{H}), 1.74-1.67(\mathrm{~m}, 3 \mathrm{H}), 1.60-$ $1.52(\mathrm{~m}, 8 \mathrm{H}), 1.51(\mathrm{~s}, 3 \mathrm{H}), 1.35-1.28(\mathrm{~m}, 1 \mathrm{H}), 1.25(\mathrm{~s}, 3 \mathrm{H}), 1.15-1.10(\mathrm{~m}, 2 \mathrm{H}), 1.03(\mathrm{~s}$, $3 \mathrm{H}), 1.00(\mathrm{~d}, J=6.9 \mathrm{~Hz}, 3 \mathrm{H}), 0.98(\mathrm{~s}, 3 \mathrm{H}), 0.96(\mathrm{~d}, J=6.7 \mathrm{~Hz}, 3 \mathrm{H}), 0.89(\mathrm{~d}, J=6.7 \mathrm{~Hz}$, $3 \mathrm{H}), 0.87(\mathrm{~d}, J=7.0 \mathrm{~Hz}, 3 \mathrm{H}), 0.82-0.78(\mathrm{~m}, 1 \mathrm{H}), 0.28(\mathrm{t}, J=4.0 \mathrm{~Hz}, 1 \mathrm{H})$.

13C-NMR: $\quad\left(151 \mathrm{MHz}, \mathrm{CDCl}_{3}\right) ; \delta[\mathrm{ppm}]=216.3,143.0,140.2,96.8,85.8,72.4,68.7,53.8,49.7,44.6$, 42.8, 37.9, 36.5, 33.4, 33.2, 33.1, 32.9, 30.2, 29.9, 29.7, 27.9, 26.0, 25.7, 23.5, 22.2, 21.6, 21.3, 21.2, 20.3, 19.8, 19.0, 11.5, 6.7.

IR: $\quad\left(\right.$ neat); $\tilde{v}\left[\mathrm{~cm}^{-1}\right]=2953(\mathrm{~m}), 2923(\mathrm{~s}), 2854(\mathrm{~m}), 1738(\mathrm{w}), 1460(\mathrm{w}), 1378(\mathrm{w}), 1219(\mathrm{~s})$, $1172(w), 1095(w), 1049(s), 874(w)$.

HRMS: (ESI-TOF); $\mathrm{m} / \mathrm{z}$ calcd. for $\mathrm{C}_{33} \mathrm{H}_{52} \mathrm{O}_{3} \mathrm{~S}_{2} \mathrm{Na}^{+}[\mathrm{M}+\mathrm{Na}]^{+}: 583.3250$, found: 583.3252.

Opt. act. $\quad[\mathrm{a}]_{\mathrm{D}}^{23}=+57.5\left(c=0.44, \mathrm{CHCl}_{3}\right)$.

\section{S12b}

TLC: $\quad R_{\mathrm{f}}=0.27($ nhexane/EtOAc 19:1).

1H-NMR: $\quad\left(600 \mathrm{MHz}, \mathrm{CDCl}_{3}\right) ; \delta[\mathrm{ppm}]=5.94(\mathrm{ddd}, J=9.3,5.2,1.9 \mathrm{~Hz}, 1 \mathrm{H}), 4.49(\mathrm{~d}, J=7.2 \mathrm{~Hz}, 1 \mathrm{H})$, 3.94 (ddd, $J=11.2,9.5,2.2 \mathrm{~Hz}, 1 \mathrm{H}$ ), 3.03 (ddt, $J=9.5,7.3,2.3 \mathrm{~Hz}, 1 \mathrm{H}$ ), 2.54 (s, 3H), 2.26 $-2.17(\mathrm{~m}, 1 \mathrm{H}), 2.09-1.97(\mathrm{~m}, 2 \mathrm{H}), 1.77-1.66(\mathrm{~m}, 4 \mathrm{H}), 1.61-1.53(\mathrm{~m}, 10 \mathrm{H}), 1.53-1.50$ (m, 1H), 1.49 (s, 3H), 1.43 (ddd, J=13.5, 12.0, $5.2 \mathrm{~Hz}, 1 \mathrm{H}), 1.38-1.31(\mathrm{~m}, 2 \mathrm{H}), 1.26$ (d, $J=3.1 \mathrm{~Hz}, 1 \mathrm{H}), 1.24(\mathrm{~s}, 3 \mathrm{H}), 1.23-1.20(\mathrm{~m}, 1 \mathrm{H}), 1.11(\mathrm{dd}, J=12.7,3.0 \mathrm{~Hz}, 1 \mathrm{H}), 1.00(\mathrm{~d}$, $J=6.7 \mathrm{~Hz}, 3 \mathrm{H}$ ), $0.96(\mathrm{~s}, 3 \mathrm{H}), 0.94(\mathrm{~d}, J=6.4 \mathrm{~Hz}, 3 \mathrm{H}), 0.92(\mathrm{~d}, J=6.4 \mathrm{~Hz}, 3 \mathrm{H}), 0.90-0.85$ (m, 1H), 0.80 (dd, $J=8.3,4.5 \mathrm{~Hz}, 1 \mathrm{H}), 0.28$ (t, $J=4.1 \mathrm{~Hz}, 1 \mathrm{H}$ ). 
${ }^{13} \mathrm{C}-\mathrm{NMR}: \quad\left(151 \mathrm{MHz}, \mathrm{CDCl}_{3}\right) ; \delta[\mathrm{ppm}]=215.3,143.1,140.1,96.8,86.0,72.3,68.7,53.6,50.6,44.6$, $41.3,38.0,36.5,34.0,33.4,33.1,32.9,31.6,30.9,30.1,27.9,26.0,25.7,23.8,22.3,21.5$, 21.4, 21.1, 20.5, 20.3, 18.8, 11.6, 6.7.

IR: $\quad\left(\right.$ neat); $\tilde{v}\left[\mathrm{~cm}^{-1}\right]=2953(\mathrm{~m}), 2924(\mathrm{~s}), 2854(\mathrm{~m}), 1739(\mathrm{w}), 1459(\mathrm{w}), 1379(\mathrm{w}), 1218(\mathrm{~m})$, $1094(w), 1050(m), 874(w)$.

HRMS: (ESI-TOF); $\mathrm{m} / \mathrm{z}$ calcd. for $\mathrm{C}_{33} \mathrm{H}_{52} \mathrm{O}_{3} \mathrm{~S}_{2} \mathrm{Na}^{+}[\mathrm{M}+\mathrm{Na}]^{+}: 583.3250$, found: 583.3242.

Opt. act. $[\mathrm{a}]_{\mathrm{D}}^{24}=+53.2\left(c=0.22, \mathrm{CHCl}_{3}\right)$.

6,14-(1-Methylethylidene acetal)-13(14 $\rightarrow 8), 14(8 \rightarrow 7)$ diabeo-3a,5-cyclo-5a,7 $\beta(H)$-campesta-8-en-6a,14adiol (15)
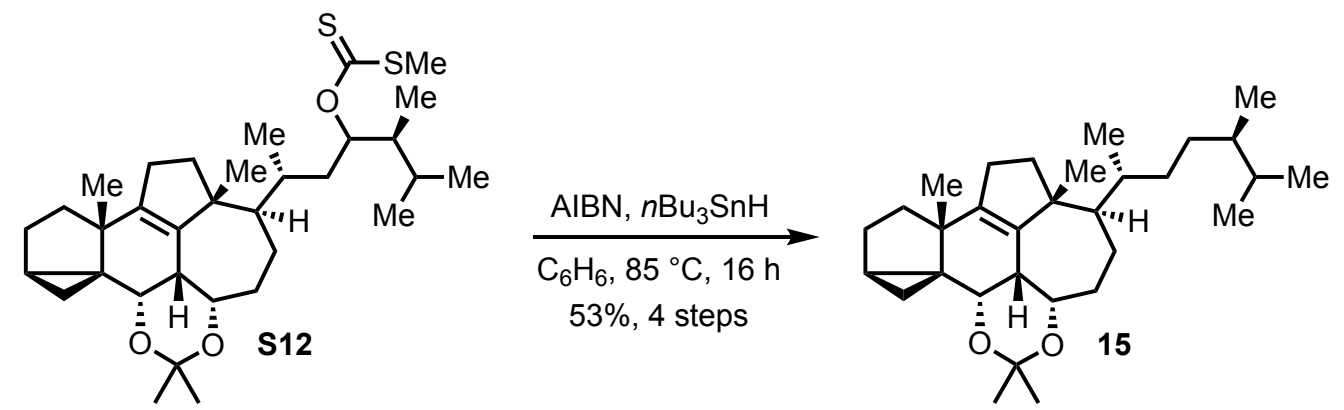

Azobisisobutyronitrile (56 mg, $344 \mu \mathrm{mol}, 0.5$ eq.) and $n \mathrm{Bu}_{3} \mathrm{SnH}$ (927 $\mu \mathrm{L}, 3.44 \mathrm{mmol}, 5$ eq.) were dissolved in benzene $(3.3 \mathrm{~mL})$ and the solution was degassed applying three freeze-pump-thaw cycles. This mixture was added to a degassed solution (also three freeze-pump-thaw cycles) of xanthates $\mathbf{S 1 2}$ (687 $\mu \mathrm{mol}$, 1.0 eq.) in benzene $(6.8 \mathrm{~mL})$ at $85^{\circ} \mathrm{C}$ over $3 \mathrm{~h}$. The reaction mixture was then allowed to cool to $25^{\circ} \mathrm{C}$ and the solvent was removed under reduced pressure. Column chromatography (silica gel, nhexane/EtOAc 50:1 $\rightarrow 19: 1 \rightarrow 12: 1)$ gave acetonide 15 (200 mg, $440 \mu$ mol, $53 \%$ over 4 steps) as a colorless oil.

TLC: $\quad R_{\mathrm{f}}=0.33($ nhexane/EtOAc 19:1).

1H-NMR: $\quad\left(400 \mathrm{MHz}, \mathrm{CDCl}_{3}\right) ; \delta[\mathrm{ppm}]=4.49(\mathrm{~d}, J=7.2 \mathrm{~Hz}, 1 \mathrm{H}), 3.96(\mathrm{ddd}, J=11.4,9.5,2.1 \mathrm{~Hz}, 1 \mathrm{H})$, 3.04 (ddt, $J=9.5,7.5,2.3 \mathrm{~Hz}, 1 \mathrm{H}), 2.26-2.16(\mathrm{~m}, 1 \mathrm{H}), 2.07-1.95(\mathrm{~m}, 2 \mathrm{H}), 1.72-1.64$ $(\mathrm{m}, 2 \mathrm{H}), 1.62-1.51(\mathrm{~m}, 6 \mathrm{H}), 1.50(\mathrm{~s}, 3 \mathrm{H}), 1.45-1.42(\mathrm{~m}, 1 \mathrm{H}), 1.37-1.26(\mathrm{~m}, 3 \mathrm{H}), 1.24$ (s, 3H), $1.23-1.13(\mathrm{~m}, 3 \mathrm{H}), 1.09$ (dd, $J=12.6,2.9 \mathrm{~Hz}, 2 \mathrm{H}), 1.03(\mathrm{~s}, 3 \mathrm{H}), 1.01-0.96(\mathrm{~m}$, $1 \mathrm{H}), 0.94-0.90(\mathrm{~m}, 6 \mathrm{H}), 0.90-0.87(\mathrm{~m}, 1 \mathrm{H}), 0.85(\mathrm{~d}, J=6.8 \mathrm{~Hz}, 3 \mathrm{H}), 0.82-0.76(\mathrm{~m}$, $6 \mathrm{H}), 0.28(\mathrm{t}, J=4.1 \mathrm{~Hz}, 1 \mathrm{H})$.

13C-NMR: $\quad\left(101 \mathrm{MHz}, \mathrm{CDCl}_{3}\right) ; \delta[\mathrm{ppm}]=142.8,140.3,96.8,72.4,68.7,53.9,50.2,44.6,39.2,38.0$, $36.5,35.3,33.5,33.4,33.1,33.0,32.2,30.9,30.5,30.2,27.9,26.0,25.7,23.7,22.3,21.3$, $20.4,20.3,18.2,15.7,6.7$. 
IR: $\quad\left(\right.$ neat); $\tilde{v}\left[\mathrm{~cm}^{-1}\right]=2989(\mathrm{w}), 2954(\mathrm{~s}), 2867(\mathrm{~m}), 2359(\mathrm{w}), 1458(\mathrm{w}), 1377(\mathrm{~m}), 1244(\mathrm{~m})$, $1212(\mathrm{~m}), 1094(\mathrm{~m}), 747(\mathrm{~m})$.

HRMS: $\quad$ (ESI-TOF); $m / z$ calcd. for $\mathrm{C}_{31} \mathrm{H}_{50} \mathrm{O}_{2} \mathrm{Na}^{+}[\mathrm{M}+\mathrm{Na}]^{+}: 477.3703$, found: 477.3699 .

Opt. act. $[\mathrm{a}]_{\mathrm{D}}^{24}=+27.8\left(c=1.00, \mathrm{CHCl}_{3}\right)$.

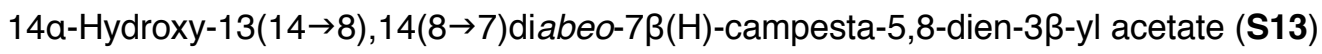

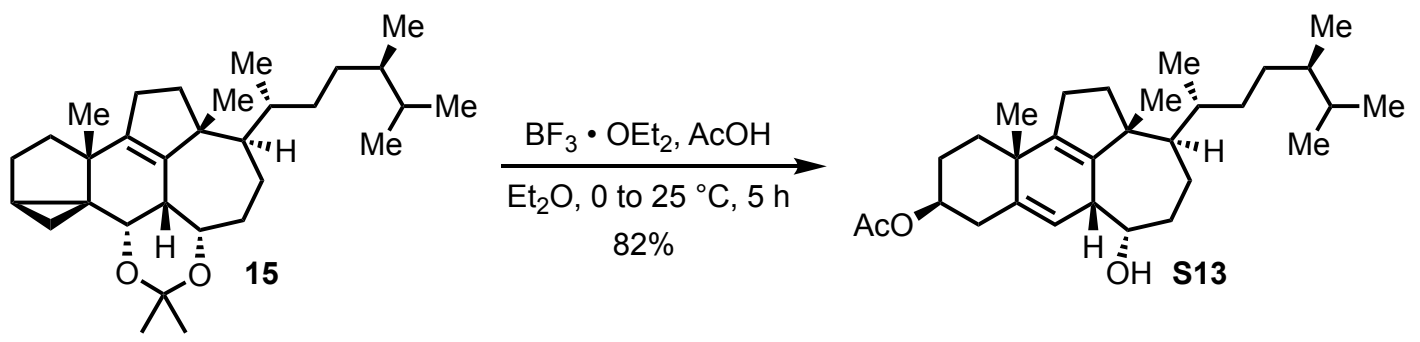

To a solution of acetonide 15 (200 mg, $440 \mu$ mol, 1.0 eq.) in $\mathrm{Et}_{2} \mathrm{O}(4.4 \mathrm{~mL})$ were added acetic acid (2.2 mL) and $\mathrm{BF}_{3} \cdot \mathrm{OEt}_{2}(2.2 \mathrm{~mL})$ at $0{ }^{\circ} \mathrm{C}$. The resulting solution was warmed to $25^{\circ} \mathrm{C}$ and stirred for $5 \mathrm{~h}$, before diluting with EtOAc $(10 \mathrm{~mL})$. The reaction mixture was then carefully poured into $\mathrm{NaHCO}_{3}$ (sat. aq., $100 \mathrm{~mL}$ ) and the aqueous phase was extracted with EtOAc $(3 \times 20 \mathrm{~mL})$. The combined organic phases were washed sequentially with $\mathrm{NaHCO}_{3}$ (sat. aq., $50 \mathrm{~mL}$ ) and brine (sat., $50 \mathrm{~mL}$ ), dried over $\mathrm{MgSO}_{4}$, and the solvent was removed under reduced pressure. Column chromatography (silica gel, nhexane/EtOAc 5:1) gave acetate S13 (165 mg, $361 \mu \mathrm{mol}, 82 \%)$ as a colorless oil.

TLC: $\quad R_{\mathrm{f}}=0.25($ nhexane/EtOAc 5:1).

1H-NMR: $\quad\left(500 \mathrm{MHz}, \mathrm{CDCl}_{3}\right) ; \delta[\mathrm{ppm}]=5.43-5.34(\mathrm{~m}, 1 \mathrm{H}), 4.64(\mathrm{tt}, J=11.4,5.0 \mathrm{~Hz}, 1 \mathrm{H}), 3.84-$ $3.76(\mathrm{~m}, 1 \mathrm{H}), 3.02-2.95(\mathrm{~m}, 1 \mathrm{H}), 2.47$ (ddd, $J=12.5,5.3,1.8 \mathrm{~Hz}, 1 \mathrm{H}), 2.44-2.36(\mathrm{~m}$, 1H), $2.35-2.28(\mathrm{~m}, 1 \mathrm{H}), 2.23$ (dddd, $J=15.8,8.7,2.7,1.4 \mathrm{~Hz}, 1 \mathrm{H}), 2.03$ (s, 3H), $1.95-$ $1.88(\mathrm{~m}, 3 \mathrm{H}), 1.82(\mathrm{ddd}, J=12.0,7.5,2.6 \mathrm{~Hz}, 1 \mathrm{H}), 1.73-1.66(\mathrm{~m}, 2 \mathrm{H}), 1.65-1.57(\mathrm{~m}$, $3 \mathrm{H}), 1.56-1.51(\mathrm{~m}, 3 \mathrm{H}), 1.40-1.32(\mathrm{~m}, 2 \mathrm{H}), 1.30-1.23(\mathrm{~m}, 2 \mathrm{H}), 1.15(\mathrm{~s}, 3 \mathrm{H}), 1.01-$ $0.97(\mathrm{~m}, 1 \mathrm{H}), 0.96(\mathrm{~s}, 3 \mathrm{H}), 0.94-0.91(\mathrm{~m}, 1 \mathrm{H}), 0.89(\mathrm{~d}, J=6.8 \mathrm{~Hz}, 3 \mathrm{H}), 0.85(\mathrm{~d}, J=6.8$ $\mathrm{Hz}, 3 \mathrm{H}), 0.79(\mathrm{~d}, J=6.8 \mathrm{~Hz}, 3 \mathrm{H}), 0.77(\mathrm{~d}, J=6.5 \mathrm{~Hz}, 3 \mathrm{H})$.

13C-NMR: $\quad\left(126 \mathrm{MHz}, \mathrm{CDCl}_{3}\right) ; \delta[\mathrm{ppm}]=170.5,143.7,139.9,137.8,124.2,74.2,72.5,53.9,52.0$, $39.8,39.3,39.1,37.3,36.8,36.4,35.6,35.1,33.5,32.2,30.5,27.8,27.7,22.8,21.8,21.7$, 21.5, 20.4, 19.1, 18.2, 15.6 .

IR: $\quad\left(\right.$ neat); $\tilde{v}\left[\mathrm{~cm}^{-1}\right]=2954(\mathrm{~m}), 2923(\mathrm{~s}), 2853(\mathrm{~m}), 1739(\mathrm{w}), 1458(\mathrm{w}), 1378(\mathrm{w})$.

HRMS: $\quad$ (ESI-TOF); $m / z$ calcd. for $\mathrm{C}_{30} \mathrm{H}_{48} \mathrm{O}_{3} \mathrm{Na}^{+}[\mathrm{M}+\mathrm{Na}]+:$ 479.3496, found: 479.3482 .

Opt. act. $[\mathrm{a}]_{\mathrm{D}}^{22}=+41.3\left(c=1.00, \mathrm{CHCl}_{3}\right)$. 

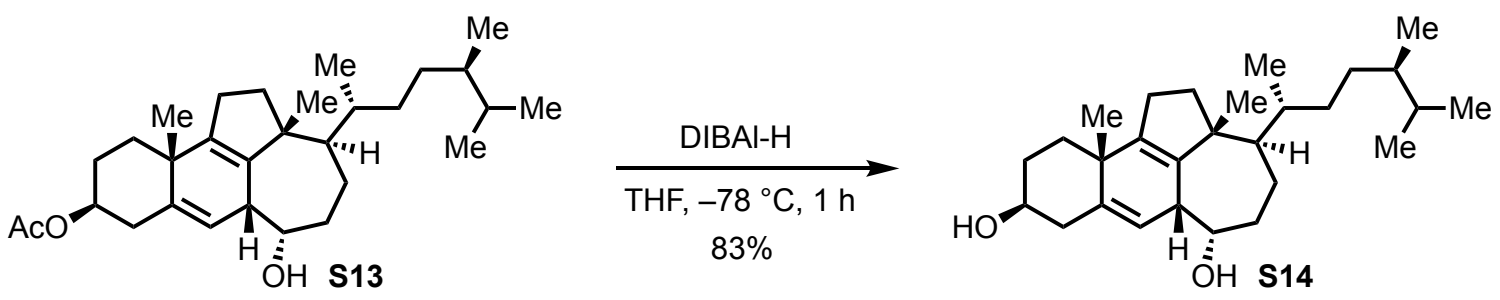

To a solution of acetate S13 (102 mg, $223 \mu \mathrm{mol}, 1.0$ eq.) in THF $(2.2 \mathrm{~mL})$ at $-78^{\circ} \mathrm{C}$ was added DIBAl-H (1 $\mathrm{m}$ in hexanes, $1.1 \mathrm{~mL}, 1.1 \mathrm{mmol}, 5.0$ eq.) and the resulting solution was stirred at this temperature for $1 \mathrm{~h}$. EtOAc ( $1 \mathrm{~mL})$ and Rochelle's salt $(1 / 2$ sat. aq., $5 \mathrm{~mL})$ were added carefully and the mixture was vigorously stirred for $30 \mathrm{~min}$ while warming to $25^{\circ} \mathrm{C}$. The aqueous phase was extracted with EtOAc $(3 \times 5 \mathrm{~mL})$ and the combined organic phases were washed with brine (sat., $15 \mathrm{~mL}$ ), dried over $\mathrm{MgSO}_{4}$, and the solvent was removed under reduced pressure. Column chromatography (silica gel, nhexane/EtOAc 1:1) gave diol S14 (76.7 mg, $185 \mu \mathrm{mol}, 83 \%$ ) as a colorless oil.

TLC: $\quad R_{\mathrm{f}}=0.29($ nhexane/EtOAc $1: 1)$.

1H-NMR: $\quad\left(500 \mathrm{MHz}, \mathrm{CDCl}_{3}\right) ; \delta[\mathrm{ppm}]=5.34(\mathrm{dd}, J=3.2,1.6 \mathrm{~Hz}, 1 \mathrm{H}), 3.83-3.75(\mathrm{~m}, 1 \mathrm{H}), 3.62-$ $3.51(\mathrm{~m}, 1 \mathrm{H}), 3.03-2.94(\mathrm{~m}, 1 \mathrm{H}), 2.44-2.37(\mathrm{~m}, 1 \mathrm{H}), 2.37-2.28(\mathrm{~m}, 2 \mathrm{H}), 2.28-2.20$ (m, 1H), $2.00-1.93(\mathrm{~m}, 1 \mathrm{H}), 1.93-1.86(\mathrm{~m}, 2 \mathrm{H}), 1.83(\mathrm{ddd}, J=12.0,7.5,2.3 \mathrm{~Hz}, 1 \mathrm{H})$, $1.73-1.65(\mathrm{~m}, 2 \mathrm{H}), 1.65-1.57(\mathrm{~m}, 3 \mathrm{H}), 1.57-1.46(\mathrm{~m}, 5 \mathrm{H}), 1.40-1.33(\mathrm{~m}, 2 \mathrm{H}), 1.27-$ $1.22(\mathrm{~m}, 1 \mathrm{H}), 1.22-1.15(\mathrm{~m}, 3 \mathrm{H}), 1.14(\mathrm{~s}, 3 \mathrm{H}), 0.96(\mathrm{~s}, 3 \mathrm{H}), 0.88(\mathrm{~d}, J=6.8 \mathrm{~Hz}, 3 \mathrm{H}), 0.84$ (d, $J=6.8 \mathrm{~Hz}, 3 \mathrm{H}), 0.79$ (dd, $J=6.8,0.8 \mathrm{~Hz}, 3 \mathrm{H}), 0.77(\mathrm{~d}, J=6.3 \mathrm{~Hz}, 3 \mathrm{H}$ ).

13C-NMR: $\quad\left(126 \mathrm{MHz}, \mathrm{CDCl}_{3}\right) ; \delta[\mathrm{ppm}]=144.4,141.0,137.4,123.2,72.5,72.4,53.9,52.3,41.4,39.7$, $39.3,39.1,36.8,36.7,35.7,35.1$, 33.5, 32.2, 31.6, 30.4, 27.9, 23.0, 21.8, 21.6, 20.4, 19.0, 18.2, 15.6 .

IR: $\quad$ (neat); $\tilde{v}\left[\mathrm{~cm}^{-1}\right]=3380(\mathrm{br} \mathrm{w}), 2954(\mathrm{~s}), 2927(\mathrm{~s}), 2869(\mathrm{~m}), 1706(\mathrm{~m}), 1459(\mathrm{~m}), 1376(\mathrm{~m})$, $1216(\mathrm{w}), 1058(\mathrm{~m}), 1007(\mathrm{~m}), 758(\mathrm{~m})$.

HRMS: $\quad$ (ESI-TOF); $\mathrm{m} / z$ calcd. for $\mathrm{C}_{28} \mathrm{H}_{46} \mathrm{O}_{2} \mathrm{Na}^{+}[\mathrm{M}+\mathrm{Na}]+:$ 437.3390, found: 437.3388.

Opt. act. $[\mathrm{a}]_{\mathrm{D}}^{22}=+45.3\left(c=1.00, \mathrm{CHCl}_{3}\right)$. 

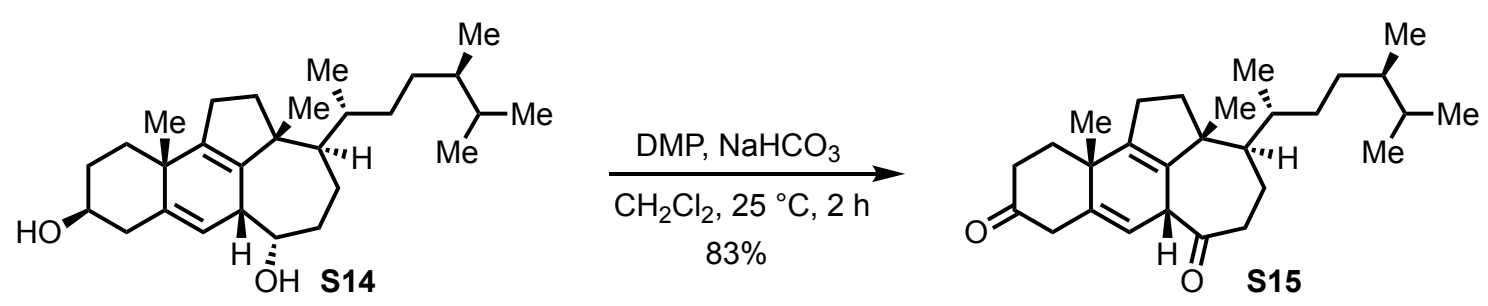

To a solution of diol $\mathrm{S} 14$ (64.4 mg, $155 \mu \mathrm{mol}, 1.0$ eq.) in $\mathrm{CH}_{2} \mathrm{Cl}_{2}(1.5 \mathrm{~mL})$ were added $\mathrm{NaHCO}_{3}(84.6 \mathrm{mg}$, $1.01 \mathrm{mmol}, 6.5$ eq.) and Dess-Martin periodinane (197 mg, $465 \mu \mathrm{mol}, 3.0$ eq.), and the resulting suspension was stirred at $25^{\circ} \mathrm{C}$. After $45 \mathrm{~min}, \mathrm{Na}_{2} \mathrm{~S}_{2} \mathrm{O}_{3}$ (sat. aq., $3 \mathrm{~mL}$ ) was added and after further stirring for $15 \mathrm{~min}$, the aqueous phase was extracted with $\mathrm{CH}_{2} \mathrm{Cl}_{2}(3 \times 10 \mathrm{~mL})$. The combined organic phases were washed sequentially with $\mathrm{NaHCO}_{3}$ (sat. aq., $15 \mathrm{~mL}$ ) and brine (sat., $15 \mathrm{~mL}$ ), dried over $\mathrm{MgSO}_{4}$, and the solvent was removed under reduced pressure. Column chromatography (silica gel, nhexane/EtOAc 5:1) gave dione $\mathbf{S} 15$ (53.0 mg, $129 \mu \mathrm{mol}, 83 \%)$ as a colorless oil.

TLC: $\quad R_{\mathrm{f}}=0.25$ (nhexane/EtOAc 5:1).

1H-NMR: $\quad\left(500 \mathrm{MHz}, \mathrm{CDCl}_{3}\right) ; \delta[\mathrm{ppm}]=5.22(\mathrm{t}, J=2.7 \mathrm{~Hz}, 1 \mathrm{H}), 3.54(\mathrm{t}, J=3.2 \mathrm{~Hz}, 1 \mathrm{H}), 3.36(\mathrm{dt}, J=$ 16.1, 3.2 Hz, 1H), 2.97 (dd, $J=16.1,2.2 \mathrm{~Hz}, 1 \mathrm{H}$ ), 2.59 (ddd, $J=15.4,13.6,6.5 \mathrm{~Hz}, 1 \mathrm{H}$ ), $2.45-2.32(\mathrm{~m}, 4 \mathrm{H}), 2.23-2.11(\mathrm{~m}, 2 \mathrm{H}), 2.03(\mathrm{ddd}, J=13.2,6.5,2.4 \mathrm{~Hz}, 1 \mathrm{H}), 1.80-1.72$ $(\mathrm{m}, 1 \mathrm{H}), 1.66-1.60(\mathrm{~m}, 1 \mathrm{H}), 1.60-1.55(\mathrm{~m}, 1 \mathrm{H}), 1.54-1.50(\mathrm{~m}, 1 \mathrm{H}), 1.50-1.45(\mathrm{~m}$, $1 \mathrm{H}), 1.40(\mathrm{~s}, 3 \mathrm{H}), 1.35-1.29(\mathrm{~m}, 1 \mathrm{H}), 1.29-1.23(\mathrm{~m}, 2 \mathrm{H}), 1.22-1.13(\mathrm{~m}, 3 \mathrm{H}), 0.95-$ $0.91(\mathrm{~m}, 6 \mathrm{H}), 0.89-0.85(\mathrm{~m}, 1 \mathrm{H}), 0.84(\mathrm{~d}, J=6.8 \mathrm{~Hz}, 3 \mathrm{H}), 0.78(\mathrm{~d}, J=6.8 \mathrm{~Hz}, 3 \mathrm{H}), 0.76$ (d, $J=6.3 \mathrm{~Hz}, 3 \mathrm{H})$.

13C-NMR: $\quad\left(126 \mathrm{MHz}, \mathrm{CDCl}_{3}\right) ; \delta[\mathrm{ppm}]=211.8,207.9,142.3,139.7,135.8,119.2,53.7,51.5,50.4$, 48.2, 41.2, 39.1, 38.1, 38.0, 37.1, 35.9, 35.4, 33.4, 32.2, 30.8, 27.6, 22.6, 22.1, 21.4, 20.4, 19.6, 18.2, 15.6.

IR: $\quad\left(\right.$ neat); $\tilde{v}\left[\mathrm{~cm}^{-1}\right]=2953(\mathrm{~m}), 2923(\mathrm{~s}), 2853(\mathrm{~m}), 1717(\mathrm{~m}), 1459(\mathrm{w}), 1377(\mathrm{w}), 1239(\mathrm{w})$.

HRMS: (ESI-TOF); $\mathrm{m} / \mathrm{z}$ calcd. for $\mathrm{C}_{28} \mathrm{H}_{42} \mathrm{O}_{2} \mathrm{Na}^{+}[\mathrm{M}+\mathrm{Na}]+:$ 433.3077, found: 433.3074 .

Opt. act. $[a]_{D}^{21}=-27.1\left(c=1.00, \mathrm{CHCl}_{3}\right)$. 

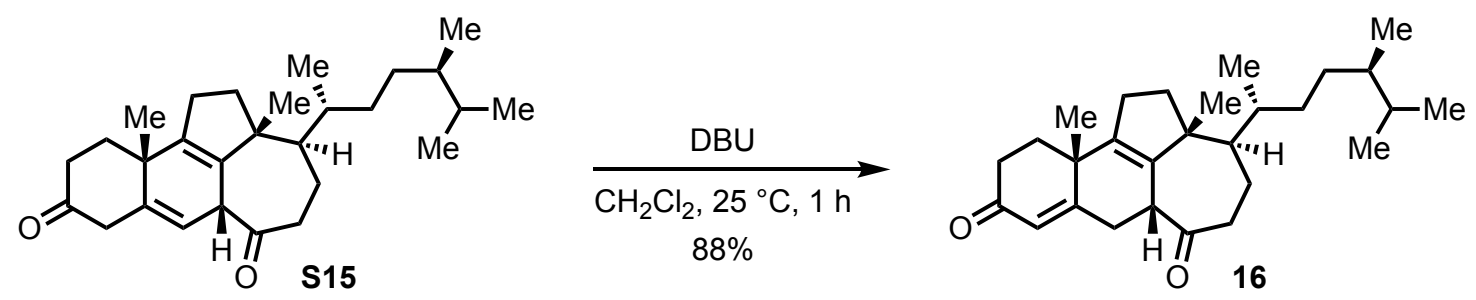

To a solution of dione $\mathbf{S} 15$ (36.9 mg, $89.9 \mu \mathrm{mol}, 1.0$ eq.) in $\mathrm{CH}_{2} \mathrm{Cl}_{2}$ (1.5 mL) was added 1,8diazabicyclo[5.4.0]undec-7-ene $\left(2.7 \mathrm{mg}, 18 \mu \mathrm{mol}, 0.2 \mathrm{eq}\right.$.) and the resulting solution was stirred at $25{ }^{\circ} \mathrm{C}$ for $1 \mathrm{~h}$. The reaction mixture was diluted with $\mathrm{CH}_{2} \mathrm{Cl}_{2}(2 \mathrm{~mL})$ and $\mathrm{NH}_{4} \mathrm{Cl}$ (sat. aq., $5 \mathrm{~mL}$ ) was added. The aqueous phase was extracted with $\mathrm{CH}_{2} \mathrm{Cl}_{2}(3 \times 10 \mathrm{~mL})$ and the combined organic phases were washed with brine (sat., $25 \mathrm{~mL}$ ), dried over $\mathrm{MgSO}_{4}$, and the solvent was removed under reduced pressure. Column chromatography (silica gel, nhexane/EtOAc 4:1 $\rightarrow$ 3:1) gave enone 16 (32.4 mg, $78.9 \mu \mathrm{mol}, 88 \%$ ) as a lightyellow oil.

M.p. $\quad 148-150{ }^{\circ} \mathrm{C}\left(\mathrm{CHCl}_{3}\right)$

TLC: $\quad R_{\mathrm{f}}=0.26($ nhexane/EtOAc $3: 1)$.

1H-NMR: $\quad\left(500 \mathrm{MHz}, \mathrm{CDCl}_{3}\right) ; \delta[\mathrm{ppm}]=5.88-5.84(\mathrm{~m}, 1 \mathrm{H}), 3.26-3.19(\mathrm{~m}, 1 \mathrm{H}), 2.86(\mathrm{dd}, J=13.1$, $1.6 \mathrm{~Hz}, 1 \mathrm{H}), 2.57-2.47(\mathrm{~m}, 3 \mathrm{H}), 2.47-2.38(\mathrm{~m}, 2 \mathrm{H}), 2.32-2.27(\mathrm{~m}, 2 \mathrm{H}), 2.21-2.12(\mathrm{~m}$, $1 \mathrm{H}), 2.00-1.93(\mathrm{~m}, 2 \mathrm{H}), 1.76-1.69(\mathrm{~m}, 2 \mathrm{H}), 1.65-1.61(\mathrm{~m}, 2 \mathrm{H}), 1.58-1.50(\mathrm{~m}, 2 \mathrm{H})$, $1.40-1.31(\mathrm{~m}, 1 \mathrm{H}), 1.29(\mathrm{~s}, 3 \mathrm{H}), 1.26-1.22(\mathrm{~m}, 2 \mathrm{H}), 1.21-1.18(\mathrm{~m}, 2 \mathrm{H}), 1.08(\mathrm{~s}, 3 \mathrm{H})$, $0.93(\mathrm{~d}, J=6.9 \mathrm{~Hz}, 3 \mathrm{H}), 0.85(\mathrm{~d}, J=6.8 \mathrm{~Hz}, 3 \mathrm{H}), 0.80(\mathrm{~d}, J=6.9 \mathrm{~Hz}, 3 \mathrm{H}), 0.77(\mathrm{~d}, J=6.4$ $\mathrm{Hz}, 3 \mathrm{H})$.

13C-NMR: $\quad\left(126 \mathrm{MHz}, \mathrm{CDCl}_{3}\right) ; \delta[\mathrm{ppm}]=211.3,198.5,166.8,143.3,137.7,126.0,55.1,53.6,46.0$, $44.9,39.1,38.0,37.5,35.0,34.1,33.6,33.4,32.8,32.1,30.4,27.1,22.3,21.4,20.3(2 \mathrm{C})$, 19.9, 18.1, 15.6 .

IR: $\quad\left(\right.$ neat); $\tilde{v}\left[\mathrm{~cm}^{-1}\right]=2953(\mathrm{~s}), 2926(\mathrm{~s}), 2867(\mathrm{~m}), 1707(\mathrm{~s}), 1669(\mathrm{~s}), 1456(\mathrm{~m}), 1376(\mathrm{~m})$, $1235(\mathrm{~m}), 1183(\mathrm{w}), 999(\mathrm{w}), 948(\mathrm{w}), 869(\mathrm{w})$.

HRMS: (ESI-TOF); $\mathrm{m} / \mathrm{z}$ calcd. $\mathrm{C}_{28} \mathrm{H}_{42} \mathrm{O}_{2} \mathrm{Na}^{+}[\mathrm{M}+\mathrm{Na}]^{+}: 433.3077$, found: 433.3074.

Opt. act. $[a]_{D}^{23}=+173.8\left(c=1.00, \mathrm{CHCl}_{3}\right)$. 
3ß-Hydroxy-13(14 $\rightarrow 8), 14(8 \rightarrow 7)$ diabeo-7ß(H)-campesta-4,8-dien-14-one(S16)
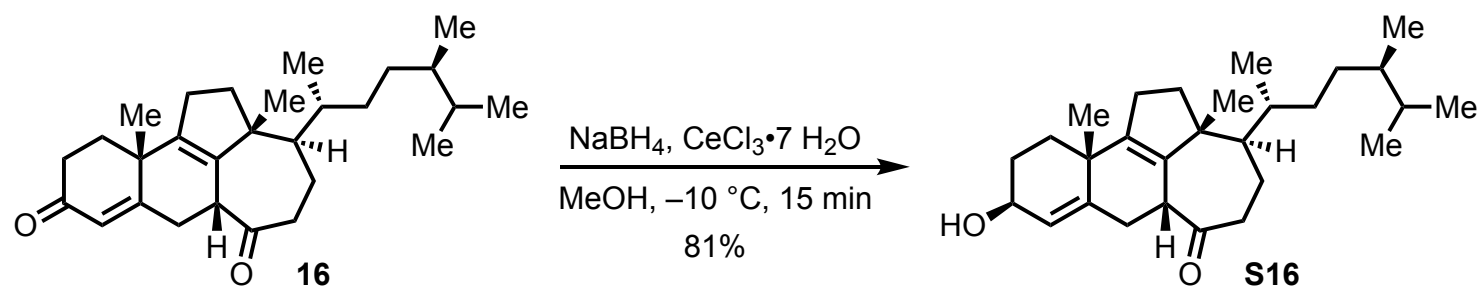

To a solution of enone 16 (32.4 mg, $78.9 \mu \mathrm{mol}, 1.0$ eq.) in $\mathrm{MeOH}(2 \mathrm{~mL})$ at $-10{ }^{\circ} \mathrm{C}$ were added $\mathrm{CeCl}_{3} \cdot 7 \mathrm{H}_{2} \mathrm{O}$ (73.5 mg, $197 \mu \mathrm{mol}, 2.5$ eq.) and $\mathrm{NaBH}_{4}(1.8 \mathrm{mg}, 47 \mu \mathrm{mol}, 0.6$ eq.) in three portions over $15 \mathrm{~min}$. The reaction mixture was diluted with $\mathrm{EtOAc}(2 \mathrm{~mL})$ and $\mathrm{HCl}\left(1 \mathrm{M}\right.$ in $\left.\mathrm{H}_{2} \mathrm{O}, 5 \mathrm{~mL}\right)$ was added. The aqueous phase was extracted with EtOAc $(3 \times 5 \mathrm{~mL})$ and the combined organic phases were washed sequentially with $\mathrm{NaHCO}_{3}$ (sat. aq., $15 \mathrm{~mL}$ ) and brine (sat., $15 \mathrm{~mL}$ ), and dried over $\mathrm{MgSO}_{4}$. The solvent was removed under reduced pressure. Column chromatography (silica gel, nhexane/EtOAc 2:1) gave allylic alcohol S16 (26.4 mg, $63.9 \mu \mathrm{mol}, 81 \%$ ) as a colorless oil.

TLC: $\quad R_{\mathrm{f}}=0.28($ nhexane/EtOAc 2:1).

1H-NMR: $\quad\left(500 \mathrm{MHz}, \mathrm{CDCl}_{3}\right) ; \delta[\mathrm{ppm}]=5.36(\mathrm{q}, J=1.7 \mathrm{~Hz}, 1 \mathrm{H}), 4.20-4.12(\mathrm{~m}, 1 \mathrm{H}), 3.00-2.94(\mathrm{~m}$, $1 \mathrm{H}), 2.61(\mathrm{dd}, J=12.9,1.6 \mathrm{~Hz}, 1 \mathrm{H}), 2.42-2.33(\mathrm{~m}, 2 \mathrm{H}), 2.26-2.15(\mathrm{~m}, 3 \mathrm{H}), 2.03-1.93$ $(\mathrm{m}, 2 \mathrm{H}), 1.68(\mathrm{dt}, J=13.1,3.6 \mathrm{~Hz}, 1 \mathrm{H}), 1.64-1.53(\mathrm{~m}, 6 \mathrm{H}), 1.53-1.48(\mathrm{~m}, 2 \mathrm{H}), 1.38-$ $1.31(\mathrm{~m}, 2 \mathrm{H}), 1.26-1.21(\mathrm{~m}, 2 \mathrm{H}), 1.19(\mathrm{~s}, 3 \mathrm{H}), 1.00(\mathrm{~s}, 3 \mathrm{H}), 0.92(\mathrm{~d}, J=6.9 \mathrm{~Hz}, 3 \mathrm{H}), 0.89$ $-0.86(\mathrm{~m}, 1 \mathrm{H}), 0.84(\mathrm{~d}, J=6.8 \mathrm{~Hz}, 3 \mathrm{H}), 0.79(\mathrm{~d}, J=6.8 \mathrm{~Hz}, 3 \mathrm{H}), 0.76(\mathrm{~d}, J=6.3 \mathrm{~Hz}, 3 \mathrm{H})$.

13C-NMR: $\quad\left(126 \mathrm{MHz}, \mathrm{CDCl}_{3}\right) ; \delta[\mathrm{ppm}]=214.2,145.2,142.8,137.4,125.7,67.8,54.1,53.5,46.6$, $45.5,39.1,37.9,36.4,35.1,33.4,33.1,32.8,32.2,30.5,28.8,27.1,23.9,21.4,20.4(2 \mathrm{C})$, 20.3, 18.2, 15.6 .

IR: $\quad\left(\right.$ neat); $\tilde{v}\left[\mathrm{~cm}^{-1}\right]=3383(\mathrm{br} \mathrm{w}), 2954(\mathrm{~s}), 2929(\mathrm{~s}), 2868(\mathrm{~m}), 1707(\mathrm{~m}), 1458(\mathrm{~m}), 1375(\mathrm{w})$, $1066(w), 1012(w), 860(w)$.

HRMS: (ESI-TOF); $\mathrm{m} / z$ calcd. for $\mathrm{C}_{28} \mathrm{H}_{44} \mathrm{O}_{2} \mathrm{Na}^{+}[\mathrm{M}+\mathrm{Na}]^{+}: 435.3234$, found: 435.3229 .

Opt. act. $[a]_{D}^{21}=+41.4\left(c=1.00, \mathrm{CHCl}_{3}\right)$. 

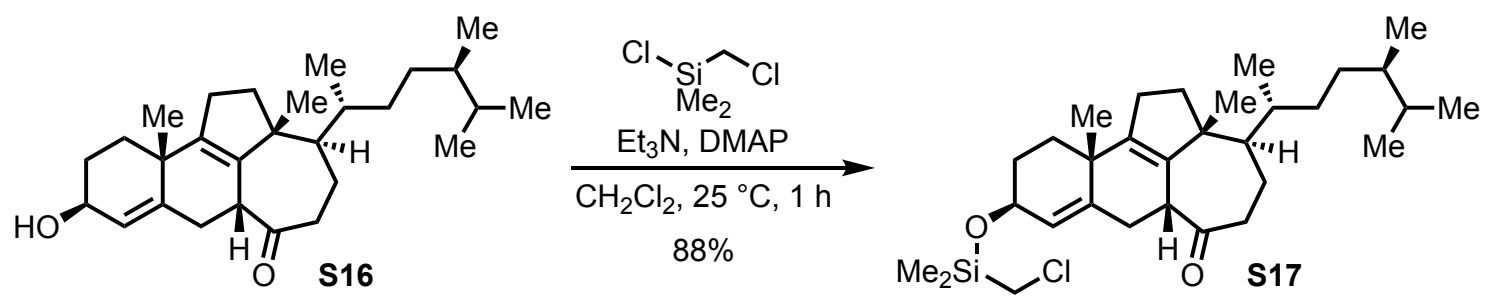

To a solution of allylic alcohol $\mathbf{S 1 6}\left(17.6 \mathrm{mg}, 42 \mu \mathrm{mol}, 1.0\right.$ eq.) in $\mathrm{CH}_{2} \mathrm{Cl}_{2}(1.5 \mathrm{~mL})$ at $0{ }^{\circ} \mathrm{C}$ were added $\mathrm{Et}_{3} \mathrm{~N}$ (116 $\mu \mathrm{L}, 840 \mu \mathrm{mol}, 20$ eq.), 4-(dimethylamino)pyridine (1.0 mg, $8.4 \mu \mathrm{mol}, 0.2$ eq.) and (chloromethyl)chlorodimethylsilane ( $83 \mu \mathrm{L}, 0.63 \mathrm{mmol}, 15$ eq.), and the resulting solution was stirred at $25^{\circ} \mathrm{C}$ for $1 \mathrm{~h}$. The mixture was diluted with $\mathrm{CH}_{2} \mathrm{Cl}_{2}\left(2.5 \mathrm{~mL}\right.$ ) and $\mathrm{NH}_{4} \mathrm{Cl}$ (sat. aq., $5 \mathrm{~mL}$ ) was added. The aqueous phase was extracted with $\mathrm{CH}_{2} \mathrm{Cl}_{2}(3 \times 5 \mathrm{~mL})$ and the combined organic phases were washed sequentially with $\mathrm{NH}_{4} \mathrm{Cl}$ (sat. aq., $4 \times 20 \mathrm{~mL}$ ), $\mathrm{NaHCO}_{3}$ (sat. aq., $15 \mathrm{~mL}$ ), and brine (sat., $15 \mathrm{~mL}$ ). The organic phase was dried over $\mathrm{MgSO}_{4}$, the solvent was removed under reduced pressure, and column chromatography (silica gel, nhexane/EtOAc 19:1) gave chloride $\mathbf{S} 17$ (19.3 mg, $37.2 \mu \mathrm{mol}, 88 \%$ ) as a colorless oil.

TLC: $\quad R_{\mathrm{f}}=0.19($ nhexane/EtOAc 19:1).

1H-NMR: $\quad\left(500 \mathrm{MHz}, \mathrm{CDCl}_{3}\right) ; \delta[\mathrm{ppm}]=5.31-5.25(\mathrm{~m}, 1 \mathrm{H}), 4.24(\mathrm{ddt}, J=8.6,6.5,1.9 \mathrm{~Hz}, 1 \mathrm{H}), 3.00$ - $2.94(\mathrm{~m}, 1 \mathrm{H}), 2.78(\mathrm{~s}, 2 \mathrm{H}), 2.60(\mathrm{dd}, J=12.9,1.5 \mathrm{~Hz}, 1 \mathrm{H}), 2.44-2.32(\mathrm{~m}, 2 \mathrm{H}), 2.26-$ $2.15(\mathrm{~m}, 3 \mathrm{H}), 1.96(\mathrm{ddd}, J=12.6,7.4,3.6 \mathrm{~Hz}, 1 \mathrm{H}), 1.92-1.86(\mathrm{~m}, 1 \mathrm{H}), 1.72-1.57(\mathrm{~m}$, $6 \mathrm{H}), 1.57-1.48(\mathrm{~m}, 3 \mathrm{H}), 1.38-1.30(\mathrm{~m}, 2 \mathrm{H}), 1.26-1.20(\mathrm{~m}, 2 \mathrm{H}), 1.18(\mathrm{~s}, 3 \mathrm{H}), 1.00(\mathrm{~s}$, $3 \mathrm{H}), 0.92(\mathrm{~d}, J=6.9 \mathrm{~Hz}, 3 \mathrm{H}), 0.90-0.86(\mathrm{~m}, 1 \mathrm{H}), 0.84(\mathrm{~d}, J=6.8 \mathrm{~Hz}, 3 \mathrm{H}), 0.79(\mathrm{~d}, J=6.8$ $\mathrm{Hz}, 3 \mathrm{H}), 0.76(\mathrm{~d}, J=6.3 \mathrm{~Hz}, 3 \mathrm{H}), 0.24(\mathrm{~s}, 6 \mathrm{H})$.

13C-NMR: $\quad\left(126 \mathrm{MHz}, \mathrm{CDCl}_{3}\right) ; \delta[\mathrm{ppm}]=214.1,145.2,142.5,137.3,125.5,69.1,54.2,53.4,46.6$, $45.4,39.0,37.9,36.3,35.1$, 33.4, 33.1, 32.7, 32.1, 30.4, 30.1, 28.9, 27.0, 23.7, 21.3, 20.3, $20.3(2 \mathrm{C}), 18.1,15.6,-2.7,-2.8$.

IR: $\quad\left(\right.$ neat); $\tilde{v}\left[\mathrm{~cm}^{-1}\right]=2953(\mathrm{~m}), 2923(\mathrm{~s}), 2853(\mathrm{~m}), 1739(\mathrm{~m}), 1711(\mathrm{~m}), 1458(\mathrm{~m}), 1376(\mathrm{~m})$, $1253(w), 1217(w), 1074(m), 986(w), 888(w), 822(w)$.

HRMS: (ESI-TOF); $\mathrm{m} / \mathrm{z}$ calcd. for $\mathrm{C}_{31} \mathrm{H}_{51} \mathrm{ClO}_{2} \mathrm{SiNa}^{+}[\mathrm{M}+\mathrm{Na}]^{+}: 541.3239$, found: 541.3225

Opt. act. $\quad[\mathrm{a}]_{\mathrm{D}}^{23}=+25.7\left(c=1.00, \mathrm{CHCl}_{3}\right)$. 


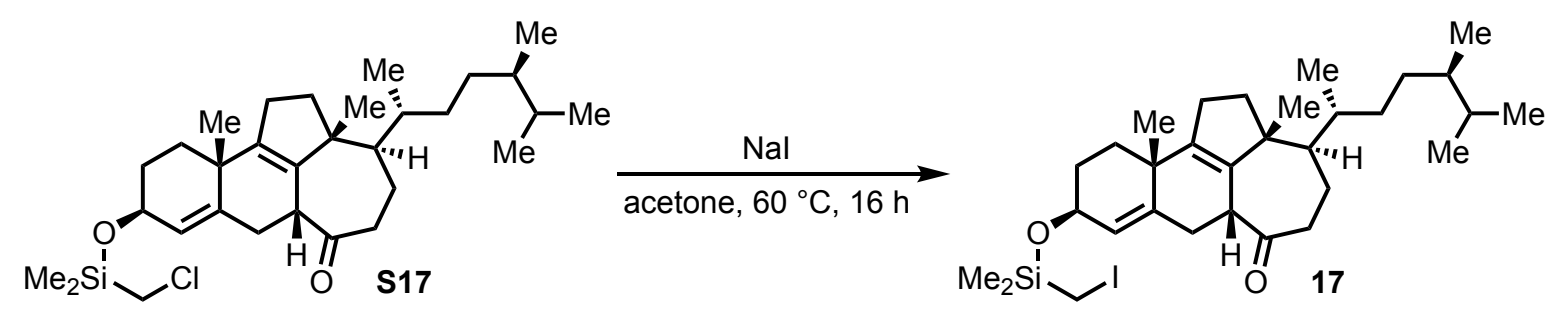

Sodium iodide (previously dried under reduced pressure at $120^{\circ} \mathrm{C}$ for $24 \mathrm{~h}, 279 \mathrm{mg}, 1.86 \mathrm{mmol}, 50$ eq.) was added to a solution of chloride $\mathbf{S} 17$ (19.3 mg, $37.2 \mu \mathrm{mol}, 1.0$ eq.) in acetone ( $2 \mathrm{~mL}$ ) and the mixture was stirred at $60^{\circ} \mathrm{C}$ for $16 \mathrm{~h}$. The reaction mixture was allowed to cool to $25^{\circ} \mathrm{C}$, diluted with $\mathrm{H}_{2} \mathrm{O}(5 \mathrm{~mL})$ and EtOAc $(5 \mathrm{~mL})$, and the aqueous phase was extracted with EtOAc $(3 \times 10 \mathrm{~mL})$. The combined organic phases were washed with brine (sat., $30 \mathrm{~mL}$ ), dried over $\mathrm{MgSO}_{4}$ and the solvent was removed under reduced pressure. The crude product 17 was used in the next step without further purification.

TLC: $\quad R_{\mathrm{f}}=0.20($ nhexane/EtOAc 19:1).

1H-NMR: $\quad\left(600 \mathrm{MHz}, \mathrm{CDCl}_{3}\right) ; \delta[\mathrm{ppm}]=5.29(\mathrm{dt}, J=2.6,1.4 \mathrm{~Hz}, 1 \mathrm{H}), 4.23(\mathrm{ddt}, J=9.1,6.7,2.0 \mathrm{~Hz}$, $1 \mathrm{H}), 3.00-2.94(\mathrm{~m}, 1 \mathrm{H}), 2.60(\mathrm{dd}, J=12.8,1.6 \mathrm{~Hz}, 1 \mathrm{H}), 2.43-2.32(\mathrm{~m}, 2 \mathrm{H}), 2.24-2.17$ (m, 3H), 2.03 (s, 2H), 1.96 (ddd, $J=12.7,7.5,3.4 \mathrm{~Hz}, 1 \mathrm{H}), 1.93-1.86(\mathrm{~m}, 1 \mathrm{H}), 1.68-$ $1.57(\mathrm{~m}, 6 \mathrm{H}), 1.54-1.46(\mathrm{~m}, 2 \mathrm{H}), 1.36-1.30(\mathrm{~m}, 3 \mathrm{H}), 1.26-1.21(\mathrm{~m}, 2 \mathrm{H}), 1.18(\mathrm{~s}, 3 \mathrm{H})$, $1.00(\mathrm{~s}, 3 \mathrm{H}), 0.92(\mathrm{~d}, J=6.9 \mathrm{~Hz}, 3 \mathrm{H}), 0.90-0.86(\mathrm{~m}, 1 \mathrm{H}), 0.84(\mathrm{~d}, J=6.8 \mathrm{~Hz}, 3 \mathrm{H}), 0.79$ $(\mathrm{d}, J=6.8 \mathrm{~Hz}, 3 \mathrm{H}), 0.76(\mathrm{~d}, J=6.4 \mathrm{~Hz}, 3 \mathrm{H}), 0.30(\mathrm{~d}, J=1.1 \mathrm{~Hz}, 6 \mathrm{H})$.

13C-NMR: $\quad\left(151 \mathrm{MHz}, \mathrm{CDCl}_{3}\right) ; \delta[\mathrm{ppm}]=214.2,145.2,142.5,137.3,125.6,69.2,54.2,53.5,46.6$, 45.5, 39.1, 37.9, 36.3, 35.1, 33.4, 33.1, 32.8, 32.2, 30.5, 29.0, 27.1, 23.8, 21.4, 20.4, 20.4 (2C), 18.2, 15.6, -1.8, -1.9, -13.9.

IR: $\quad\left(\right.$ neat); $\tilde{v}\left[\mathrm{~cm}^{-1}\right]=2953(\mathrm{~s}), 2924(\mathrm{~s}), 2853(\mathrm{~m}), 1708(\mathrm{~m}), 1458(\mathrm{~m}), 1376(\mathrm{w}), 1254(\mathrm{~m})$, $1070(\mathrm{~m}), 884(\mathrm{w}), 834(\mathrm{~m}), 803(\mathrm{~m})$.

HRMS: (ESI-TOF); $m / z$ calcd. for $\mathrm{C}_{31} \mathrm{H}_{51} \mathrm{lO}_{2} \mathrm{SiNa}^{+}[\mathrm{M}+\mathrm{Na}]^{+}:$633.2595, found: 633.2565 .

Opt. act. $[\mathrm{a}]_{\mathrm{D}}^{24}=+18.0\left(c=1.00, \mathrm{CHCl}_{3}\right)$. 

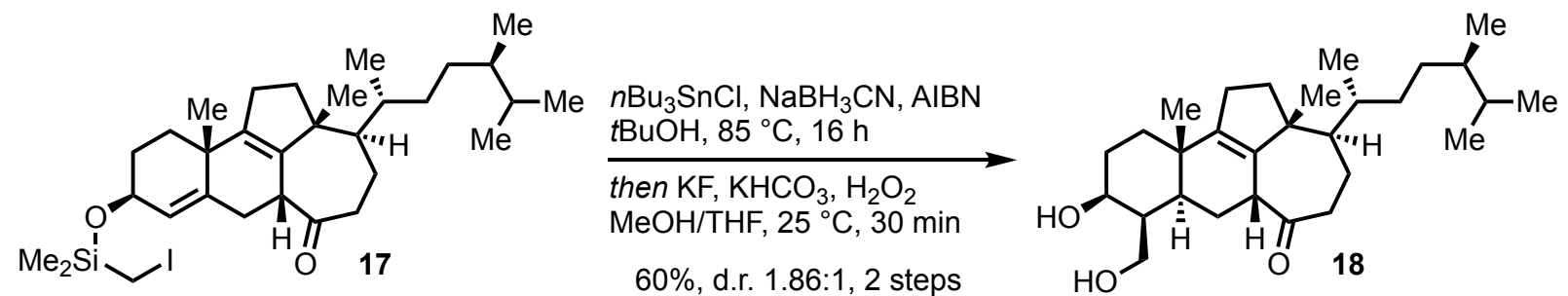

A solution of crude iodide $17(37.2 \mu \mathrm{mol}, 1.0$ eq.), azobisisobutyronitrile $(0.6 \mathrm{mg}, 3.7 \mu \mathrm{mol}, 0.1 \mathrm{eq}$.$) ,$ $n \mathrm{Bu}_{3} \mathrm{SnCl}$ (2.0 $\mu \mathrm{L}, 7.4 \mu \mathrm{mol}, 0.2$ eq.) and $\mathrm{NaBH}_{3} \mathrm{CN}$ (4.7 mg, $74 \mu \mathrm{mol}, 2.0$ eq.) in $t \mathrm{BuOH}$ was degassed applying three freeze-pump-thaw cycles and stirred at $85^{\circ} \mathrm{C}$ for $16 \mathrm{~h}$. The reaction mixture was cooled to $25^{\circ} \mathrm{C}$ and diluted with EtOAc $(5 \mathrm{~mL})$ and brine (sat., $\left.15 \mathrm{~mL}\right)$. The aqueous phase was extracted with EtOAc $(3 \times 10 \mathrm{~mL})$ and the combined organic phases were washed with brine (sat., $30 \mathrm{~mL})$, dried over $\mathrm{MgSO}_{4}$, and the solvent was removed under reduced pressure. The residue was taken up in $\mathrm{MeOH} / \mathrm{THF}(2: 1,1 \mathrm{~mL})$ and $\mathrm{KF}$ (21.6 mg, $372 \mu \mathrm{mol}, 10$ eq.), $\mathrm{KHCO}_{3}$ (37.2 mg, $372 \mu \mathrm{mol}, 10$ eq.), and $\mathrm{H}_{2} \mathrm{O}_{2}$ (35\% w/w, $0.75 \mathrm{~mL}$ ) were added at $25^{\circ} \mathrm{C}$ and the reaction mixture was stirred at this temperature for $30 \mathrm{~min}$. It was diluted with EtOAc ( $5 \mathrm{~mL}$ ) and cooled in a $\mathrm{H}_{2} \mathrm{O}$ bath to maintain the temperature at $25^{\circ} \mathrm{C}$ before $\mathrm{Na}_{2} \mathrm{~S}_{2} \mathrm{O}_{3}$ (sat. aq., $5 \mathrm{~mL})$ was added carefully. The aqueous phase was extracted with EtOAc $(3 \times 10 \mathrm{~mL})$, the combined organic phases were washed sequentially with $\mathrm{NaHCO}_{3}$ (sat. aq., $30 \mathrm{~mL}$ ) and brine (sat., $30 \mathrm{~mL}$ ), dried over $\mathrm{MgSO}_{4}$, and the solvent was removed under reduced pressure. Column chromatography (silica gel, nhexane/EtOAc 2:1 $\rightarrow$ 1:1 $\rightarrow$ 1:2) gave 5a-epimer 18 (6.4 mg, $14 \mu \mathrm{mol}, 39 \%)$ and 5ß-epimer S18 (3.5 mg, $7.8 \mu \mathrm{mol}, 21 \%)$ as colorless oils.

\section{major 5a-epimer 18}

TLC: $\quad R_{\mathrm{f}}=0.29($ nhexane/EtOAc 1:2).

1H-NMR: $\quad\left(600 \mathrm{MHz}, \mathrm{CDCl}_{3}\right) ; \delta[\mathrm{ppm}]=4.02(\mathrm{t}, J=10.4 \mathrm{~Hz}, 1 \mathrm{H}), 3.94(\mathrm{dt}, J=9.1,6.2 \mathrm{~Hz}, 1 \mathrm{H}), 3.55$ (d, $J=11.0 \mathrm{~Hz}, 1 \mathrm{H}$ ), $3.14-3.08(\mathrm{~m}, 1 \mathrm{H}), 2.54$ (ddd, $J=16.5,6.1,2.3 \mathrm{~Hz}, 1 \mathrm{H}), 2.37-2.28$ (m, 2H), 2.17 (td, $J=7.3,2.3 \mathrm{~Hz}, 2 \mathrm{H}), 1.99$ (d, $J=11.5 \mathrm{~Hz}, 1 \mathrm{H}), 1.77-1.71(\mathrm{~m}, 4 \mathrm{H}), 1.66$ $-1.62(\mathrm{~m}, 4 \mathrm{H}), 1.61-1.58(\mathrm{~m}, 1 \mathrm{H}), 1.56-1.50(\mathrm{~m}, 2 \mathrm{H}), 1.41-1.34(\mathrm{~m}, 1 \mathrm{H}), 1.23-1.17$ $(\mathrm{m}, 4 \mathrm{H}), 1.17-1.13(\mathrm{~m}, 1 \mathrm{H}), 0.98(\mathrm{~s}, 3 \mathrm{H}), 0.91(\mathrm{~d}, J=6.8 \mathrm{~Hz}, 3 \mathrm{H}), 0.90-0.87(\mathrm{~m}, 1 \mathrm{H})$, $0.85(\mathrm{~d}, J=6.9 \mathrm{~Hz}, 3 \mathrm{H}), 0.79(\mathrm{~d}, J=6.6 \mathrm{~Hz}, 3 \mathrm{H}), 0.79(\mathrm{~s}, 3 \mathrm{H}), 0.78(\mathrm{~d}, J=6.8 \mathrm{~Hz}, 3 \mathrm{H})$.

${ }^{13} \mathrm{C}-N M R: \quad\left(151 \mathrm{MHz}, \mathrm{CDCl}_{3}\right) ; \delta[\mathrm{ppm}]=214.1,147.1,137.2,74.5,60.3,55.8,53.8,48.2,45.9,45.5$, 41.6, 39.1, 38.6, 35.2, 35.1, 34.7, 33.4, 32.2, 30.5, 27.6, 26.9, 26.9, 21.6, 20.4, 20.0, 19.9, 19.1, 18.2, 15.6 .

IR: $\quad\left(\right.$ neat); $\tilde{v}\left[\mathrm{~cm}^{-1}\right]=2952(\mathrm{~m}), 2921(\mathrm{~s}), 2852(\mathrm{~m}), 1740(\mathrm{w}), 1459(\mathrm{w}), 1377(\mathrm{w}), 1243(\mathrm{w})$, $1082(w), 761(w)$. 
HRMS: $\quad$ (ESI-TOF); $\mathrm{m} / z$ calcd. $\mathrm{C}_{29} \mathrm{H}_{48} \mathrm{O}_{3} \mathrm{Na}^{+}[\mathrm{M}+\mathrm{Na}]^{+}: 467.3496$, found:467.3486.

Opt. act. $[\mathrm{a}]_{\mathrm{D}}^{22}=+40.6\left(c=0.54, \mathrm{CHCl}_{3}\right)$.

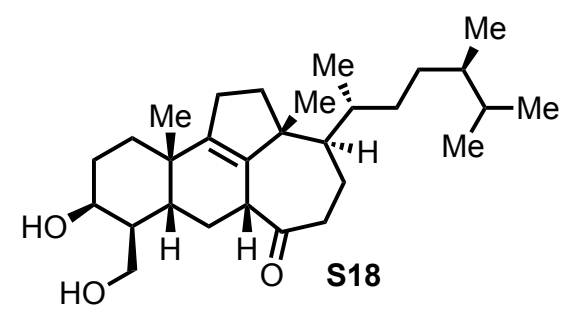

minor $5 \beta$-epimer $\mathbf{S 1 8}$

TLC: $\quad R_{\mathrm{f}}=0.26($ nhexane/EtOAc $1: 1)$.

1H-NMR: $\quad\left(700 \mathrm{MHz}, \mathrm{CDCl}_{3}\right) ; \delta[\mathrm{ppm}]=4.16-4.10(\mathrm{~m}, 1 \mathrm{H}), 3.85(\mathrm{dd}, J=11.1,5.2 \mathrm{~Hz}, 1 \mathrm{H}), 3.73(\mathrm{dd}$, $J=11.1,2.3 \mathrm{~Hz}, 1 \mathrm{H}), 2.80(\mathrm{~d}, J=8.0 \mathrm{~Hz}, 1 \mathrm{H}), 2.52-2.41(\mathrm{~m}, 3 \mathrm{H}), 2.34-2.27(\mathrm{~m}, 1 \mathrm{H})$, $2.27-2.20(\mathrm{~m}, 1 \mathrm{H}), 2.02$ (ddd, $J=14.5,8.0,3.8 \mathrm{~Hz}, 1 \mathrm{H}), 1.96$ (ddd, $J=13.0,8.9,4.2 \mathrm{~Hz}$, $1 \mathrm{H}), 1.89(\mathrm{dt}, J=11.7,3.6 \mathrm{~Hz}, 1 \mathrm{H}), 1.73(\mathrm{td}, J=13.9,3.2 \mathrm{~Hz}, 1 \mathrm{H}), 1.70-1.61(\mathrm{~m}, 3 \mathrm{H})$, $1.61-1.57(\mathrm{~m}, 1 \mathrm{H}), 1.52-1.45(\mathrm{~m}, 2 \mathrm{H}), 1.37-1.32(\mathrm{~m}, 2 \mathrm{H}), 1.30-1.24(\mathrm{~m}, 3 \mathrm{H}), 1.23-$ $1.16(\mathrm{~m}, 3 \mathrm{H}), 1.07$ (s, 3H), $1.02-0.99(\mathrm{~m}, 1 \mathrm{H}), 0.97(\mathrm{~s}, 3 \mathrm{H}), 0.95(\mathrm{~d}, J=6.8 \mathrm{~Hz}, 3 \mathrm{H}), 0.85$ (d, $J=6.8 \mathrm{~Hz}, 3 \mathrm{H}), 0.79(\mathrm{~d}, J=6.8 \mathrm{~Hz}, 3 \mathrm{H}), 0.77(\mathrm{~d}, J=6.3 \mathrm{~Hz}, 3 \mathrm{H})$.

${ }^{13} \mathrm{C}-N M R: \quad\left(176 \mathrm{MHz}, \mathrm{CDCl}_{3}\right) ; \delta[\mathrm{ppm}]=215.5,143.5,137.8,70.7,64.6,56.8,55.1,45.3,43.4,41.5$, $39.1,38.0,36.5,35.4,35.0,33.4,32.2,30.6,30.2,29.4,29.2,28.3,24.6,21.6$ (2C), 20.7 , 20.4, 18.2, 15.6.

IR: $\quad\left(\right.$ neat); $\tilde{v}\left[\mathrm{~cm}^{-1}\right]=3411(\mathrm{br} \mathrm{w}), 2952(\mathrm{~m}), 2924(\mathrm{~s}), 2854(\mathrm{~m}), 1738(\mathrm{~m}), 1457(\mathrm{w}), 1376(\mathrm{w})$, $1217(w)$.

HRMS: $\quad$ (ESI-TOF); $\mathrm{m} / \mathrm{z}$ calcd. $\mathrm{C}_{29} \mathrm{H}_{48} \mathrm{O}_{3} \mathrm{Na}^{+}[\mathrm{M}+\mathrm{Na}]^{+}: 467.3496$, found: 467.3488.

Opt. act. $[a]_{D}^{21}=+9.0\left(c=0.18, \mathrm{CHCl}_{3}\right)$.
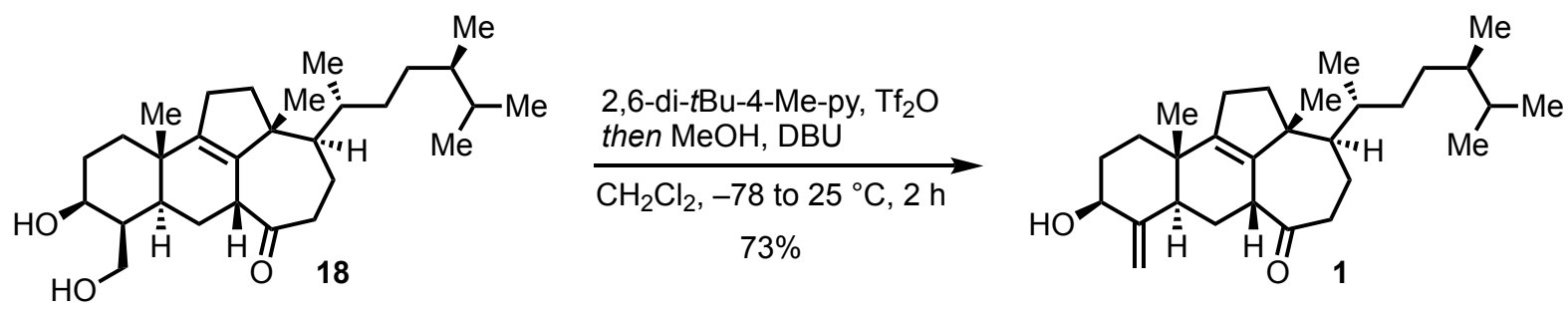

To a solution of diol 18 (4.0 mg, 9.0 $\mu \mathrm{mol}, 1.0 \mathrm{eq})$ in $\mathrm{CH}_{2} \mathrm{Cl}_{2}(1.25 \mathrm{~mL})$ was added 2,6-di-tert-butyl-4-methylpyridine (18.5 mg, $90.0 \mu \mathrm{mol}, 10$ eq.) and it was cooled to $-78{ }^{\circ} \mathrm{C}$. Triflic anhydride $\left(1 \mathrm{M} \mathrm{in} \mathrm{CH}_{2} \mathrm{Cl}_{2}, 23 \mu \mathrm{L}\right.$, $23 \mu \mathrm{mol}, 2.6$ eq.) was added dropwise and the reaction was stirred at this temperature for 5 min before 
$\mathrm{MeOH}(20 \mu \mathrm{L}, 0.50 \mathrm{mmol}, 55$ eq.) and DBU (30 $\mu \mathrm{L}, 0.20 \mu \mathrm{mol}, 22$ eq.) were added. The reaction mixture was allowed to warm to $25^{\circ} \mathrm{C}$ over $1.5 \mathrm{~h}$, and then stirred at this temperature for $1 \mathrm{~h}$ before $\mathrm{HCl}\left(1 \mathrm{M} \mathrm{in} \mathrm{H}_{2} \mathrm{O}\right.$, $5 \mathrm{~mL})$ was added. The aqueous phase was extracted with $\mathrm{CH}_{2} \mathrm{Cl}_{2}(3 \times 10 \mathrm{~mL})$ and the combined organic phases were washed sequentially with $\mathrm{HCl}\left(1 \mathrm{M}\right.$ in $\mathrm{H}_{2} \mathrm{O}, 20 \mathrm{~mL}$ ), $\mathrm{NaHCO}_{3}$ (sat. aq., $20 \mathrm{~mL}$ ), and brine (sat., $20 \mathrm{~mL}$ ), dried over $\mathrm{MgSO}_{4}$, and the solvent was removed under reduced pressure. Column chromatography (silica gel, nhexane/EtOAc 3:1) gave swinhoeisterol A (1) $(2.8 \mathrm{mg}, 6.6 \mu \mathrm{mol}, 73 \%$ ) as an amorphous powder.

TLC: $\quad R_{\mathrm{f}}=0.26($ nhexane/EtOAc 3:1).

1H-NMR: $\quad\left(700 \mathrm{MHz}, \mathrm{CDCl}_{3}\right) ; \delta[\mathrm{ppm}]=5.09(\mathrm{~s}, 1 \mathrm{H}), 4.69(\mathrm{~s}, 1 \mathrm{H}), 4.08-4.00(\mathrm{~m}, 1 \mathrm{H}), 3.17-3.12$ (m, 1H), 2.53 (ddd, $J=16.2,6.1,2.4 \mathrm{~Hz}, 1 \mathrm{H}$ ), 2.36 (ddd, $J=15.9,11.7,3.5 \mathrm{~Hz}, 1 \mathrm{H}$ ), 2.32 $-2.26(\mathrm{~m}, 1 \mathrm{H}), 2.22-2.17(\mathrm{~m}, 1 \mathrm{H}), 2.15(\mathrm{~d}, J=13.1 \mathrm{~Hz}, 1 \mathrm{H}), 2.06-2.02(\mathrm{~m}, 1 \mathrm{H}), 2.00$ $(\mathrm{d}, J=12.6 \mathrm{~Hz}, 1 \mathrm{H}), 1.82-1.78(\mathrm{~m}, 1 \mathrm{H}), 1.78-1.74(\mathrm{~m}, 1 \mathrm{H}), 1.73-1.69(\mathrm{~m}, 1 \mathrm{H}), 1.69-$ $1.63(\mathrm{~m}, 2 \mathrm{H}), 1.60(\mathrm{dt}, J=12.8,6.4 \mathrm{~Hz}, 1 \mathrm{H}), 1.57-1.55(\mathrm{~m}, 2 \mathrm{H}), 1.48$ (qd, $J=12.7,4.3$ $\mathrm{Hz}, 1 \mathrm{H}), 1.42-1.38(\mathrm{~m}, 1 \mathrm{H}), 1.36(\mathrm{td}, J=13.3,4.2 \mathrm{~Hz}, 1 \mathrm{H}), 1.28-1.24(\mathrm{~m}, 1 \mathrm{H}), 1.23-$ $1.16(\mathrm{~m}, 3 \mathrm{H}), 1.01(\mathrm{~s}, 3 \mathrm{H}), 0.93(\mathrm{~d}, J=6.9 \mathrm{~Hz}, 3 \mathrm{H}), 0.92-0.90(\mathrm{~m}, 1 \mathrm{H}), 0.86(\mathrm{~d}, J=6.8$ $\mathrm{Hz}, 3 \mathrm{H}), 0.80$ (d, $J=6.8 \mathrm{~Hz}, 3 \mathrm{H}), 0.78(\mathrm{~d}, J=6.5 \mathrm{~Hz}, 3 \mathrm{H}), 0.78(\mathrm{~s}, 3 \mathrm{H})$.

13C-NMR: $\quad\left(176 \mathrm{MHz}, \mathrm{CDCl}_{3}\right) ; \delta[\mathrm{ppm}]=213.6,151.9,146.9,136.0,102.9,73.1,55.8,53.8,45.4$, $45.3,44.4,39.1,38.7,37.7,35.2,34.8,33.5,32.5,32.2,30.5,27.8,24.5,21.6,20.4,20.1$, 20.0, 18.2, 17.9, 15.6 .

IR: $\quad\left(\right.$ neat); $\tilde{v}\left[\mathrm{~cm}^{-1}\right]=3488(\mathrm{br} w), 2953(\mathrm{~m}), 2922(\mathrm{~s}), 2853(\mathrm{~m}), 1705(\mathrm{w}), 1458(\mathrm{~m}), 1376(\mathrm{w})$, $894(w)$.

HRMS: $\quad$ (ESI-TOF); $\mathrm{m} / \mathrm{z}$ calcd. $\mathrm{C}_{29} \mathrm{H}_{46} \mathrm{O}_{2} \mathrm{Na}^{+}[\mathrm{M}+\mathrm{Na}]^{+}: 449.3390$, found: 449.3400.

Opt. act. $[\mathrm{a}]_{\mathrm{D}}^{20}=+66.8\left(c=0.19, \mathrm{CHCl}_{3}\right)$. 


\section{Attempted Hydrogenation of (22Z)-Diol S19 and NMR Comparison with Epimers S20 and}

S26

(22Z)-13(14 $\rightarrow 8), 14(8 \rightarrow 7)$ diabeo-3a,5-cyclo-5a,7ß(H)-Campesta-8,22-dien-6a,14a-diol (S19)
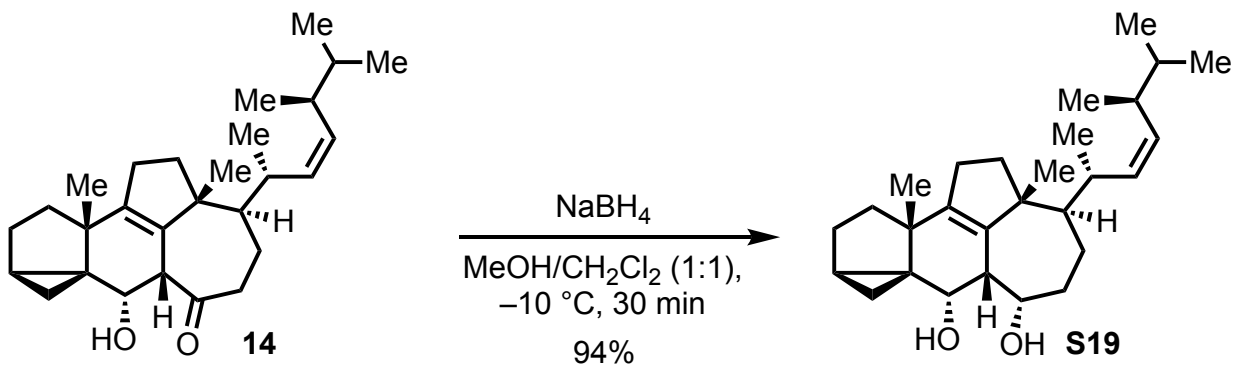

To a solution of 22Z-hydroxy ketone 14 (196 mg, $477 \mu$ mol, 1.0 eq.) in $\mathrm{CH}_{2} \mathrm{Cl}_{2} / \mathrm{MeOH}$ (1:1, $5.0 \mathrm{~mL}$ ) was added $\mathrm{NaBH}_{4}$ ( $45 \mathrm{mg}, 1.2 \mathrm{mmol}, 2.5$ eq.) at $-10^{\circ} \mathrm{C}$. After stirring for $30 \mathrm{~min}$, the reaction mixture was diluted with $\mathrm{CH}_{2} \mathrm{Cl}_{2}(3 \mathrm{~mL})$ and $\mathrm{HCl}\left(1 \mathrm{M}\right.$ in $\left.\mathrm{H}_{2} \mathrm{O}, 5 \mathrm{~mL}\right)$ was added. The aqueous phase was extracted with $\mathrm{CH}_{2} \mathrm{Cl}_{2}$ $(3 \times 10 \mathrm{~mL})$ and the combined organic phases were washed with brine (sat., $20 \mathrm{~mL}$ ), dried over $\mathrm{MgSO}_{4}$, and the solvent was removed under reduced pressure. Column chromatography (silica gel, nhexane/EtOAc 5:1) gave 22Z-diol S19 (185 mg, $448 \mu \mathrm{mol}, 94 \%$ ) as a colorless solid.

$\begin{array}{ll}\text { M.p. } & 195-197^{\circ} \mathrm{C}\left(\mathrm{CHCl}_{3}\right) \\ \text { TLC: } & R_{\mathrm{f}}=0.19(\text { nhexane/EtOAc } 5: 1) .\end{array}$

1H-NMR: $\quad\left(500 \mathrm{MHz}, \mathrm{CDCl}_{3}\right) ; \delta[\mathrm{ppm}]=5.39(\mathrm{t}, J=10.7 \mathrm{~Hz}, 1 \mathrm{H}), 5.07(\mathrm{t}, J=10.7 \mathrm{~Hz}, 1 \mathrm{H}), 4.34-$ $4.23(\mathrm{~m}, 2 \mathrm{H}), 2.78-2.71(\mathrm{~m}, 1 \mathrm{H}), 2.68(\mathrm{dd}, J=6.3,2.3 \mathrm{~Hz}, 1 \mathrm{H}), 2.34-2.20(\mathrm{~m}, 3 \mathrm{H}), 1.98$ $-1.90(\mathrm{~m}, 2 \mathrm{H}), 1.84-1.74(\mathrm{~m}, 2 \mathrm{H}), 1.74-1.68(\mathrm{~m}, 2 \mathrm{H}), 1.66-1.57(\mathrm{~m}, 5 \mathrm{H}), 1.50-1.42$ $(\mathrm{m}, 2 \mathrm{H}), 1.06(\mathrm{~s}, 3 \mathrm{H}), 0.99-0.93(\mathrm{~m}, 9 \mathrm{H}), 0.91(\mathrm{~d}, J=6.7 \mathrm{~Hz}, 3 \mathrm{H}), 0.87(\mathrm{~d}, J=6.8 \mathrm{~Hz}$, $3 \mathrm{H}), 0.71(\mathrm{dd}, J=8.4,5.0 \mathrm{~Hz}, 1 \mathrm{H}), 0.31(\mathrm{t}, J=4.6 \mathrm{~Hz}, 1 \mathrm{H})$.

13C-NMR: $\quad\left(151 \mathrm{MHz}, \mathrm{CDCl}_{3}\right) ; \delta[\mathrm{ppm}]=144.5,140.1,132.8,132.4,66.2,63.7,53.3,51.9,45.4,42.6$, $39.4,38.4,37.3,35.8,33.6,33.2,32.6,28.7,26.1,23.9,22.6,21.0,20.4,20.3,20.3,17.8$, 17.0, 7.1.

IR: $\quad\left(\right.$ neat); $\tilde{v}\left[\mathrm{~cm}^{-1}\right]=3401(\mathrm{w}), 2953(\mathrm{~m}), 2922(\mathrm{~s}), 2852(\mathrm{~m}), 1740(\mathrm{w}), 1459(\mathrm{w}), 1377(\mathrm{w})$, $1244(w), 1169(w), 977(w), 722(w)$.

HRMS: (ESI-TOF); $m / z$ calcd. for $\mathrm{C}_{28} \mathrm{H}_{44} \mathrm{O}_{2} \mathrm{Na}^{+}[\mathrm{M}+\mathrm{Na}]+:$ 435.3234, found: 435.3245 . 


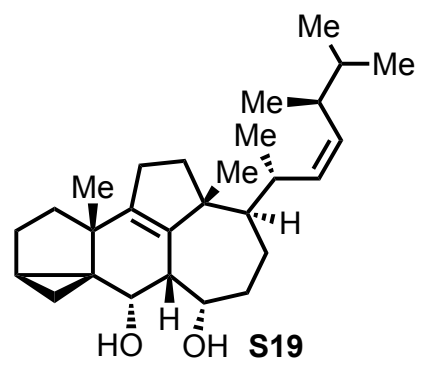

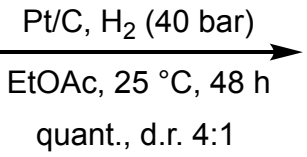

quant., d.r. $4: 1$

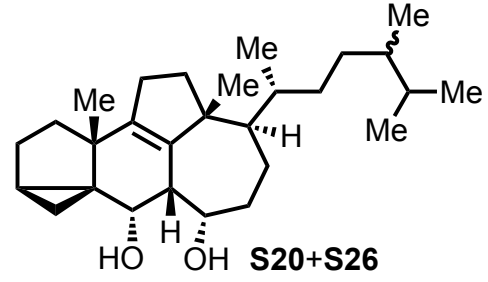

To a suspension of 22Z-diol S19 (13.5 mg, $32.8 \mu \mathrm{mol} 1.0$ eq.) in EtOAc (1 mL) in a Parr reactor was added $\mathrm{Pt} / \mathrm{C}(10 \% w / w, 6.4 \mathrm{mg}, 3.3 \mu \mathrm{mol}, 0.1$ eq.). The reactor was purged with hydrogen gas three times before stirring the reaction mixture at $25^{\circ} \mathrm{C}$ under 40 bar of hydrogen atmosphere for $48 \mathrm{~h}$. The reactor was opened carefully, the reaction mixture was filtered through Celite ${ }^{\circledR}$, rinsed with EtOAc $(3 \times 10 \mathrm{~mL})$, and the solvent was removed under reduced pressure to give $\mathbf{S} 20$ and $\mathbf{S 2 6}$ as inseparable mixture of epimers (d.r. 4:1, determined by integration of C24' signals $\delta$ [ppm] = 18.2 (major, S20) and 17.9 (minor, S26) in ${ }^{13} \mathrm{C}-\mathrm{NMR}$ of the crude product) as a colorless solid.

TLC: $\quad R_{\mathrm{f}}=0.20($ nhexane/EtOAc 5:1).

1H-NMR: $\quad\left(500 \mathrm{MHz}, \mathrm{CDCl}_{3}\right) ; \delta[\mathrm{ppm}]=4.27(\mathrm{~d}, J=6.3 \mathrm{~Hz}, 1 \mathrm{H}), 4.22(\mathrm{~d}, J=6.3 \mathrm{~Hz}, 1 \mathrm{H}), 2.69(\mathrm{~d}, J=$ $6.0 \mathrm{~Hz}, 1 \mathrm{H}), 2.30-2.16(\mathrm{~m}, 2 \mathrm{H}), 1.94-1.86(\mathrm{~m}, 2 \mathrm{H}), 1.80-1.74(\mathrm{~m}, 1 \mathrm{H}), 1.71-1.66(\mathrm{~m}$, $1 \mathrm{H}), 1.64-1.60(\mathrm{~m}, 2 \mathrm{H}), 1.60-1.49(\mathrm{~m}, 4 \mathrm{H}), 1.47-1.42(\mathrm{~m}, 2 \mathrm{H}), 1.40-1.33(\mathrm{~m}, 1 \mathrm{H})$, $1.23-1.14(\mathrm{~m}, 2 \mathrm{H}), 1.10-1.06(\mathrm{~m}, 1 \mathrm{H}), 1.05(\mathrm{~s}, 3 \mathrm{H}), 1.03-0.97(\mathrm{~m}, 1 \mathrm{H}), 0.96(\mathrm{~s}, 3 \mathrm{H})$, $0.89(\mathrm{~d}, J=6.8 \mathrm{~Hz}, 3 \mathrm{H}), 0.85(\mathrm{~d}, J=6.8 \mathrm{~Hz}, 3 \mathrm{H}), 0.79(\mathrm{~d}, J=6.8 \mathrm{~Hz}, 6 \mathrm{H}), 0.78(\mathrm{~d}, J=6.6$ $\mathrm{Hz}, 3 \mathrm{H}), 0.69$ (dd, $J=8.4,5.0 \mathrm{~Hz}, 1 \mathrm{H}), 0.28(\mathrm{t}, J=4.6 \mathrm{~Hz}, 1 \mathrm{H})$.

13C-NMR: $\quad\left(126 \mathrm{MHz}, \mathrm{CDCl}_{3}\right) ; \delta[\mathrm{ppm}]=144.7,140.1,66.5,64.3,53.6,52.3,45.5,42.5,39.6,(39.2)^{\star}$, $39.1,37.5,35.8,(35.2)^{\star}, 34.9,(33.8)^{*}, 33.6,33.2,32.2,(31.8)^{\star},(30.4)^{\star}, 30.3,28.5,26.1$, $23.9,(22.0)^{\star}, 21.9,20.8,20.5,20.4,18.4,18.2,(17.9)^{\star}, 15.6,7.2 .{ }^{*}$ minor epimer $\mathbf{S} 26$

IR: $\quad\left(\right.$ neat); $\tilde{v}\left[\mathrm{~cm}^{-1}\right]=3382(\mathrm{br} \mathrm{w}), 2953(\mathrm{~m}), 2924(\mathrm{~s}), 2854(\mathrm{~m}), 1735(\mathrm{w}), 1459(\mathrm{w}), 1376(\mathrm{w})$, $1215(\mathrm{w}), 760(\mathrm{~m})$.

HRMS: $\quad$ (ESI-TOF); $m / z$ calcd. for $\mathrm{C}_{28} \mathrm{H}_{46} \mathrm{O}_{2} \mathrm{Na}^{+}[\mathrm{M}+\mathrm{Na}]+:$ 437.3390, found: 437.3404 . 

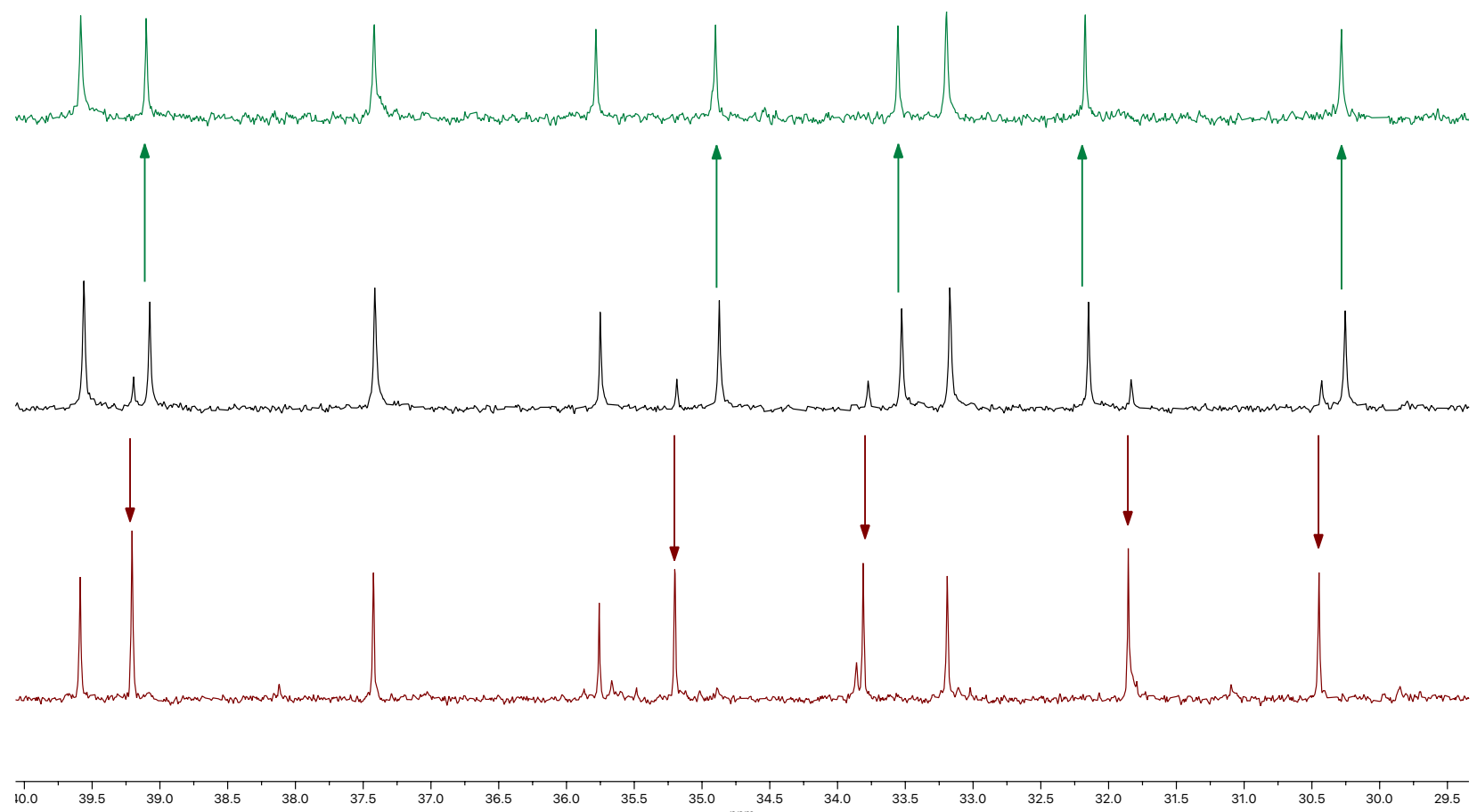

${ }^{13} \mathrm{C}$-NMR Comparison of campestane S20 (green), epimeric mixture (black) and ergostane S26 (red), (shifts in ppm).

$\begin{array}{lllllllllllllll}\text { S20 } & 39.6 & & 39.1 & 37.4 & 35.8 & & 34.9 & & 33.6 & 33.2 & 32.2 & & & 30.3 \\ \text { mix } & 39.6 & 39.2 & 39.1 & 37.4 & 35.8 & 35.2 & 34.9 & 33.8 & 33.5 & 33.2 & 32.1 & 31.8 & 30.4 & 30.3 \\ \text { S26 } & 39.6 & 39.2 & & 37.4 & 35.8 & 35.2 & & 33.8 & & 33.2 & & 31.9 & 30.4 & \end{array}$

Campestane diol $\mathbf{S 2 0}$ was synthesized to enable the assignment of signals in the ${ }^{13} \mathrm{C}-\mathrm{NMR}$ spectrum of the epimeric mixture, for analytical data of ergostane diol S26 see page S49-50.

13(14 $\rightarrow 8), 14(8 \rightarrow 7)$ diabeo-3a,5-cyclo-5a,7ß(H)-Campesta-8-en-6a,14a-diol (S20)
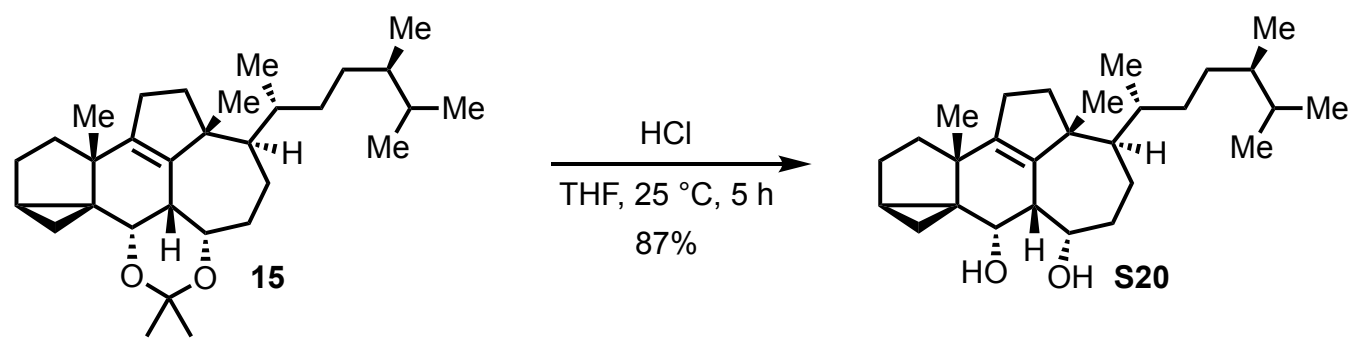

Acetonide 15 was dissolved in THF ( $1 \mathrm{~mL}), \mathrm{HCl}\left(1 \mathrm{~m}\right.$ in $\left.\mathrm{H}_{2} \mathrm{O}, 1 \mathrm{~mL}\right)$ was added and the reaction mixture was stirred at $25^{\circ} \mathrm{C}$ for $5 \mathrm{~h}$. It was diluted with EtOAc $(3 \mathrm{~mL})$ and $\mathrm{NaHCO}_{3}$ (sat. aq., $5 \mathrm{~mL}$ ) was added carefully. The aqueous phase was extracted with EtOAc $(3 \times 10 \mathrm{~mL})$, and the combined organic phases 
were washed with brine (sat., $20 \mathrm{~mL}$ ), dried over $\mathrm{MgSO}_{4}$, and the solvent was removed under reduced pressure. Column chromatography (silica gel, nhexane/EtOAc 5:1) gave diol $\mathbf{S 2 0}(87 \%)$ as a colorless solid.

M.p. $\quad 147-149^{\circ} \mathrm{C}\left(\mathrm{CHCl}_{3}\right)$

TLC: $\quad R_{\mathrm{f}}=0.27($ nhexane/EtOAc $4: 1)$.

1H-NMR: $\quad\left(500 \mathrm{MHz}, \mathrm{CDCl}_{3}\right) ; \delta[\mathrm{ppm}]=4.26(\mathrm{~d}, J=6.2 \mathrm{~Hz}, 1 \mathrm{H}), 4.22(\mathrm{~d}, J=6.1 \mathrm{~Hz}, 1 \mathrm{H}), 2.69(\mathrm{~d}, J=$ $6.0 \mathrm{~Hz}, 1 \mathrm{H}), 2.29-2.16(\mathrm{~m}, 2 \mathrm{H}), 1.93-1.85(\mathrm{~m}, 2 \mathrm{H}), 1.79-1.73(\mathrm{~m}, 1 \mathrm{H}), 1.72-1.65(\mathrm{~m}$, $2 \mathrm{H}), 1.65-1.58(\mathrm{~m}, 3 \mathrm{H}), 1.58-1.50(\mathrm{~m}, 4 \mathrm{H}), 1.48-1.40(\mathrm{~m}, 3 \mathrm{H}), 1.40-1.31(\mathrm{~m}, 1 \mathrm{H})$, $1.20-1.14(\mathrm{~m}, 2 \mathrm{H}), 1.09-1.05(\mathrm{~m}, 1 \mathrm{H}), 1.04(\mathrm{~d}, J=2.3 \mathrm{~Hz}, 3 \mathrm{H}), 0.95(\mathrm{~s}, 3 \mathrm{H}), 0.88$ (d, J $=6.8 \mathrm{~Hz}, 3 \mathrm{H}), 0.85(\mathrm{~d}, J=6.8 \mathrm{~Hz}, 3 \mathrm{H}), 0.79(\mathrm{~d}, J=6.9 \mathrm{~Hz}, 3 \mathrm{H}), 0.77(\mathrm{~d}, J=6.6 \mathrm{~Hz}, 3 \mathrm{H})$, 0.69 (dd, $J=8.4,5.0 \mathrm{~Hz}, 1 \mathrm{H}), 0.28(\mathrm{t}, J=4.6 \mathrm{~Hz}, 1 \mathrm{H})$.

13C-NMR: $\quad\left(126 \mathrm{MHz}, \mathrm{CDCl}_{3}\right) ; \delta[\mathrm{ppm}]=144.7,140.1,66.4,64.4,53.6,52.3,45.5,42.5,39.6,39.1$, $37.4,35.8,34.9,33.6,33.2,32.2,30.3,28.5,26.1,23.9,21.8,20.8,20.5,20.4,18.4,18.1$, 15.6, 7.1.

IR: $\quad\left(\right.$ neat); $\tilde{v}\left[\mathrm{~cm}^{-1}\right]=3393(\mathrm{br} \mathrm{w}), 2953(\mathrm{~s}), 2924(\mathrm{~s}), 2854(\mathrm{~m}), 1739(\mathrm{w}), 1456(\mathrm{~m}), 1409(\mathrm{~m})$, $1370(\mathrm{~m}), 1204(\mathrm{w}), 1073(\mathrm{~m}), 1044(\mathrm{w}), 946(\mathrm{w}), 759(\mathrm{w})$.

HRMS: (ESI-TOF); $m / z$ calcd. for $\mathrm{C}_{28} \mathrm{H}_{46} \mathrm{O}_{2} \mathrm{Na}^{+}[\mathrm{M}+\mathrm{Na}]^{+}: 437.3390$, found: 437.3378

Opt. act. $[\mathrm{a}]_{\mathrm{D}}^{22}=+95.0\left(c=1.00, \mathrm{CHCl}_{3}\right)$.

\section{Attempted late-stage Ozonolysis and Julia-Kocienski Olefination of Enone 16}

3,14-Dioxo-13(14 $\rightarrow 8), 14(8 \rightarrow 7)$ diabeo-7 $\beta(H)$-pregn-4,8-dien-20a-carboxaldehyde (S21)
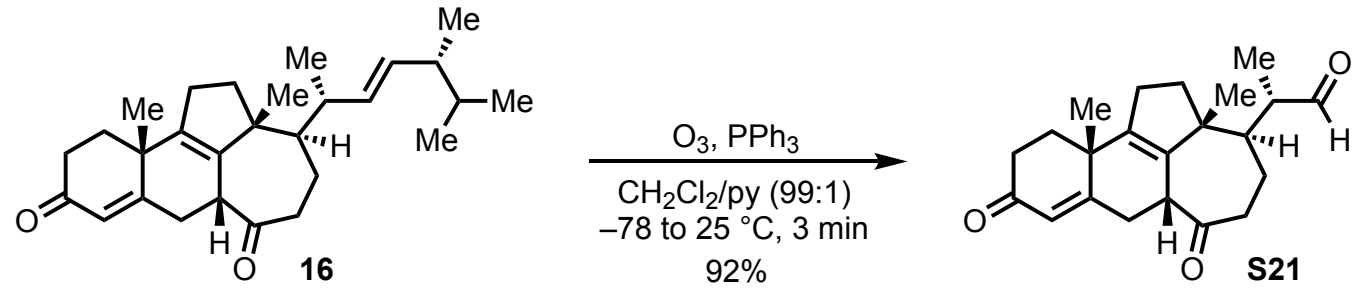

A stream of ozone-rich oxygen was passed through a solution of enone 16 (13.5 mg, $33.0 \mu \mathrm{mol}, 1.0 \mathrm{eq}$.) in $\mathrm{CH}_{2} \mathrm{Cl}_{2}$ /pyridine $(99: 1,1 \mathrm{~mL})$ at $-78{ }^{\circ} \mathrm{C}$ for $3 \mathrm{~min}$. $\mathrm{PPh}_{3}(9.5 \mathrm{mg}, 36 \mu \mathrm{mol}, 1.1$ eq.) was added and the reaction mixture was allowed to warm to $25^{\circ} \mathrm{C}$ over $16 \mathrm{~h}$. The solvent was removed, the residue was adsorbed on silica gel and column chromatography (silica gel, nhexane/EtOAc 1:1) gave aldehyde S21 (10 mg, $30 \mu \mathrm{mol}, 92 \%$ ) as a colorless oil.

TLC: $\quad R_{\mathrm{f}}=0.30($ nhexane/EtOAc $1: 1)$. 
1H-NMR: $\quad\left(700 \mathrm{MHz}, \mathrm{CDCl}_{3}\right) ; \delta[\mathrm{ppm}]=9.79(\mathrm{~d}, J=2.5 \mathrm{~Hz}, 1 \mathrm{H}), 5.90(\mathrm{~s}, 1 \mathrm{H}), 3.31-3.29(\mathrm{~m}, 1 \mathrm{H})$, $2.89(\mathrm{dd}, J=13.2,1.5 \mathrm{~Hz}, 1 \mathrm{H}), 2.70-2.66(\mathrm{~m}, 1 \mathrm{H}), 2.57-2.51(\mathrm{~m}, 2 \mathrm{H}), 2.49$ (ddd, $J=$ $13.1,6.8,1.8 \mathrm{~Hz}, 1 \mathrm{H}), 2.44-2.41(\mathrm{~m}, 1 \mathrm{H}), 2.41-2.38(\mathrm{~m}, 1 \mathrm{H}), 2.38-2.34(\mathrm{~m}, 2 \mathrm{H}), 2.20$ $-2.15(\mathrm{~m}, 1 \mathrm{H}), 1.99$ (ddd, $J=13.2,5.2,2.2 \mathrm{~Hz}, 1 \mathrm{H}), 1.93$ (dd, $J=8.1,5.5 \mathrm{~Hz}, 1 \mathrm{H}), 1.91-$ $1.88(\mathrm{~m}, 1 \mathrm{H}), 1.80-1.76(\mathrm{~m}, 1 \mathrm{H}), 1.73-1.70(\mathrm{~m}, 1 \mathrm{H}), 1.54(\mathrm{dt}, J=11.3,2.1 \mathrm{~Hz}, 1 \mathrm{H}), 1.30$ (s, 3H), $1.18(\mathrm{~s}, 3 \mathrm{H}), 1.16(\mathrm{~d}, J=7.0 \mathrm{~Hz}, 3 \mathrm{H})$.

13C-NMR: $\quad\left(176 \mathrm{MHz}, \mathrm{CDCl}_{3}\right) ; \delta[\mathrm{ppm}]=209.6,205.0,198.4,166.2,144.5,136.3,126.4,55.0,53.0$, 48.4, 46.0, 44.8, 38.2, 37.6, 34.1, 33.6, 32.4, 27.4, 22.4, 21.2, 21.1, 15.7.

IR: $\quad\left(\right.$ neat); $\tilde{v}\left[\mathrm{~cm}^{-1}\right]=2958(\mathrm{~m}), 2924(\mathrm{~s}), 2853(\mathrm{~m}), 1708(\mathrm{~s}), 1667(\mathrm{~s}), 1458(\mathrm{~m}), 1377(\mathrm{w})$, $1238(w), 1186(w), 870(w)$.

HRMS: (ESI-TOF); $m / z$ calcd. for $\mathrm{C}_{22} \mathrm{H}_{28} \mathrm{O}_{3} \mathrm{Na}^{+}[\mathrm{M}+\mathrm{Na}]^{+}:$363.1931, found: 363.1934.

Opt. act. $\quad[a]_{D}^{27}=+131.0\left(c=1.00, \mathrm{CHCl}_{3}\right)$.

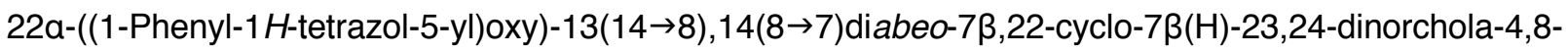
dien-3,14-dione (S22)
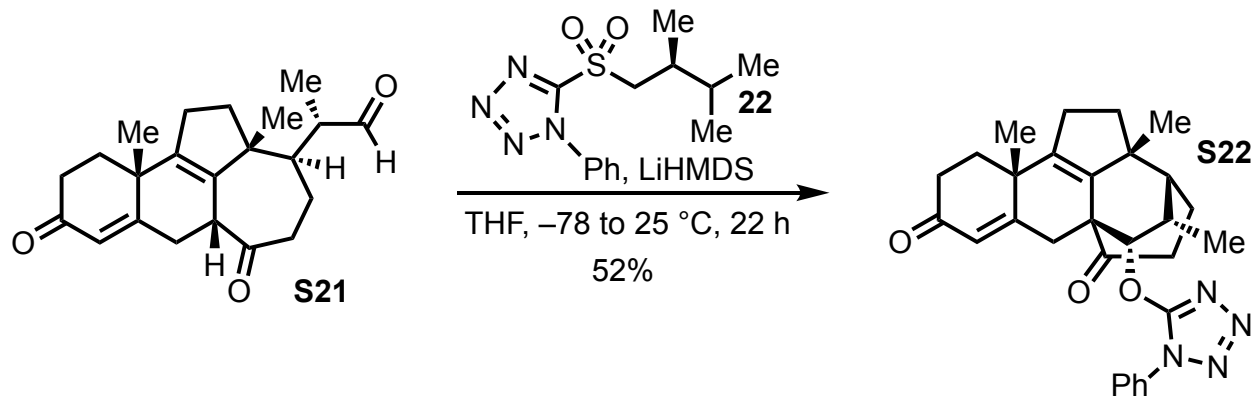

To a solution of hexamethyldisilazane $(15 \mu \mathrm{L}, 74 \mathrm{mmol}, 5$ eq. $)$ in THF $(0.2 \mathrm{~mL})$ at $0{ }^{\circ} \mathrm{C}$ was added $n B u L i$ (1.6 $\mathrm{M}$ in nhexane, $46 \mu \mathrm{L}, 74 \mu \mathrm{mol}, 5$ eq.) and it was stirred for $15 \mathrm{~min}$ at this temperature before the solution was added to sulfone 22 ( $21 \mathrm{mg}, 74 \mu \mathrm{mol}, 5.0$ eq.) in THF $(1 \mathrm{~mL})$ at $-78^{\circ} \mathrm{C}$. The resulting bright yellow solution was stirred at $-65^{\circ} \mathrm{C}$ for $1 \mathrm{~h}$ and a solution of aldehyde S21 ( $5 \mathrm{mg}, 15 \mu \mathrm{mol}, 1.0 \mathrm{eq}$.) in THF $(0.5 \mathrm{~mL})$ was added to the reaction mixture over $10 \mathrm{~min}$, and the reaction mixture was allowed to warm to $25^{\circ} \mathrm{C}$ over $20 \mathrm{~h}$. EtOAc $(3 \mathrm{~mL})$ and $\mathrm{H}_{2} \mathrm{O}(5 \mathrm{~mL})$ were added to the reaction mixture and the aqueous phase was extracted with EtOAc $(3 \times 10 \mathrm{~mL})$. The combined organic phases were washed sequentially with $\mathrm{NaHCO}_{3}$ (sat. aq., $20 \mathrm{~mL}$ ) and brine (sat., $20 \mathrm{~mL}$ ), dried over $\mathrm{MgSO}_{4}$, and the solvent was removed under reduced pressure. Column chromatography (silica gel, nhexane/EtOAc 4:1) gave dione S22 (3.8 mg, $7.8 \mu \mathrm{mol}, 52 \%)$ as a colorless oil.

TLC: $\quad R_{\mathrm{f}}=0.35($ nhexane/EtOAc $1: 1)$. 
1H-NMR: $\quad\left(700 \mathrm{MHz}, \mathrm{CDCl}_{3}\right) ; \delta[\mathrm{ppm}]=7.94-7.91(\mathrm{~m}, 2 \mathrm{H}), 7.61-7.58(\mathrm{~m}, 2 \mathrm{H}), 7.48-7.45(\mathrm{~m}, 1 \mathrm{H})$, $5.77(\mathrm{~s}, 1 \mathrm{H}), 5.41(\mathrm{~d}, J=9.7 \mathrm{~Hz}, 1 \mathrm{H}), 3.37-3.30(\mathrm{~m}, 1 \mathrm{H}), 3.10(\mathrm{~d}, J=12.7 \mathrm{~Hz}, 1 \mathrm{H}), 2.76$ $-2.67(\mathrm{~m}, 2 \mathrm{H}), 2.59-2.52(\mathrm{~m}, 1 \mathrm{H}), 2.47(\mathrm{dd}, J=12.7,1.8 \mathrm{~Hz}, 1 \mathrm{H}), 2.46-2.41(\mathrm{~m}, 1 \mathrm{H})$, 2.31 (dd, $J=15.9,8.4 \mathrm{~Hz}, 1 \mathrm{H}$ ), 2.24 (ddd, $J=14.8,7.3,2.7 \mathrm{~Hz}, 1 \mathrm{H}$ ), 2.19 (dt, $J=6.8,3.6$ $\mathrm{Hz}, 1 \mathrm{H}), 2.16-2.11(\mathrm{~m}, 1 \mathrm{H}), 2.05-2.01(\mathrm{~m}, 1 \mathrm{H}), 1.94-1.89(\mathrm{~m}, 2 \mathrm{H}), 1.81(\mathrm{dd}, J=11.8$, $5.8 \mathrm{~Hz}, 1 \mathrm{H}), 1.75(\mathrm{td}, J=13.9,4.9 \mathrm{~Hz}, 1 \mathrm{H}), 1.31(\mathrm{~s}, 3 \mathrm{H}), 1.27(\mathrm{~s}, 3 \mathrm{H}), 0.82(\mathrm{~d}, J=7.6 \mathrm{~Hz}$, $3 \mathrm{H})$.

13C-NMR: $\quad\left(176 \mathrm{MHz}, \mathrm{CDCl}_{3}\right) ; \delta[\mathrm{ppm}]=208.8,198.3,164.4,160.1,142.0,142.0,133.7,129.9,128.9$, $128.3,120.6,83.9,56.4,48.0,44.4,38.9,38.2,37.7,37.1,34.2,32.7,31.7,28.0,24.3$, 23.3, 18.5, 14.2.

HRMS: $\quad$ (ESI-TOF); $m / z$ calcd. for $\mathrm{C}_{29} \mathrm{H}_{32} \mathrm{~N}_{4} \mathrm{O}_{3} \mathrm{Na}^{+}[\mathrm{M}+\mathrm{Na}]^{+}: 507.2367$, found: 507.2375. 


\subsection{Synthesis of 24-epi-Swinhoeisterol A (24-epi-1)}

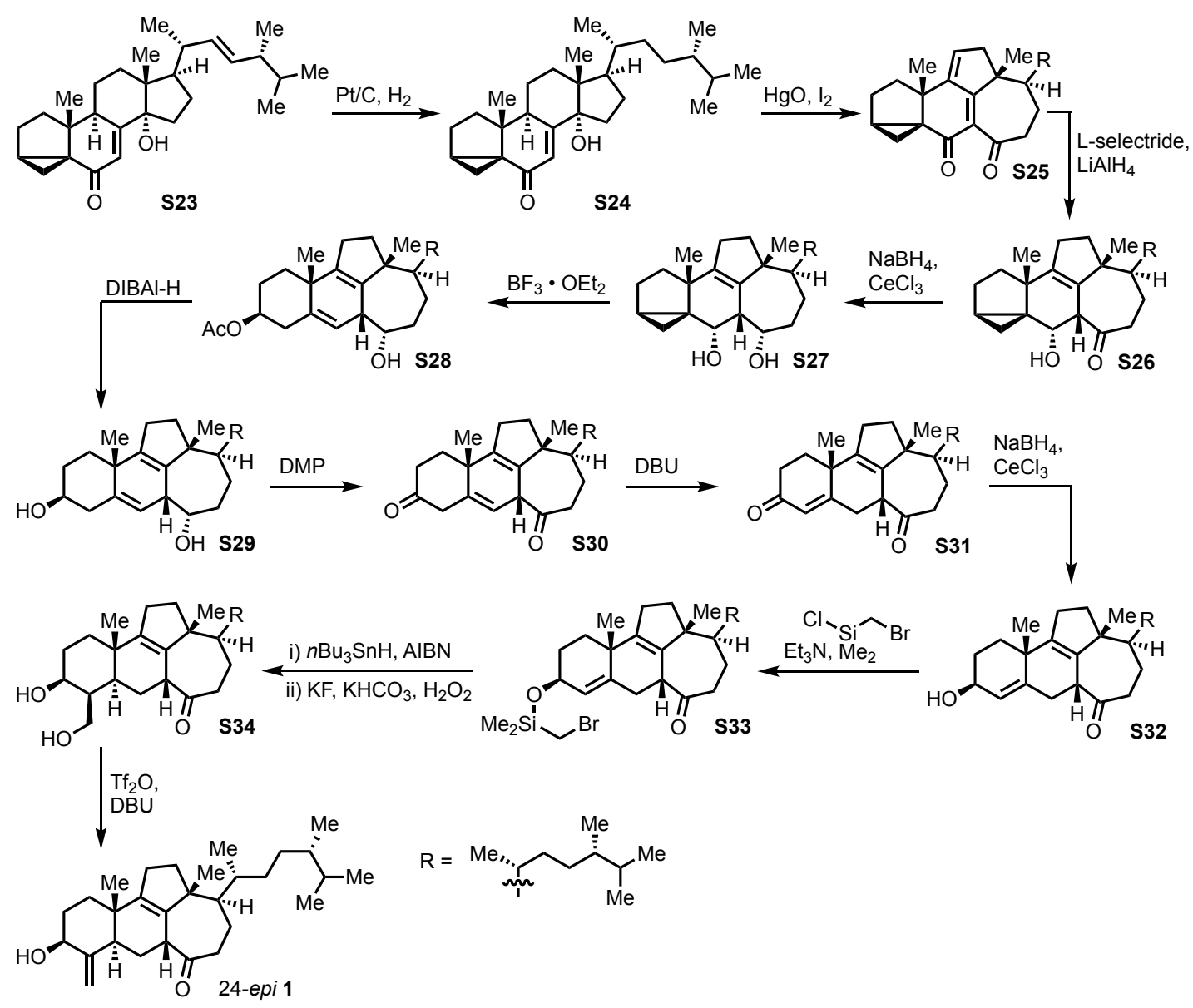

14-Hydroxy-3a,5-cyclo-5a-ergosta-7-en-6-one (S23)

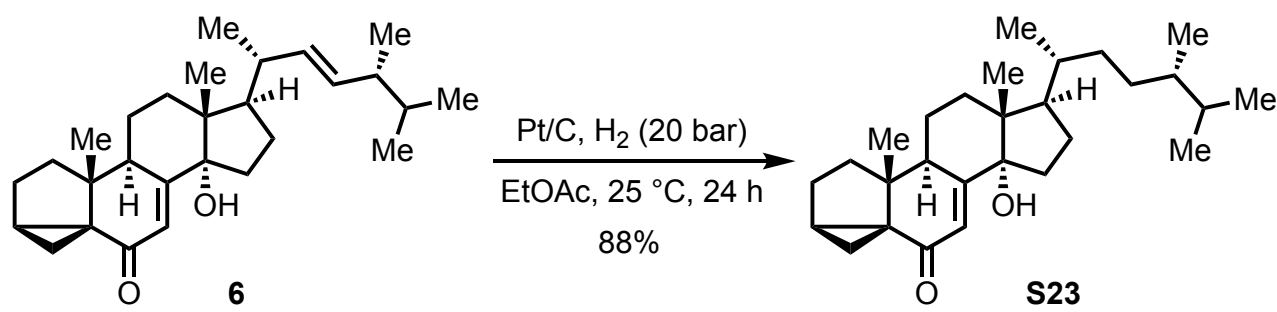

To a solution of $\mathrm{y}$-hydroxy enone $6(3.90 \mathrm{~g}, 9.50 \mathrm{mmol}, 1.0$ eq.) in EtOAc $(95 \mathrm{~mL})$ in a Parr reactor was added Pt/C (10\% w/w, $926 \mathrm{mg}, 475 \mu \mathrm{mol}, 0.05$ eq.). The reactor was purged with hydrogen gas three times before stirring the reaction mixture at $25^{\circ} \mathrm{C}$ under 20 bar of hydrogen atmosphere for $24 \mathrm{~h}$. The reactor was opened carefully, the reaction mixture was filtered through Celite ${ }^{\circledR}$, rinsed with EtOAc $(100 \mathrm{~mL})$, and the solvent was removed under reduced pressure. The crude product was adsorbed on silica gel and column 
chromatography (silica gel, $\mathrm{n}$ hexane/EtOAc 5:1) gave $\mathrm{\gamma}$-hydroxy enone $\mathbf{S} 23$ (3.44 g, $8.33 \mathrm{mmol}, 88 \%$ ) as a colorless solid.
M.p.: $\quad 194-198^{\circ} \mathrm{C}(\mathrm{EtOAC})$.
TLC: $\quad R_{\mathrm{f}}=0.40($ nhexane/EtOAc 3:1).
1H-NMR: $\quad\left(500 \mathrm{MHz}, \mathrm{CDCl}_{3}\right) ; \delta[\mathrm{ppm}]=5.97(\mathrm{~d}, J=2.6 \mathrm{~Hz}, 1 \mathrm{H}), 2.76(\mathrm{ddd}, J=10.7,7.6,2.7 \mathrm{~Hz}, 1 \mathrm{H})$, $2.14-1.98(\mathrm{~m}, 3 \mathrm{H}), 1.98-1.90(\mathrm{~m}, 2 \mathrm{H}), 1.80(\mathrm{dt}, J=8.8,4.6 \mathrm{~Hz}, 1 \mathrm{H}), 1.77-1.64(\mathrm{~m}$, $6 \mathrm{H}), 1.63-1.55(\mathrm{~m}, 2 \mathrm{H}), 1.50-1.36(\mathrm{~m}, 5 \mathrm{H}), 1.27-1.19(\mathrm{~m}, 1 \mathrm{H}), 1.16-1.11(\mathrm{~m}, 1 \mathrm{H})$, $1.09(\mathrm{~s}, 3 \mathrm{H}), 1.03-0.96(\mathrm{~m}, 2 \mathrm{H}), 0.94(\mathrm{~d}, J=6.5 \mathrm{~Hz}, 3 \mathrm{H}), 0.86(\mathrm{~d}, J=6.8 \mathrm{~Hz}, 3 \mathrm{H}), 0.81-$ $0.76(\mathrm{~m}, 7 \mathrm{H}), 0.75(\mathrm{~s}, 3 \mathrm{H})$.

13C-NMR: $\quad\left(101 \mathrm{MHz}, \mathrm{CDCl}_{3}\right) ; \delta[\mathrm{ppm}]=198.4,164.4,123.4,85.4,50.6,46.5,45.3,45.3,44.0,39.1$, $37.9,36.2,35.6,33.9,33.7,31.6,31.0,30.7,26.9,26.7,21.9,20.7,19.4,19.2,17.7,16.1$, 15.5, 13.8.

IR: $\quad$ (neat); $\tilde{v}\left[\mathrm{~cm}^{-1}\right]=3430(\mathrm{br} \mathrm{w}), 2956(\mathrm{~s}), 2929(\mathrm{~s}), 2870(\mathrm{~m}), 1648(\mathrm{~s}), 1457(\mathrm{w}), 1377(\mathrm{~m})$, $1316(m), 1174(m), 1144(w), 888(w)$.

HRMS: (ESI-TOF) $\mathrm{m} / \mathrm{z}$ calcd. for $\mathrm{C}_{28} \mathrm{H}_{44} \mathrm{O}_{2} \mathrm{Na}^{+}[\mathrm{M}+\mathrm{Na}]^{+}:$435.3234, found: 435.3256.

Opt. act. $\quad[a]_{D}^{20}=+110.6\left(c=1.00, \mathrm{CHCl}_{3}\right)$.

13(14 $\rightarrow 8), 14(8 \rightarrow 7)$ Diabeo-3a,5-cyclo-5a-ergosta-7,9(11)-dien-6,14-dione (S24)
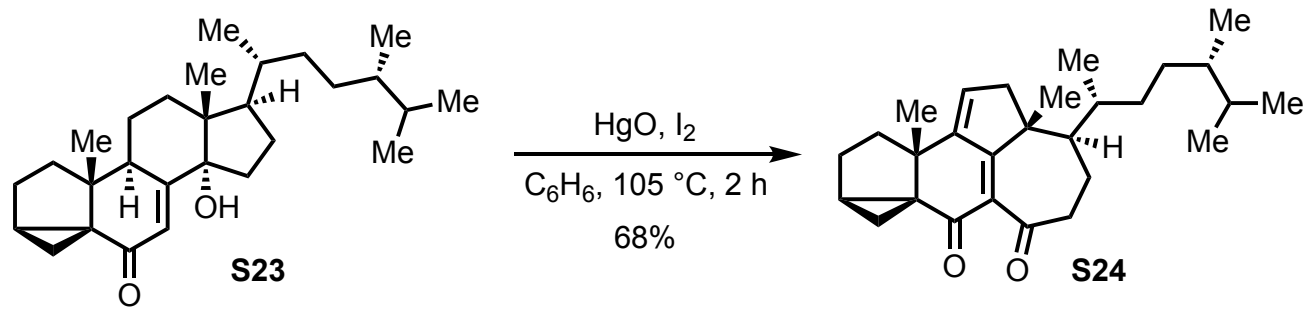

Through a solution of $\mathrm{Y}$-hydroxy enone $\mathbf{S} 23(2.70 \mathrm{~g}, 6.54 \mathrm{mmol}, 1.0 \mathrm{eq}$.) in benzene (270 $\mathrm{mL})$ in a pressure vessel at $60^{\circ} \mathrm{C}$ was bubbled argon via cannula for $15 \mathrm{~min}$. After addition of iodine $(3.98 \mathrm{~g}, 15.7 \mathrm{mmol}$, 2.4 eq.) and $\mathrm{HgO}$ (yellow, $3.82 \mathrm{~g}, 17.6 \mathrm{mmol}, 2.7$ eq.) at the same temperature, the tube was sealed, transferred to a preheated oil bath, and the reaction was stirred at $105^{\circ} \mathrm{C}$ for $2 \mathrm{~h}$. After cooling to $25^{\circ} \mathrm{C}$, the mixture was filtered through Celite ${ }^{\circledR}$, and was rinsed with EtOAc $(100 \mathrm{~mL})$. The organic phase was washed sequentially with $\mathrm{Na}_{2} \mathrm{~S}_{2} \mathrm{O}_{3}$ (sat. aq., $400 \mathrm{~mL}$ ) and brine (sat., $400 \mathrm{~mL}$ ), dried over $\mathrm{MgSO}_{4}$, and the solvent was removed under reduced pressure. Column chromatography (silica gel, nhexane/EtOAc 5:1 $\rightarrow$ 3:1) gave diene dione S24 (1.82 g, $4.45 \mathrm{mmol}, 68 \%)$ as a light-yellow foam.

TLC: $\quad R_{\mathrm{f}}=0.33($ nhexane/EtOAc 3:1). 
1H-NMR: $\quad\left(500 \mathrm{MHz}, \mathrm{CDCl}_{3}\right) ; \delta[\mathrm{ppm}]=6.32(\mathrm{t}, J=2.9 \mathrm{~Hz}, 1 \mathrm{H}), 2.74-2.65(\mathrm{~m}, 2 \mathrm{H}), 2.59(\mathrm{td}, J=$ 11.4, $5.0 \mathrm{~Hz}, 1 \mathrm{H}$ ), 2.41 (dd, $J=17.8,3.3 \mathrm{~Hz}, 1 \mathrm{H}$ ), 2.07 (dd, $J=13.6,7.8 \mathrm{~Hz}, 1 \mathrm{H}$ ), $1.95-$ $1.89(\mathrm{~m}, 2 \mathrm{H}), 1.89-1.81(\mathrm{~m}, 2 \mathrm{H}), 1.78(\mathrm{dd}, J=10.2,4.2 \mathrm{~Hz}, 1 \mathrm{H}), 1.69(\mathrm{dd}, J=12.8,7.5$ $\mathrm{Hz}, 1 \mathrm{H}), 1.65-1.61(\mathrm{~m}, 1 \mathrm{H}), 1.58-1.53(\mathrm{~m}, 2 \mathrm{H}), 1.48-1.37(\mathrm{~m}, 3 \mathrm{H}), 1.17(\mathrm{~s}, 3 \mathrm{H}), 1.15$ (s, 3H), $0.99(\mathrm{~d}, J=6.9 \mathrm{~Hz}, 3 \mathrm{H}), 0.95(\mathrm{q}, J=3.8 \mathrm{~Hz}, 1 \mathrm{H}), 0.93-0.89(\mathrm{~m}, 2 \mathrm{H}), 0.85(\mathrm{~d}, J=$ $6.8 \mathrm{~Hz}, 3 \mathrm{H}), 0.80-0.76(\mathrm{~m}, 7 \mathrm{H})$.

${ }^{13} \mathrm{C}-\mathrm{NMR}: \quad\left(126 \mathrm{MHz}, \mathrm{CDCl}_{3}\right) ; \delta[\mathrm{ppm}]=204.5,193.0,167.7,145.8,136.6,131.2,53.5,50.5,48.5$, $45.5,43.5,41.1,39.1,36.3,36.1,35.4,33.6,31.8,30.5,26.4,26.3,23.6,22.6,21.4,20.5$, 17.8, 15.6, 12.9.

IR: $\quad\left(\right.$ neat); $\tilde{v}\left[\mathrm{~cm}^{-1}\right]=2954(\mathrm{~s}), 2924(\mathrm{~s}), 2854(\mathrm{~m}), 1732(\mathrm{w}), 1704(\mathrm{~m}), 1648(\mathrm{w}), 1458(\mathrm{w})$, 1377 (w).

HRMS: $\quad$ (ESI-TOF); $m / z$ calcd. for $\mathrm{C}_{28} \mathrm{H}_{40} \mathrm{O}_{2} \mathrm{Na}^{+}[\mathrm{M}+\mathrm{Na}]^{+}: 431.2921$, found: 431.2930 .

Opt. act. $[a]_{D}^{23}=-8.4\left(c=1.01, \mathrm{CH}_{2} \mathrm{Cl}_{2}\right)$.

6a-Hydroxy-13(14 $\rightarrow 8), 14(8 \rightarrow 7)$ diabeo-3a,5-cyclo-5a,7ß(H)-ergosta-8-en-14-one (S25)
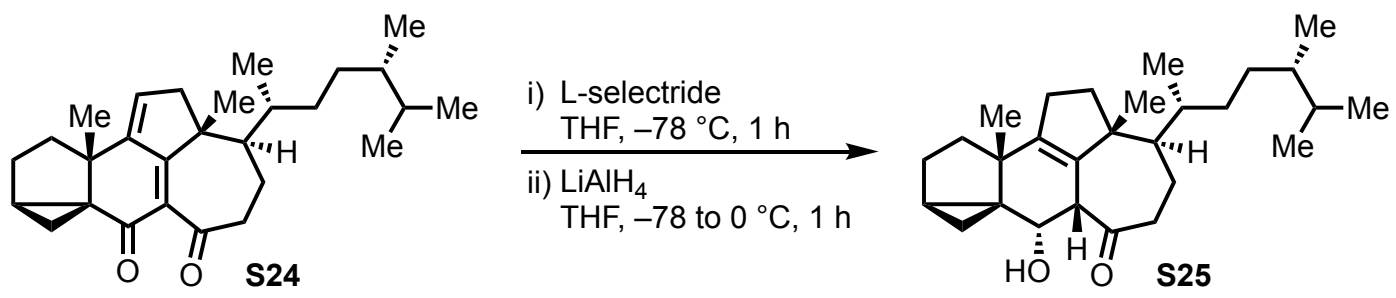

A solution of diene dione $\mathbf{S 2 4}$ (625 mg, $1.53 \mathrm{mmol}, 1.0$ eq.) in THF (15 mL) was degassed applying three freeze-pump-thaw cycles. After cooling to $-78{ }^{\circ} \mathrm{C}$, L-selectride ( $1 \mathrm{M}$ in THF, $2.29 \mathrm{~mL}, 2.29 \mathrm{mmol}, 1.5$ eq.) was added dropwise over $15 \mathrm{~min}$ and the resulting solution was stirred at this temperature for $45 \mathrm{~min}$. Lithium aluminum hydride ( $1 \mathrm{M}$ in THF, $3.82 \mathrm{~mL}, 3.82 \mathrm{mmol}, 2.5 \mathrm{eq}$.) was added, and the reaction mixture was warmed to $0{ }^{\circ} \mathrm{C}$. After stirring for $30 \mathrm{~min}$ at this temperature, EtOAc $(5 \mathrm{~mL})$ was added dropwise and a solution of $\mathrm{NaBO}_{3} \cdot 4 \mathrm{H}_{2} \mathrm{O}(1.18 \mathrm{~g}, 7.67 \mathrm{mmol}, 5.0$ eq.) in Rochelle's salt ( $1 / 2$ sat. aq., $30 \mathrm{~mL}$ ) was added carefully. The aqueous phase was extracted with EtOAc $(3 \times 25 \mathrm{~mL})$ and the combined organic phases were washed with brine (sat., $50 \mathrm{~mL}$ ), dried over $\mathrm{MgSO}_{4}$, and the solvent was removed under reduced pressure. The obtained hydroxy ketone $\mathbf{S 2 5}$ was used without further purification.

Analytically pure hydroxy ketone $\mathbf{S 2 5}$ was obtained by column chromatography (silica gel, nhexane/EtOAc $50: 1 \rightarrow 19: 1)$ as a colorless solid.

M.p.: $\quad 82-86^{\circ} \mathrm{C}(\mathrm{EtOAC})$.

TLC: $\quad R_{\mathrm{f}}=0.37$ (nhexane/EtOAc 9:1). 
1H-NMR: $\quad\left(700 \mathrm{MHz}, \mathrm{CDCl}_{3}\right) ; \delta[\mathrm{ppm}]=4.45(\mathrm{~d}, J=8.3 \mathrm{~Hz}, 1 \mathrm{H}), 4.23(\mathrm{t}, J=7.4 \mathrm{~Hz}, 1 \mathrm{H}), 3.17(\mathrm{~d}, J=$ $6.4 \mathrm{~Hz}, 1 \mathrm{H}), 2.61-2.54(\mathrm{~m}, 1 \mathrm{H}), 2.43(\mathrm{dd}, J=13.6,8.3 \mathrm{~Hz}, 1 \mathrm{H}), 2.33-2.26(\mathrm{~m}, 1 \mathrm{H}), 2.19$ $-2.12(\mathrm{~m}, 1 \mathrm{H}), 2.07-2.01(\mathrm{~m}, 1 \mathrm{H}), 1.70-1.64(\mathrm{~m}, 1 \mathrm{H}), 1.64-1.59(\mathrm{~m}, 2 \mathrm{H}), 1.58-1.51$ (m, 4H), $1.49-1.45(\mathrm{~m}, 1 \mathrm{H}), 1.45-1.41(\mathrm{~m}, 2 \mathrm{H}), 1.33(\mathrm{dd}, J=12.2,2.5 \mathrm{~Hz}, 1 \mathrm{H}), 1.09(\mathrm{~s}$, $3 \mathrm{H}), 1.06-1.01(\mathrm{~m}, 2 \mathrm{H}), 1.00(\mathrm{~s}, 3 \mathrm{H}), 0.98-0.97(\mathrm{~m}, 1 \mathrm{H}), 0.96(\mathrm{~d}, J=7.0 \mathrm{~Hz}, 3 \mathrm{H}), 0.94$ $-0.91(\mathrm{~m}, 1 \mathrm{H}), 0.85(\mathrm{~d}, J=6.9 \mathrm{~Hz}, 3 \mathrm{H}), 0.80-0.75(\mathrm{~m}, 6 \mathrm{H}), 0.77-0.70(\mathrm{~m}, 2 \mathrm{H}), 0.31(\mathrm{t}$, $J=4.3 \mathrm{~Hz}, 1 \mathrm{H})$.

13C-NMR: $\quad\left(176 \mathrm{MHz}, \mathrm{CDCl}_{3}\right) ; \delta[\mathrm{ppm}]=219.9,144.9,137.5,68.2,53.7,53.7,48.4,46.1,45.3,39.2$, $38.0,37.8,35.7,33.7,33.1,31.9,30.7,28.0,26.6,22.7,21.5,20.8,20.5,20.4,19.9,17.9$, 15.6, 7.8.

IR: $\quad\left(\right.$ neat); $\tilde{v}\left[\mathrm{~cm}^{-1}\right]=3443(\mathrm{br} \mathrm{w}), 2954(\mathrm{~s}), 2924(\mathrm{~s}), 2855(\mathrm{~m}), 1739(\mathrm{w}), 1685(\mathrm{w}), 1461(\mathrm{w})$, $1377(w), 1070(w)$.

HRMS: (ESI-TOF); $\mathrm{m} / \mathrm{z}$ calcd. for $\mathrm{C}_{28} \mathrm{H}_{44} \mathrm{O}_{2} \mathrm{Na}^{+}[\mathrm{M}+\mathrm{Na}]^{+}:$435.3234, found: 435.3249 .

Opt. act. $\quad[\mathrm{a}]_{\mathrm{D}}^{21}=+32.8\left(c=1.03, \mathrm{CHCl}_{3}\right)$.

13(14 $\rightarrow 8), 14(8 \rightarrow 7)$ Diabeo-3a,5-cyclo-5a,7ß(H)-ergosta-8-en-6a,14a-diol (S26)

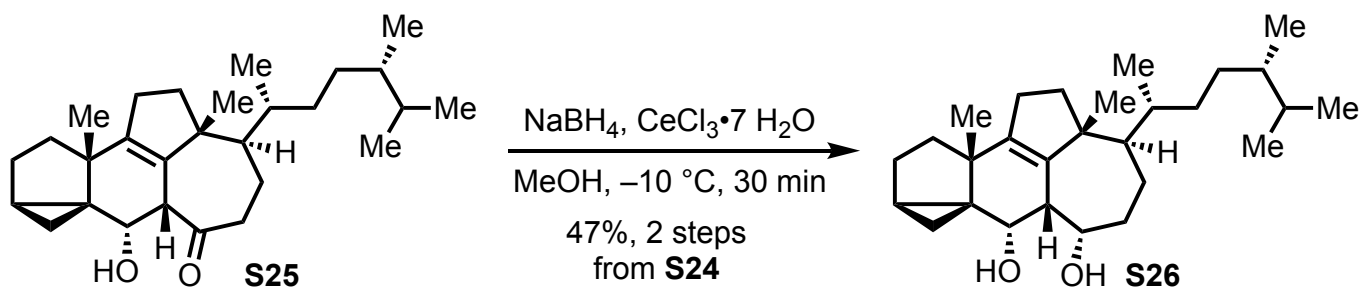

To a solution of crude hydroxy ketone $\mathbf{S} 25$ (631 mg, $1.53 \mathrm{mmol}, 1.0$ eq.) in $\mathrm{MeOH}(15 \mathrm{~mL})$ were added $\mathrm{CeCl}_{3} \cdot 7 \mathrm{H}_{2} \mathrm{O}$ (1.42 g, $3.82 \mathrm{mmol}, 2.5$ eq.) and $\mathrm{NaBH}_{4}\left(144 \mathrm{mg}, 3.82 \mathrm{mmol}, 2.5\right.$ eq.) at $-10{ }^{\circ} \mathrm{C}$. After stirring for $20 \mathrm{~min}$, the reaction mixture was diluted with EtOAc $(5 \mathrm{~mL})$, and $\mathrm{HCl}\left(1 \mathrm{M} \mathrm{in} \mathrm{H}_{2} \mathrm{O}, 10 \mathrm{~mL}\right)$ was added. The aqueous phase was extracted with EtOAc $(3 \times 20 \mathrm{~mL})$ and the combined organic phases were washed with brine (sat., $50 \mathrm{~mL}$ ), dried over $\mathrm{MgSO}_{4}$, and the solvent was removed under reduced pressure. Column chromatography (silica gel, nhexane/EtOAc 5:1) gave diol S26 (297 mg, $716 \mu \mathrm{mol}, 47 \%$ over two steps) as a colorless solid.

$\begin{array}{ll}\text { M.p.: } & 129-133^{\circ} \mathrm{C}(\mathrm{EtOAc}) . \\ \text { TLC: } & R_{\mathrm{f}}=0.22(\text { nhexane/EtOAc } 5: 1) . \\ \text { 1H-NMR: } & \left(700 \mathrm{MHz}, \mathrm{CDCl}_{3}\right) ; \delta[\mathrm{ppm}]=4.28(\mathrm{~d}, J=6.3 \mathrm{~Hz}, 1 \mathrm{H}), 4.23(\mathrm{~d}, J=5.8 \mathrm{~Hz}, 1 \mathrm{H}), 2.70(\mathrm{~d}, J \\ & =6.0 \mathrm{~Hz}, 1 \mathrm{H}), 2.29-2.23(\mathrm{~m}, 1 \mathrm{H}), 2.23-2.17(\mathrm{~m}, 1 \mathrm{H}), 1.93-1.87(\mathrm{~m}, 2 \mathrm{H}), 1.80-1.74 \\ & (\mathrm{~m}, 1 \mathrm{H}), 1.71-1.65(\mathrm{~m}, 1 \mathrm{H}), 1.65-1.60(\mathrm{~m}, 2 \mathrm{H}), 1.59-1.55(\mathrm{~m}, 3 \mathrm{H}), 1.51-1.49(\mathrm{~m},\end{array}$ 
$1 \mathrm{H}), 1.48-1.42(\mathrm{~m}, 4 \mathrm{H}), 1.42-1.37(\mathrm{~m}, 1 \mathrm{H}), 1.23-1.17(\mathrm{~m}, 2 \mathrm{H}), 1.11-1.06(\mathrm{~m}, 1 \mathrm{H})$, $1.05(\mathrm{~s}, 3 \mathrm{H}), 0.96(\mathrm{~s}, 3 \mathrm{H}), 0.95-0.92(\mathrm{~m}, 1 \mathrm{H}), 0.90(\mathrm{~d}, J=6.8 \mathrm{~Hz}, 3 \mathrm{H}), 0.86(\mathrm{~d}, J=6.9 \mathrm{~Hz}$, $3 \mathrm{H}), 0.79(\mathrm{~d}, J=6.8 \mathrm{~Hz}, 3 \mathrm{H}), 0.78(\mathrm{~d}, J=6.8 \mathrm{~Hz}, 3 \mathrm{H}), 0.69(\mathrm{dd}, J=8.4,5.0 \mathrm{~Hz}, 1 \mathrm{H}), 0.29$ (t, $J=4.6 \mathrm{~Hz}, 1 \mathrm{H})$.

13C-NMR: $\quad\left(151 \mathrm{MHz}, \mathrm{CDCl}_{3}\right) ; \delta[\mathrm{ppm}]=144.7,140.0,66.4,64.3,53.6,52.3,45.5,42.5,39.6,39.2$, $37.4,35.8,35.2,33.8,33.2,31.9,30.4,28.5,26.1,24.0,22.0,20.8,20.5,20.5,18.4,17.9$, 15.6, 7.1.

IR: $\quad\left(\right.$ neat); $\tilde{v}\left[\mathrm{~cm}^{-1}\right]=3405(\mathrm{br} \mathrm{w}), 2952(\mathrm{~m}), 2922(\mathrm{~s}), 2854(\mathrm{~m}), 1740(\mathrm{w}), 1456(\mathrm{~m}), 1377(\mathrm{w})$, $906(\mathrm{~m}), 734(\mathrm{~m})$.

HRMS: $\quad$ (ESI-TOF); $\mathrm{m} / z$ calcd. for $\mathrm{C}_{28} \mathrm{H}_{46} \mathrm{O}_{2} \mathrm{Na}^{+}[\mathrm{M}+\mathrm{Na}]+:$ 437.3390, found: 437.3407 .

Opt. act. $[\mathrm{a}]_{\mathrm{D}}^{22}=+73.3\left(c=1.00, \mathrm{CHCl}_{3}\right)$.

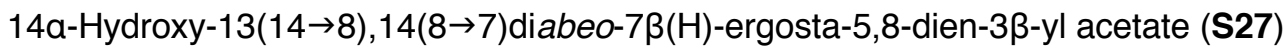
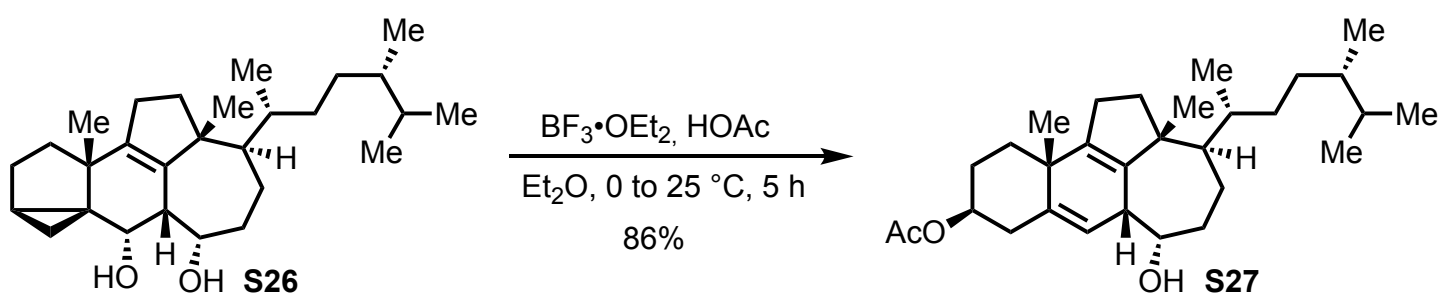

To a solution of diol S26 (276 mg, $666 \mu \mathrm{mol}, 1.0$ eq.) in $\mathrm{Et}_{2} \mathrm{O}(10 \mathrm{~mL})$ were added acetic acid (5 mL) and $\mathrm{BF}_{3} \cdot \mathrm{OEt}_{2}(5 \mathrm{~mL})$ at $0{ }^{\circ} \mathrm{C}$. The resulting solution was warmed to $25^{\circ} \mathrm{C}$ and stirred for $5 \mathrm{~h}$, before diluting with EtOAc $\left(10 \mathrm{~mL}\right.$ ). The reaction mixture was then carefully poured into $\mathrm{NaHCO}_{3}$ (sat. aq., $50 \mathrm{~mL}$ ) and the aqueous phase was extracted with EtOAc $(3 \times 20 \mathrm{~mL})$. The combined organic phases were washed sequentially with $\mathrm{NaHCO}_{3}$ (sat. aq., $20 \mathrm{~mL}$ ) and brine (sat., $20 \mathrm{~mL}$ ), dried over $\mathrm{MgSO}_{4}$, and the solvent was removed under reduced pressure. Column chromatography (silica gel, nhexane/EtOAc 5:1) gave acetate S27 (263 mg, $576 \mu \mathrm{mol}, 86 \%$ ) as a colorless oil.

TLC: $\quad R_{\mathrm{f}}=0.24$ (nhexane/EtOAc 5:1).

1H-NMR: $\quad\left(700 \mathrm{MHz}, \mathrm{CDCl}_{3}\right) ; \delta[\mathrm{ppm}]=5.38(\mathrm{dd}, J=3.2,1.6 \mathrm{~Hz}, 1 \mathrm{H}), 4.67-4.61(\mathrm{~m}, 1 \mathrm{H}), 3.83-$ $3.78(\mathrm{~m}, 1 \mathrm{H}), 3.00-2.97(\mathrm{~m}, 1 \mathrm{H}), 2.47(\mathrm{ddd}, J=12.5,5.2,1.9 \mathrm{~Hz}, 1 \mathrm{H}), 2.43-2.38(\mathrm{~m}$, $1 \mathrm{H}), 2.34-2.28(\mathrm{~m}, 1 \mathrm{H}), 2.26-2.21(\mathrm{~m}, 1 \mathrm{H}), 2.04(\mathrm{~s}, 3 \mathrm{H}), 1.98-1.89(\mathrm{~m}, 3 \mathrm{H}), 1.82$ (ddd, $J=12.0,7.6,2.6 \mathrm{~Hz}, 1 \mathrm{H}), 1.72-1.66(\mathrm{~m}, 2 \mathrm{H}), 1.63-1.59(\mathrm{~m}, 1 \mathrm{H}), 1.58-1.52(\mathrm{~m}, 3 \mathrm{H})$, $1.50-1.44(\mathrm{~m}, 3 \mathrm{H}), 1.41(\mathrm{dt}, J=12.9,4.5 \mathrm{~Hz}, 1 \mathrm{H}), 1.39-1.36(\mathrm{~m}, 1 \mathrm{H}), 1.30-1.24(\mathrm{~m}$, $2 \mathrm{H}), 1.22-1.18(\mathrm{~m}, 1 \mathrm{H}), 1.16(\mathrm{~s}, 3 \mathrm{H}), 0.96(\mathrm{~s}, 3 \mathrm{H}), 0.95-0.91(\mathrm{~m}, 1 \mathrm{H}), 0.90(\mathrm{~d}, J=6.9$ $\mathrm{Hz}, 3 \mathrm{H}), 0.85(\mathrm{~d}, J=6.9 \mathrm{~Hz}, 3 \mathrm{H}), 0.79(\mathrm{~d}, J=6.9 \mathrm{~Hz}, 3 \mathrm{H}), 0.77$ (d, $J=6.8 \mathrm{~Hz}, 3 \mathrm{H})$. 
13C-NMR: $\quad\left(176 \mathrm{MHz}, \mathrm{CDCl}_{3}\right) ; \delta[\mathrm{ppm}]=170.5,143.8,139.9,137.8,124.2,74.3,72.5,53.9,52.1$, $39.8,39.3,39.2,37.3,36.8,36.5,35.6,35.4,33.8,31.9,30.7,27.8,27.7,22.9,22.0,21.7$, 21.5, 20.5, 19.1, 17.9, 15.6 .

IR: $\quad\left(\right.$ neat); $\tilde{v}\left[\mathrm{~cm}^{-1}\right]=3394(\mathrm{br}), 2953(\mathrm{~s}), 2926(\mathrm{~s}), 2869(\mathrm{~m}), 1736(\mathrm{w}), 1464(\mathrm{w}), 1374(\mathrm{w})$, $1241(\mathrm{~m}), 1035(\mathrm{w})$.

HRMS: (ESI-TOF); $\mathrm{m} / z$ calcd. for $\mathrm{C}_{30} \mathrm{H}_{48} \mathrm{O}_{3} \mathrm{Na}^{+}[\mathrm{M}+\mathrm{Na}]^{+}:$479.3496, found: 479.3512 .

Opt. act. $[\mathrm{a}]_{\mathrm{D}}^{23}=+26.5\left(c=1.00, \mathrm{CHCl}_{3}\right)$.

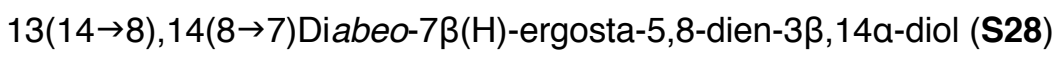
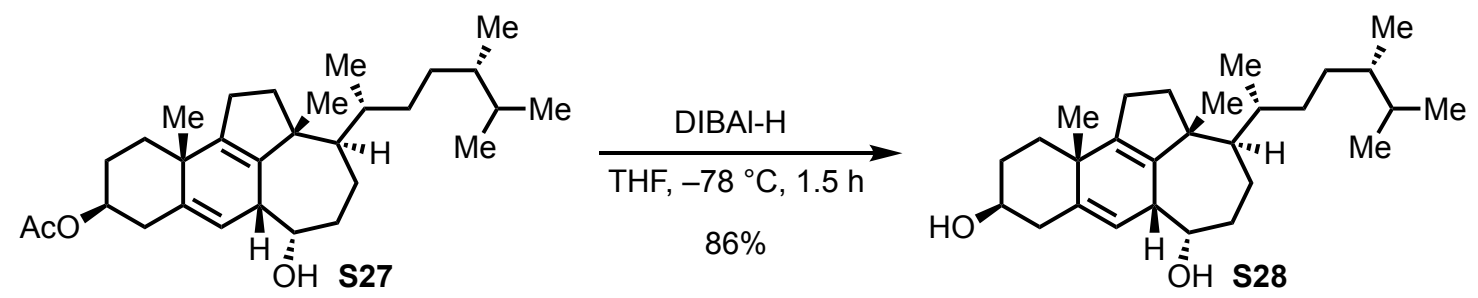

To a solution of acetate $\mathbf{S} 27$ (209 mg, $458 \mu \mathrm{mol}, 1.0 \mathrm{eq}$.) in THF $(5 \mathrm{~mL})$ at $-78{ }^{\circ} \mathrm{C}$ was added DIBAI-H $(1 \mathrm{M}$ in hexanes, $2.29 \mathrm{~mL}, 2.29 \mathrm{mmol}, 5.0$ eq.) and the resulting solution was stirred at this temperature for $1 \mathrm{~h}$. EtOAc $(1 \mathrm{~mL})$ and Rochelle's salt $(1 / 2$ sat. aq., $5 \mathrm{~mL})$ were added carefully and the mixture was vigorously stirred for $30 \mathrm{~min}$ while warming to $25^{\circ} \mathrm{C}$. The aqueous phase was extracted with EtOAc $(3 \times 5 \mathrm{~mL})$ and the combined organic phases were washed with brine (sat., $15 \mathrm{~mL}$ ), dried over $\mathrm{MgSO}_{4}$, and the solvent was removed under reduced pressure. Column chromatography (silica gel, nhexane/EtOAc 1:1) gave diol S28 (164 mg, $395 \mu \mathrm{mol}, 86 \%$ ) as a colorless solid.

M.p.: $\quad 120-122^{\circ} \mathrm{C}(\mathrm{EtOAC})$.

TLC: $\quad R_{\mathrm{f}}=0.24($ nhexane/EtOAc 1:1).

1H-NMR: $\quad\left(500 \mathrm{MHz}, \mathrm{CDCl}_{3}\right) ; \delta[\mathrm{ppm}]=5.40-5.28(\mathrm{~m}, 1 \mathrm{H}), 3.79(\mathrm{~d}, J=6.2 \mathrm{~Hz}, 1 \mathrm{H}), 3.61-3.52(\mathrm{~m}$, $1 \mathrm{H}), 3.03-2.95(\mathrm{~m}, 1 \mathrm{H}), 2.41(\mathrm{dd}, J=12.2,5.0 \mathrm{~Hz}, 1 \mathrm{H}), 2.37-2.29(\mathrm{~m}, 2 \mathrm{H}), 2.24(\mathrm{dd}, J$ $=15.5,8.4 \mathrm{~Hz}, 1 \mathrm{H}), 2.00-1.93(\mathrm{~m}, 1 \mathrm{H}), 1.93-1.86(\mathrm{~m}, 2 \mathrm{H}), 1.86-1.80(\mathrm{~m}, 2 \mathrm{H}), 1.74-$ $1.63(\mathrm{~m}, 2 \mathrm{H}), 1.62-1.51(\mathrm{~m}, 6 \mathrm{H}), 1.49-1.44(\mathrm{~m}, 2 \mathrm{H}), 1.41(\mathrm{dt}, J=12.7,4.1 \mathrm{~Hz}, 1 \mathrm{H}), 1.36$ (d, $J=10.5 \mathrm{~Hz}, 1 \mathrm{H}), 1.24-1.16(\mathrm{~m}, 2 \mathrm{H}), 1.14(\mathrm{~s}, 3 \mathrm{H}), 0.96(\mathrm{~s}, 3 \mathrm{H}), 0.95-0.91(\mathrm{~m}, 1 \mathrm{H})$, $0.89(\mathrm{~d}, J=6.9 \mathrm{~Hz}, 3 \mathrm{H}), 0.85(\mathrm{~d}, J=6.8 \mathrm{~Hz}, 3 \mathrm{H}), 0.80-0.76(\mathrm{~m}, 6 \mathrm{H})$.

13C-NMR: $\quad\left(126 \mathrm{MHz}, \mathrm{CDCl}_{3}\right) ; \delta[\mathrm{ppm}]=144.4,141.0,137.4,123.2,72.5,72.4,53.9,52.4,41.4,39.7$, $39.4,39.2,36.8,36.7,35.7,35.4,33.8,31.9,31.6,30.6,27.9,23.0,22.0,21.6,20.5,19.0$, 17.9, 15.6 . 
IR: $\quad\left(\right.$ neat); $\tilde{v}\left[\mathrm{~cm}^{-1}\right]=3391(\mathrm{br} \mathrm{w}), 2955(\mathrm{~s}), 2928(\mathrm{~s}), 2869(\mathrm{~m}), 1739(\mathrm{~m}), 1458(\mathrm{~m}), 1375(\mathrm{~m})$, $1288(\mathrm{w}), 1217(\mathrm{~m}), 1059(\mathrm{w})$.

HRMS: $\quad$ (ESI-TOF); $m / z$ calcd. for $\mathrm{C}_{28} \mathrm{H}_{46} \mathrm{O}_{2} \mathrm{Na}^{+}[\mathrm{M}+\mathrm{Na}]^{+}:$437.3390, found: 437.3386 .

Opt. act. $[\mathrm{a}]_{\mathrm{D}}^{22}=+40.8\left(c=1.00, \mathrm{CHCl}_{3}\right)$.

13(14 $\rightarrow 8), 14(8 \rightarrow 7)$ Diabeo-7ß(H)-ergosta-5,8-dien-3,14-dione (S29)
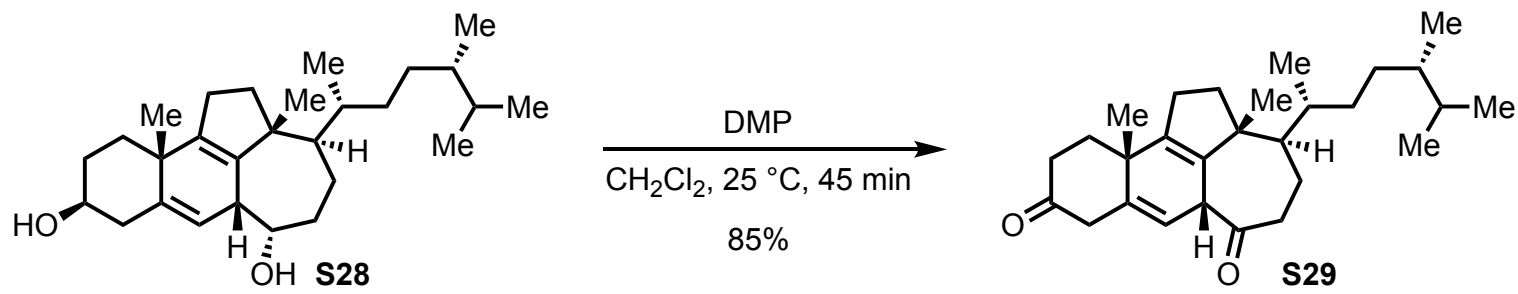

To a solution of diol S28 (164 mg, $395 \mu \mathrm{mol}, 1.0$ eq.) in $\mathrm{CH}_{2} \mathrm{Cl}_{2}(4 \mathrm{~mL})$ were added $\mathrm{NaHCO}_{3}(220 \mathrm{mg}$, $2.62 \mathrm{mmol}$, 6.6 eq.) and Dess-Martin periodinane (505 mg, $1.19 \mathrm{mmol}, 3.0$ eq.), and the resulting suspension was stirred at $25^{\circ} \mathrm{C}$. After $45 \mathrm{~min}, \mathrm{Na}_{2} \mathrm{~S}_{2} \mathrm{O}_{3}$ (sat. aq., $3 \mathrm{~mL}$ ) was added and after further stirring for $15 \mathrm{~min}$, the aqueous phase was extracted with $\mathrm{CH}_{2} \mathrm{Cl}_{2}(3 \times 10 \mathrm{~mL})$. The combined organic phases were washed sequentially with $\mathrm{NaHCO}_{3}$ (sat. aq., $15 \mathrm{~mL}$ ) and brine (sat., $15 \mathrm{~mL}$ ), dried over $\mathrm{MgSO}_{4}$, and the solvent was removed under reduced pressure. Column chromatography (silica gel, nhexane/EtOAc 5:1) gave dione $\mathbf{S 2 9}$ (138 mg, $336 \mu \mathrm{mol}, 85 \%)$ as a colorless solid.

M.p.: $\quad 103-105^{\circ} \mathrm{C}(\mathrm{EtOAc})$.

TLC: $\quad R_{\mathrm{f}}=0.19$ (nhexane/EtOAc 5:1).

1H-NMR: $\quad\left(700 \mathrm{MHz}, \mathrm{CDCl}_{3}\right) ; \delta[p p m]=5.23(\mathrm{t}, J=2.7 \mathrm{~Hz}, 1 \mathrm{H}), 3.57-3.53(\mathrm{~m}, 1 \mathrm{H}), 3.37(\mathrm{dt}, J=$ 16.2, 3.1 Hz, 1H), 2.98 (dd, $J=16.1,2.2 \mathrm{~Hz}, 1 \mathrm{H}$ ), 2.59 (ddd, $J=15.5,13.8,6.5 \mathrm{~Hz}, 1 \mathrm{H}$ ), $2.46-2.29(\mathrm{~m}, 5 \mathrm{H}), 2.20(\mathrm{dd}, J=11.3,7.9 \mathrm{~Hz}, 1 \mathrm{H}), 2.17-2.13(\mathrm{~m}, 1 \mathrm{H}), 2.03$ (ddd, $J=$ 13.2, 6.6, 2.4 Hz, 1H), $1.79-1.72(\mathrm{~m}, 1 \mathrm{H}), 1.62-1.52(\mathrm{~m}, 5 \mathrm{H}), 1.52-1.46(\mathrm{~m}, 2 \mathrm{H}), 1.44$ $-1.41(\mathrm{~m}, 2 \mathrm{H}), 1.40(\mathrm{~s}, 3 \mathrm{H}), 0.97-0.93(\mathrm{~m}, 6 \mathrm{H}), 0.93-0.88(\mathrm{~m}, 1 \mathrm{H}), 0.85(\mathrm{~d}, J=6.9 \mathrm{~Hz}$, $3 \mathrm{H}), 0.78(\mathrm{~d}, J=6.9 \mathrm{~Hz}, 3 \mathrm{H}), 0.76(\mathrm{~d}, J=6.8 \mathrm{~Hz}, 3 \mathrm{H})$.

13C-NMR: $\quad\left(176 \mathrm{MHz}, \mathrm{CDCl}_{3}\right) ; \delta[\mathrm{ppm}]=211.8,207.8,142.3,139.7,135.8,119.3,53.8,51.6,50.4$, 48.2, 41.2, 39.2, 38.2, 38.0, 37.1, 36.0, 35.8, 33.8, 31.9, 31.0, 27.7, 22.6, 22.1, 21.5, 20.5, 19.6, 17.9, 15.6.

IR: $\quad$ (neat); $\tilde{v}\left[\mathrm{~cm}^{-1}\right]=2955(\mathrm{~s}), 2925(\mathrm{~s}), 2854(\mathrm{~m}), 1716(\mathrm{~m}), 1458(\mathrm{w}), 1376(\mathrm{w})$.

HRMS: (ESI-TOF); $m / z$ calcd. for $\mathrm{C}_{28} \mathrm{H}_{42} \mathrm{O}_{2} \mathrm{Na}^{+}[\mathrm{M}+\mathrm{Na}]+:$ 433.3077, found: 433.3082 .

Opt. act. $[\mathrm{a}]_{\mathrm{D}}^{19}=-28.3\left(c=0.83, \mathrm{CHCl}_{3}\right)$. 

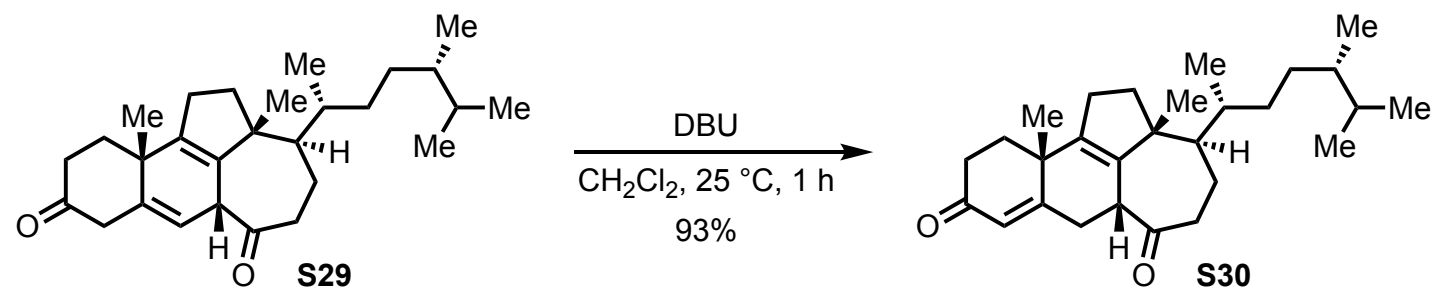

To a solution of dione $\mathbf{S 2 9}\left(138 \mathrm{mg}, 336 \mu \mathrm{mol}, 1.0\right.$ eq.) in $\mathrm{CH}_{2} \mathrm{Cl}_{2}$ (3.5 mL) was added 1,8diazabicyclo[5.4.0]undec-7-ene (10 mg, $66 \mu \mathrm{mol}, 0.2$ eq.) and the resulting solution was stirred at $25^{\circ} \mathrm{C}$ for $1 \mathrm{~h}$. The reaction mixture was then diluted with $\mathrm{CH}_{2} \mathrm{Cl}_{2}(2 \mathrm{~mL})$, and $\mathrm{NH}_{4} \mathrm{Cl}$ (sat. aq., $5 \mathrm{~mL}$ ) was added. The aqueous phase was extracted with $\mathrm{CH}_{2} \mathrm{Cl}_{2}(3 \times 10 \mathrm{~mL})$ and the combined organic phases were washed with brine (sat., $25 \mathrm{~mL}$ ), dried over $\mathrm{MgSO}_{4}$, and the solvent was removed under reduced pressure. Column chromatography (silica gel, nhexane/EtOAc 4:1 $\rightarrow$ 3:1) gave enone $\mathbf{S} 30$ (128 mg, $312 \mu \mathrm{mol}, 93 \%$ ) as a lightyellow oil.

TLC: $\quad R_{\mathrm{f}}=0.24($ nhexane/EtOAc $3: 1)$.

1H-NMR: $\quad\left(700 \mathrm{MHz}, \mathrm{CDCl}_{3}\right) ; \delta[\mathrm{ppm}]=5.86(\mathrm{~s}, 1 \mathrm{H}), 3.26-3.21(\mathrm{~m}, 1 \mathrm{H}), 2.87(\mathrm{~d}, J=12.8 \mathrm{~Hz}, 1 \mathrm{H})$, $2.57-2.48(\mathrm{~m}, 2 \mathrm{H}), 2.46$ (ddd, $J=15.8,6.8,1.9 \mathrm{~Hz}, 1 \mathrm{H}), 2.41(\mathrm{dd}, J=17.8,3.1 \mathrm{~Hz}, 1 \mathrm{H}$ ), $2.32-2.27(\mathrm{~m}, 2 \mathrm{H}), 2.21-2.13(\mathrm{~m}, 1 \mathrm{H}), 2.00-1.93(\mathrm{~m}, 2 \mathrm{H}), 1.76-1.70(\mathrm{~m}, 2 \mathrm{H}), 1.66-$ $1.60(\mathrm{~m}, 1 \mathrm{H}), 1.60-1.51(\mathrm{~m}, 3 \mathrm{H}), 1.51-1.40(\mathrm{~m}, 2 \mathrm{H}), 1.30(\mathrm{~s}, 3 \mathrm{H}), 1.27-1.17(\mathrm{~m}, 3 \mathrm{H})$, $1.08(\mathrm{~s}, 3 \mathrm{H}), 0.94(\mathrm{~d}, J=6.9 \mathrm{~Hz}, 3 \mathrm{H}), 0.85(\mathrm{~d}, J=6.8 \mathrm{~Hz}, 3 \mathrm{H}), 0.81-0.73(\mathrm{~m}, 7 \mathrm{H})$.

${ }^{13} \mathrm{C}-N M R: \quad\left(176 \mathrm{MHz}, \mathrm{CDCl}_{3}\right) ; \delta[\mathrm{ppm}]=211.4,198.6,166.9,143.4,137.7,126.1,55.1,53.6,46.1$, 45.0, 39.2, 38.0, 37.6, 35.4, 34.2, 33.7 (2C), 32.8, 31.9, 30.6, 27.2, 22.4, 21.6, 20.5, 20.4, 20.0, 17.9, 15.6 .

IR: $\quad\left(\right.$ neat); $\tilde{v}\left[\mathrm{~cm}^{-1}\right]=2955(\mathrm{~s}), 2927(\mathrm{~s}), 2866(\mathrm{~m}), 1739(\mathrm{~m}), 1709(\mathrm{~m}), 1672(\mathrm{~s}), 1456(\mathrm{~m})$, $1375(w), 1237(m), 1046(w)$.

HRMS: (ESI-TOF); $m / z$ calcd. for $\mathrm{C}_{28} \mathrm{H}_{42} \mathrm{O}_{2} \mathrm{Na}^{+}[\mathrm{M}+\mathrm{Na}]+:$ 433.3077, found: 433.3087.

Opt. act. $[\mathrm{a}]_{\mathrm{D}}^{24}=+106.7\left(c=1.00, \mathrm{CHCl}_{3}\right)$.
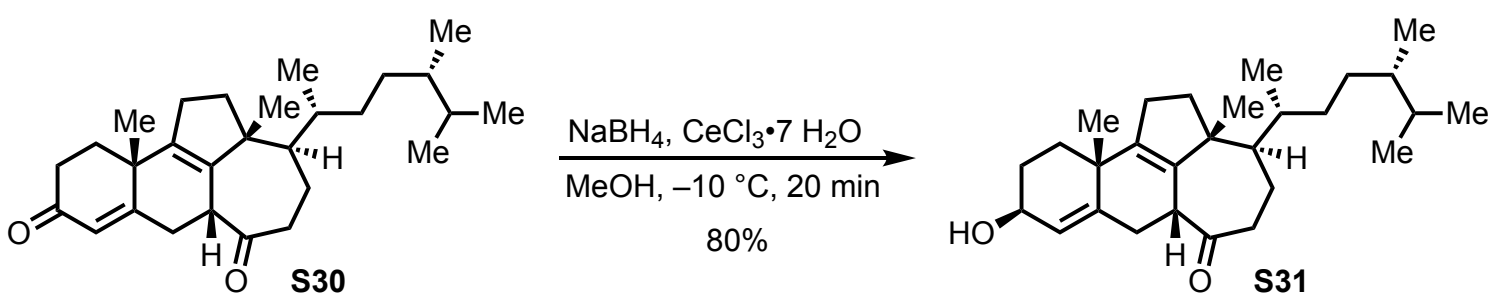
To a solution of enone $\mathbf{S} 30$ (18 mg, $44 \mu \mathrm{mol}, 1.0$ eq.) in $\mathrm{MeOH}(1.5 \mathrm{~mL})$ at $-10{ }^{\circ} \mathrm{C}$ were added $\mathrm{CeCl}_{3} \cdot 7 \mathrm{H}_{2} \mathrm{O}$ (41 mg, $110 \mu \mathrm{mol}, 2.5$ eq.) and $\mathrm{NaBH}_{4}(1.0 \mathrm{mg}, 26 \mu \mathrm{mol}, 0.6$ eq.) in three portions over $15 \mathrm{~min}$ at this temperature. The reaction mixture was diluted with EtOAc $(2 \mathrm{~mL})$, and $\mathrm{HCl}\left(1 \mathrm{M}\right.$ in $\left.\mathrm{H}_{2} \mathrm{O}, 5 \mathrm{~mL}\right)$ was added. The aqueous phase was extracted with EtOAc $(3 \times 5 \mathrm{~mL})$ and the combined organic phases were washed sequentially with $\mathrm{NaHCO}_{3}$ (sat. aq., $15 \mathrm{~mL}$ ) and brine (sat., $15 \mathrm{~mL}$ ), and dried over $\mathrm{MgSO}_{4}$. The solvent was removed under reduced pressure. Column chromatography (silica gel, nhexane/EtOAc 2:1) gave allylic alcohol S31 (14.5 mg, $35.1 \mu \mathrm{mol}, 80 \%)$ as a colorless oil.

TLC: $\quad R_{\mathrm{f}}=0.36($ nhexane/EtOAc 2:1).

'H-NMR: $\quad\left(700 \mathrm{MHz}, \mathrm{CDCl}_{3}\right) ; \delta[\mathrm{ppm}]=5.36(\mathrm{~s}, 1 \mathrm{H}), 4.16(\mathrm{t}, J=8.3 \mathrm{~Hz}, 1 \mathrm{H}), 2.98(\mathrm{~d}, J=6.7 \mathrm{~Hz}, 1 \mathrm{H})$, $2.63(\mathrm{~d}, J=12.7 \mathrm{~Hz}, 1 \mathrm{H}), 2.42-2.34(\mathrm{~m}, 2 \mathrm{H}), 2.29-2.16(\mathrm{~m}, 3 \mathrm{H}), 2.04-1.94(\mathrm{~m}, 2 \mathrm{H})$, 1.68 (dt, $J=13.3,3.5 \mathrm{~Hz}, 1 \mathrm{H}), 1.65-1.58(\mathrm{~m}, 3 \mathrm{H}), 1.58-1.53(\mathrm{~m}, 3 \mathrm{H}), 1.51-1.46(\mathrm{~m}$, $1 \mathrm{H}), 1.45-1.40(\mathrm{~m}, 2 \mathrm{H}), 1.39-1.32(\mathrm{~m}, 2 \mathrm{H}), 1.30-1.22(\mathrm{~m}, 3 \mathrm{H}), 1.20(\mathrm{~s}, 3 \mathrm{H}), 1.01(\mathrm{~s}$, $3 \mathrm{H}), 0.93(\mathrm{~d}, J=6.8 \mathrm{~Hz}, 3 \mathrm{H}), 0.85(\mathrm{~d}, J=6.8 \mathrm{~Hz}, 3 \mathrm{H}), 0.79(\mathrm{~d}, J=6.8 \mathrm{~Hz}, 3 \mathrm{H}), 0.77(\mathrm{~d}, J$ $=6.8 \mathrm{~Hz}, 3 \mathrm{H})$.

${ }^{13} \mathrm{C}-N M R: \quad\left(101 \mathrm{MHz}, \mathrm{CDCl}_{3}\right) ; \delta[\mathrm{ppm}]=214.2,145.2,142.8,137.4,125.7,67.8,54.2,53.5,46.6$, 45.4, 39.2, 37.9, 36.4, 35.5, 33.7, 33.1, 32.8, 31.9, 30.6, 28.8, 27.1, 23.9, 21.5, 20.5, 20.4, 20.3, 17.9, 15.6 .

IR: $\quad$ (neat); $\tilde{v}\left[\mathrm{~cm}^{-1}\right]=3406(\mathrm{br} \mathrm{w}), 2953(\mathrm{~s}), 2924(\mathrm{~s}), 2854(\mathrm{~m}), 1708(\mathrm{~m}), 1458(\mathrm{w}), 1376(\mathrm{w})$, $1065(w)$.

HRMS: $\quad$ (ESI-TOF); $m / z$ calcd. for $\mathrm{C}_{28} \mathrm{H}_{44} \mathrm{O}_{2} \mathrm{Na}^{+}[\mathrm{M}+\mathrm{Na}]+:$ 435.3234, found: 435.3255 .

Opt. act. $[\mathrm{a}]_{\mathrm{D}}^{23}=+34.9\left(c=1.00, \mathrm{CHCl}_{3}\right)$.

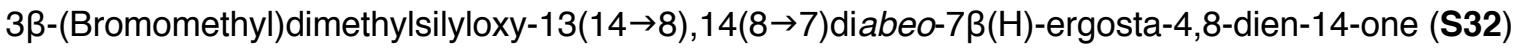
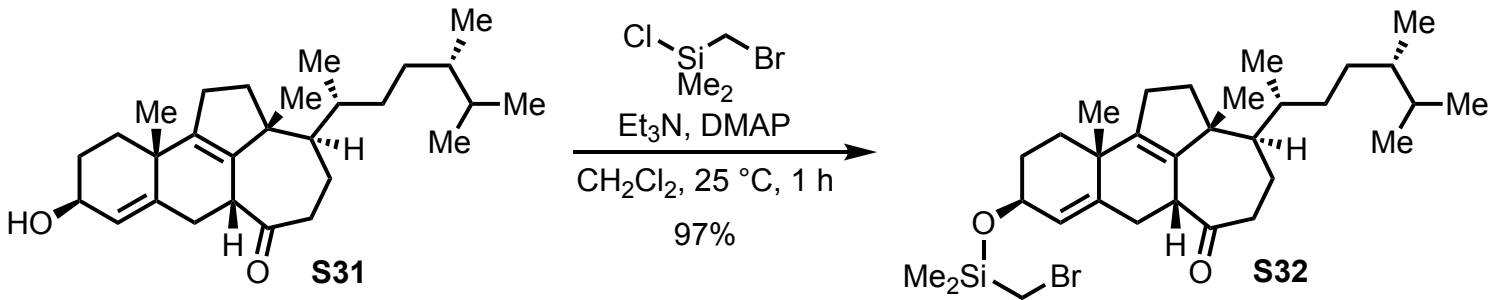

To a solution of allylic alcohol S31 $(4.5 \mathrm{mg}, 11 \mu \mathrm{mol}, 1.0$ eq. $)$ in $\mathrm{CH}_{2} \mathrm{Cl}_{2}(1 \mathrm{~mL})$ at $0{ }^{\circ} \mathrm{C}$ were added $\mathrm{Et}_{3} \mathrm{~N}$ (30 $\mu \mathrm{L}, 216 \mu \mathrm{mol}, 20$ eq.), 4-(dimethylamino)pyridine (0.3 mg, $2 \mu \mathrm{mol}, 0.2$ eq.) and (bromomethyl)chlorodimethylsilane ( $22 \mu \mathrm{L}, 165 \mu \mathrm{mol}, 15$ eq.) and the resulting solution was stirred at $25^{\circ} \mathrm{C}$ for $1 \mathrm{~h}$. The mixture was diluted with $\mathrm{CH}_{2} \mathrm{Cl}_{2}(2.5 \mathrm{~mL})$, and $\mathrm{NH}_{4} \mathrm{Cl}$ (sat. aq., $5 \mathrm{~mL}$ ) was added. The aqueous phase was 
extracted with $\mathrm{CH}_{2} \mathrm{Cl}_{2}(3 \times 5 \mathrm{~mL})$ and the combined organic phases were washed sequentially with $\mathrm{NH}_{4} \mathrm{Cl}$ (sat. aq., $4 \times 20 \mathrm{~mL}$ ), $\mathrm{NaHCO}_{3}$ (sat. aq., $15 \mathrm{~mL}$ ) and brine (sat., $15 \mathrm{~mL}$ ). The organic phase was dried over $\mathrm{MgSO}_{4}$, the solvent was removed under reduced pressure and column chromatography (silica gel, nhexane/EtOAc 19:1) gave bromide $\mathbf{S 3 2}(6.00 \mathrm{mg}, 10.6 \mu \mathrm{mol}, 97 \%)$ as a colorless oil.

TLC: $\quad R_{\mathrm{f}}=0.20($ nhexane/EtOAc 19:1).

1H-NMR: $\quad\left(600 \mathrm{MHz}, \mathrm{CDCl}_{3}\right) ; \delta[\mathrm{ppm}]=5.28(\mathrm{~s}, 1 \mathrm{H}), 4.27-4.21(\mathrm{~m}, 1 \mathrm{H}), 2.97(\mathrm{~d}, J=5.3 \mathrm{~Hz}, 1 \mathrm{H})$, $2.60(\mathrm{~d}, J=11.8 \mathrm{~Hz}, 1 \mathrm{H}), 2.47(\mathrm{~s}, 2 \mathrm{H}), 2.42-2.32(\mathrm{~m}, 2 \mathrm{H}), 2.27-2.16(\mathrm{~m}, 3 \mathrm{H}), 1.96$ (ddd, $J=12.3,7.5,3.5 \mathrm{~Hz}, 1 \mathrm{H}), 1.92-1.86(\mathrm{~m}, 1 \mathrm{H}), 1.72-1.63(\mathrm{~m}, 2 \mathrm{H}), 1.63-1.45(\mathrm{~m}, 6 \mathrm{H})$, $1.45-1.38(\mathrm{~m}, 2 \mathrm{H}), 1.37-1.30(\mathrm{~m}, 1 \mathrm{H}), 1.25-1.21(\mathrm{~m}, 1 \mathrm{H}), 1.18(\mathrm{~s}, 3 \mathrm{H}), 1.00(\mathrm{~s}, 3 \mathrm{H})$, $0.95-0.91(\mathrm{~m}, 4 \mathrm{H}), 0.84(\mathrm{~d}, J=6.8 \mathrm{~Hz}, 3 \mathrm{H}), 0.78(\mathrm{~d}, J=6.8 \mathrm{~Hz}, 3 \mathrm{H}), 0.76(\mathrm{~d}, J=6.8 \mathrm{~Hz}$, $3 \mathrm{H}), 0.75-0.69(\mathrm{~m}, 1 \mathrm{H}), 0.27(\mathrm{~s}, 6 \mathrm{H})$.

${ }^{13}$ C-NMR: $\quad\left(151 \mathrm{MHz}, \mathrm{CDCl}_{3}\right) ; \delta[\mathrm{ppm}]=214.3,145.2,142.6,137.3,125.6,69.2,54.2,53.4,46.6$, $45.4,39.2,37.9,36.3,35.4,33.7,33.1,32.8,31.9,30.6,29.0,27.1,23.8,21.5,20.5,20.4$, $20.3,17.9,16.6,15.6,-2.3,-2.4$.

IR: $\quad$ (neat); $\tilde{v}\left[\mathrm{~cm}^{-1}\right]=2955(\mathrm{~s}), 2926(\mathrm{~s}), 2856(\mathrm{~m}), 1708(\mathrm{~m}), 1460(\mathrm{w}), 1377(\mathrm{w}), 1254(\mathrm{~m})$, $1127(\mathrm{w}), 1073(\mathrm{~s}), 887(\mathrm{~m}), 839(\mathrm{~m}), 815(\mathrm{~m})$.

HRMS: $\quad$ (ESI-TOF); $\mathrm{m} / \mathrm{z}$ calcd. for $\mathrm{C}_{31} \mathrm{H}_{51} \mathrm{BrO}_{2} \mathrm{SiNa}^{+}[\mathrm{M}+\mathrm{Na}]^{+}: 585.2734$, found: 585.2756 .

Opt. act. $[\mathrm{a}]_{\mathrm{D}}^{23}=+12.3\left(c=1.00, \mathrm{CHCl}_{3}\right)$.

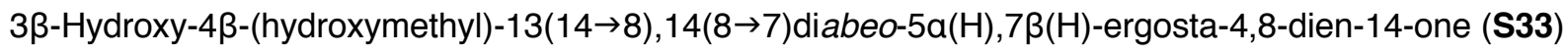
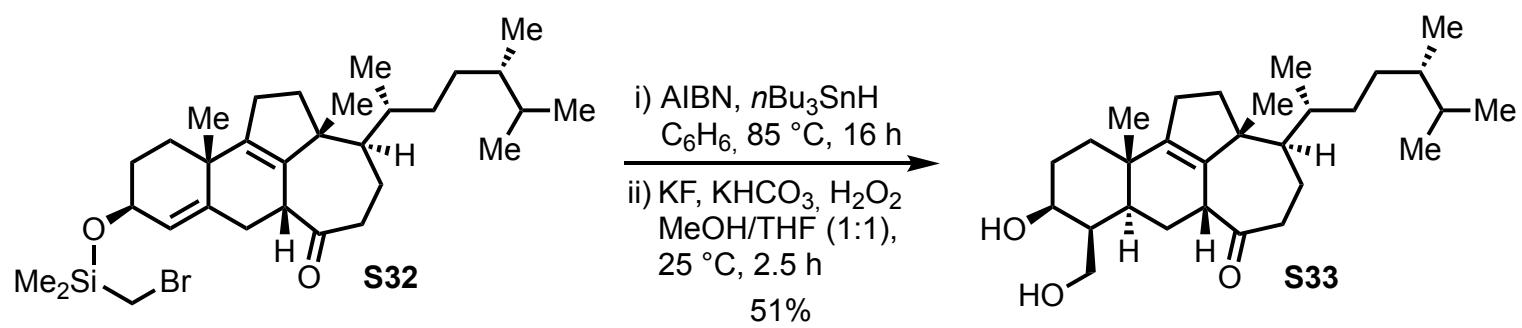

A solution of bromide S32 (15 mg, $27 \mu \mathrm{mol}, 1.0$ eq.), AIBN (4.4 mg, $27 \mu \mathrm{mol}, 1.0$ eq.) and $n \mathrm{Bu}_{3} \mathrm{SnH}$ (35.5 $\mu \mathrm{L}, 134 \mu \mathrm{mol}, 5.0$ eq.) in benzene $(1.5 \mathrm{~mL}$ ) was degassed applying three freeze-pump-thaw cycles. The reaction vessel was sealed, and it was heated to $85^{\circ} \mathrm{C}$ for $16 \mathrm{~h}$. After cooling to $25^{\circ} \mathrm{C}$, the solvent was removed under reduced pressure. The crude oxa-silolane was dissolved in $\mathrm{MeOH} / \mathrm{THF}(1: 1,1 \mathrm{~mL})$ and $\mathrm{KF}$ (15.6 mg, $270 \mu \mathrm{mol}, 10$ eq.), $\mathrm{KHCO}_{3}$ (26.8 mg, $270 \mu \mathrm{mol}, 10$ eq.), and $\mathrm{H}_{2} \mathrm{O}_{2}$ (35\% w/w in $\mathrm{H}_{2} \mathrm{O}, 0.5 \mathrm{~mL}$ ) were added. The mixture was stirred at $25^{\circ} \mathrm{C}$ for 30 min before $\mathrm{Na}_{2} \mathrm{~S}_{2} \mathrm{O}_{3}$ (sat. aq., $2 \mathrm{~mL}$ ) was carefully added. The aqueous phase was extracted with EtOAc $(3 \times 5 \mathrm{~mL})$ and the combined organic phases were washed with brine (sat., $15 \mathrm{~mL}$ ). The organic phase was dried over $\mathrm{MgSO}_{4}$, and the solvent was removed under 
reduced pressure. Column chromatography (silica gel, nhexane/EtOAc 2:1 $\rightarrow$ 1:1) gave diol S33 (6.2 mg, $14 \mu \mathrm{mol}, 51 \%)$ as a colorless oil in satisfactory purity for further transformation.

Analytically pure diol S33 was obtained using preparative HPLC (MACHEREY-NAGEL VP 250/16 NUCLEOSIL ${ }^{\circledR} 50-5$ Column; $n$-hexane/PrOH 9:1; 1 mL/min)

TLC: $\quad R_{\mathrm{f}}=0.21$ (nhexane/EtOAc 1:1).

1H-NMR: $\quad\left(700 \mathrm{MHz}, \mathrm{CDCl}_{3}\right) ; \delta[\mathrm{ppm}]=4.03(\mathrm{t}, J=10.4 \mathrm{~Hz}, 1 \mathrm{H}), 3.94(\mathrm{q}, J=7.3 \mathrm{~Hz}, 1 \mathrm{H}), 3.55(\mathrm{t}, J$ $=9.2 \mathrm{~Hz}, 1 \mathrm{H}), 3.14-3.09(\mathrm{~m}, 1 \mathrm{H}), 2.72-2.67(\mathrm{~m}, 1 \mathrm{H}), 2.54(\mathrm{ddd}, J=16.5,6.1,2.2 \mathrm{~Hz}$, $1 \mathrm{H}), 2.38-2.34(\mathrm{~m}, 1 \mathrm{H}), 2.33-2.30(\mathrm{~m}, 1 \mathrm{H}), 2.20-2.14(\mathrm{~m}, 2 \mathrm{H}), 2.00(\mathrm{~d}, J=11.3 \mathrm{~Hz}$, $1 \mathrm{H}), 1.78-1.69(\mathrm{~m}, 5 \mathrm{H}), 1.68-1.60(\mathrm{~m}, 5 \mathrm{H}), 1.53-1.49(\mathrm{~m}, 1 \mathrm{H}), 1.49-1.45(\mathrm{~m}, 1 \mathrm{H})$, 1.42 (tt, $J=12.6,4.3 \mathrm{~Hz}, 1 \mathrm{H}), 1.38-1.33(\mathrm{~m}, 1 \mathrm{H}), 1.18-1.12(\mathrm{~m}, 1 \mathrm{H}), 0.98(\mathrm{~s}, 3 \mathrm{H}), 0.97$ (s, 1H), $0.94-0.90(\mathrm{~m}, 4 \mathrm{H}), 0.85(\mathrm{~d}, J=6.8 \mathrm{~Hz}, 3 \mathrm{H}), 0.80-0.77(\mathrm{~m}, 9 \mathrm{H})$.

13C-NMR: $\quad\left(176 \mathrm{MHz}, \mathrm{CDCl}_{3}\right) ; \delta[\mathrm{ppm}]=213.4,147.1,136.1,74.5,60.3,55.8,53.8,48.2,45.9,45.5$, 41.6, 39.2, 38.7, 35.5, 35.2, 34.7, 33.7, 31.9, 30.7, 27.6, 26.9 (2C), 21.7, 20.5, 20.0, 19.9, 19.1, 17.9, 15.6.

IR: $\quad$ (neat); $\tilde{v}\left[\mathrm{~cm}^{-1}\right]=3390(\mathrm{br} \mathrm{w}), 2955$ (s), 2925 (s), $2855(\mathrm{~m}), 1704(\mathrm{w}), 1457(\mathrm{~m}), 1376(\mathrm{w})$, $1260(w), 798(w)$.

HRMS: (ESI-TOF); $\mathrm{m} / \mathrm{z}$ calcd. for $\mathrm{C}_{29} \mathrm{H}_{48} \mathrm{O}_{3} \mathrm{Na}^{+}[\mathrm{M}+\mathrm{Na}]+:$ 467.3496, found: 467.3473 .

Opt. act. $[\mathrm{a}]_{\mathrm{D}}^{20}=+19.4\left(c=0.35, \mathrm{CHCl}_{3}\right)$.

24-epi-Swinhoeisterol A (24-epi-1)
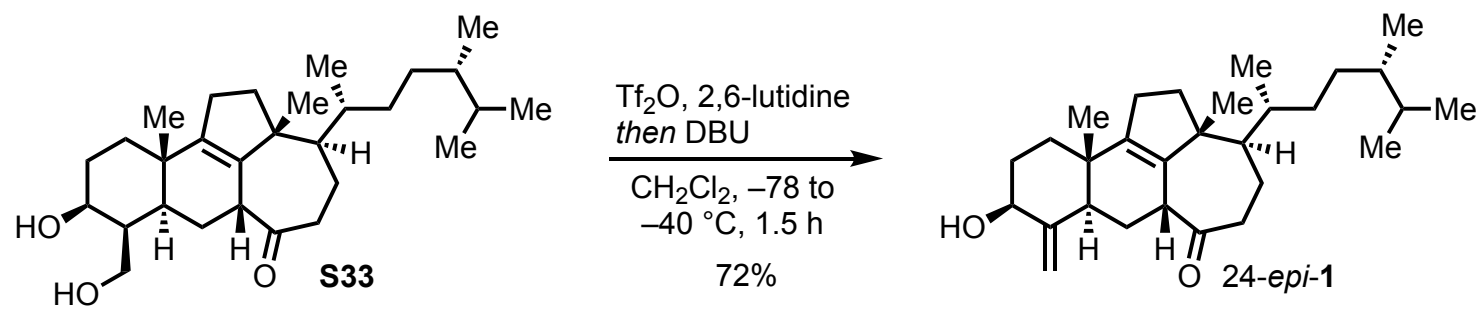

A solution of diol S33 (1.5 mg, $3.4 \mu \mathrm{mol}, 1.0$ eq.) and 2,6-lutidine ( $6 \mu \mathrm{L}, 51 \mu \mathrm{mol}, 15$ eq.) in $\mathrm{CH}_{2} \mathrm{Cl}_{2}(0.5 \mathrm{~mL})$ was cooled to $-78^{\circ} \mathrm{C}$. Triflic anhydride $\left(1 \mathrm{M}\right.$ in $\mathrm{CH}_{2} \mathrm{Cl}_{2}, 34 \mu \mathrm{L}, 34 \mu \mathrm{mol}, 10$ eq.) was added and the resulting mixture was stirred at this temperature for $10 \mathrm{~min}$ before $\mathrm{MeOH}(1.4 \mu \mathrm{L}, 34 \mu \mathrm{mol}, 10$ eq.) and 1,8diazabicyclo[5.4.0]undec-7-ene (10 $\mu \mathrm{L}, 68 \mu \mathrm{mol}, 20$ eq.) were added. The reaction mixture was allowed to warm to $-40{ }^{\circ} \mathrm{C}$ over $1.5 \mathrm{~h}$ before it was diluted with $\mathrm{CH}_{2} \mathrm{Cl}_{2}(1 \mathrm{~mL})$ and $\mathrm{HCl}\left(1 \mathrm{M}\right.$ in $\left.\mathrm{H}_{2} \mathrm{O}, 1 \mathrm{~mL}\right)$ was added. The aqueous phase was extracted with $\mathrm{CH}_{2} \mathrm{Cl}_{2}(3 \times 5 \mathrm{~mL})$ and the combined organic phases were washed sequentially with $\mathrm{NaHCO}_{3}$ (sat. aq., $15 \mathrm{~mL}$ ) and brine (sat., $15 \mathrm{~mL}$ ), dried over $\mathrm{MgSO}_{4}$, and the solvent was 
removed under reduced pressure. Column chromatography (silica gel, nhexane/EtOAc 3:1) gave 24-episwinhoeisterol A (24-epi-1) (1.0 mg, $2.4 \mu \mathrm{mol}, 72 \%)$ as a colorless oil.

TLC: $\quad R_{\mathrm{f}}=0.29($ nhexane/EtOAc $3: 1)$.

1H-NMR: $\quad\left(600 \mathrm{MHz}, \mathrm{CDCl}_{3}\right) ; \delta[\mathrm{ppm}]=5.09(\mathrm{~s}, 1 \mathrm{H}), 4.69(\mathrm{~s}, 1 \mathrm{H}), 4.04(\mathrm{dd}, J=11.9,6.2 \mathrm{~Hz}, 1 \mathrm{H})$, $3.16-3.12(\mathrm{~m}, 1 \mathrm{H}), 2.53(\mathrm{ddd}, J=16.3,6.2,2.2 \mathrm{~Hz}, 1 \mathrm{H}), 2.39-2.33(\mathrm{~m}, 2 \mathrm{H}), 2.31-2.26$ $(\mathrm{m}, 1 \mathrm{H}), 2.21$ (ddd, $J=9.0,6.3,2.7 \mathrm{~Hz}, 1 \mathrm{H}), 2.14(\mathrm{~d}, J=13.2 \mathrm{~Hz}, 1 \mathrm{H}), 2.06-2.02(\mathrm{~m}, 1 \mathrm{H})$, $2.00(\mathrm{~d}, J=13.9 \mathrm{~Hz}, 1 \mathrm{H}), 1.80-1.74(\mathrm{~m}, 3 \mathrm{H}), 1.71-1.69(\mathrm{~m}, 1 \mathrm{H}), 1.67-1.63(\mathrm{~m}, 2 \mathrm{H})$, $1.62-1.60(\mathrm{~m}, 1 \mathrm{H}), 1.60-1.58(\mathrm{~m}, 1 \mathrm{H}), 1.48-1.46(\mathrm{~m}, 1 \mathrm{H}), 1.38-1.36(\mathrm{~m}, 1 \mathrm{H}), 1.36-$ $1.34(\mathrm{~m}, 1 \mathrm{H}), 1.34-1.32(\mathrm{~m}, 1 \mathrm{H}), 1.22-1.18(\mathrm{~m}, 3 \mathrm{H}), 1.01(\mathrm{~s}, 3 \mathrm{H}), 0.94-0.92(\mathrm{~m}, 4 \mathrm{H})$, $0.85(\mathrm{~d}, J=6.8 \mathrm{~Hz}, 3 \mathrm{H}), 0.80-0.77(\mathrm{~m}, 9 \mathrm{H})$.

13C-NMR: $\quad\left(176 \mathrm{MHz}, \mathrm{CDCl}_{3}\right) ; \delta[\mathrm{ppm}]=213.6,151.9,146.9,135.9,102.9,73.1,55.9,53.8,45.4$, $45.3,44.4,39.2,38.7,37.7,35.5,34.8,33.7,32.5,31.9,30.7,27.8,24.5,21.7,20.5,20.1$, 20.0, 17.9, 17.9, 15.6 .

IR: $\quad\left(\right.$ neat); $\tilde{v}\left[\mathrm{~cm}^{-1}\right]=2952(\mathrm{~m}), 2924(\mathrm{~s}), 2853(\mathrm{~m}), 1739(\mathrm{w}), 1707(\mathrm{w}), 1459(\mathrm{w}), 1376(\mathrm{w})$.

HRMS: (ESI-TOF); $\mathrm{m} / \mathrm{z}$ calcd. for $\mathrm{C}_{29} \mathrm{H}_{46} \mathrm{O}_{2} \mathrm{Na}^{+}[\mathrm{M}+\mathrm{Na}]^{+}:$449.3390, found: 449.3410 .

Opt. act. $\quad[a]_{D}^{23}=+44.1\left(c=0.1, \mathrm{CHCl}_{3}\right)$. 


\section{NMR Comparisons}

Table S1 NMR comparison of natural dankasterone B (2) ${ }^{[3]}$ and synthetic material.

\begin{tabular}{|c|c|c|c|c|}
\hline \multirow{2}{*}{ \# } & \multicolumn{2}{|c|}{${ }^{1} \mathrm{H}-\mathrm{NMR}$} & \multicolumn{2}{|c|}{${ }^{13} \mathrm{C}-\mathrm{NMR}$} \\
\hline & natural[3],a & synthetic $^{b}$ & natural[3],c & synthetic $^{d}$ \\
\hline 1 & $\begin{array}{c}1.54 \mathrm{td}(13.2,5.7) ; 1.31 \mathrm{ddt} \\
(13.2,6.9,2.5)\end{array}$ & $\begin{array}{c}1.54 \mathrm{td}(12.9,5.7) ; 1.31 \mathrm{ddt}(13.3 \\
6.8,2.4)\end{array}$ & 32.7 & 32.7 \\
\hline 2 & $\begin{array}{c}2.21 \mathrm{ddt}(13.0,5.7,2.2) ; 2.29 \mathrm{td} \\
(13.0,6.9)\end{array}$ & $2.22 \mathrm{dt}(5.7,2.1) ; 2.29 \mathrm{~m}$ & 36.8 & 36.8 \\
\hline 3 & - & - & 207.6 & 207.6 \\
\hline 4 & 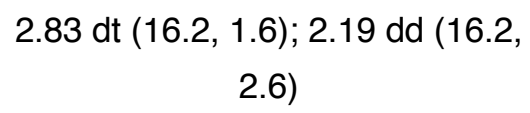 & $2.84 \mathrm{dt}(16.5,2.0) ; 2.20 \mathrm{~d}(6.1)$ & 35.8 & 35.8 \\
\hline 5 & $2.89 \mathrm{~m}$ & $2.89 \mathrm{~m}$ & 50.0 & 50.0 \\
\hline 6 & - & - & 208.5 & 208.5 \\
\hline 7 & $2.95 \mathrm{dd}(13.2,1.8) ; 1.95 \mathrm{~d}(13.2)$ & $2.95 \mathrm{dd}(13.2,1.8) ; 1.95 \mathrm{~d}(13.5)$ & 40.0 & 40.0 \\
\hline 8 & - & - & 65.6 & 65.6 \\
\hline 9 & $3.05 \mathrm{td}(9.6,1.8)$ & $3.05 \mathrm{t}(9.9)$ & 53.2 & 53.2 \\
\hline 10 & - & - & 40.5 & 40.5 \\
\hline 11 & $2.31 \mathrm{~m} ; 2.10 \mathrm{~m}$ & $2.31 \mathrm{~m} ; 2.10 \mathrm{~m}$ & 25.5 & 25.5 \\
\hline 12 & $2.14 \mathrm{~m} ; 1.65 \mathrm{~m}$ & $2.14 \mathrm{~m} ; 1.65 \mathrm{~m}$ & 34.1 & 34.1 \\
\hline 13 & - & - & 60.1 & 60.0 \\
\hline 14 & - & - & 214.7 & 214.7 \\
\hline 15 & $\begin{array}{c}2.78 \text { ddd }(13.0,12.8,5.9) ; 2.36 \\
\text { ddd }(13.0,4.3,2.5)\end{array}$ & $\begin{array}{c}2.79 \text { ddd }(14.3,12.8,5.9) ; 2.36 \text { ddd } \\
(12.7,4.2,2.5)\end{array}$ & 38.5 & 38.5 \\
\hline 16 & $1.99 \mathrm{~m} ; 1.63 \mathrm{~m}$ & $1.99 \mathrm{~m} ; 1.64 \mathrm{~m}$ & 27.2 & 27.2 \\
\hline 17 & $1.95 \mathrm{~m}$ & $1.95 \mathrm{~m}$ & 45.4 & 45.4 \\
\hline 18 & $0.75 \mathrm{~s}$ & $0.75 \mathrm{~s}$ & 15.2 & 15.1 \\
\hline 19 & $1.27 \mathrm{~s}$ & $1.27 \mathrm{~s}$ & 23.4 & 23.4 \\
\hline 20 & $2.42 \mathrm{~m}$ & $2.42 p(6.9)$ & 36.9 & 36.9 \\
\hline 21 & $1.14 \mathrm{~d}(6.9)$ & $1.15 \mathrm{~d}(7.1)$ & 24.1 & 24.1 \\
\hline 22 & $5.22 \mathrm{dd}(15.3,6.9)$ & $5.23 \mathrm{~m}$ & 132.0 & 132.0 \\
\hline 23 & $5.25 \mathrm{dd}(15.3,6.8)$ & $5.23 \mathrm{~m}$ & 135.2 & 135.2 \\
\hline 24 & $1.84 \mathrm{~m}$ & $1.84 \mathrm{~m}$ & 43.2 & 43.2 \\
\hline 241 & $0.88 d(6.8)$ & $0.88 d(6.8)$ & 17.5 & 17.5 \\
\hline 25 & $1.45 \mathrm{~m}$ & $1.45 \mathrm{~m}$ & 33.0 & 33.0 \\
\hline 26 & $0.79 d(6.8)$ & $0.79 \mathrm{~d}(6.8)$ & 19.7 & 19.7 \\
\hline 27 & $0.81 d(6.8)$ & $0.81 d(6.7)$ & 20.0 & 20.0 \\
\hline
\end{tabular}

All chemical shifts are reported in ppm. Coupling constants are in parentheses and are reported in $\mathrm{Hz}$. $\mathrm{m}=$ centered multiplet. All spectra were measured in $\mathrm{CDCl}_{3}$ and are referenced to the residual solvent peak at $\delta_{\mathrm{H}}=7.26 \mathrm{ppm}$ and $\delta_{\mathrm{C}}=77.00 \mathrm{ppm}$. ${ }^{a}$ Recorded at $500 \mathrm{MHz}$. b recorded at $700 \mathrm{MHz}$. ${ }^{\mathrm{c}}$ recorded at $126 \mathrm{MHz}$. d recorded at $176 \mathrm{MHz}$. 
Table S2 NMR comparison of natural dankasterone $A\left(\Delta^{4-2}\right)^{[4]}$ and synthetic material.

\begin{tabular}{|c|c|c|c|c|}
\hline \multirow{2}{*}{ \# } & \multicolumn{2}{|c|}{${ }^{1} \mathrm{H}-\mathrm{NMR}$} & \multicolumn{2}{|c|}{${ }^{13} \mathrm{C}-\mathrm{NMR}$} \\
\hline & natural[4],a & synthetic $^{b}$ & natural $[4], c$ & synthetic $^{d}$ \\
\hline 1 & $2.08 \mathrm{dd}(13.3,5.1) ; 2.04 \mathrm{~m}$ & $2.08 \mathrm{~m} ; 2.03 \mathrm{~m}$ & 38.9 & 38.9 \\
\hline 2 & $2.53 \mathrm{dt}(17.6,6.2) ; 2.46 \mathrm{~m}$ & $2.53 \mathrm{~m} ; 2.46 \mathrm{~m}$ & 34.3 & 34.4 \\
\hline 3 & - & - & 199.1 & 199.1 \\
\hline 4 & $6.36 \mathrm{~s}$ & $6.36 \mathrm{~s}$ & 126.5 & 126.5 \\
\hline 5 & - & - & 156.1 & 156.0 \\
\hline 6 & - & - & 200.0 & 200.0 \\
\hline 7 & $2.66 \mathrm{dd}(16.8,1.3) ; 2.50 \mathrm{~d}(16.8)$ & $2.65 \mathrm{dd}(16.8,1.6) ; 2.50 \mathrm{~m}$ & 40.8 & 40.9 \\
\hline 8 & - & - & 62.2 & 62.2 \\
\hline 9 & $2.81 \mathrm{td}(9.0,1.3)$ & $2.81 \mathrm{~m}$ & 49.4 & 49.4 \\
\hline 10 & - & - & 36.0 & 36.0 \\
\hline 11 & $2.02 \mathrm{~m} ; 1.85 \mathrm{~m}$ & $2.00 \mathrm{~m} ; 1.85 \mathrm{~m}$ & 25.1 & 25.1 \\
\hline 12 & $1.77 \mathrm{dt}(13.0,7.2) ; 1.71 \mathrm{~m}$ & $1.77 \mathrm{dt}(13.0,7.4) ; 1.71 \mathrm{~m}$ & $38.3^{e}$ & 38.3 \\
\hline 13 & - & - & $54.0 \mathrm{e}$ & 54.0 \\
\hline 14 & - & - & 214.8 & 214.7 \\
\hline 15 & $2.48 \mathrm{~m}(2 \mathrm{H})$ & $2.48 \mathrm{~m}(2 \mathrm{H})$ & 37.9 & 37.9 \\
\hline 16 & $1.90 ; 1.69 \mathrm{~m}$ & $1.90 \mathrm{~m} ; 1.69 \mathrm{~m}$ & 23.2 & 23.2 \\
\hline 17 & $1.48 \mathrm{dd}(13.2,4.2)$ & $1.48 \mathrm{~m}$ & 49.3 & 49.4 \\
\hline 18 & $0.98 \mathrm{~s}$ & $0.98 \mathrm{~s}$ & 17.1 & 17.1 \\
\hline 19 & $1.26 \mathrm{~s}$ & $1.26 \mathrm{~s}$ & 24.0 & 24.0 \\
\hline 20 & $2.42 \mathrm{~m}$ & $2.41 \mathrm{td}(7.4,2.6)$ & 37.2 & 37.2 \\
\hline 21 & $1.09 \mathrm{~d}(6.8)$ & $1.09 \mathrm{~d}(7.0)$ & 23.6 & 23.6 \\
\hline 22 & $5.25 \mathrm{dd}(15.1,5.0)$ & $5.27 \mathrm{~m}$ & 132.3 & 132.3 \\
\hline 23 & 5.29 dd $(15.1,3.5)$ & $5.27 \mathrm{~m}$ & 135.1 & 135.1 \\
\hline 24 & $1.88 \mathrm{~m}$ & $1.88 \mathrm{~m}$ & 43.2 & 43.2 \\
\hline 241 & $0.91 \mathrm{~d}(6.8)$ & $0.91 \mathrm{~d}(6.9)$ & 17.6 & 17.6 \\
\hline 25 & 1.47 octet $(6.8)$ & $1.48 \mathrm{~m}$ & 33.0 & 33.1 \\
\hline 26 & $0.84 \mathrm{~d}(6.8)$ & $0.83 \mathrm{~d}(6.8)$ & 20.0 & 20.0 \\
\hline 27 & $0.81 \mathrm{~d}(6.8)$ & $0.81 \mathrm{~d}(6.8)$ & 19.7 & 19.7 \\
\hline
\end{tabular}

All chemical shifts are reported in ppm. Coupling constants are in parentheses and are reported in $\mathrm{Hz}$. $\mathrm{m}=$ centered multiplet. All spectra were measured in $\mathrm{CDCl}_{3}$ and are referenced to the residual solvent peak at $\delta_{H}=7.26 \mathrm{ppm}$ and $\delta_{\mathrm{C}}=77.00 \mathrm{ppm}$. a Recorded at $500 \mathrm{MHz}$. ${ }^{b}$ recorded at $700 \mathrm{MHz}$. ${ }^{c}$ recorded at $126 \mathrm{MHz}$. d recorded at $176 \mathrm{MHz}$. e originally both assigned to $\mathrm{C} 12$. 
Table S3 NMR comparison of natural periconiastone $(3)^{[5]}$ and synthetic material.

\begin{tabular}{|c|c|c|c|c|}
\hline \multirow{2}{*}{ \# } & \multicolumn{2}{|c|}{${ }^{1} \mathrm{H}-\mathrm{NMR}$} & \multicolumn{2}{|c|}{${ }^{13} \mathrm{C}-\mathrm{NMR}$} \\
\hline & natural[5],a & synthetic $^{b}$ & natural[5],c & synthetic $^{d}$ \\
\hline 1 & $1.53 \mathrm{~m} ; 1.81 \mathrm{~m}$ & $1.51 \mathrm{~m}, 1.84 \mathrm{~m}$ & 38.5 & 38.5 \\
\hline 2 & $2.46 \mathrm{~m}, 2.87 \mathrm{~m}$ & $2.46 \mathrm{~m}, 2.87 \mathrm{ddd}(19.9,11.8,9.0)$ & 36.8 & 36.8 \\
\hline 3 & - & - & 209.2 & 209.2 \\
\hline 4 & $3.12 \mathrm{~d}(4.0)$ & $3.12 \mathrm{~d}(3.9)$ & 65.3 & 65.3 \\
\hline 5 & $2.45 \mathrm{~d}(4.0)$ & $2.46 \mathrm{~m}$ & 58.8 & 58.8 \\
\hline 6 & - & - & 213.2 & 213.1 \\
\hline 7 & $\begin{array}{c}2.12 \mathrm{~d}(18.5) ; 3.21 \mathrm{dd}(18.5, \\
2.4)\end{array}$ & $2.13 \mathrm{~m}, 3.21 \mathrm{~d}(18.5)$ & 37.4 & 37.4 \\
\hline 8 & - & - & 56.0 & 56.0 \\
\hline 9 & $2.80 \mathrm{~m}$ & $2.80 \mathrm{t}(10.0)$ & 42.3 & 42.3 \\
\hline 10 & - & - & 33.7 & 33.6 \\
\hline 11 & $1.85 \mathrm{~m}, 1.88 \mathrm{~m}$ & $1.84 \mathrm{~m}(2 \mathrm{H})$ & 22.7 & 22.7 \\
\hline 12 & $1.58 \mathrm{~m}, 2.04 \mathrm{~m}$ & $1.51 \mathrm{~m}, 2.03 \mathrm{ddd}(14.2,9.7,5.3)$ & 37.6 & 37.6 \\
\hline 13 & - & - & 47.9 & 47.8 \\
\hline 14 & - & - & 73.1 & 73.1 \\
\hline 15 & $1.88 \mathrm{~m}, 2.13 \mathrm{~m}$ & $1.84 \mathrm{~m}, 2.13 \mathrm{~m}$ & 34.3 & 34.3 \\
\hline 16 & $1.48 \mathrm{~m}, 1.92 \mathrm{~m}$ & $1.51 \mathrm{~m}, 1.94 \mathrm{~m}$ & 20.6 & 20.6 \\
\hline 17 & $1.55 \mathrm{~m}$ & $1.51 \mathrm{~m}$ & 48.2 & 48.2 \\
\hline 18 & $1.22 \mathrm{~s}$ & $1.22 \mathrm{~s}$ & 19.3 & 19.3 \\
\hline 19 & $0.99 \mathrm{~s}$ & $0.99 \mathrm{~s}$ & 26.6 & 26.5 \\
\hline 20 & $2.49 \mathrm{~m}$ & $2.47 \mathrm{~m}$ & 37.4 & 37.4 \\
\hline 21 & $1.13 \mathrm{~d}(7.1)$ & $1.13 \mathrm{~d}(6.9)$ & 24.5 & 24.4 \\
\hline 22 & $5.52 \mathrm{dd}(15.3,7.9)$ & $5.51 \mathrm{dd}(15.4,8.1)$ & 134.6 & 134.6 \\
\hline 23 & $5.30 \mathrm{dd}(15.3,8.2)$ & $5.30 \mathrm{dd}(15.4,8.2)$ & 134.5 & 134.5 \\
\hline 24 & $1.86 \mathrm{~m}$ & $1.84 \mathrm{~m}$ & 44.0 & 44.0 \\
\hline 241 & $0.92 d(6.8)$ & $0.92 d(6.8)$ & 33.9 & 33.9 \\
\hline 25 & $1.45 \mathrm{~m}$ & $1.51 \mathrm{~m}$ & 20.7 & 20.7 \\
\hline 26 & $0.86 d(6.7)$ & $0.85(6.8)$ & 20.4 & 20.3 \\
\hline 27 & $0.84 d(6.7)$ & $0.84(7.3)$ & 18.4 & 18.3 \\
\hline $14-\mathrm{OH}$ & $6.63 \mathrm{~s}$ & $6.64 \mathrm{~s}$ & - & - \\
\hline
\end{tabular}

All chemical shifts are reported in ppm. Coupling constants are in parentheses and are reported in $\mathrm{Hz} . \mathrm{m}=$ centered multiplet. All spectra were measured in pyridine- $d_{5}$ and are referenced to the residual solvent peak at $\delta_{\mathrm{H}}=8.74 \mathrm{ppm}$ and $\delta_{\mathrm{C}}=150.35 \mathrm{ppm}$. ${ }^{a}$ Recorded at $400 \mathrm{MHz}$. ${ }^{b}$ recorded at $600 \mathrm{MHz}$. ${ }^{\mathrm{c}}$ recorded at $100 \mathrm{MHz}$. d recorded at $151 \mathrm{MHz}$. 
Table S4. ${ }^{1} \mathrm{H}-\mathrm{NMR}$ comparison of synthetic swinhoeisterol A, natural swinhoeisterol A (1) ${ }^{[6]}$, and synthetic 24-epi-swinhoeisterol A (24-epi-1).

\begin{tabular}{|c|c|c|c|}
\hline$\#$ & Synthetica & Natural[6],b & Synthetic 24-epia \\
\hline 1 & $1.71 \mathrm{~m}, 1.36 \mathrm{td}(13.3,4.2)$ & $1.70 \mathrm{ov} ; 1.35 \mathrm{~m}$ & $1.70 \mathrm{~m} ; 1.35 \mathrm{~m}$ \\
\hline 2 & $2.04 \mathrm{~m}, 1.48 \mathrm{qd}(12.7,4.3)$ & $2.05 \mathrm{~m} ; 1.46 \mathrm{ov}$ & $2.05 \mathrm{~m} ; 1.46 \mathrm{~m}$ \\
\hline 3 & $4.04 \mathrm{dd}(11.9,5.8)$ & $4.03 \mathrm{dd}(14.5,5.8)$ & $4.04 \mathrm{dd}(11.9,6.2)$ \\
\hline 4 & & - & - \\
\hline 5 & $2.00 \mathrm{~d}(12.6)$ & $2.01 \mathrm{~d}(13.0)$ & $2.00 \mathrm{~d}(13.9)$ \\
\hline 6 & $\begin{array}{c}2.15 \mathrm{~d}(13.1), 1.60 \mathrm{dd}(12.9 \\
6.4)\end{array}$ & $\begin{array}{c}2.14 \mathrm{~d}(12.8) ; 1.61 \mathrm{dd}(12.8, \\
6.3)\end{array}$ & $2.14 \mathrm{~d}(13.2) ; 1.61 \mathrm{~m}$ \\
\hline 7 & $3.14 \mathrm{~m}$ & $3.14 \mathrm{~m}$ & $3.14 \mathrm{~m}$ \\
\hline 8 & - & - & - \\
\hline 9 & - & - & - \\
\hline 10 & - & - & - \\
\hline 11 & $2.29 \mathrm{~m}, 2.20 \mathrm{~m}$ & $\begin{array}{c}2.27 \mathrm{~m} ; 2.21 \mathrm{ddd}(9.1,6.3 \\
2.9)\end{array}$ & $\begin{array}{c}2.27 \mathrm{~m} ; 2.21 \mathrm{ddd}(9.0,6.3, \\
2.7)\end{array}$ \\
\hline 12 & $1.80 \mathrm{~m}, 1.76 \mathrm{~m}$ & $1.80 \mathrm{ov} ; 1.77 \mathrm{ov}$ & $1.80 \mathrm{~m} ; 1.77 \mathrm{~m}$ \\
\hline 13 & - & - & - \\
\hline 14 & - & - & - \\
\hline 15 & $\begin{array}{l}2.53 \text { ddd }(16.3,6.1,2.4), \\
2.36 \text { ddd }(15.9,11.7,3.5)\end{array}$ & $2.54 \mathrm{~m} ; 2.35 \mathrm{~m}$ & $\begin{array}{c}2.53 \text { ddd }(16.3,6.2,2.2) ; \\
2.35 \mathrm{~m}\end{array}$ \\
\hline 16 & $1.68 \mathrm{~m}(2 \mathrm{D}), 1.66 \mathrm{~m}$ & $1.68 \mathrm{ov} ; 1.65 \mathrm{ov}$ & $1.68 \mathrm{~m} ; 1.65 \mathrm{~m}$ \\
\hline 17 & $1.26 \mathrm{~m}$ & $1.25 \mathrm{ov}$ & $1.25 \mathrm{~m}$ \\
\hline 18 & $1.01 \mathrm{~s}$ & $1.00 \mathrm{~s}$ & $1.01 \mathrm{~s}$ \\
\hline 19 & $0.78 \mathrm{~s}$ & $0.78 \mathrm{~s}$ & $0.78 \mathrm{~s}$ \\
\hline 20 & $1.56 \mathrm{~m}$ & $1.56 \mathrm{ov}$ & $1.59 \mathrm{~m}$ \\
\hline 21 & $0.93 d(6.9)$ & $0.92 \mathrm{~d}(6.8)$ & $0.93 d(6.9)$ \\
\hline 22 & $0.91 \mathrm{~m} ; 1.39 \mathrm{~m}$ & $0.91 \mathrm{~m} ; 1.39 \mathrm{ov}$ & $0.91 \mathrm{~m} ; 1.37 \mathrm{~m}$ \\
\hline 23 & $1.19 \mathrm{~m}$ & $1.19 \mathrm{ov}$ & $1.19 \mathrm{~m}$ \\
\hline 24 & $1.20 \mathrm{~m}$ & $1.20 \mathrm{ov}$ & $1.20 \mathrm{~m}$ \\
\hline 241 & $0.78 d(6.5)$ & $0.78 d(6.8)$ & 0.78 \\
\hline 25 & $1.55 \mathrm{~m}$ & $1.55 \mathrm{ov}$ & $1.55 \mathrm{~m}$ \\
\hline 26 & $0.86 d(6.8)$ & $0.85 d(6.8)$ & $0.85 d(6.8)$ \\
\hline 27 & $0.80 \mathrm{~d}(6.8)$ & $0.80 \mathrm{~d}(7.1)$ & 0.79 \\
\hline 28 & $5.09 \mathrm{~s} ; 4.69 \mathrm{~s}$ & $5.09 \mathrm{~s} ; 4.68 \mathrm{~s}$ & $5.09 \mathrm{~s} ; 4.69 \mathrm{~s}$ \\
\hline
\end{tabular}

All chemical shifts are reported in ppm. Coupling constants are in parentheses and are reported in $\mathrm{Hz} . \mathrm{m}=$ centered multiplet, ov $=$ overlain. All spectra were measured in $\mathrm{CDCl}_{3}$ and are referenced to the residual solvent peak at $\delta_{\mathrm{H}}=7.26 \mathrm{ppm}$. ${ }^{\text {a }}$ Recorded at $700 \mathrm{MHz}$. ${ }^{\mathrm{b}}$ recorded at $500 \mathrm{MHz}$. 
Table S5. ${ }^{13} \mathrm{C}-\mathrm{NMR}$ comparison of synthetic swinhoeisterol A, natural swinhoeisterol A (1) ${ }^{[6]}$ and synthetic 24-epi-swinhoeisterol A (24-epi-1).

\begin{tabular}{|c|c|c|c|}
\hline$\#$ & Synthetica & Natural[6],b & Synthetic 24-epia \\
\hline 1 & 34.8 & 34.7 & 34.8 \\
\hline 2 & 32.5 & 32.4 & 32.5 \\
\hline 3 & 73.1 & 73.1 & 73.1 \\
\hline 4 & 151.9 & 151.9 & 151.9 \\
\hline 5 & 44.4 & 44.3 & 44.4 \\
\hline 6 & 24.5 & 24.5 & 24.5 \\
\hline 7 & 45.3 & 45.3 & 45.3 \\
\hline 8 & 136.0 & 135.9 & 135.9 \\
\hline 9 & 146.9 & 146.9 & 146.9 \\
\hline 10 & 37.7 & 37.7 & 37.7 \\
\hline 11 & 27.8 & 27.8 & 27.8 \\
\hline 12 & 38.7 & 38.7 & 38.7 \\
\hline 13 & 53.8 & 53.8 & 53.8 \\
\hline 14 & 213.6 & 213.7 & 213.6 \\
\hline 15 & 45.4 & 45.4 & 45.4 \\
\hline 16 & 20.1 & 20.1 & 20.1 \\
\hline 17 & 55.8 & 55.8 & 55.9 \\
\hline 18 & 20.0 & 20.0 & 20.0 \\
\hline 19 & 17.9 & 17.9 & 17.9 \\
\hline 20 & 35.2 & 35.1 & 35.5 \\
\hline 21 & 21.6 & 21.6 & 21.7 \\
\hline 22 & 30.5 & 30.5 & 30.7 \\
\hline 23 & 33.5 & 33.4 & 33.7 \\
\hline 24 & 39.1 & 39.1 & 39.2 \\
\hline 241 & 15.6 & 15.6 & 15.6 \\
\hline 25 & 32.2 & 32.3 & 31.9 \\
\hline 26 & 20.4 & 20.4 & 20.5 \\
\hline 27 & 18.2 & 18.2 & 17.9 \\
\hline 28 & 102.9 & 102.9 & 102.9 \\
\hline
\end{tabular}

All chemical shifts are reported in ppm. All spectra were measured in $\mathrm{CDCl}_{3}$ and are referenced to the residual solvent peak at $\delta_{\mathrm{C}}=77.16 \mathrm{ppm}$. a Recorded at $176 \mathrm{MHz}$. b recorded at $126 \mathrm{MHz}$. Signals that are shifted due to different configuration at $\mathrm{C} 24$ are marked in light grey. 


\section{NMR Spectra}

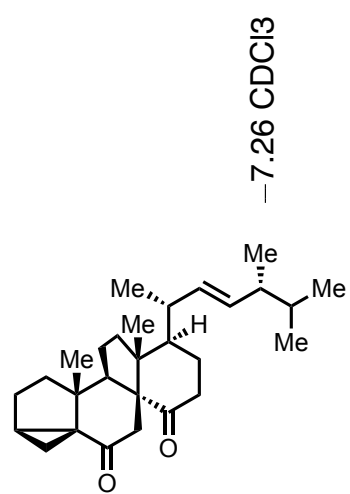

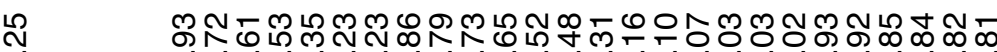

7; ${ }^{1} \mathrm{H}-\mathrm{NMR}\left(700 \mathrm{MHz}, \mathrm{CDCl}_{3}\right)$

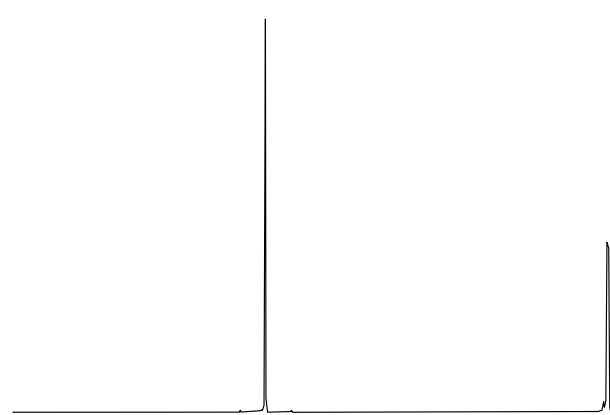

年

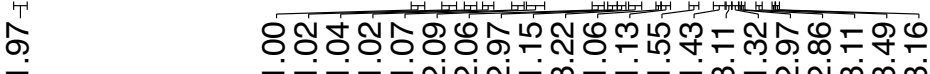

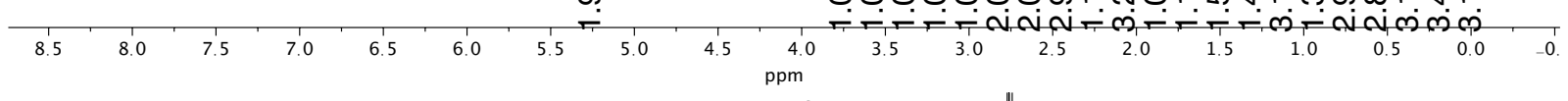

Lִ

는

ปู

芦官

nั.

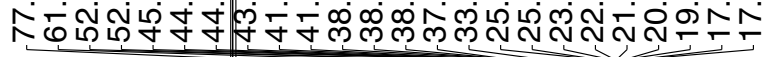

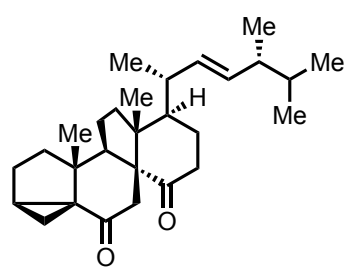

7; ${ }^{13} \mathrm{C}-\mathrm{NMR}\left(176 \mathrm{MHz}, \mathrm{CDCl}_{3}\right)$

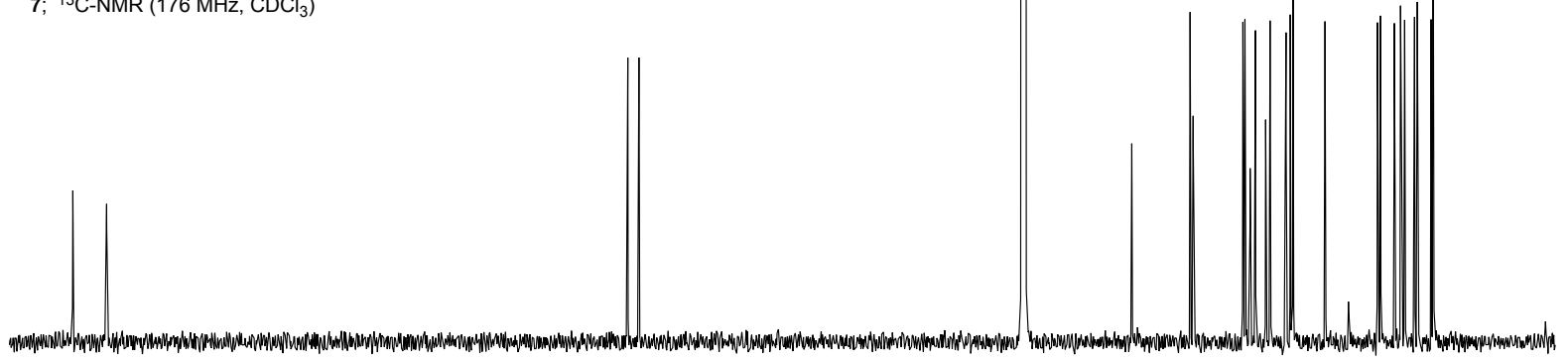

$220-210-200 \quad 190-180-170-160-150-140$

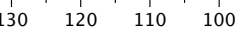

$90 \quad 80$

$70 \quad 60$

50

$\begin{array}{llll}1 & 1 & 1 & 1 \\ 30 & 20 & 10 & 0\end{array}$ 

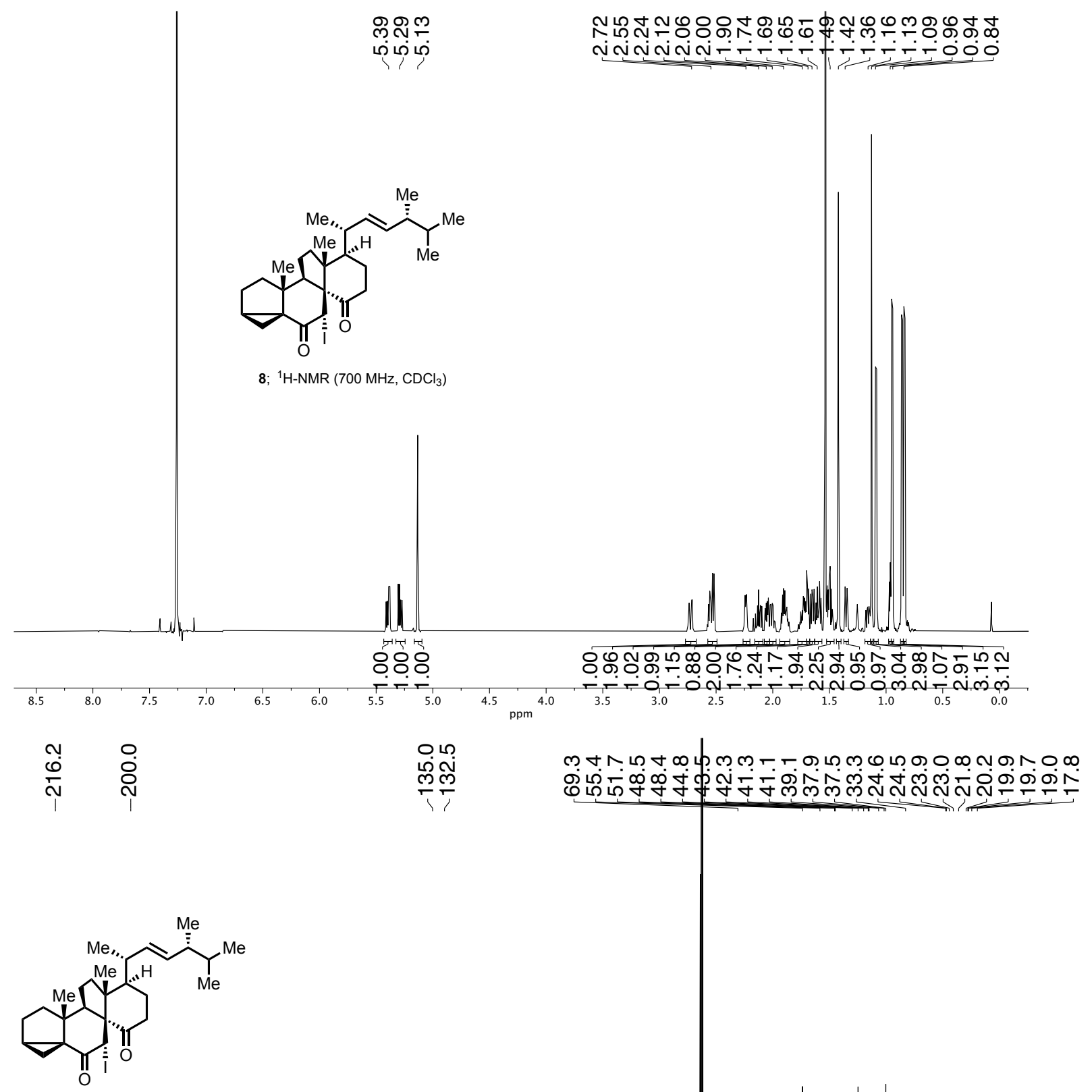

8; ${ }^{13} \mathrm{C}-\mathrm{NMR}\left(176 \mathrm{MHz}, \mathrm{CDCl}_{3}\right)$

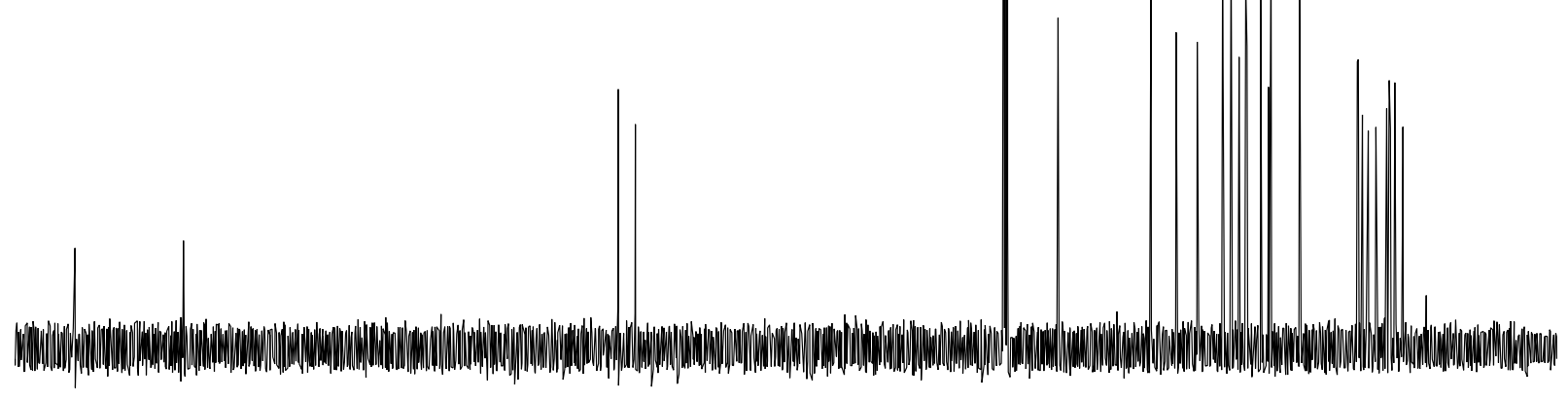

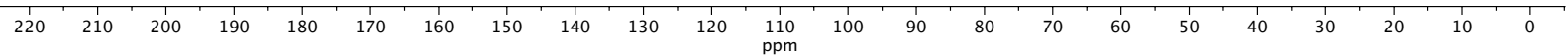




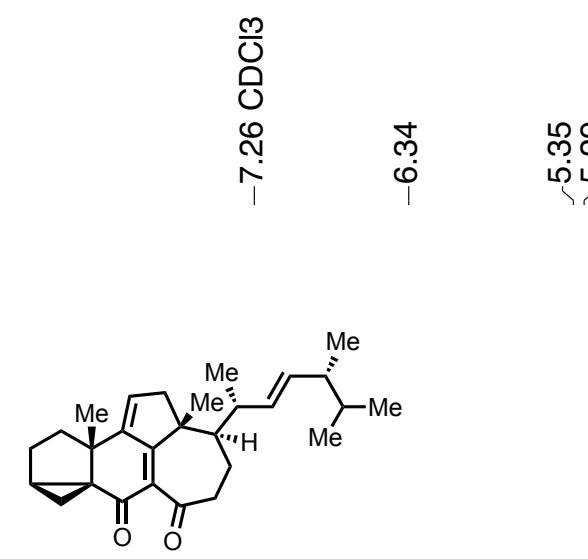

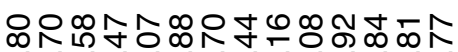

กิ

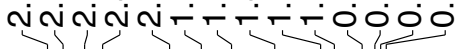

9; ${ }^{1} \mathrm{H}-\mathrm{NMR}\left(500 \mathrm{MHz}, \mathrm{CDCl}_{3}\right)$

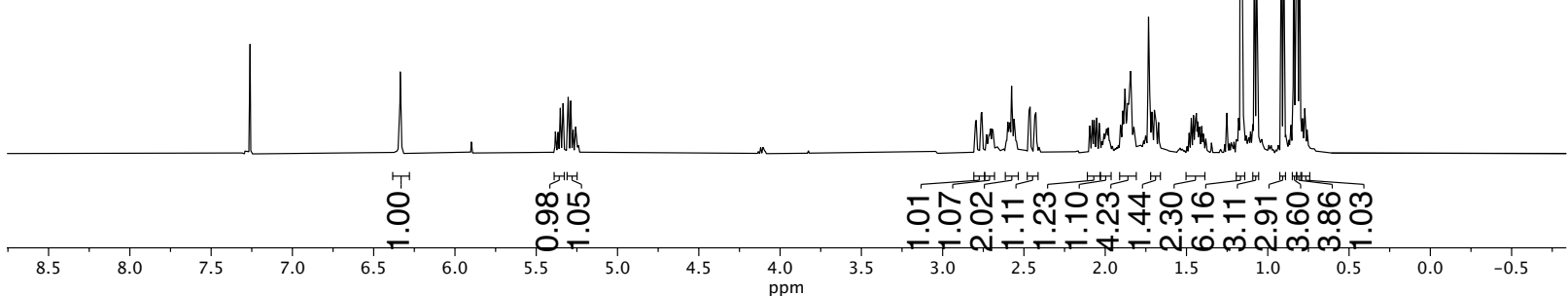

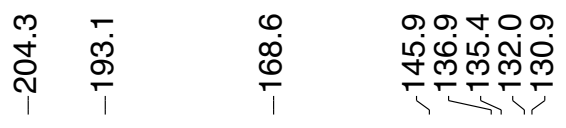

r.

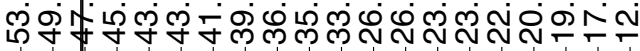

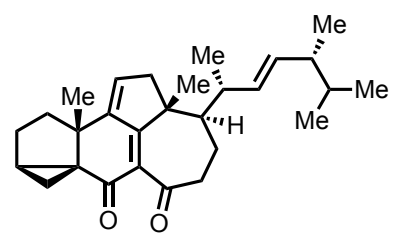

9; ${ }^{13} \mathrm{C}-\mathrm{NMR}\left(126 \mathrm{MHz}, \mathrm{CDCl}_{3}\right)$

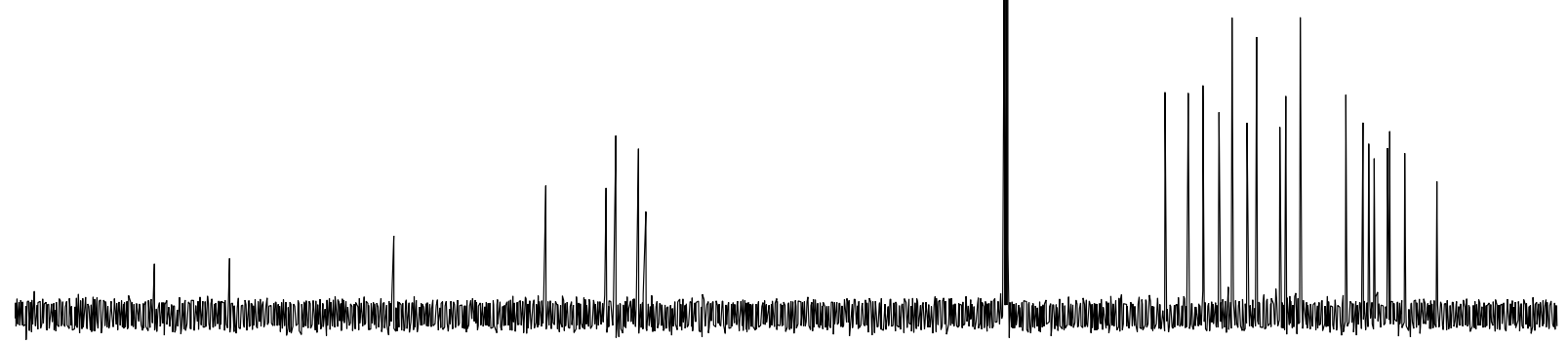

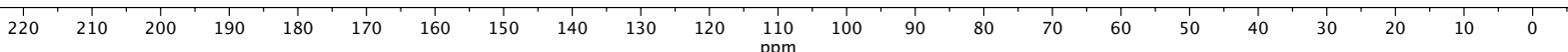




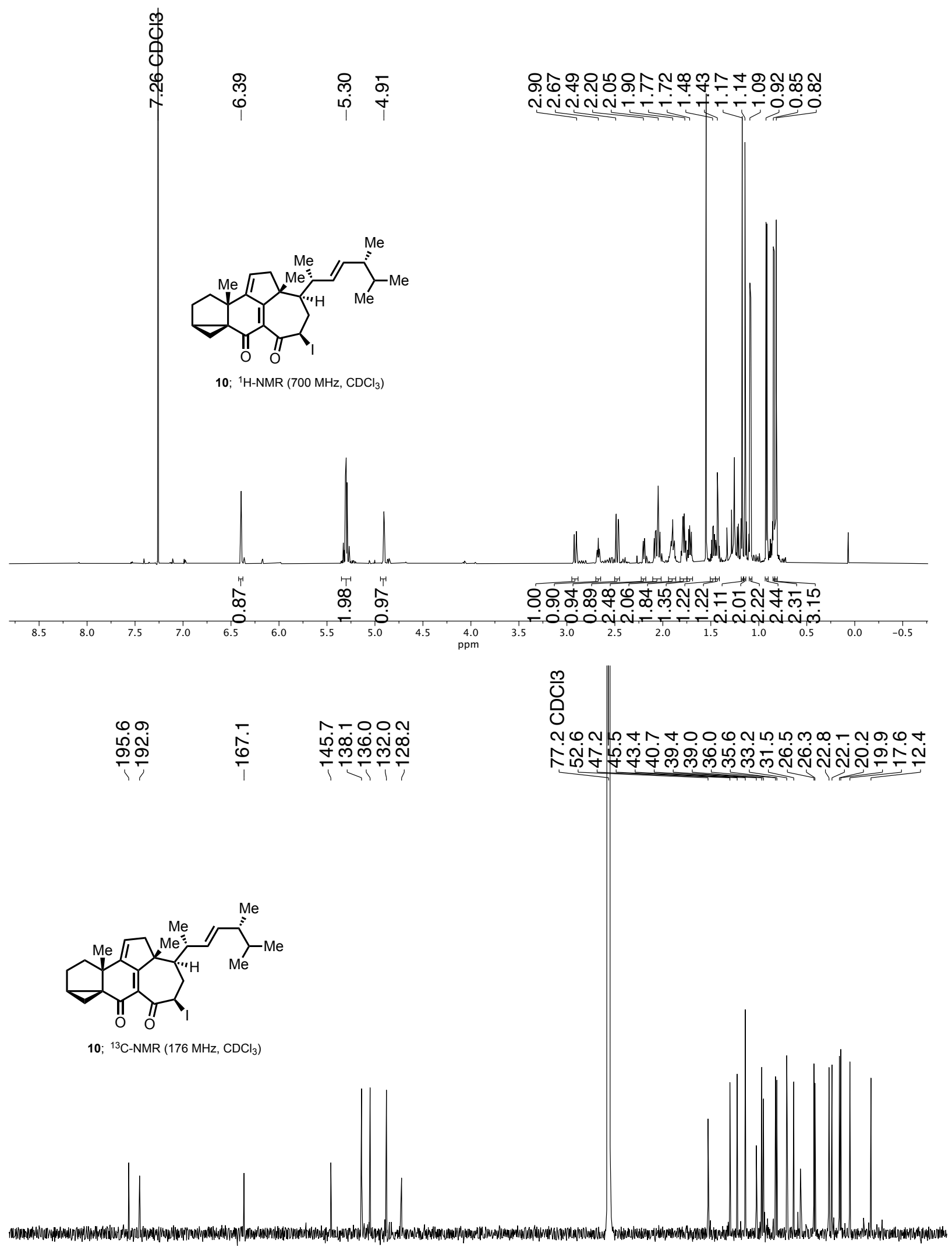

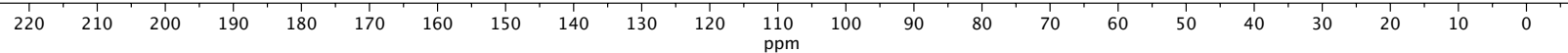




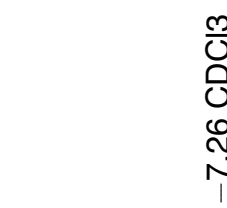

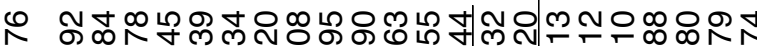

i

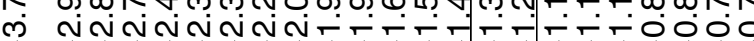

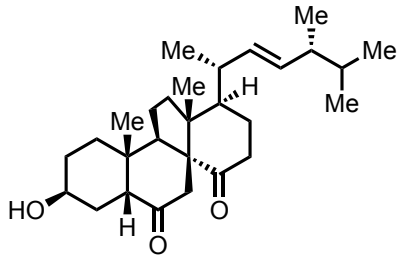

11; ${ }^{1} \mathrm{H}-\mathrm{NMR}\left(700 \mathrm{MHz}, \mathrm{CDCl}_{3}\right)$
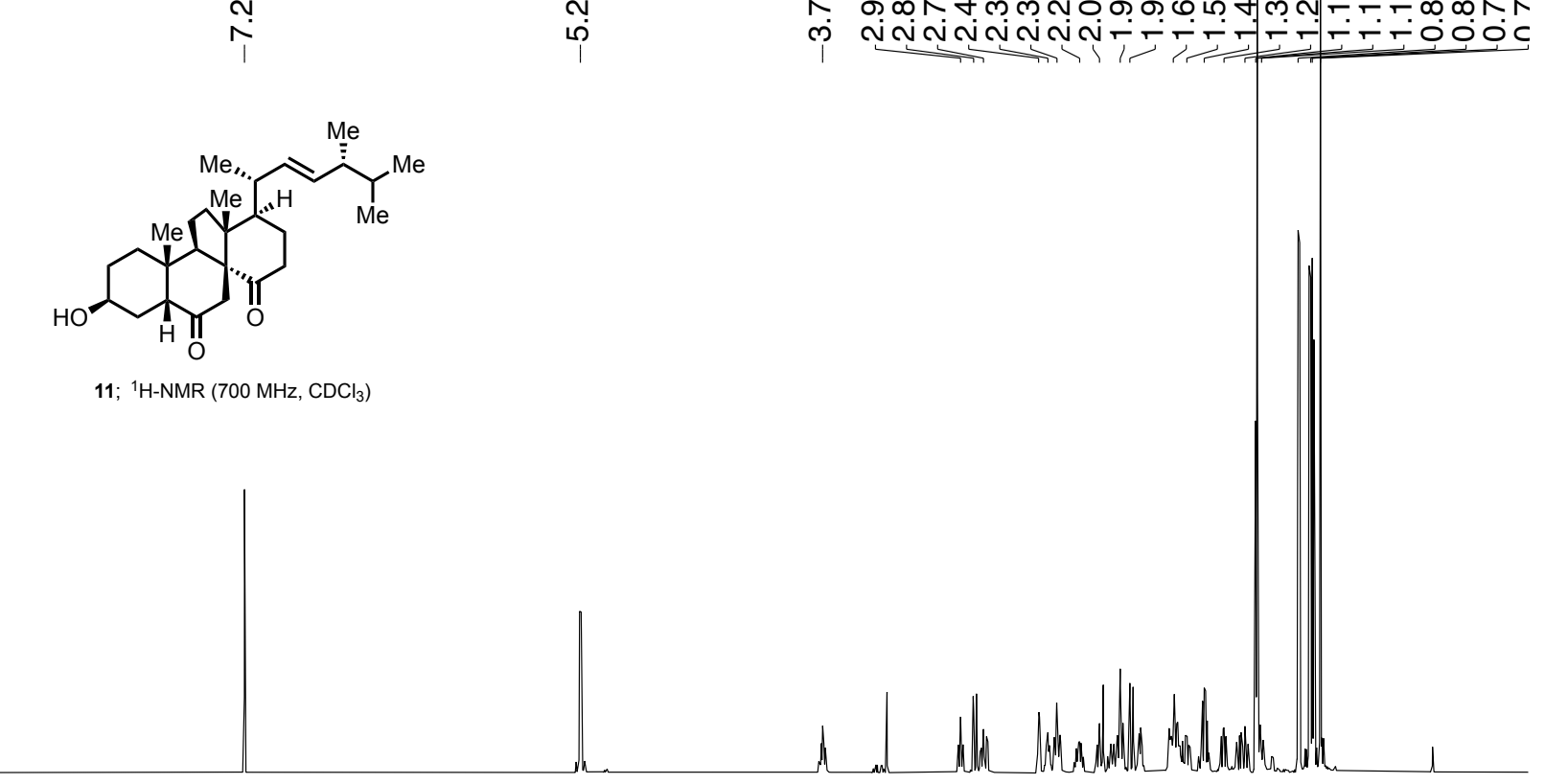

की

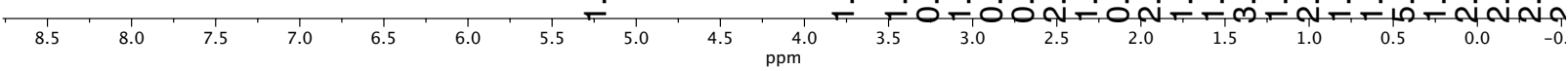
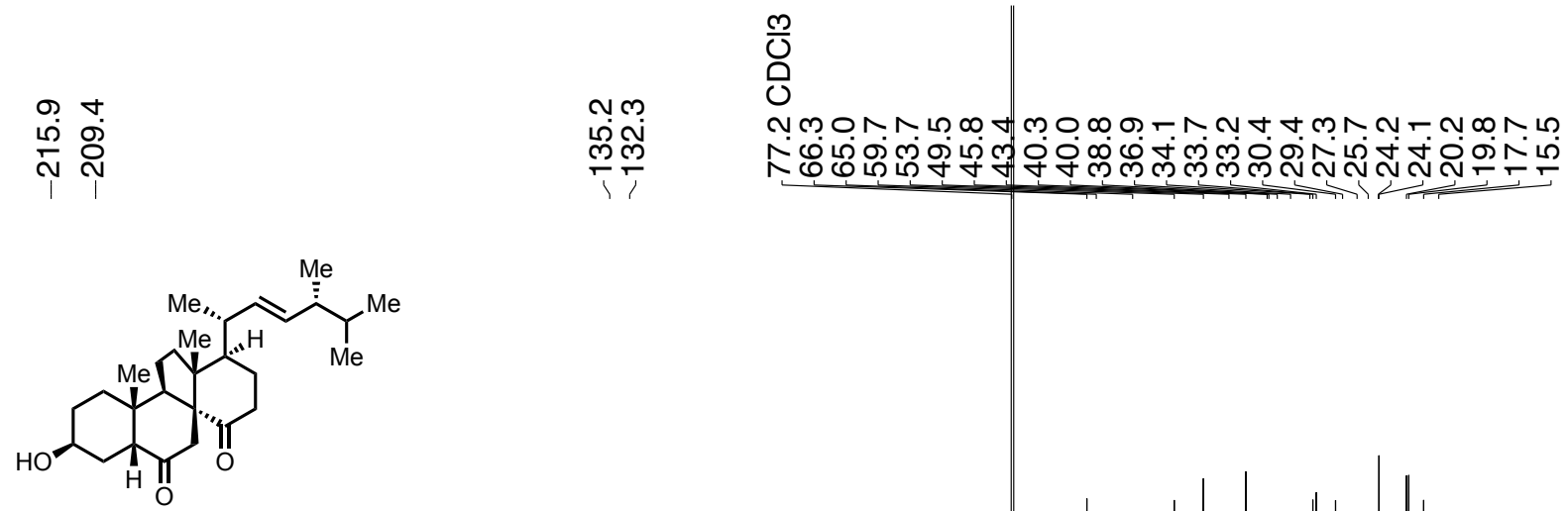

11; ${ }^{13} \mathrm{C}-\mathrm{NMR}\left(176 \mathrm{MHz}, \mathrm{CDCl}_{3}\right)$

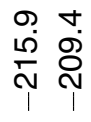

กิ กิ

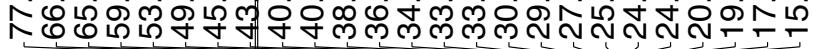

,

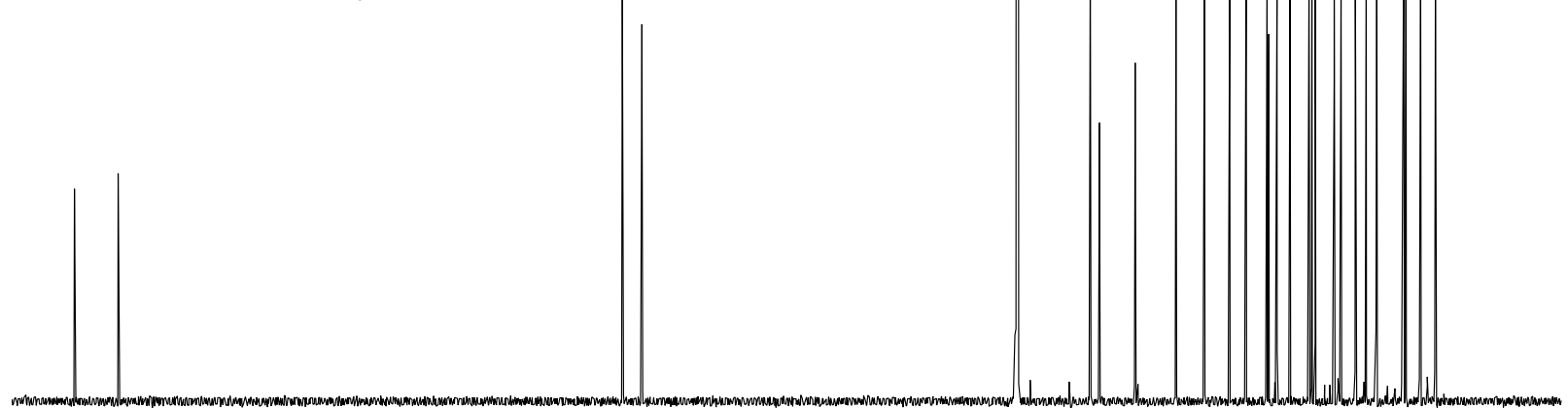

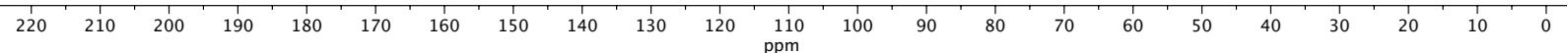




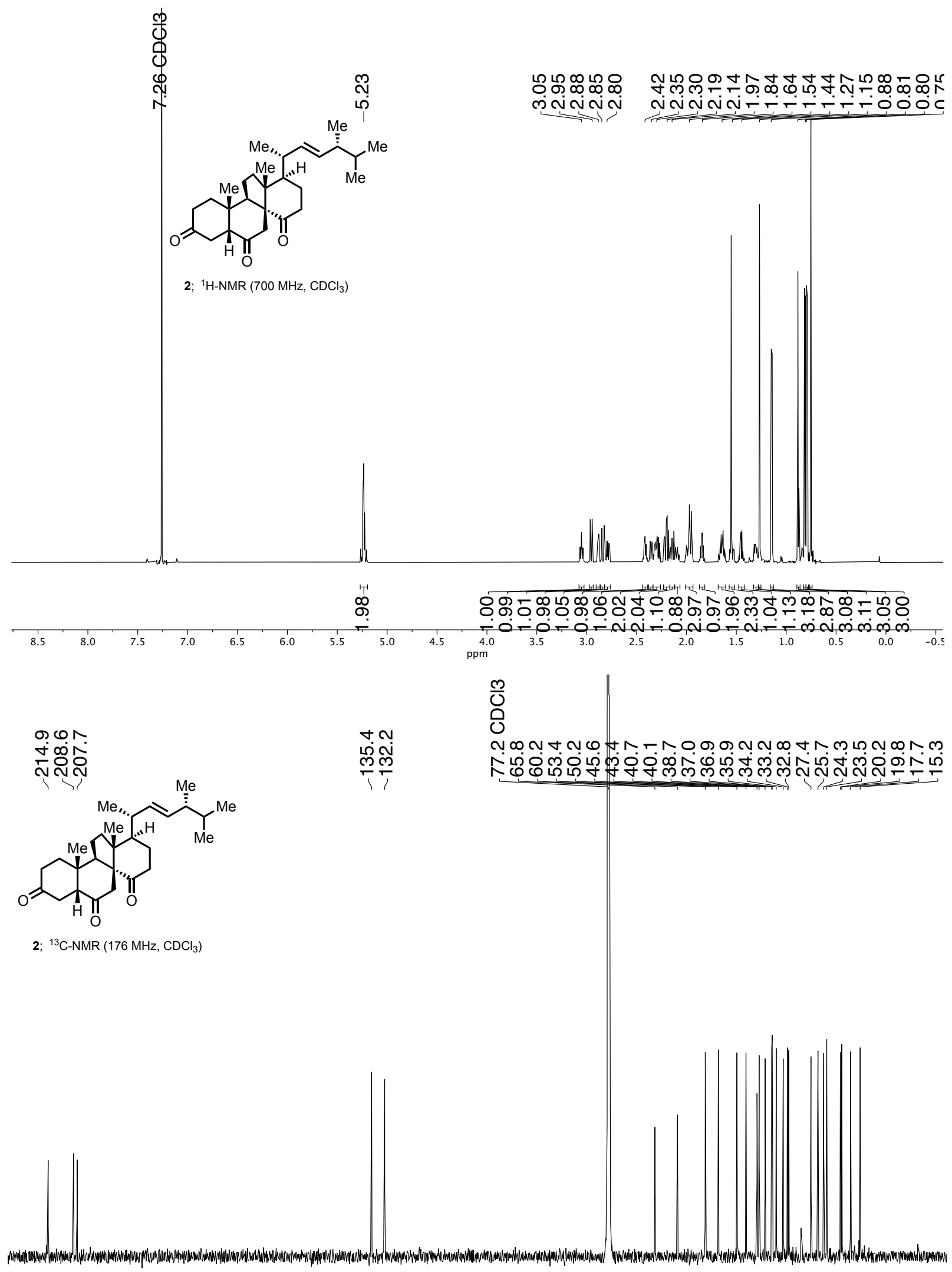

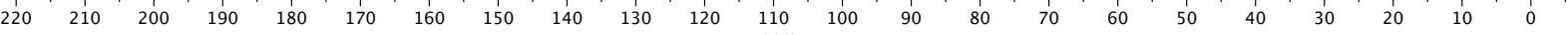



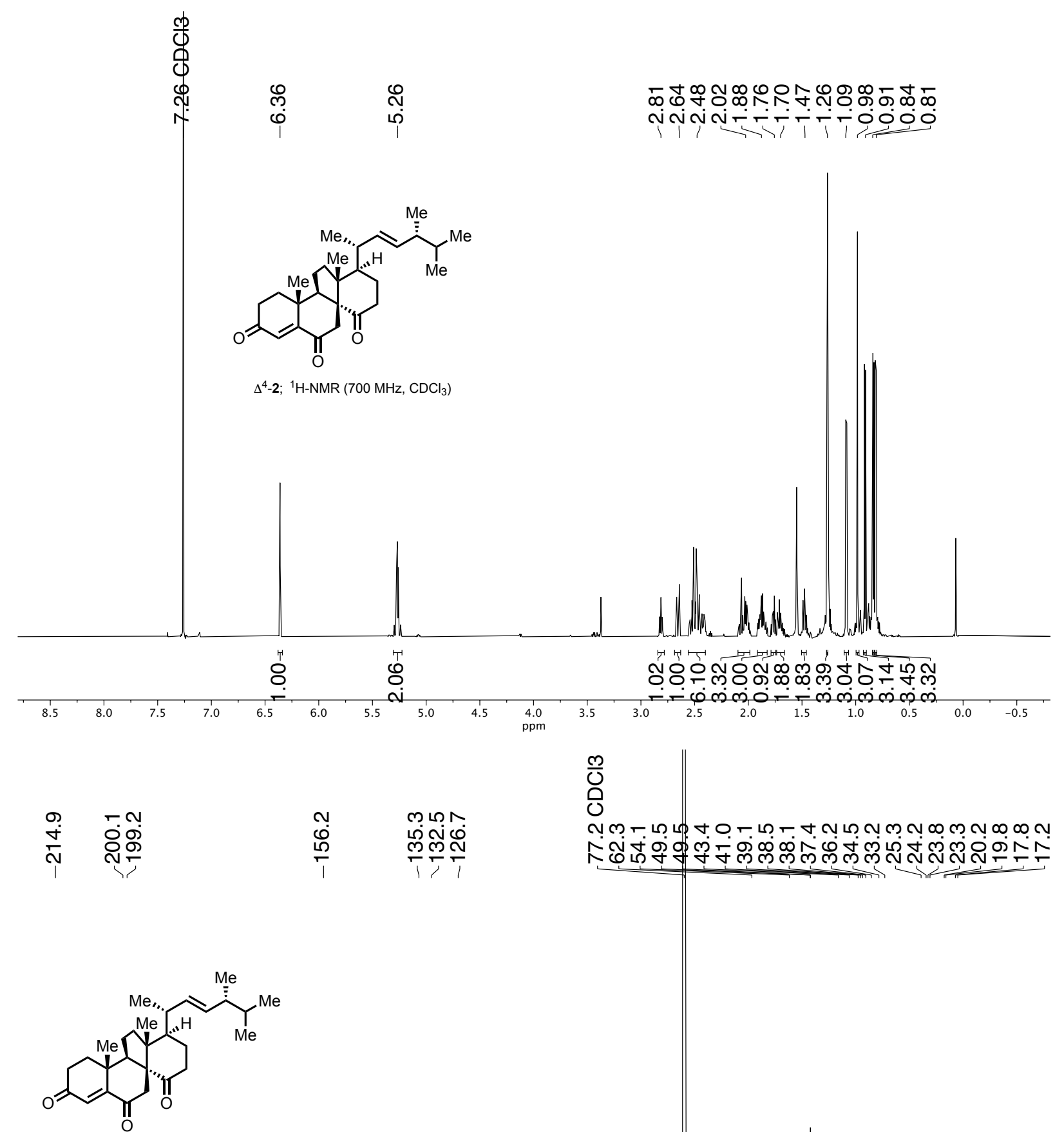

$\Delta^{4}-2 ;{ }^{13} \mathrm{C}-\mathrm{NMR}\left(176 \mathrm{MHz}, \mathrm{CDCl}_{3}\right)$

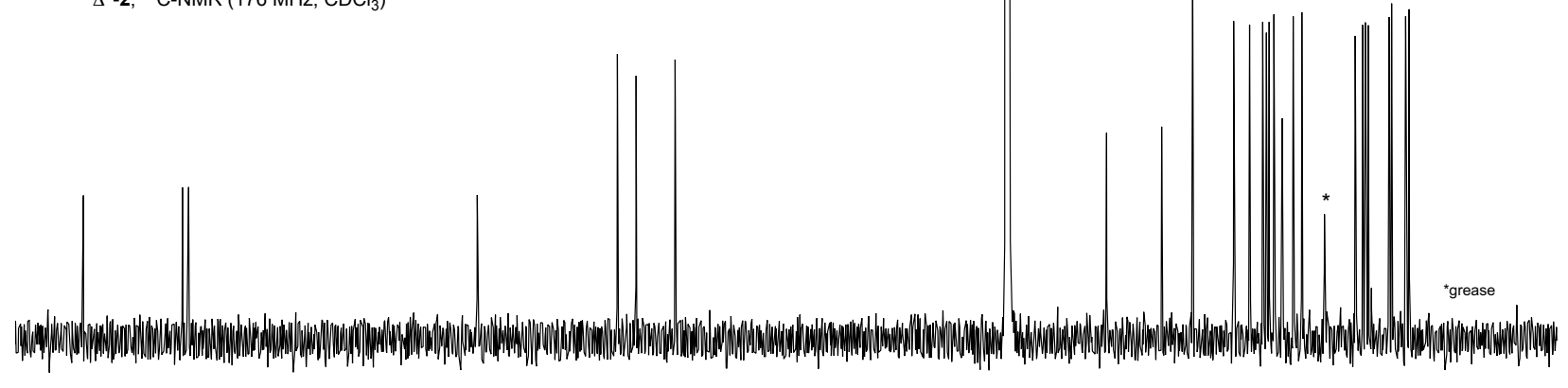

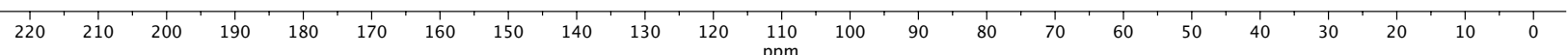



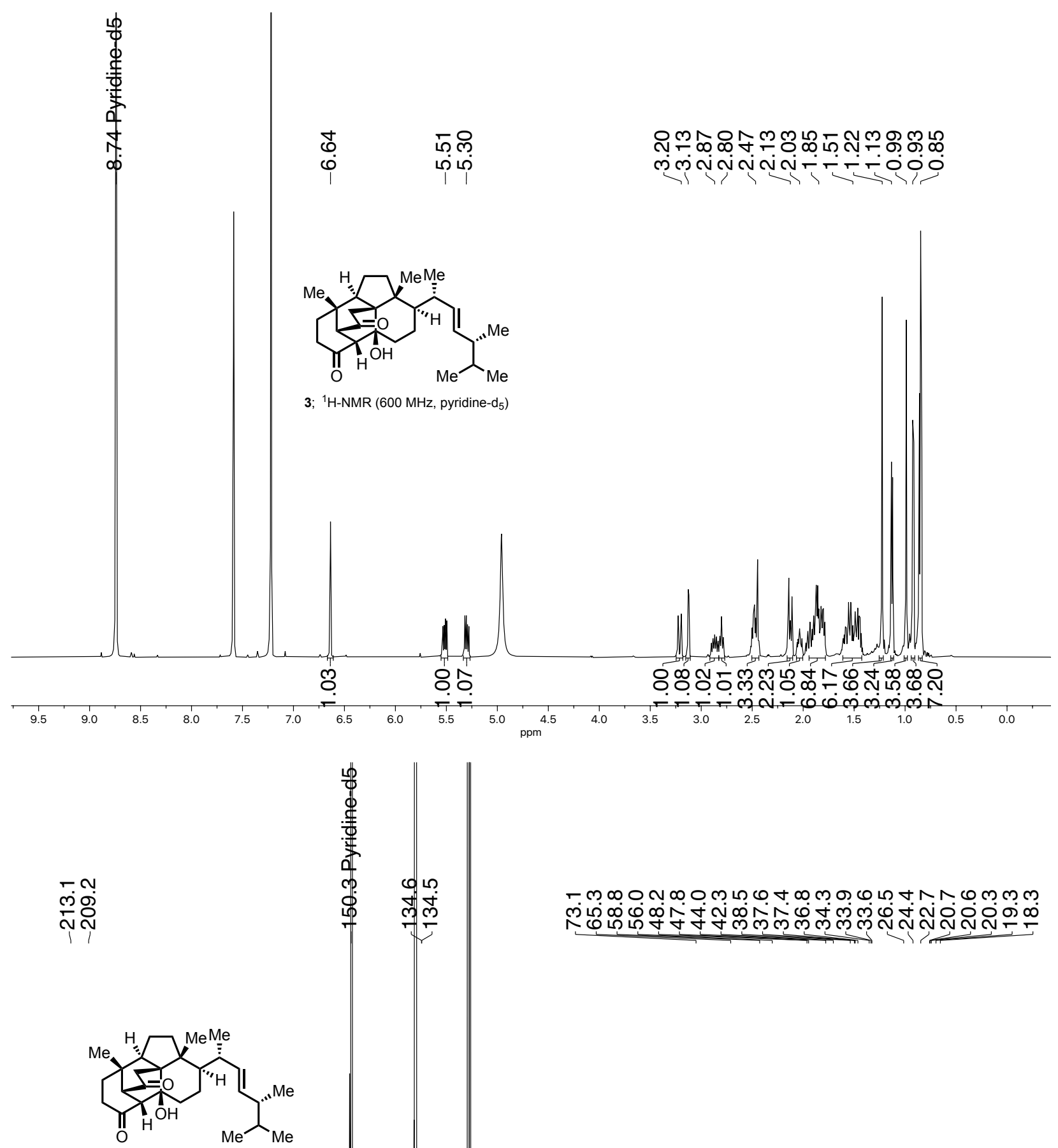

3; ${ }^{13} \mathrm{C}-\mathrm{NMR}\left(151 \mathrm{MHz}\right.$, pyridine- $\mathrm{d}_{5}$ )

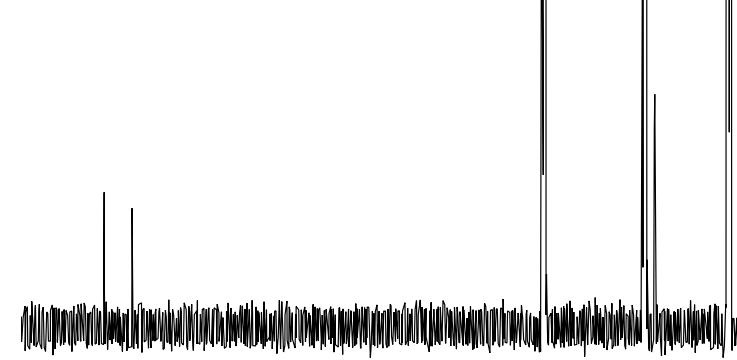

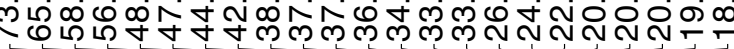



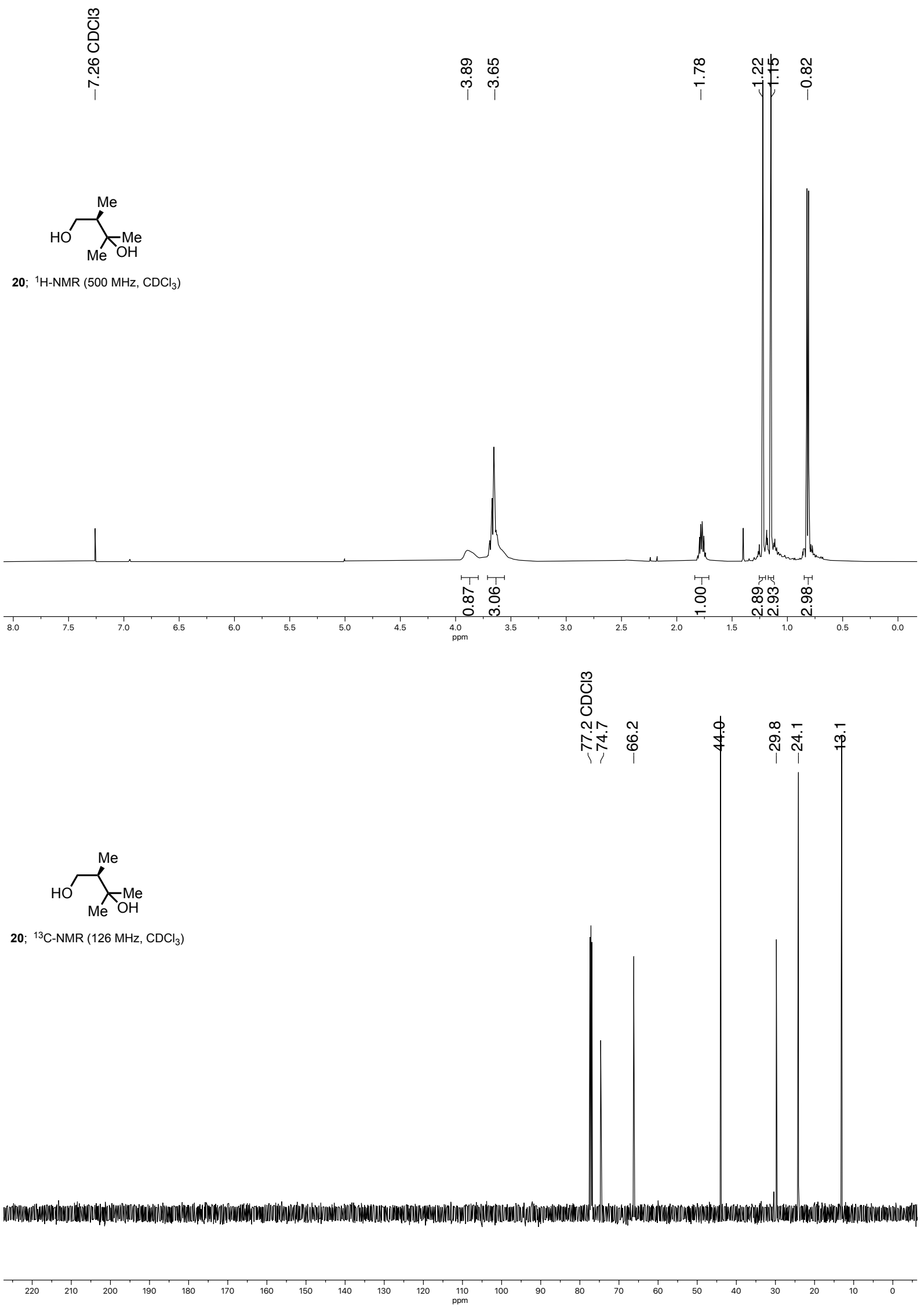


$$
\text { in }
$$

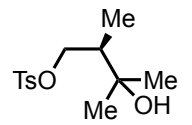$$
\text { ำ }
$$$$
\text { ఫ } \quad \stackrel{\infty}{\longleftarrow}
$$

S5; ${ }^{1} \mathrm{H}-\mathrm{NMR}\left(500 \mathrm{MHz}, \mathrm{CDCl}_{3}\right)$
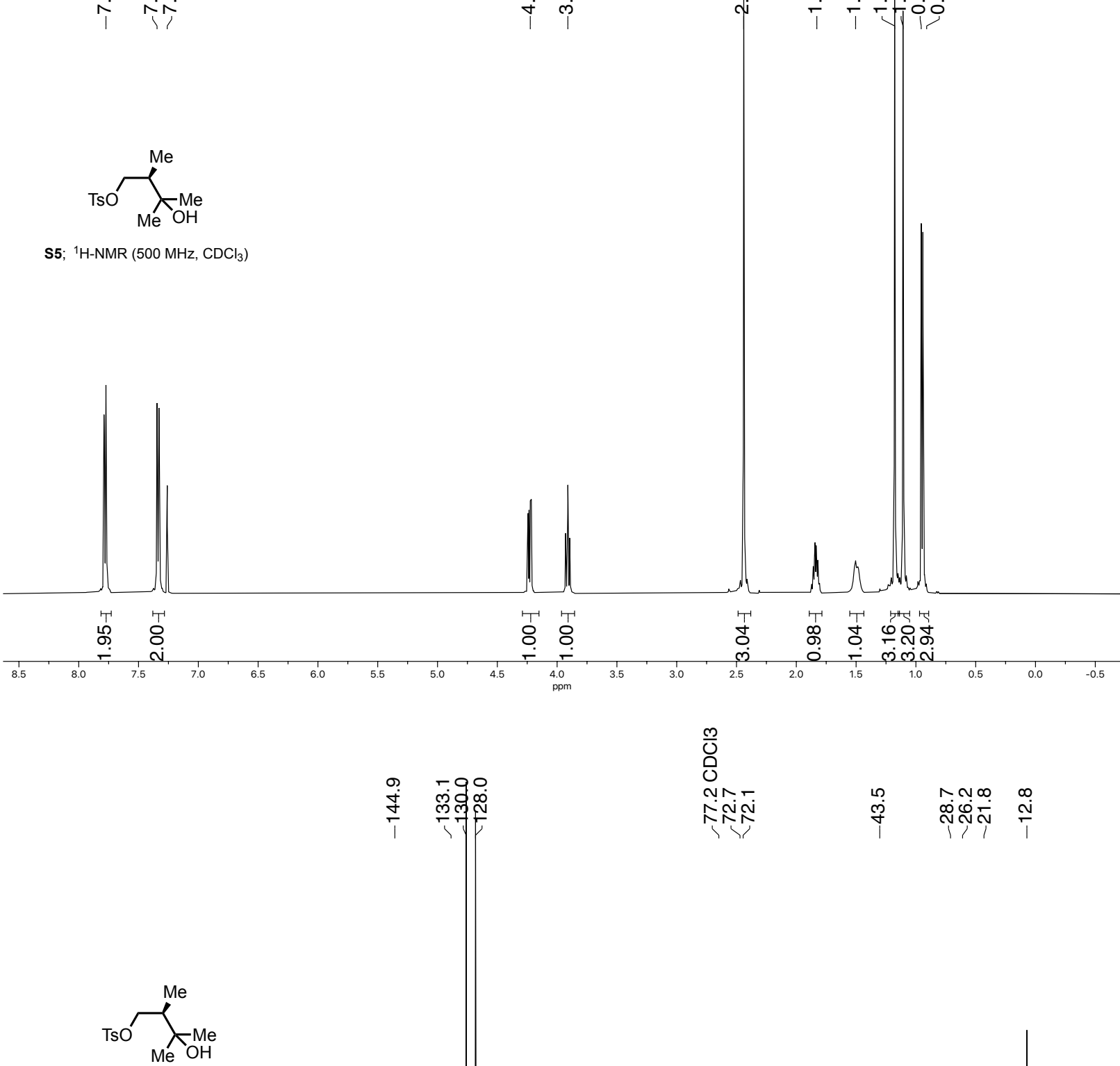

S5; ${ }^{13} \mathrm{C}-\mathrm{NMR}\left(126 \mathrm{MHz}, \mathrm{CDCl}_{3}\right)$
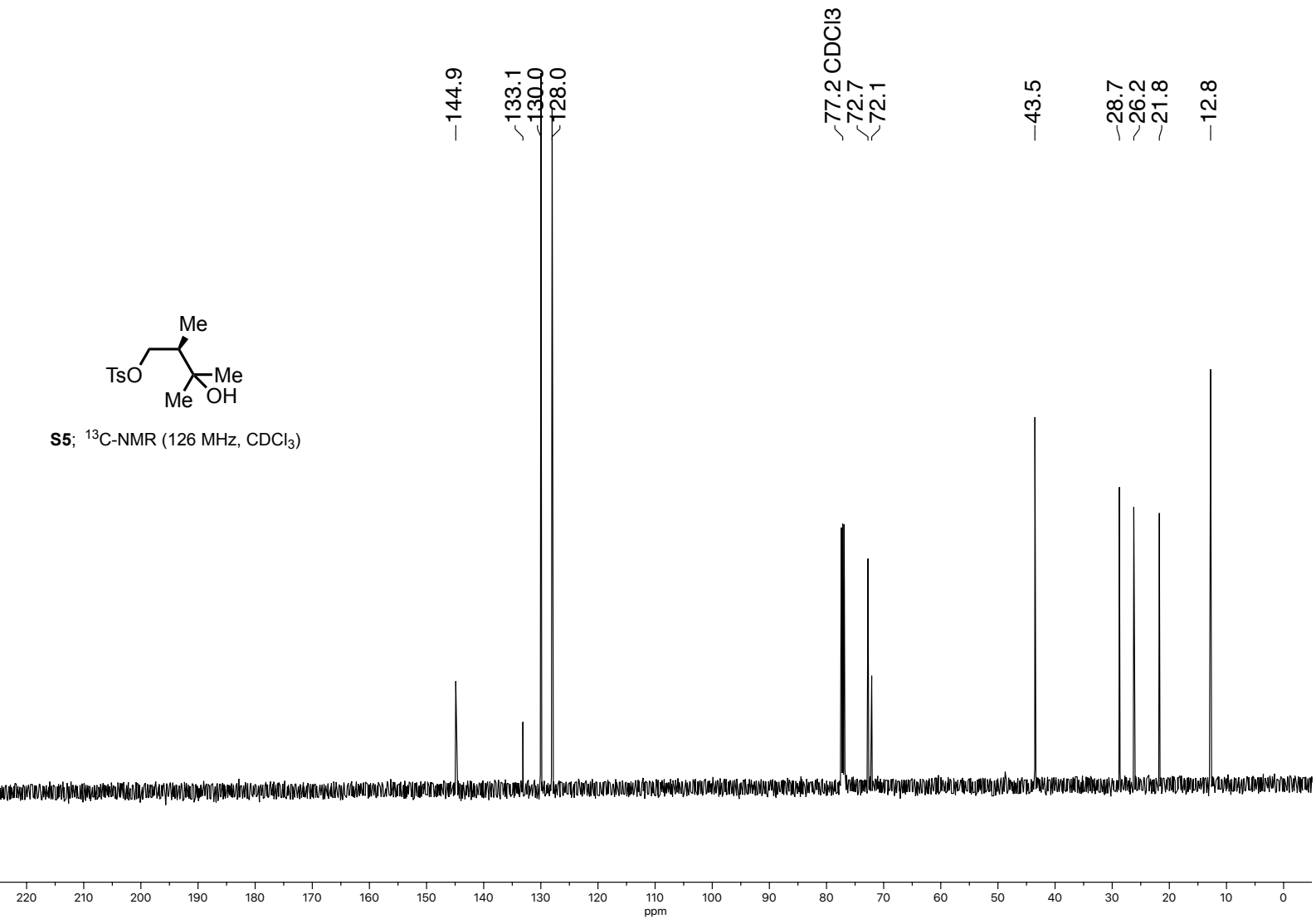


$$
\overbrace{M}^{M e}
$$

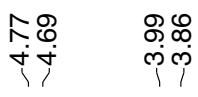

S6; ${ }^{1} \mathrm{H}-\mathrm{NMR}\left(500 \mathrm{MHz}, \mathrm{CDCl}_{3}\right)$
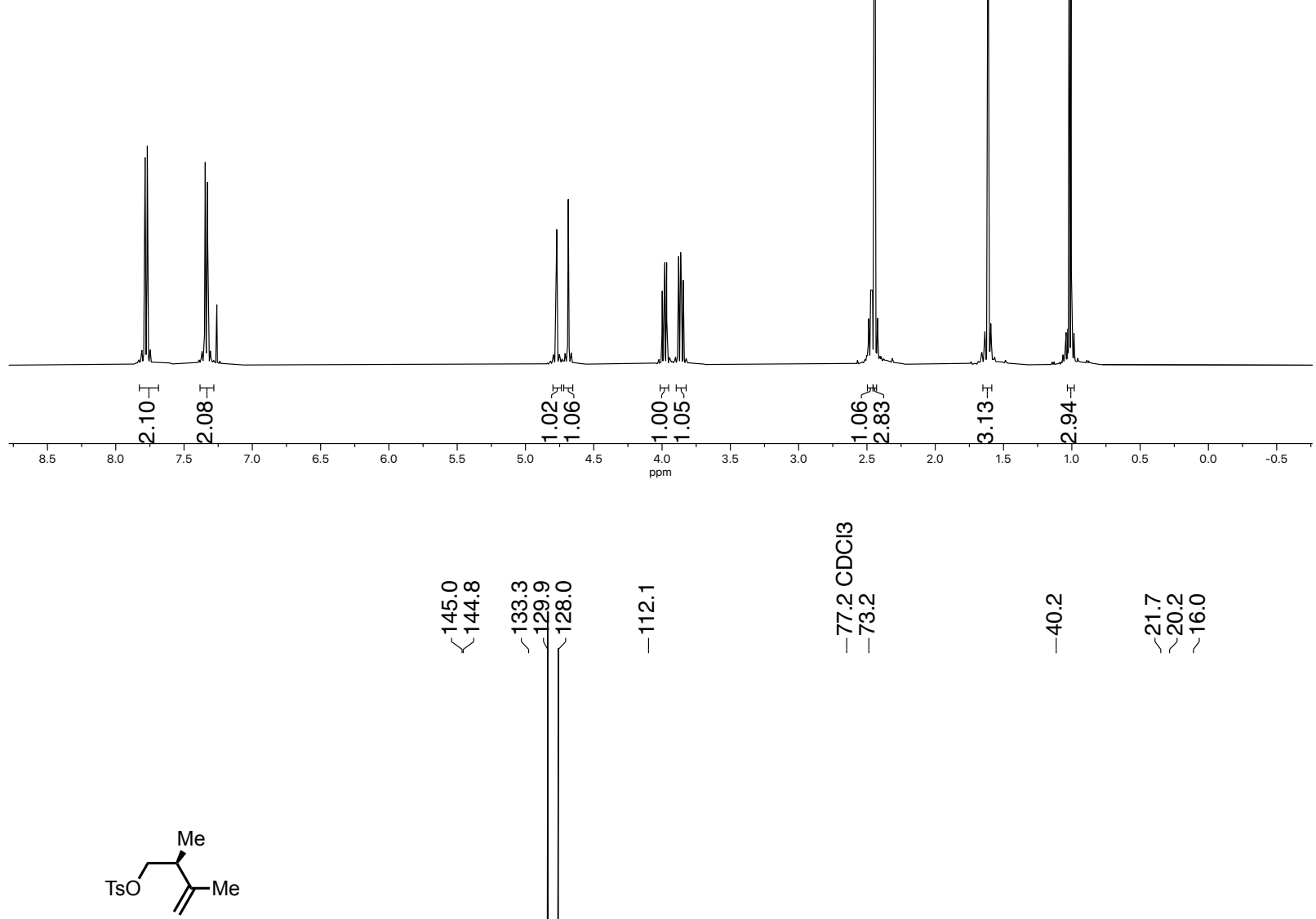

S6; ${ }^{13} \mathrm{C}-\mathrm{NMR}$ (126 MHz, $\mathrm{CDCl}_{3}$ )

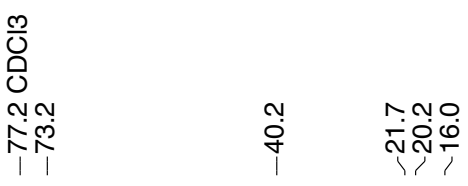

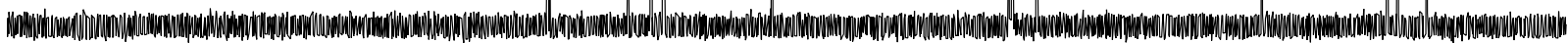

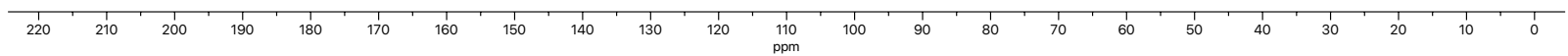




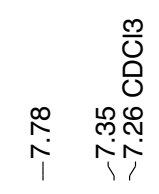

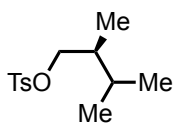

21; ${ }^{1} \mathrm{H}-\mathrm{NMR}\left(400 \mathrm{MHz}, \mathrm{CDCl}_{3}\right)$
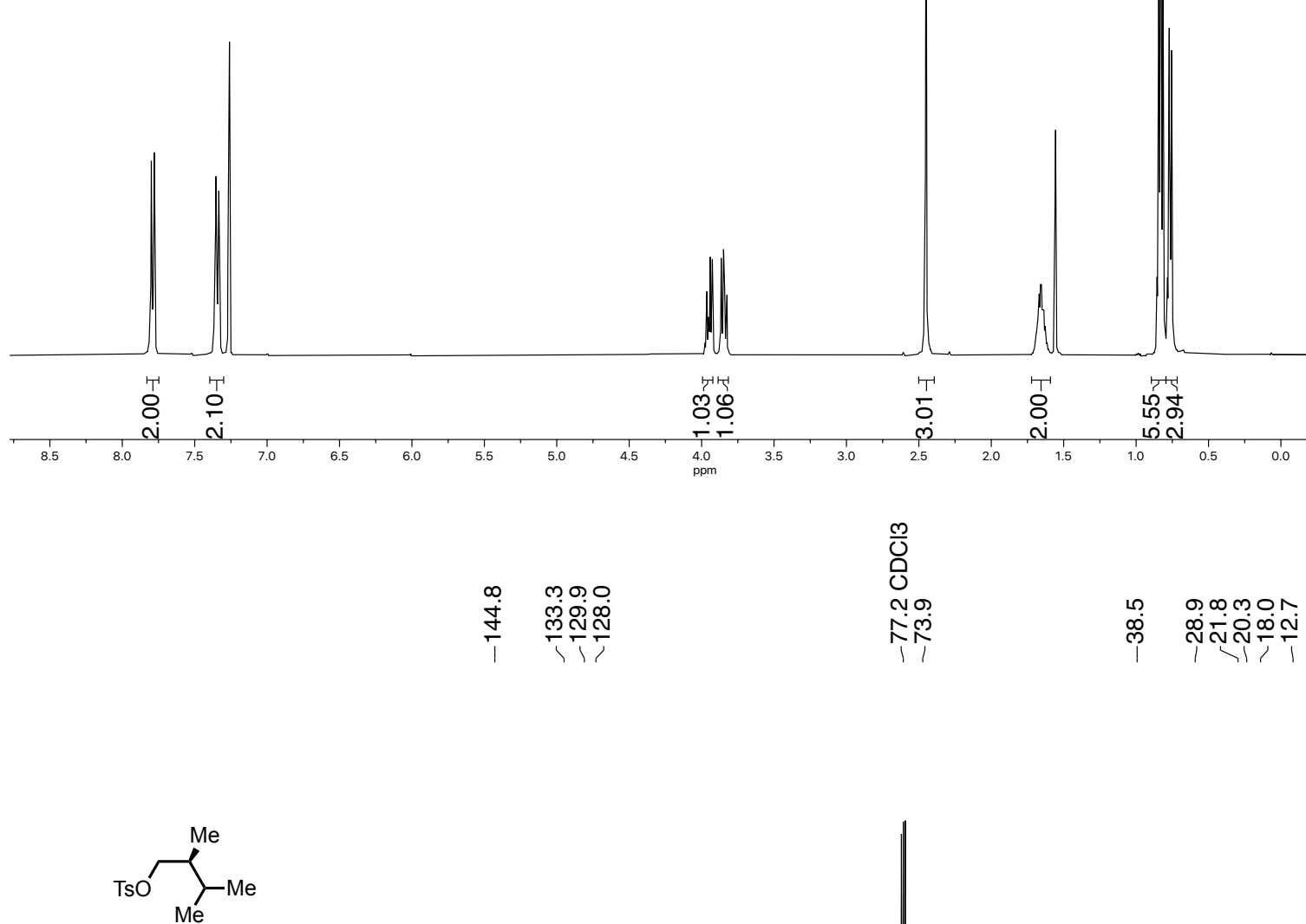

21; ${ }^{13} \mathrm{C}-\mathrm{NMR}\left(101 \mathrm{MHz}, \mathrm{CDCl}_{3}\right)$
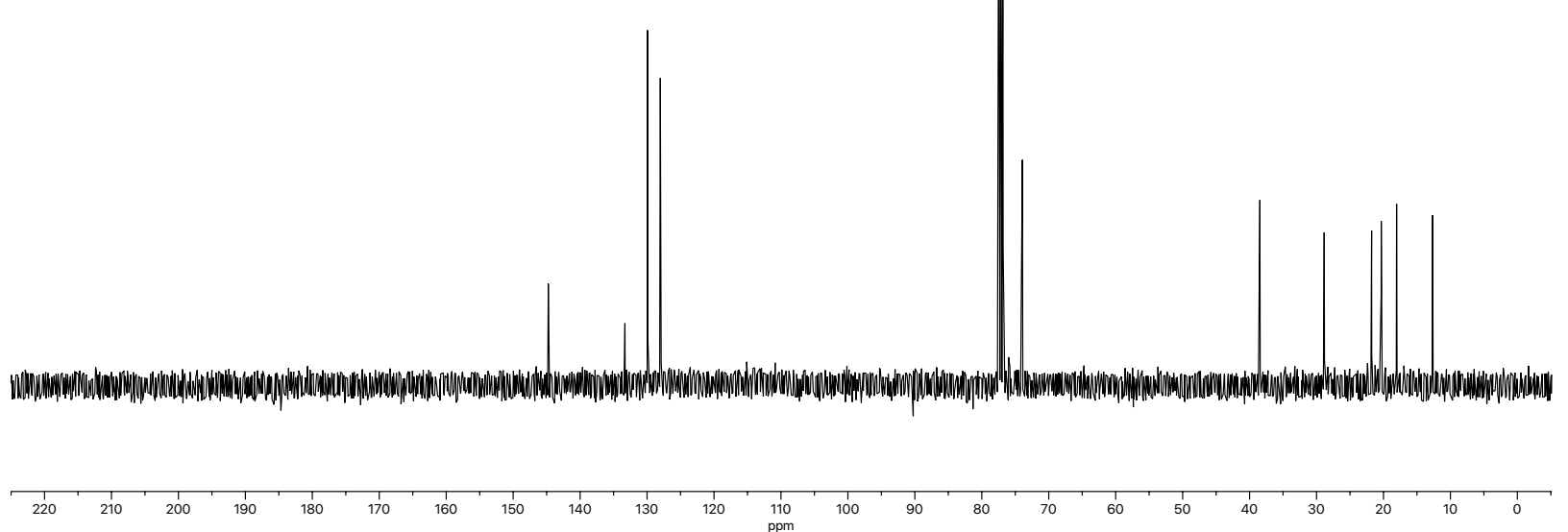


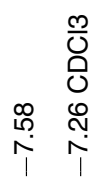

Гึ

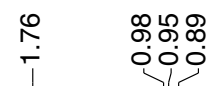

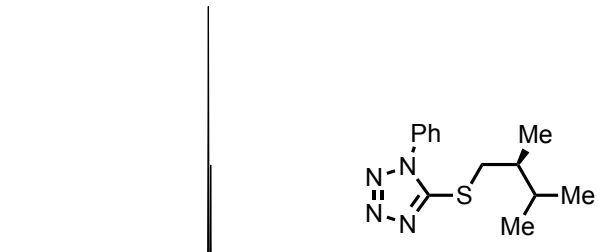

s7; ${ }^{1} \mathrm{H}-\mathrm{NMR}\left(500 \mathrm{MHz}, \mathrm{CDCl}_{3}\right.$ )
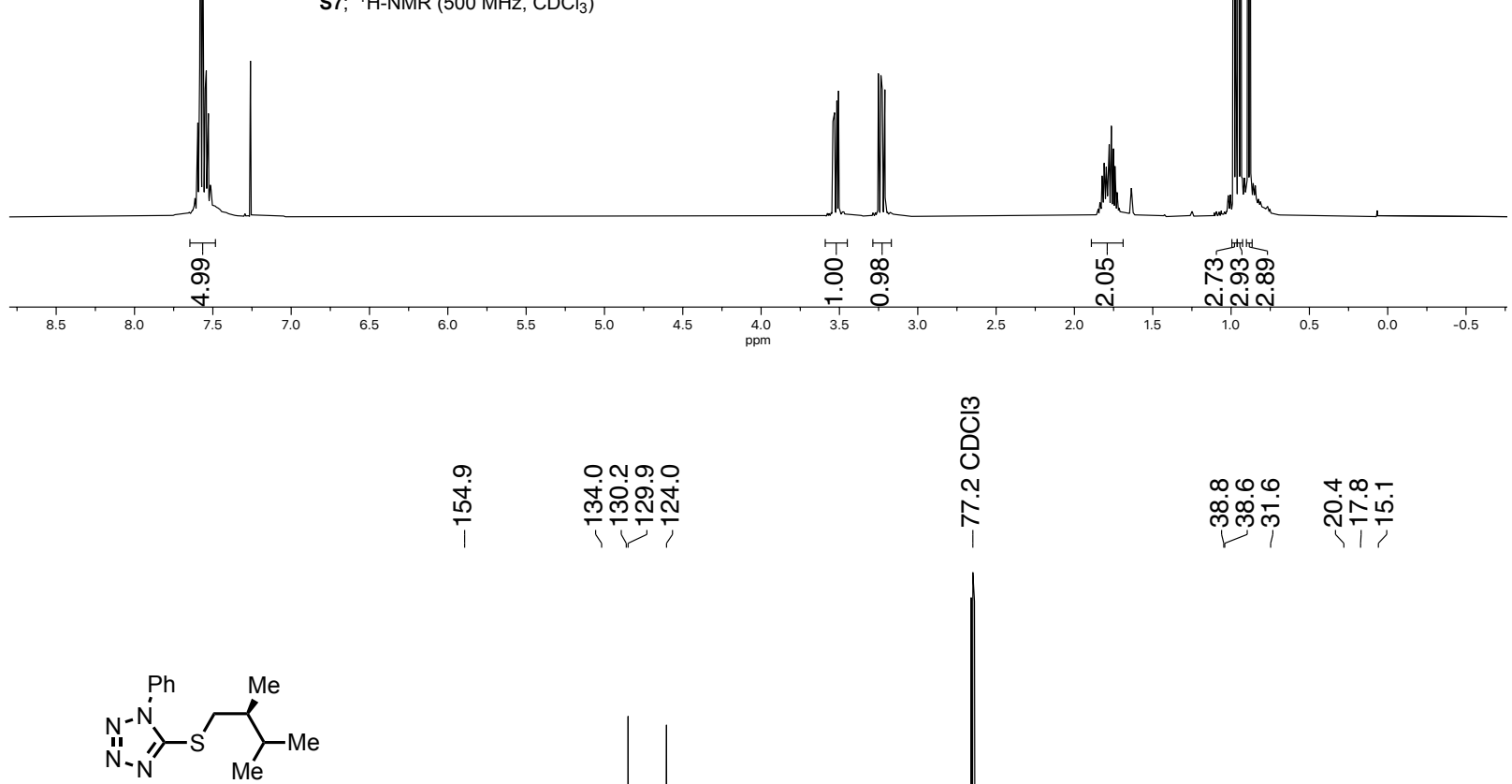

s7; ${ }^{13} \mathrm{C}-\mathrm{NMR}\left(126 \mathrm{MHz}, \mathrm{CDCl}_{3}\right.$ )
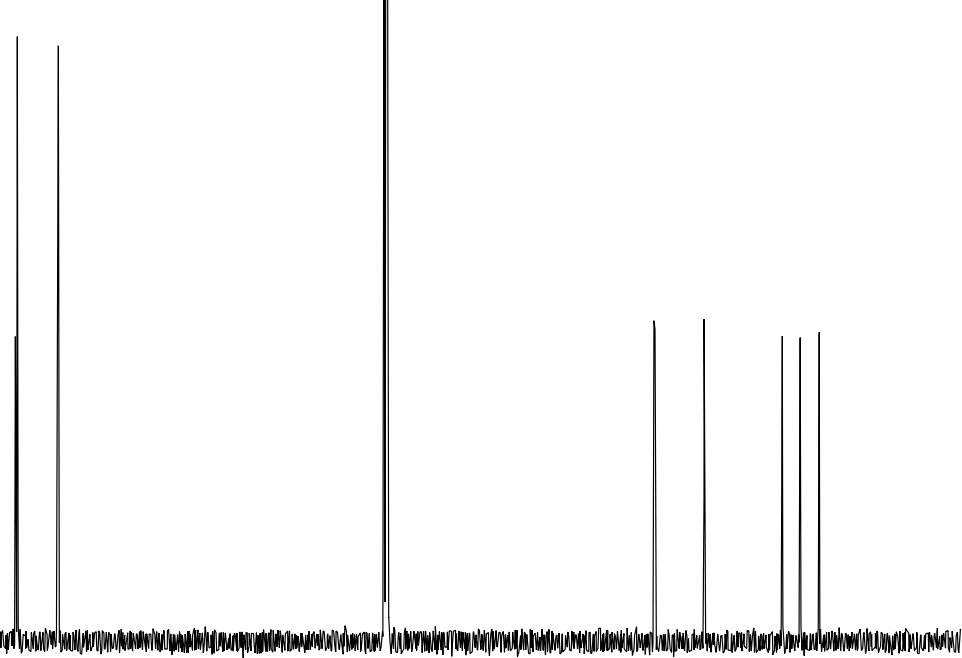

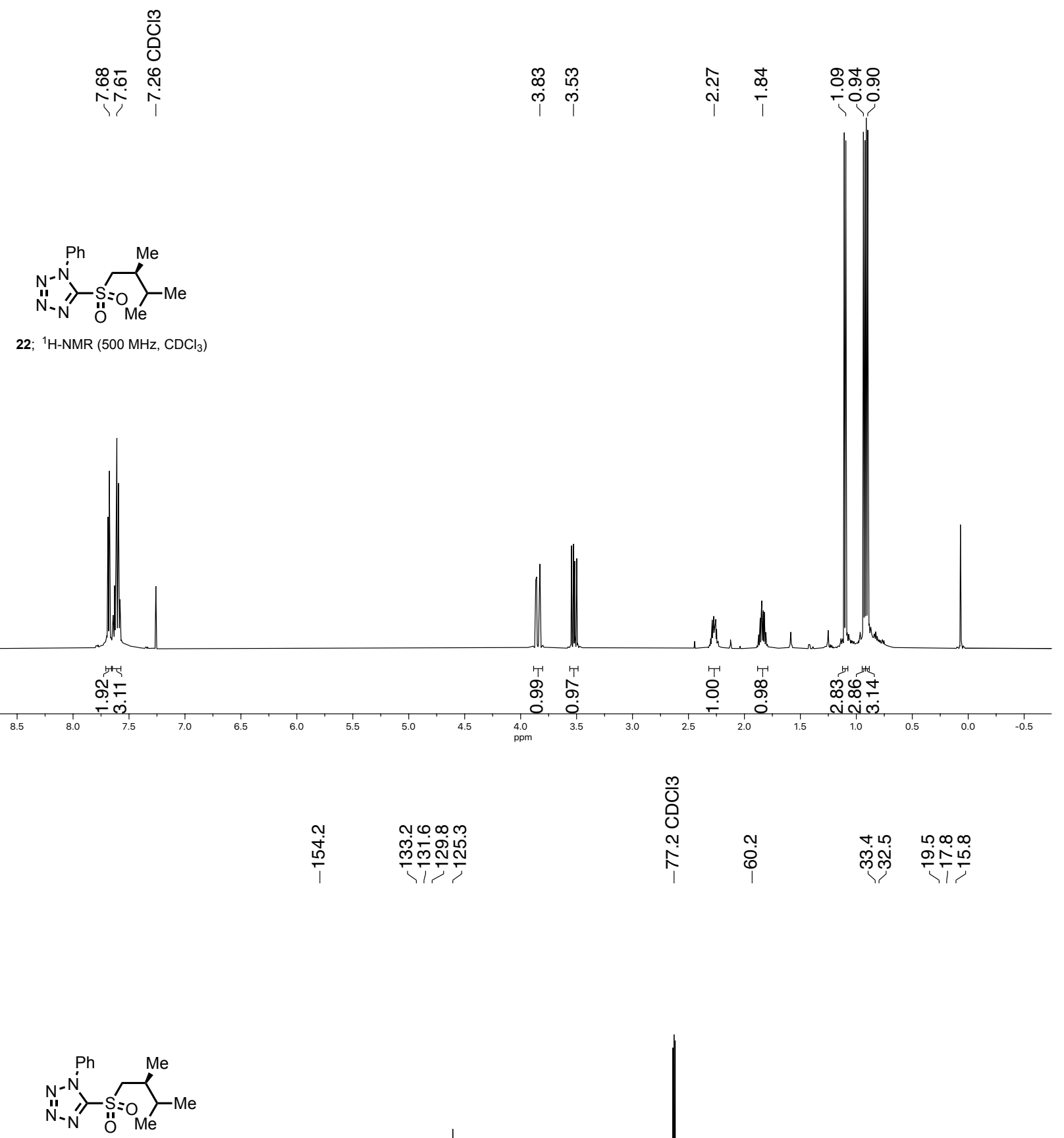

22; ${ }^{13} \mathrm{C}-\mathrm{NMR}\left(126 \mathrm{MHz}, \mathrm{CDCl}_{3}\right)$

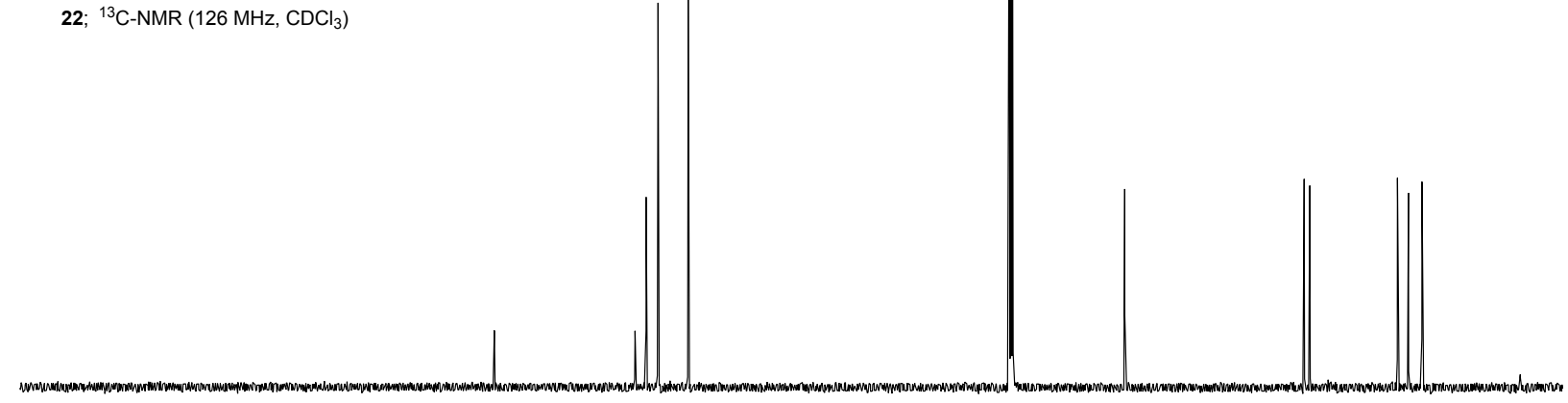

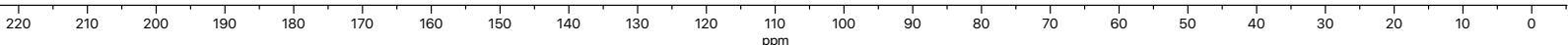




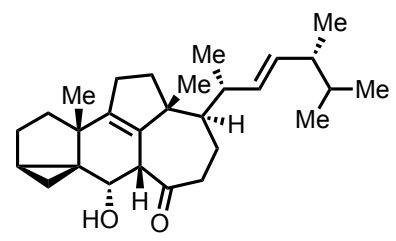

13; ${ }^{1} \mathrm{H}-\mathrm{NMR}\left(500 \mathrm{MHz}, \mathrm{CDCl}_{3}\right)$
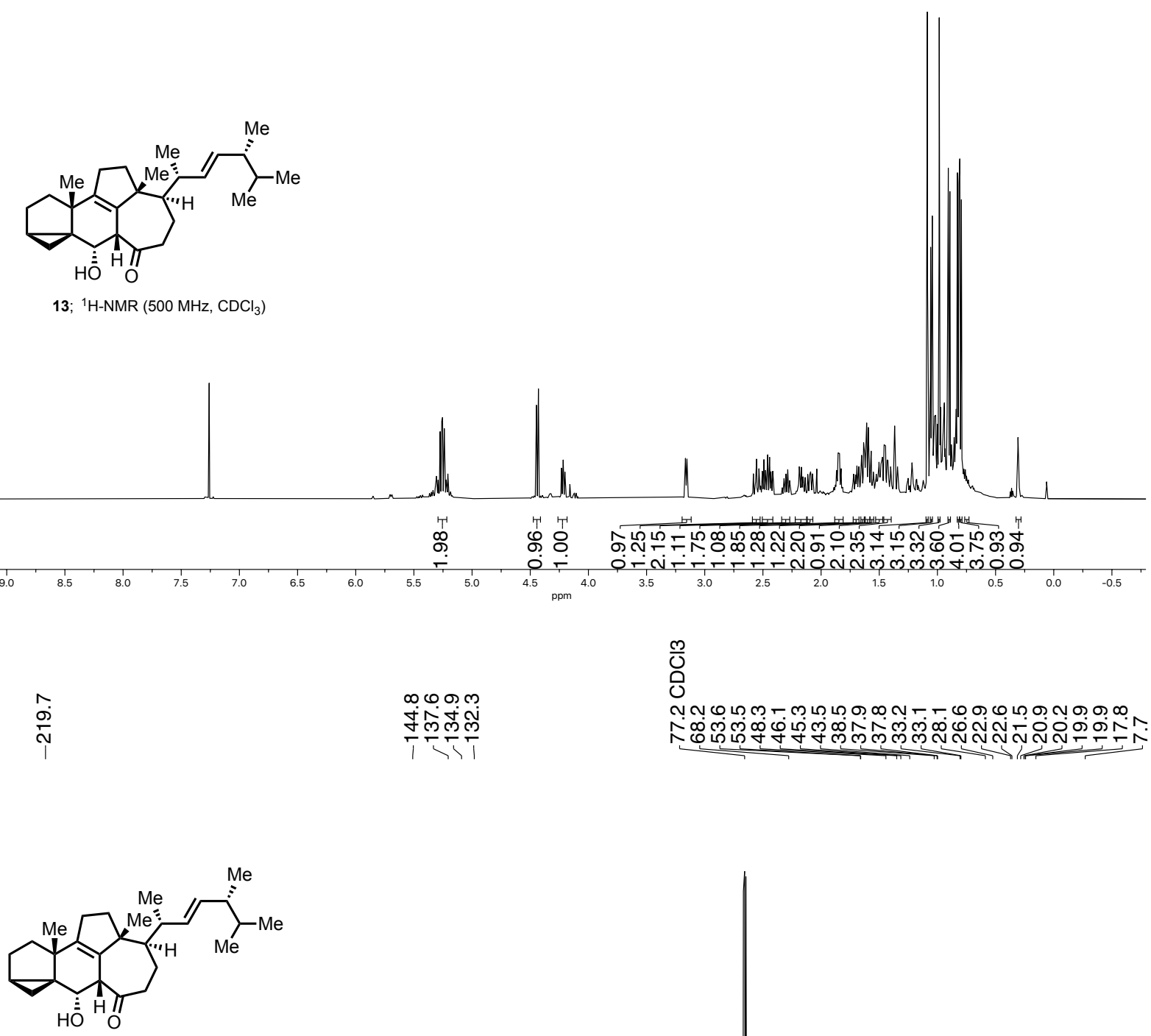

13; ${ }^{13} \mathrm{C}-\mathrm{NMR}\left(126 \mathrm{MHz}, \mathrm{CDCl}_{3}\right)$

$\frac{m}{0}$

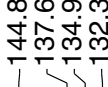

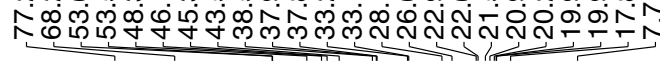
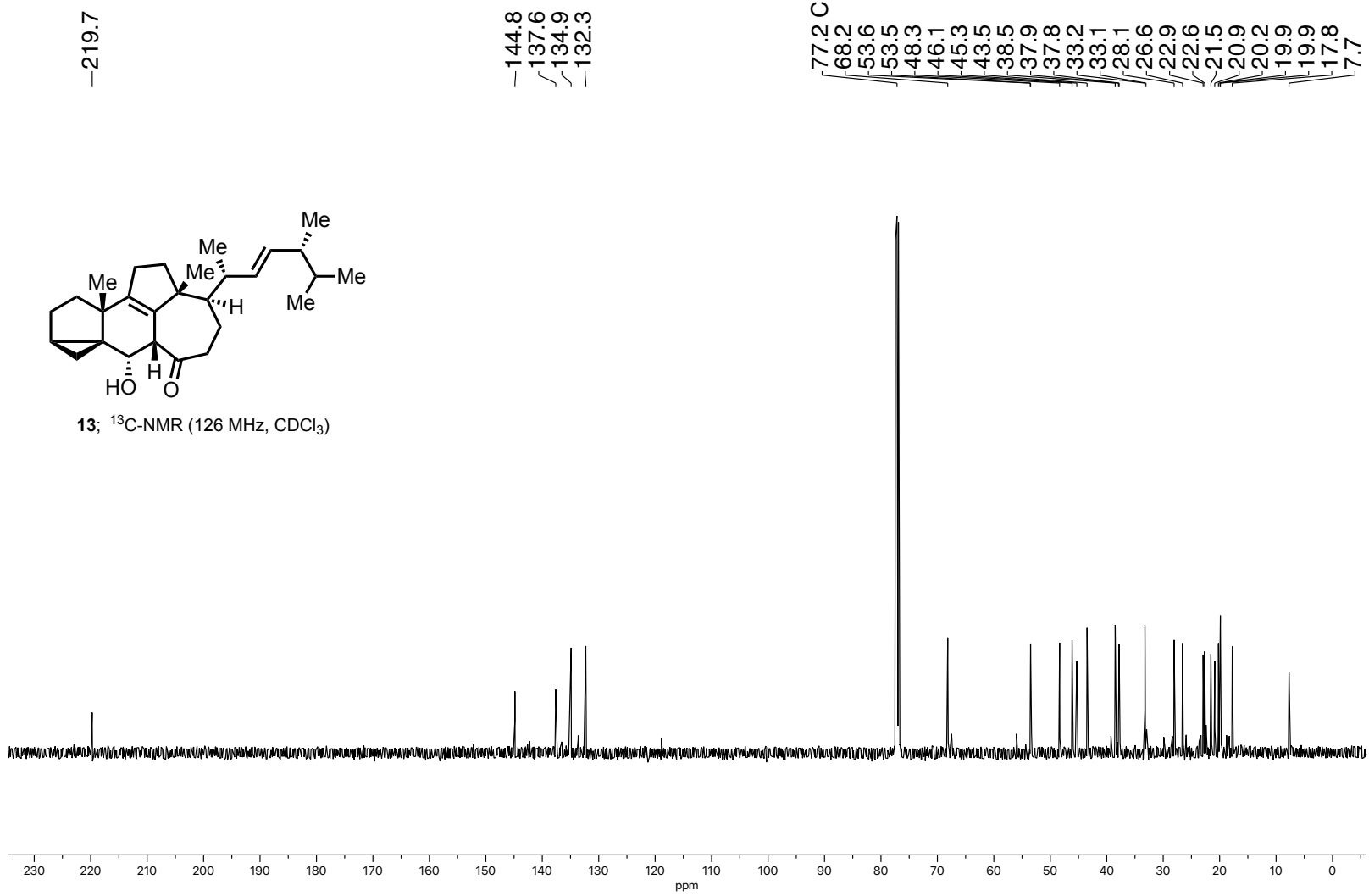

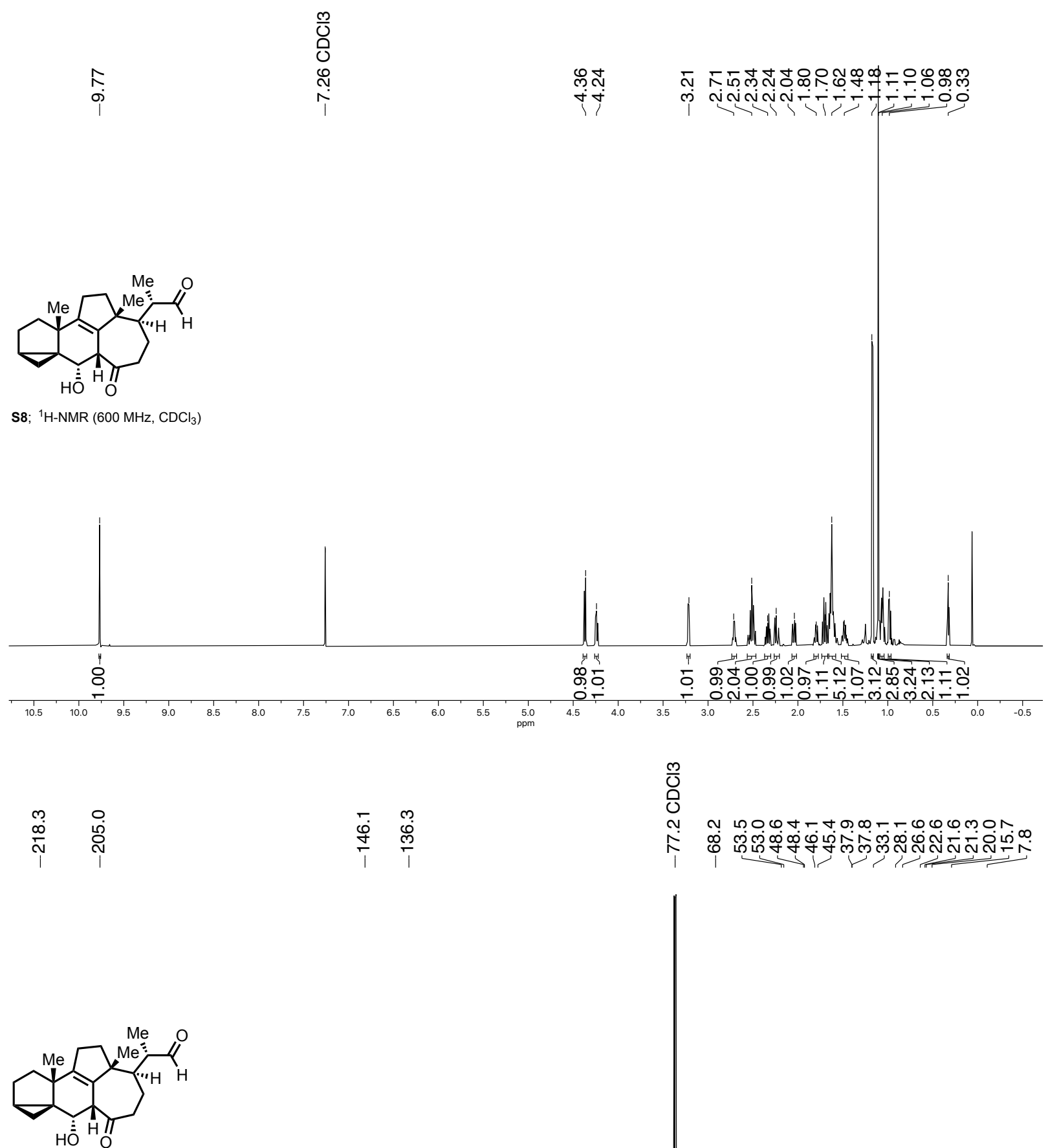

S8; ${ }^{13} \mathrm{C}-\mathrm{NMR}\left(151 \mathrm{MHz}, \mathrm{CDCl}_{3}\right)$
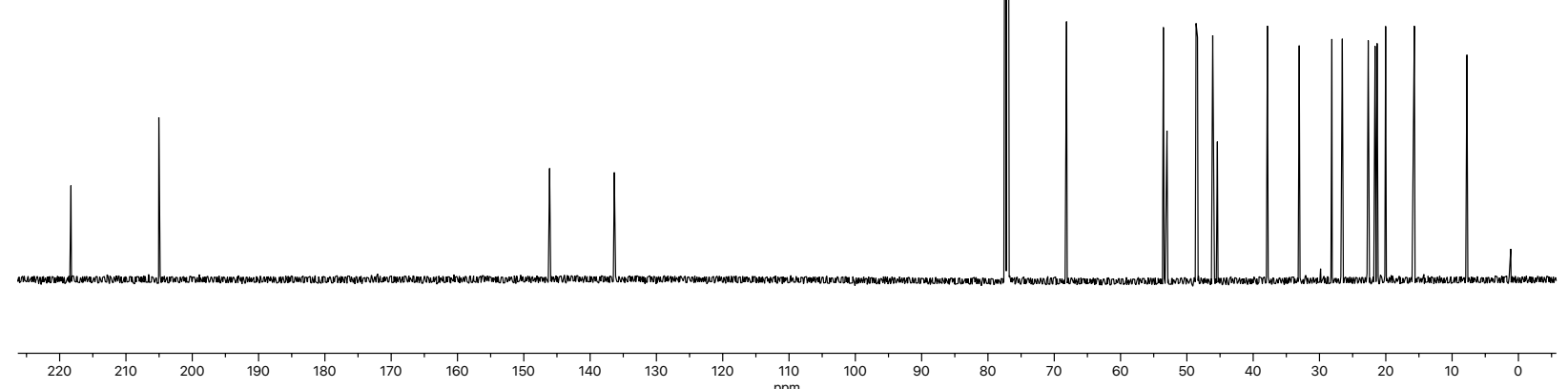

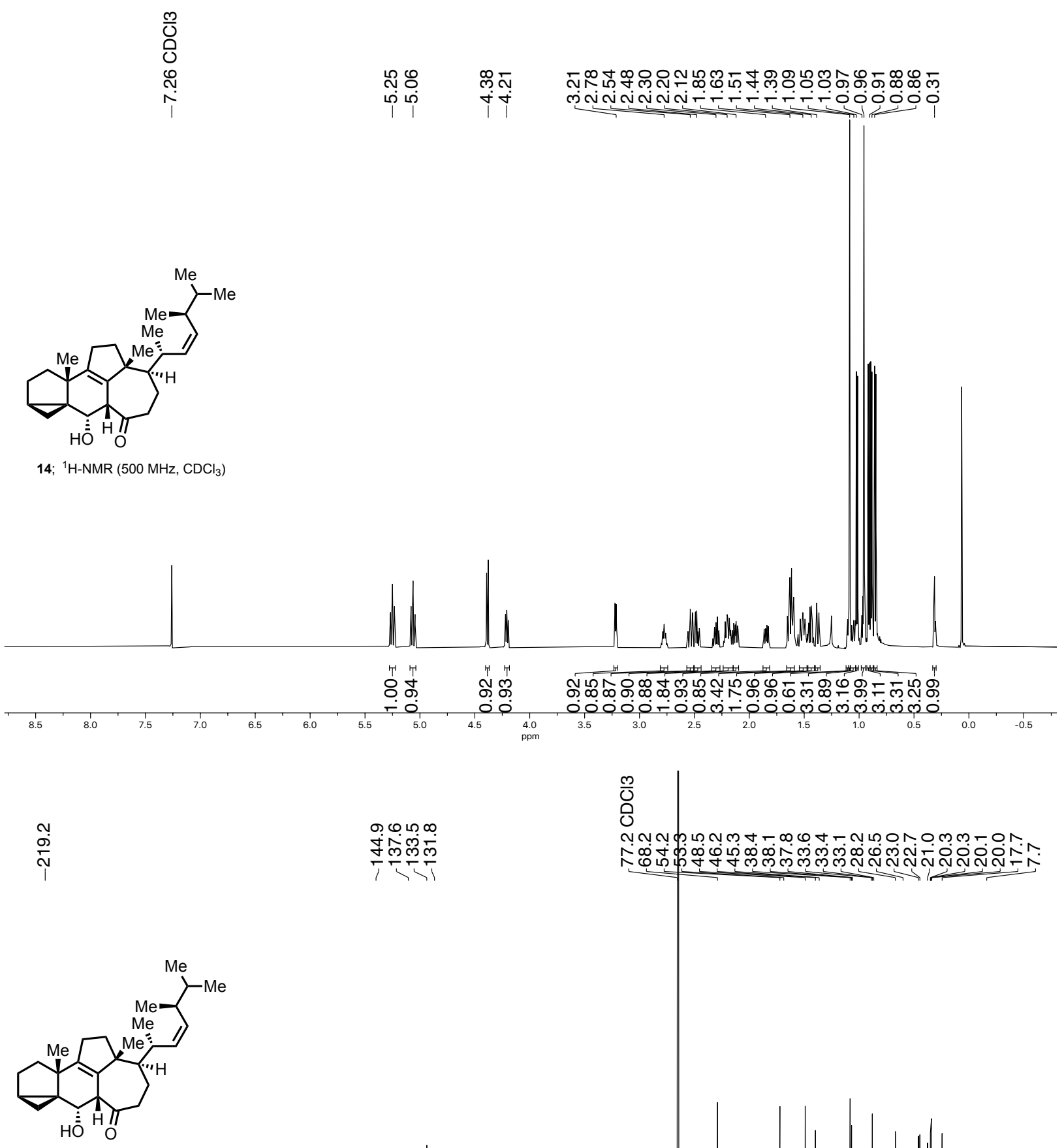

14; ${ }^{13} \mathrm{C}-\mathrm{NMR}\left(126 \mathrm{MHz}, \mathrm{CDCl}_{3}\right)$
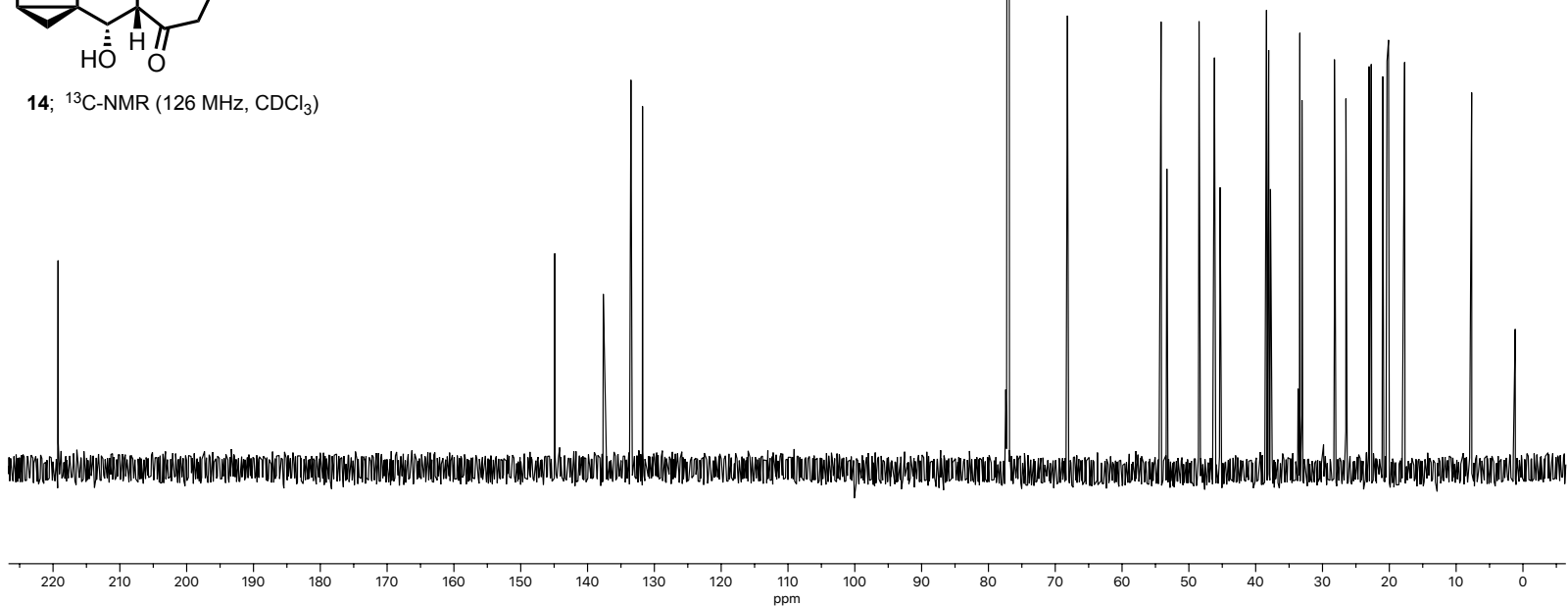

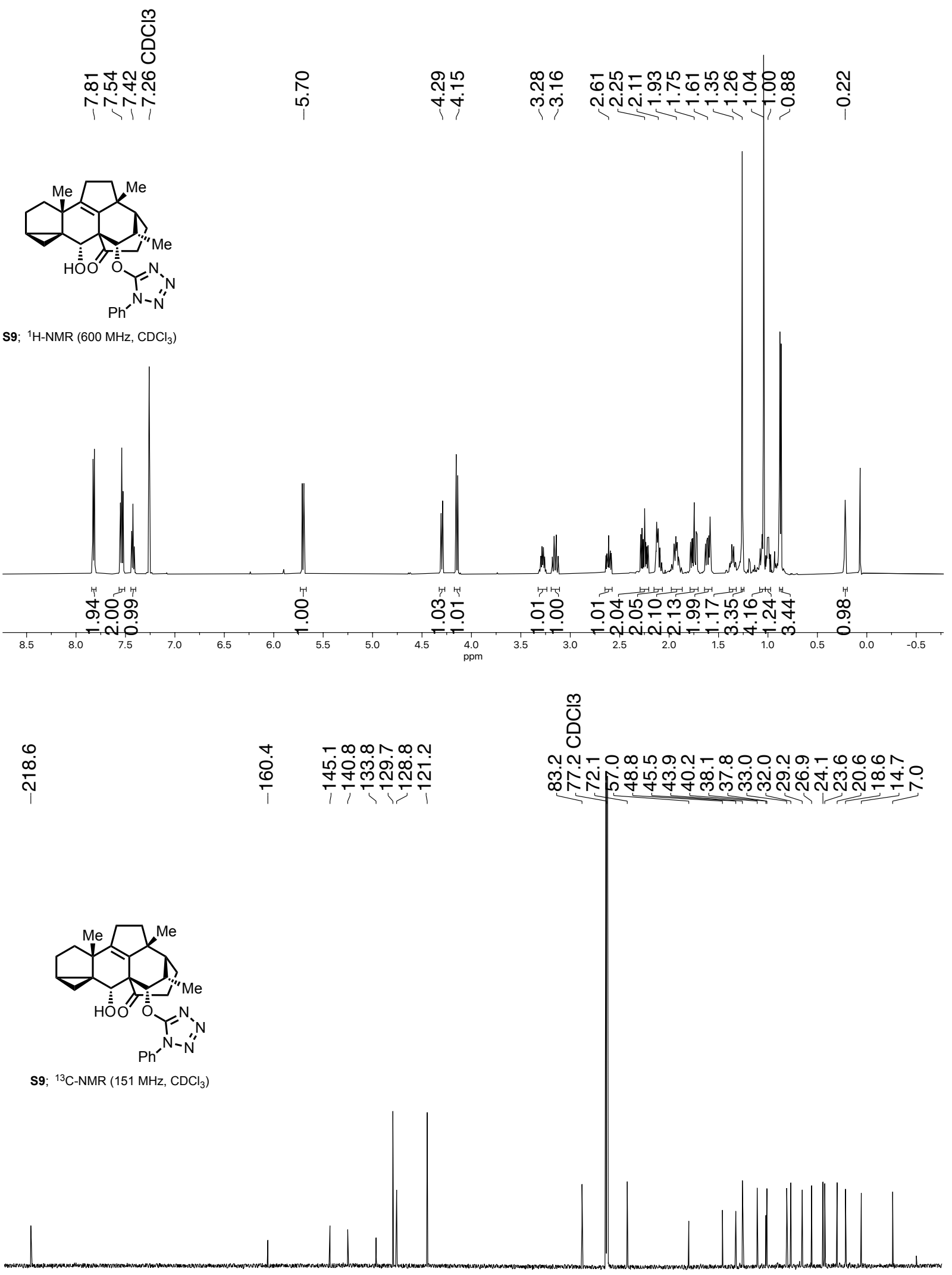

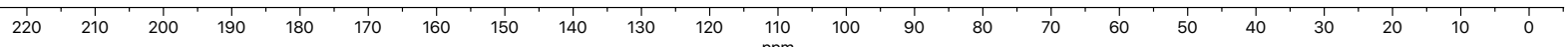



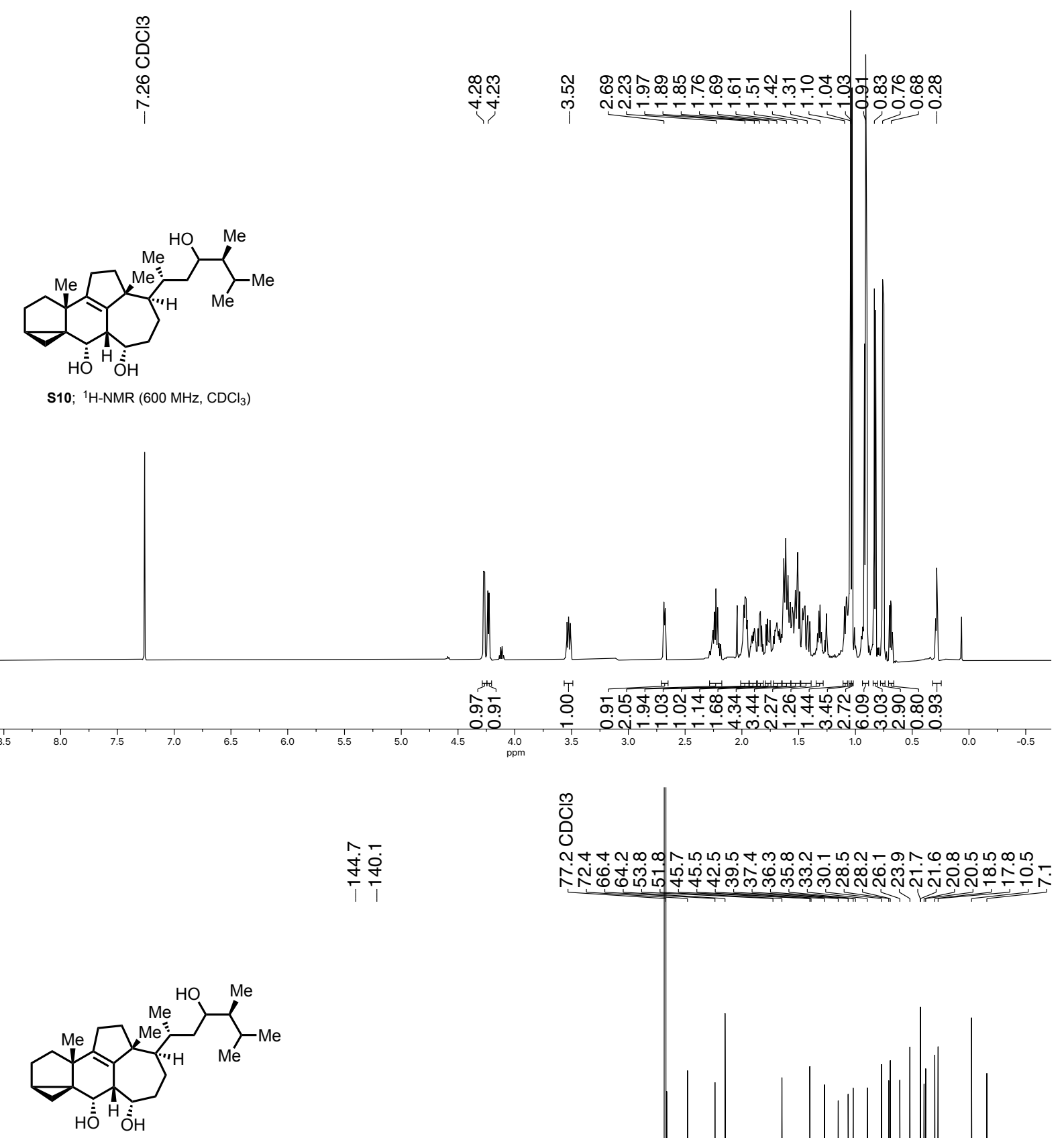

S10; ${ }^{13} \mathrm{C}-\mathrm{NMR}\left(151 \mathrm{MHz}, \mathrm{CDCl}_{3}\right)$

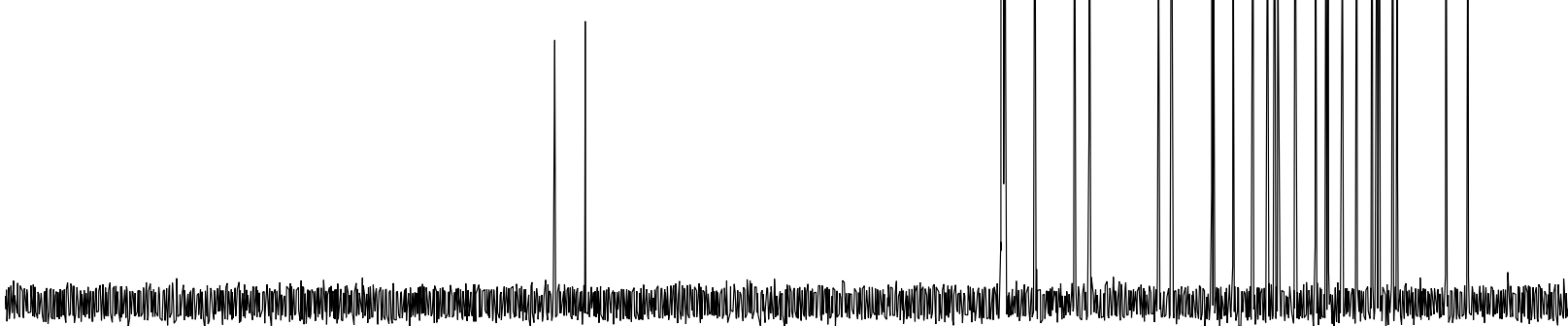

$\begin{array}{llllllllllll}220 & 210 & 200 & 190 & 180 & 170 & 160 & 150 & 140 & 130 & 120 & 110\end{array}$ 


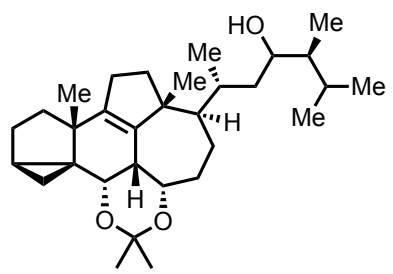

S11a; ${ }^{1} \mathrm{H}-\mathrm{NMR}\left(500 \mathrm{MHz}, \mathrm{CDCl}_{3}\right)$
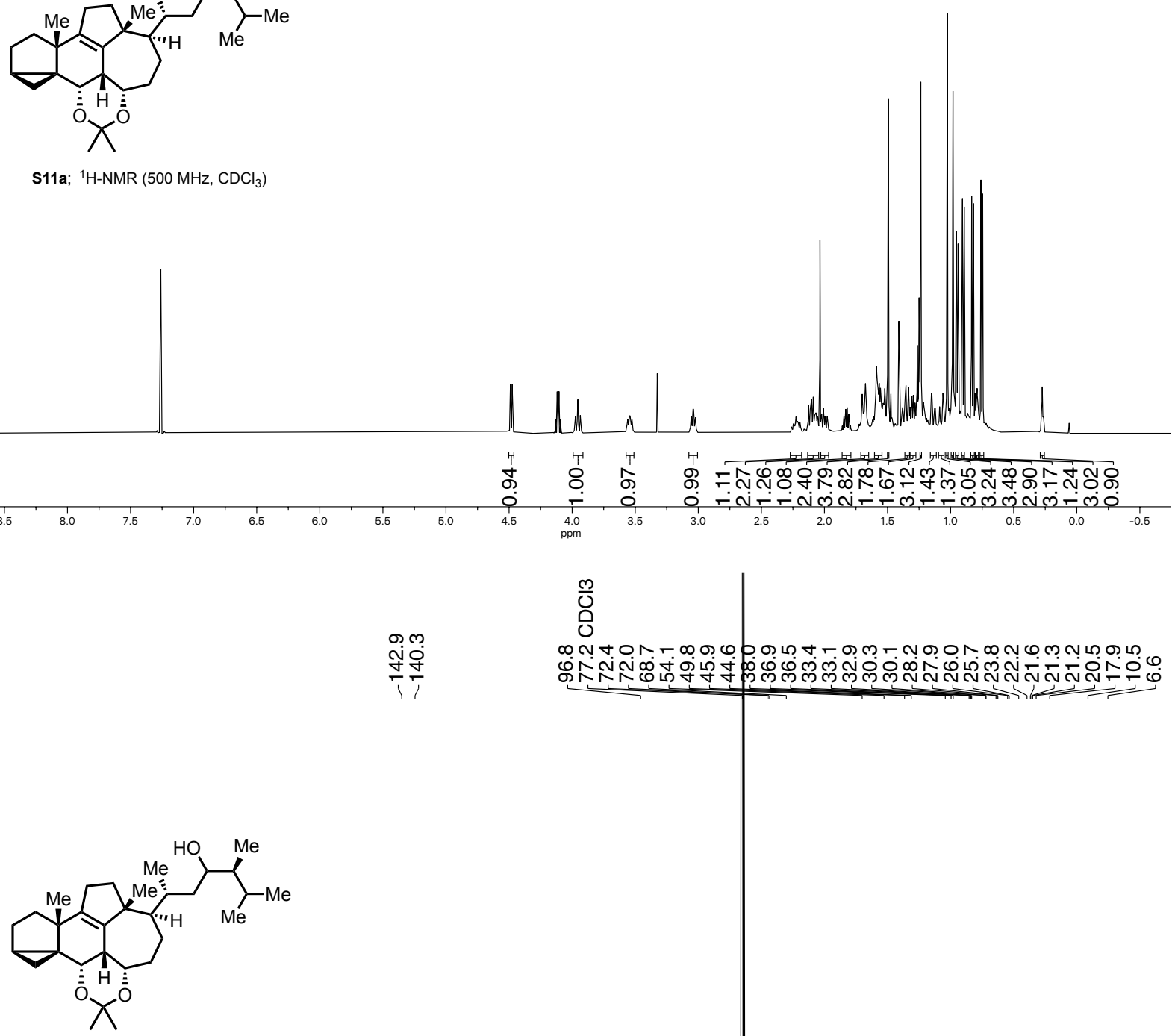

S11a; ${ }^{13} \mathrm{C}-\mathrm{NMR}\left(126 \mathrm{MHz}, \mathrm{CDCl}_{3}\right)$

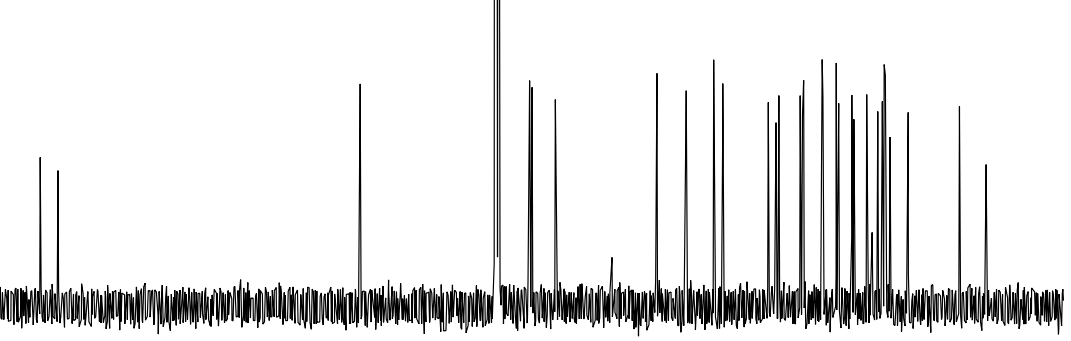

$\begin{array}{lllllllllll}210 & 200 & 190 & 180 & 170 & 160 & 150 & 140 & 130 & 120 & 110\end{array}$ 

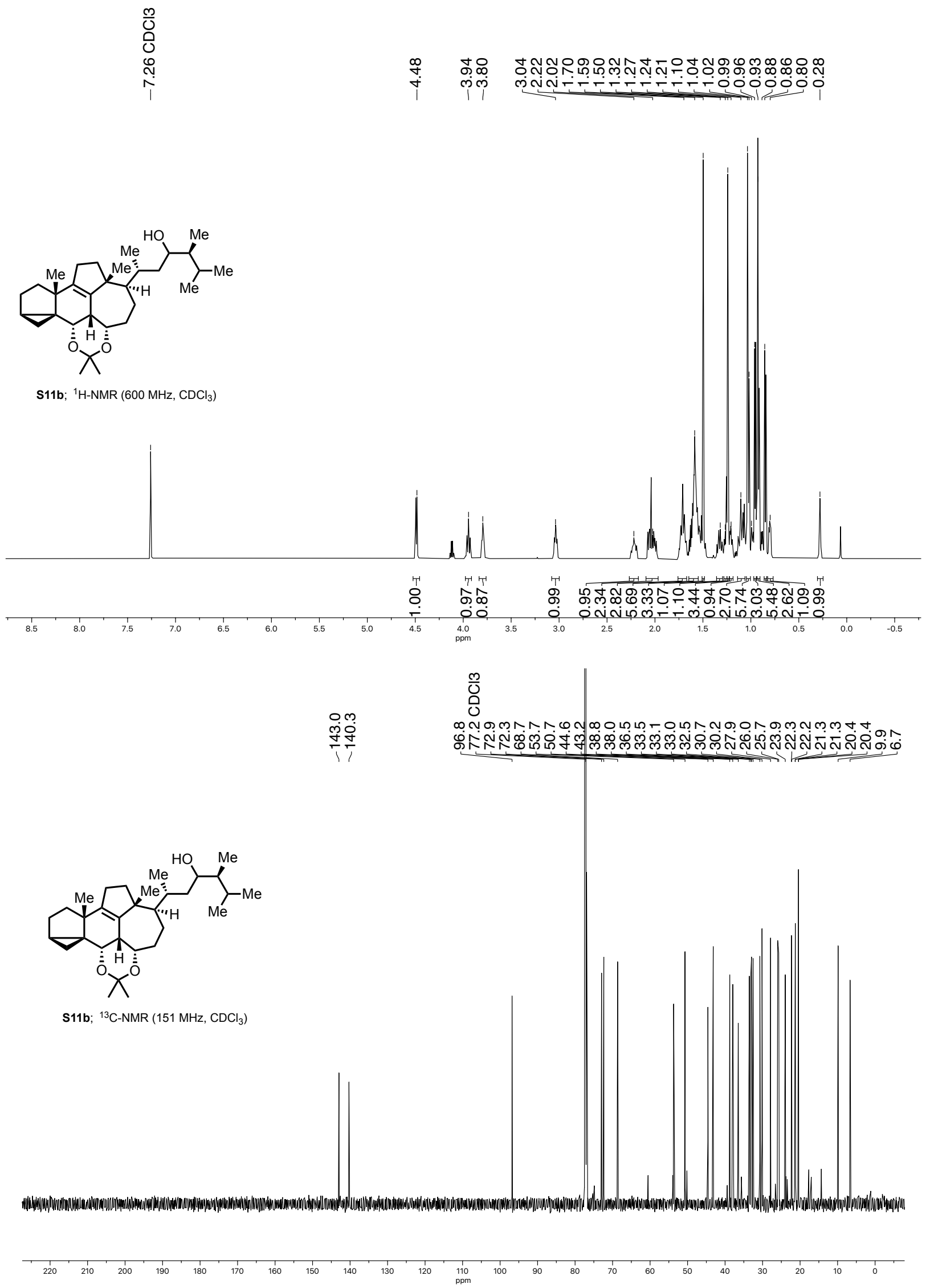

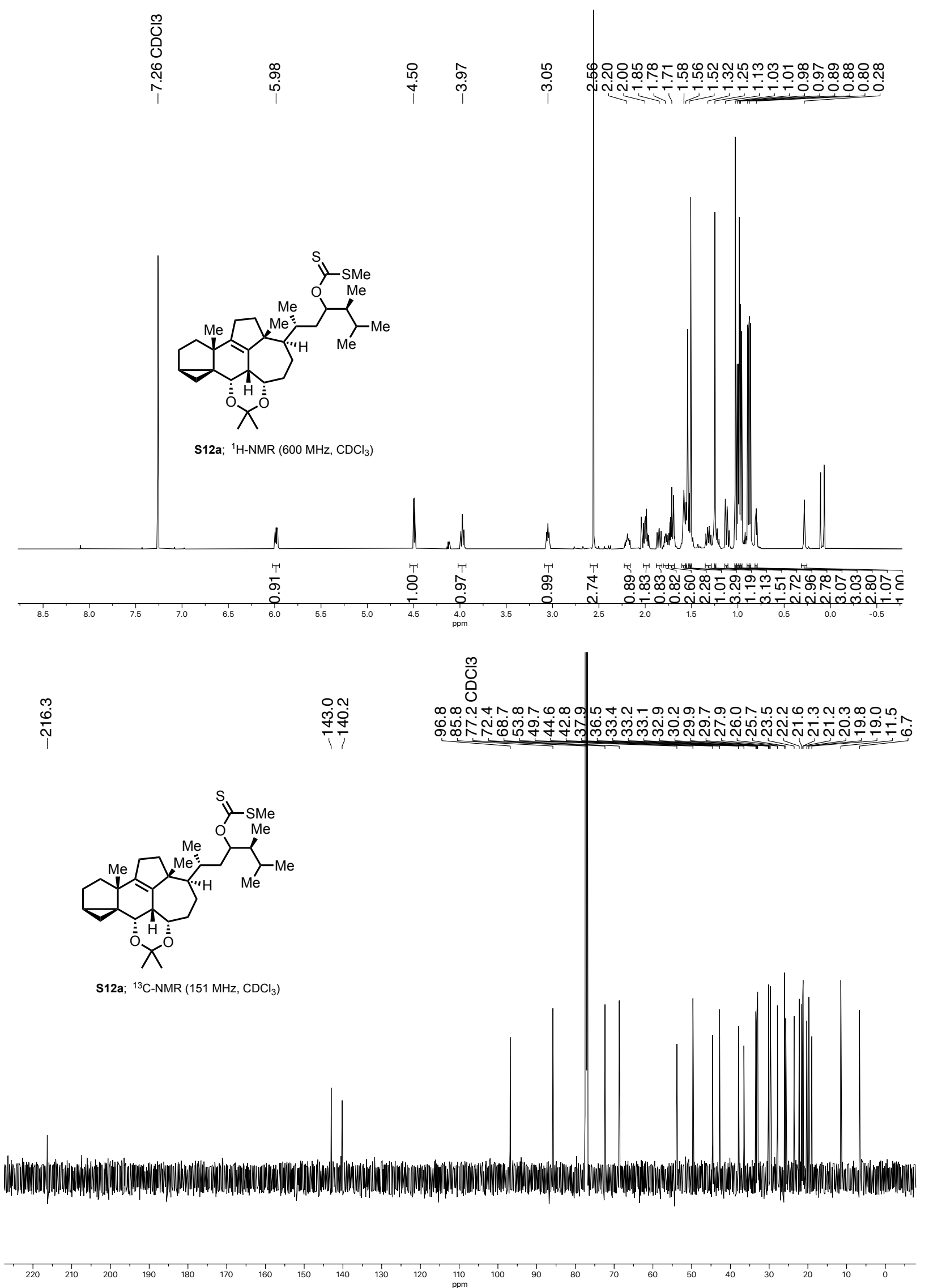

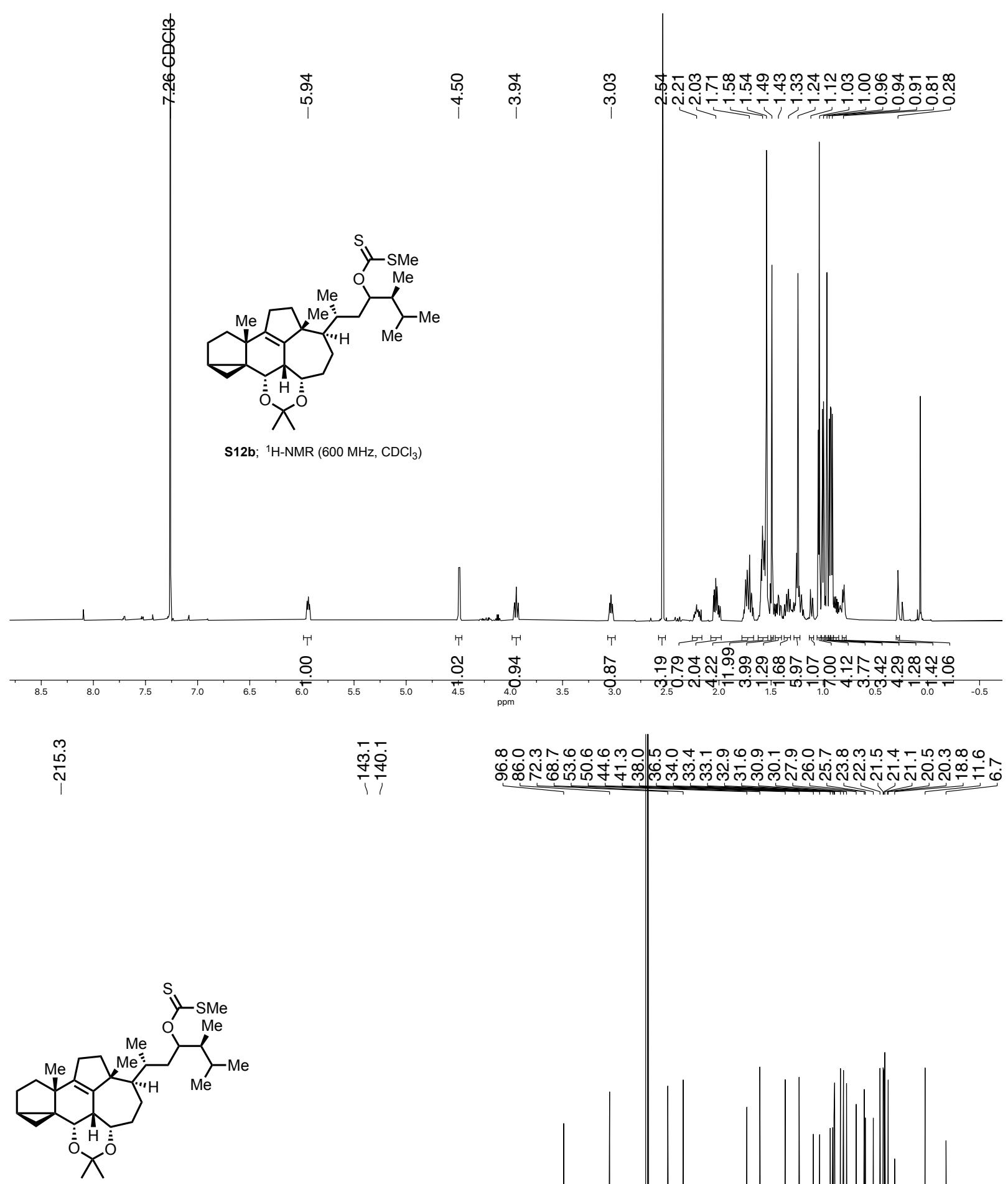

S12b; ${ }^{13} \mathrm{C}-\mathrm{NMR}\left(151 \mathrm{MHz}, \mathrm{CDCl}_{3}\right)$

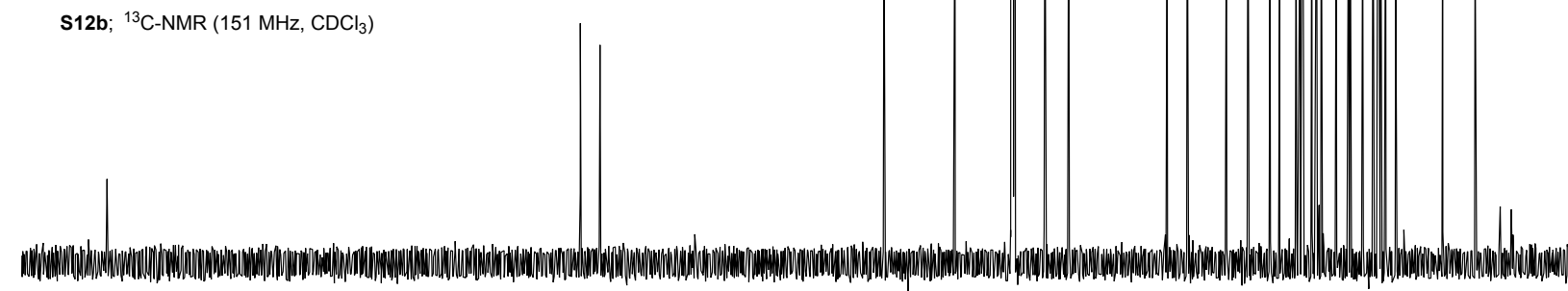

$\begin{array}{llllllllllll}220 & 210 & 200 & 190 & 180 & 170 & 160 & 150 & 140 & 130 & 120 & \begin{array}{r}1 \\ 110\end{array} \\ p p p\end{array}$ 
$\frac{m}{0}$
0
0
0
$\stackrel{N}{1}$
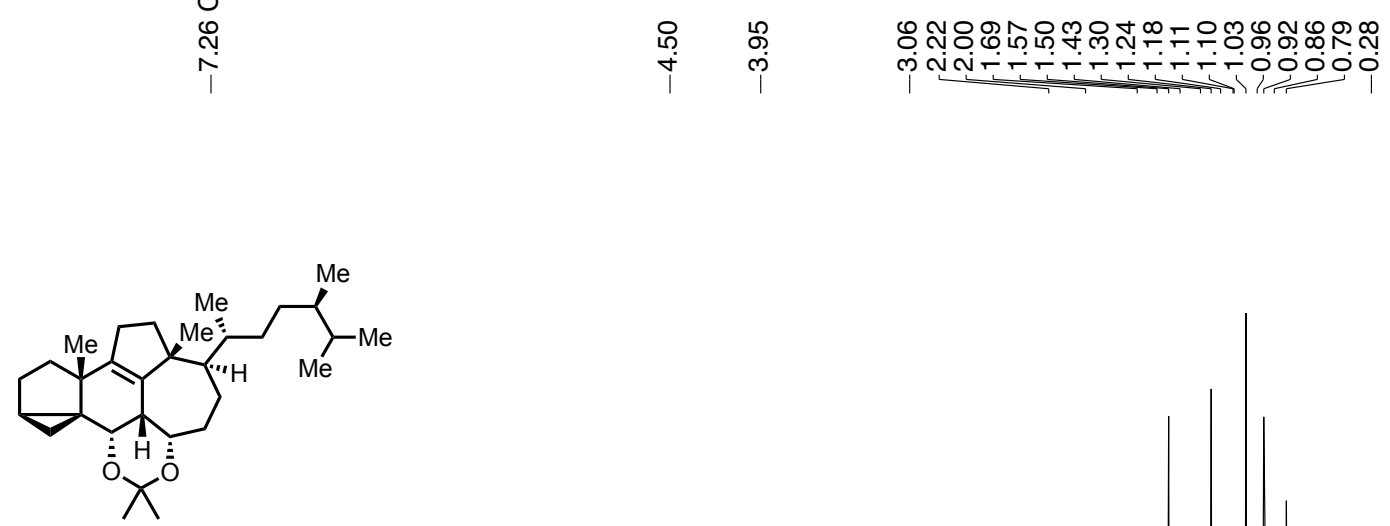

15; ${ }^{1} \mathrm{H}-\mathrm{NMR}\left(400 \mathrm{MHz}, \mathrm{CDCl}_{3}\right)$
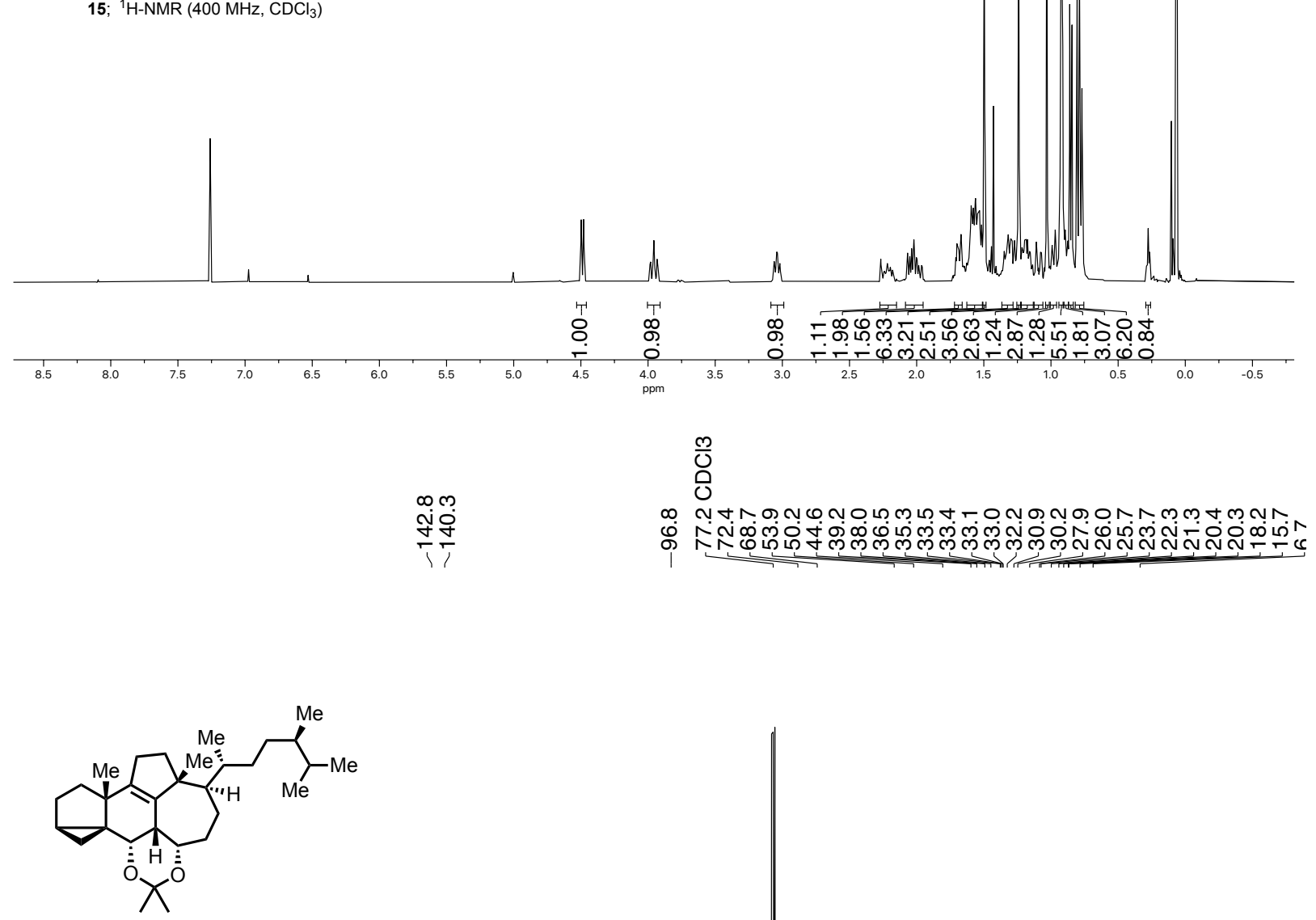

15; ${ }^{13} \mathrm{C}-\mathrm{NMR}\left(101 \mathrm{MHz}, \mathrm{CDCl}_{3}\right)$
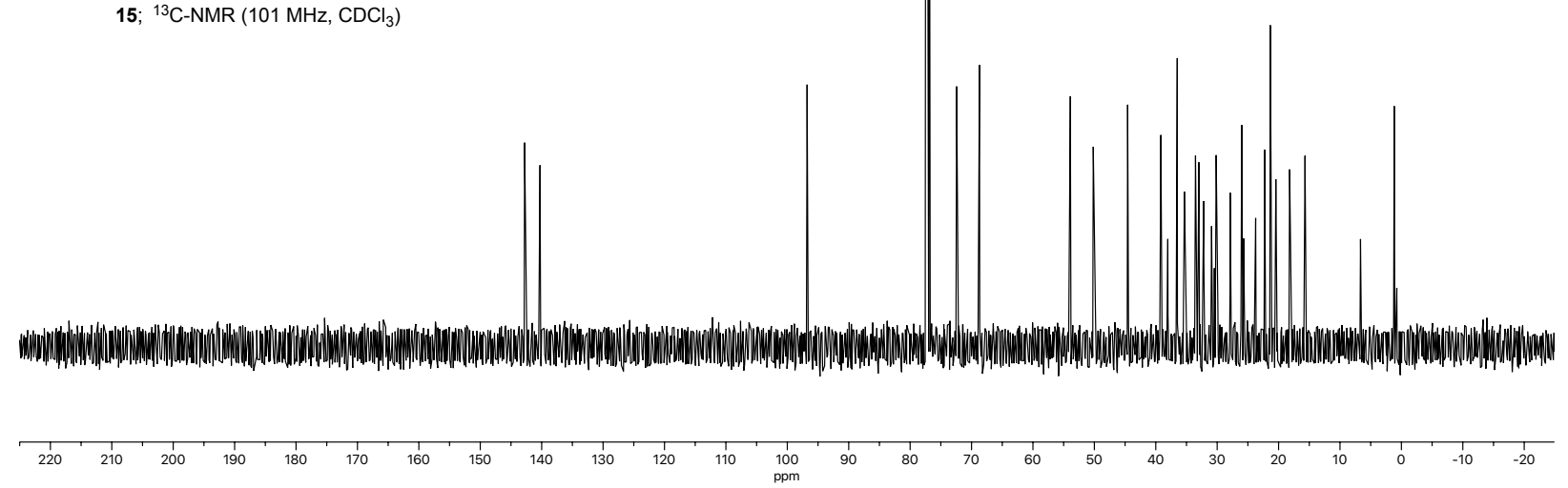

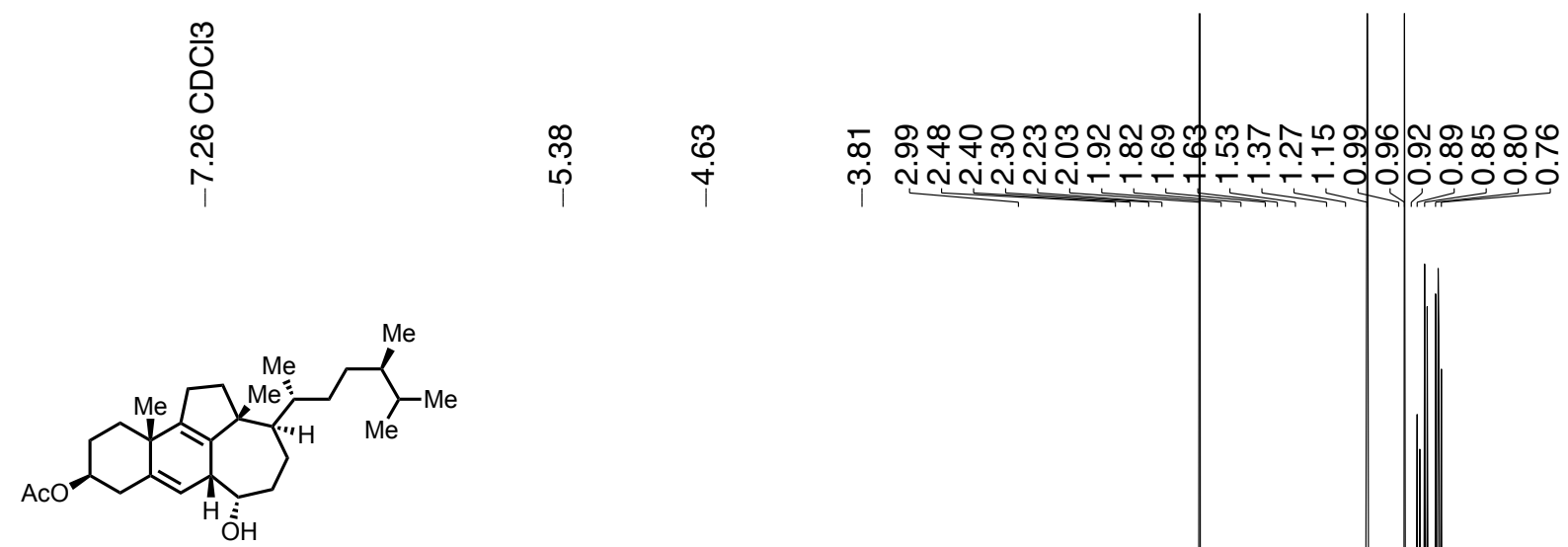

S13; ${ }^{1} \mathrm{H}-\mathrm{NMR}\left(500 \mathrm{MHz}, \mathrm{CDCl}_{3}\right)$
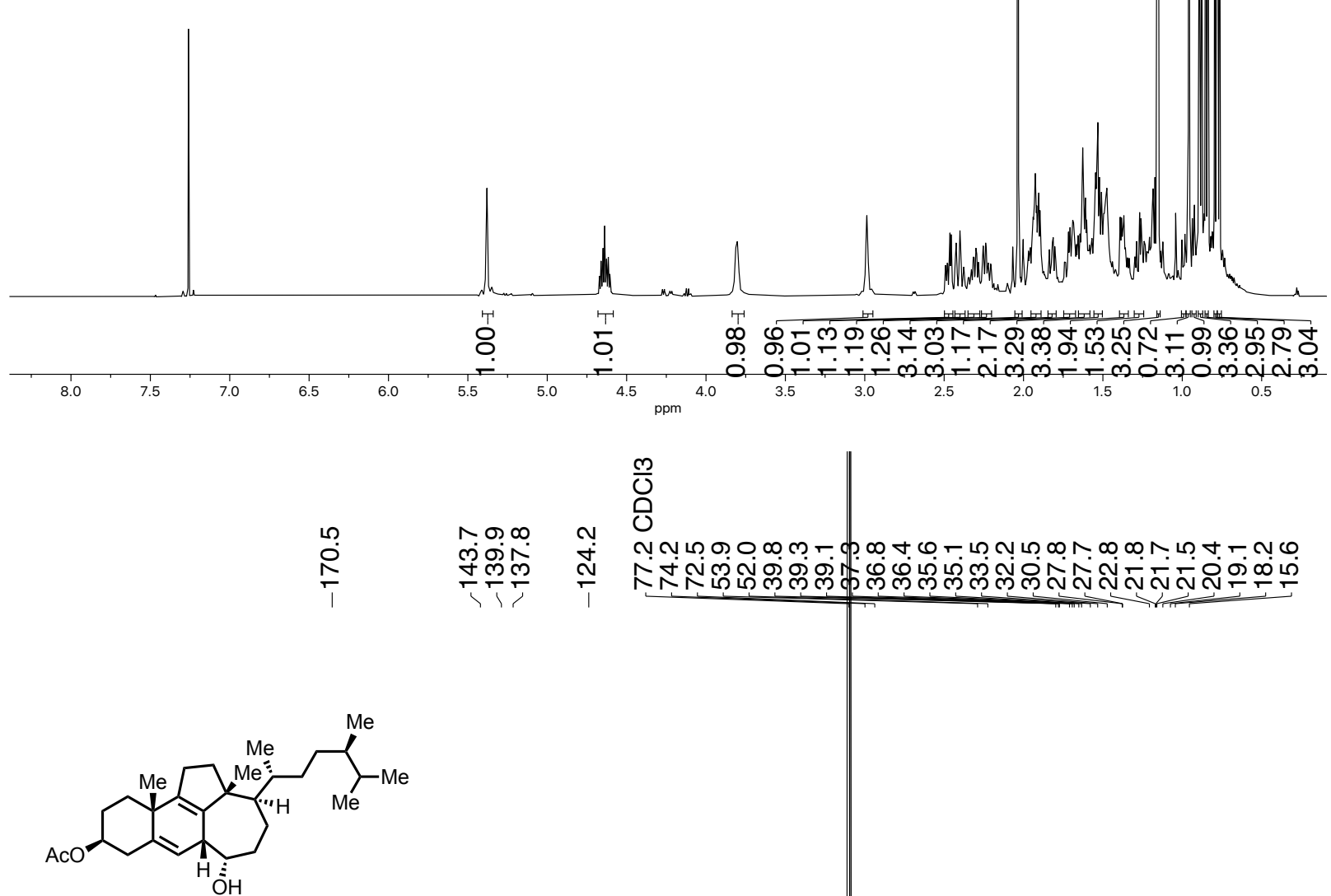

S13; ${ }^{13} \mathrm{C}-\mathrm{NMR}\left(126 \mathrm{MHz}, \mathrm{CDCl}_{3}\right)$
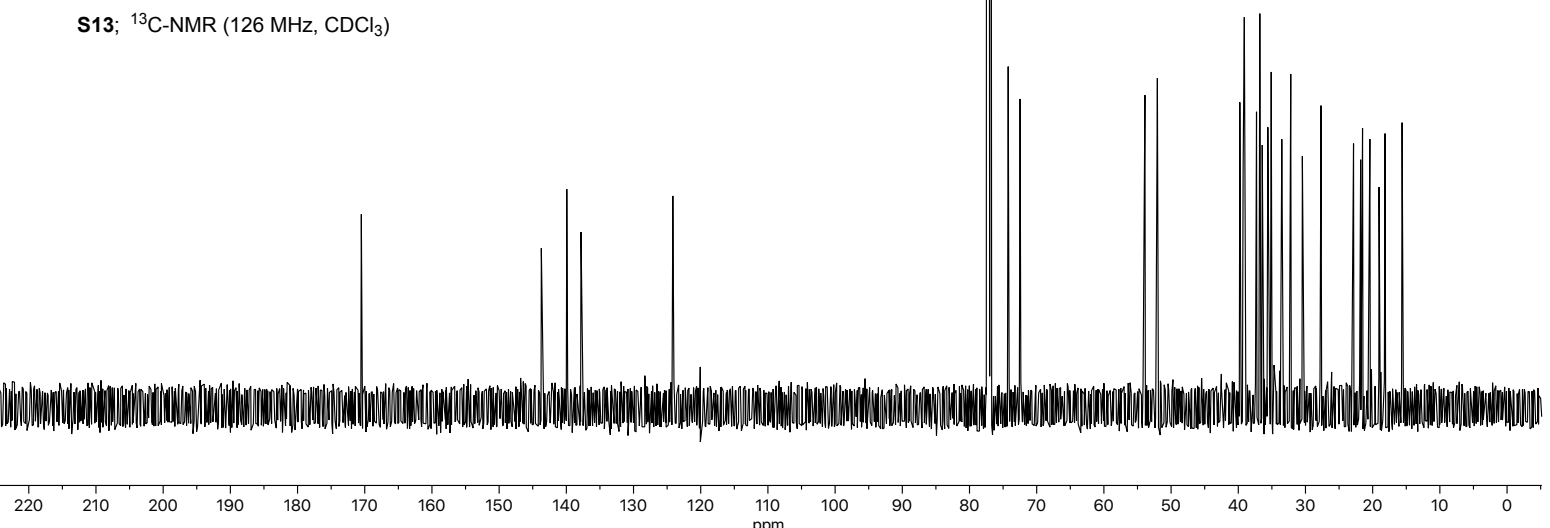

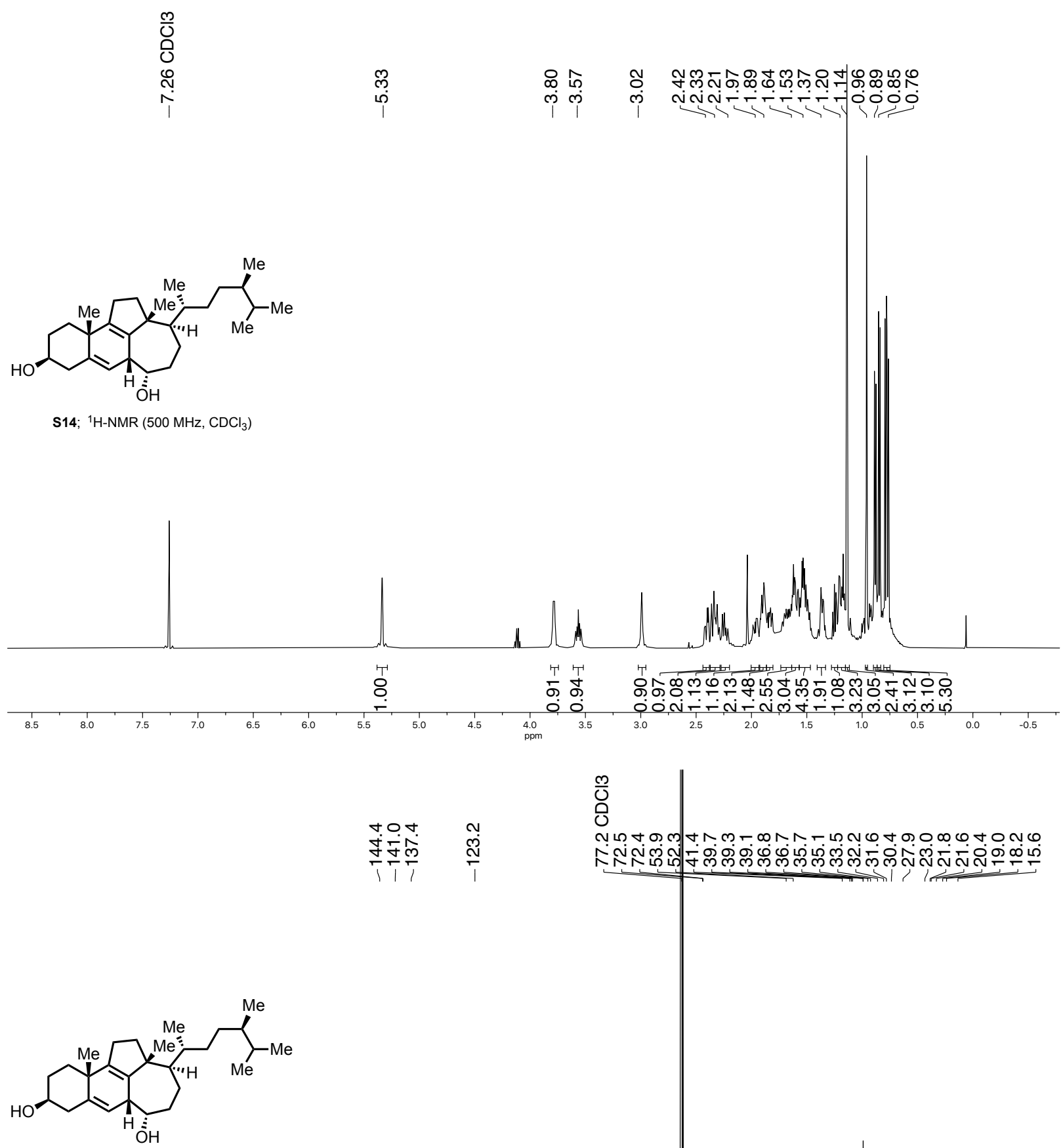

S14; ${ }^{13} \mathrm{C}-\mathrm{NMR}\left(126 \mathrm{MHz}, \mathrm{CDCl}_{3}\right)$

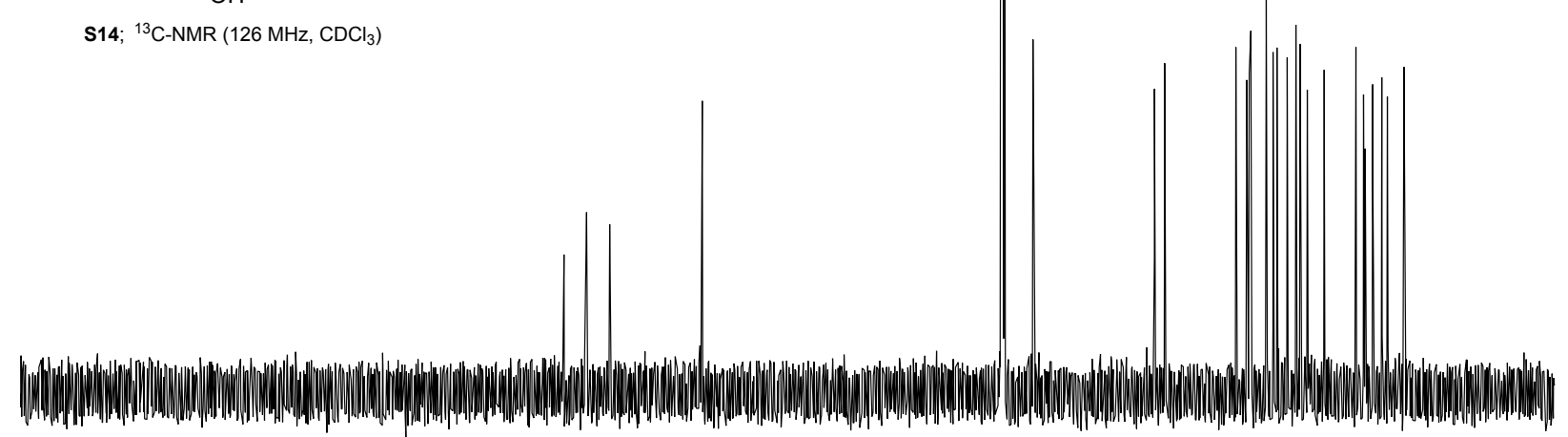

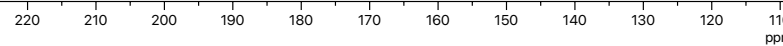



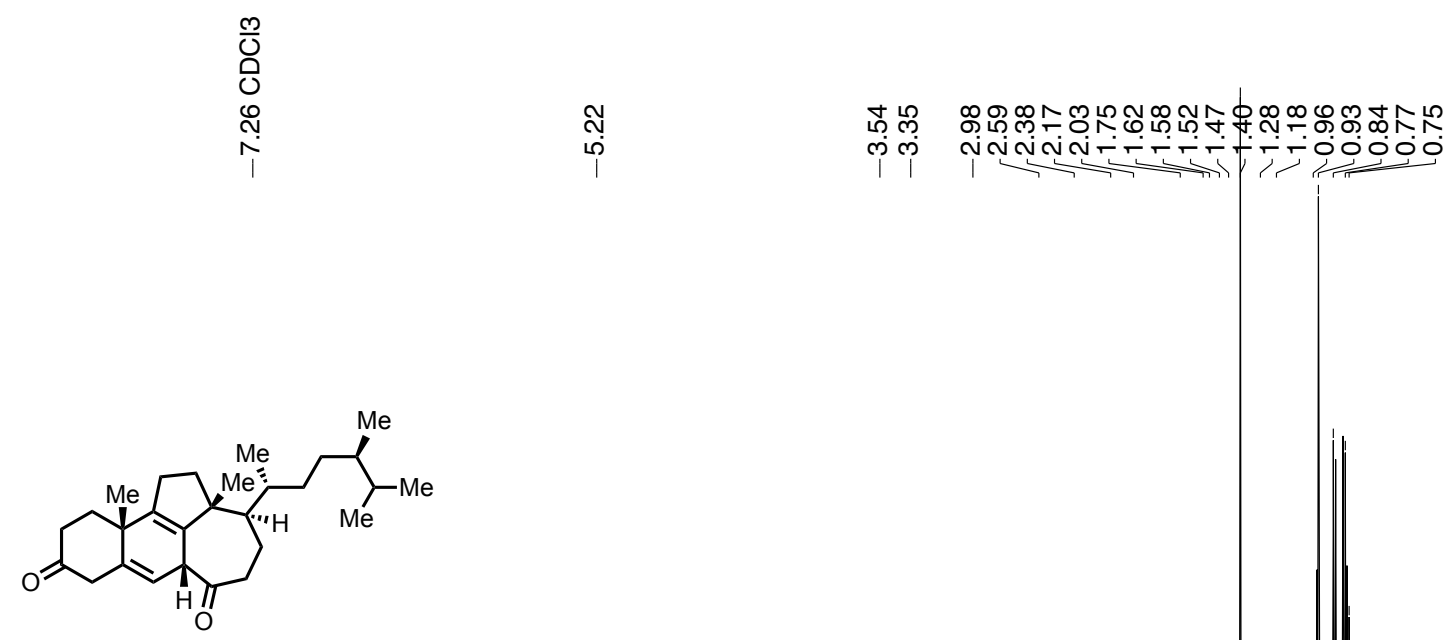

S15; ${ }^{13} \mathrm{C}-\mathrm{NMR}\left(126 \mathrm{MHz}, \mathrm{CDCl}_{3}\right)$

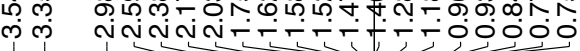
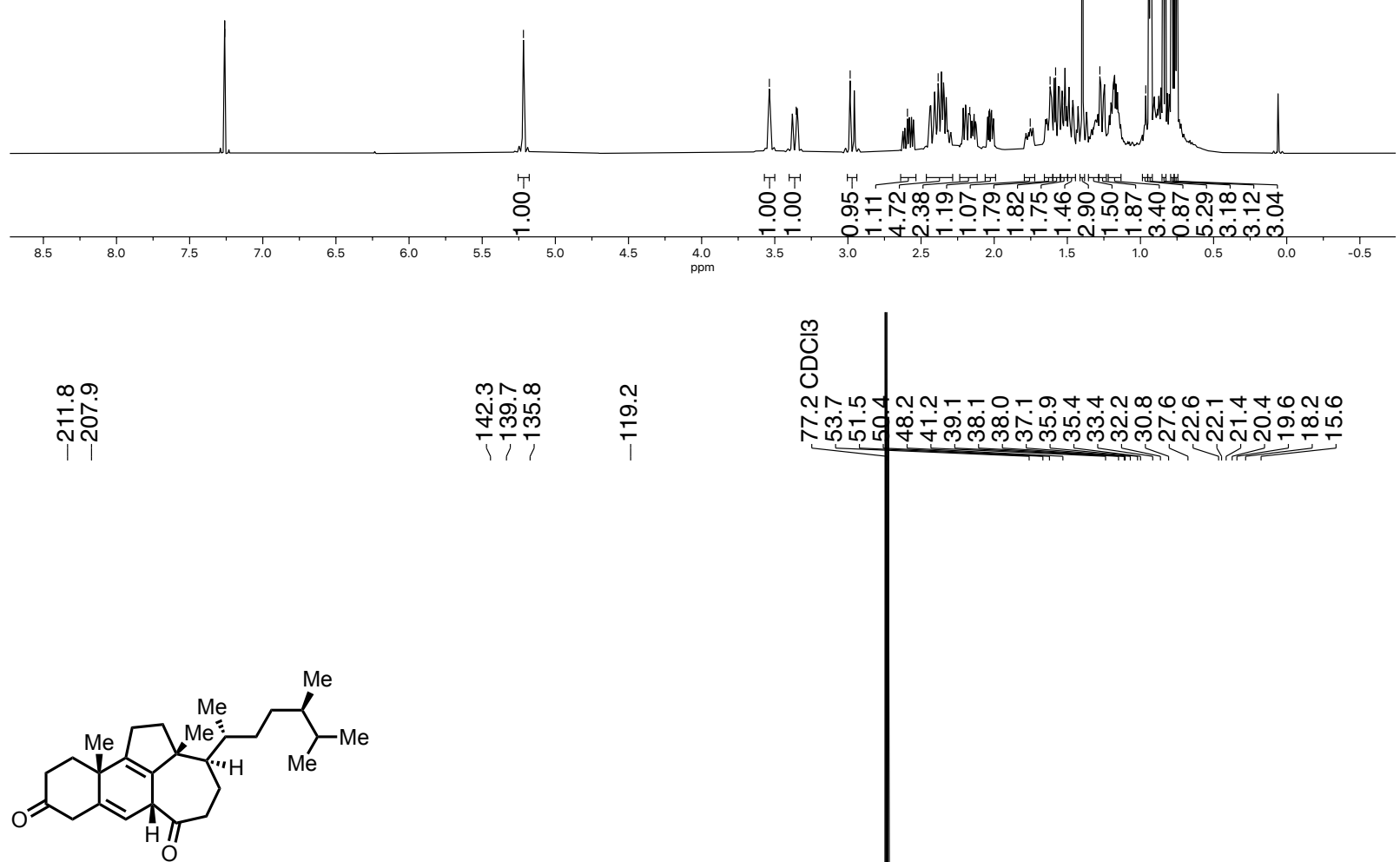

S15; ${ }^{1} \mathrm{H}-\mathrm{NMR}\left(500 \mathrm{MHz}, \mathrm{CDCl}_{3}\right)$
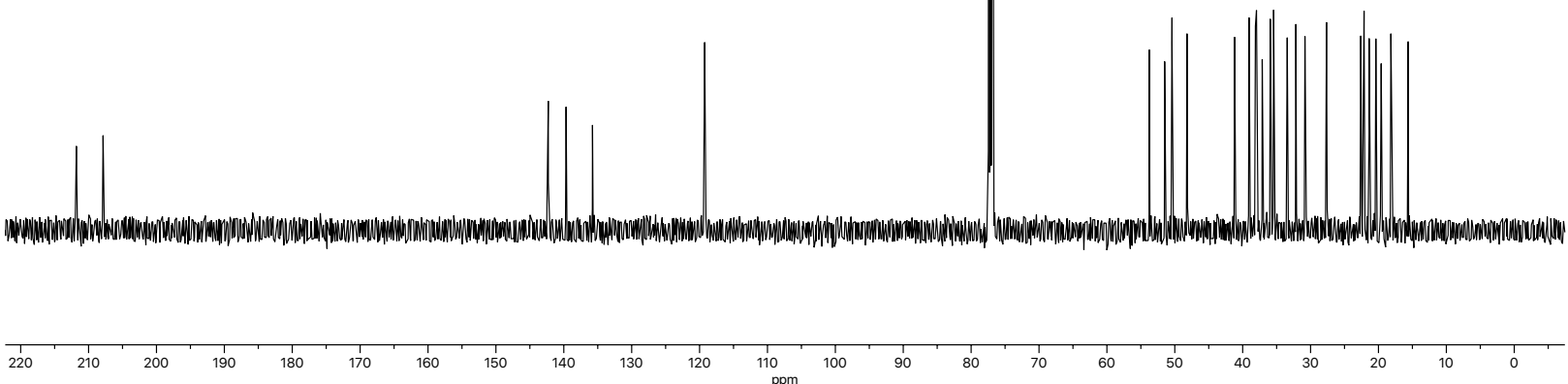

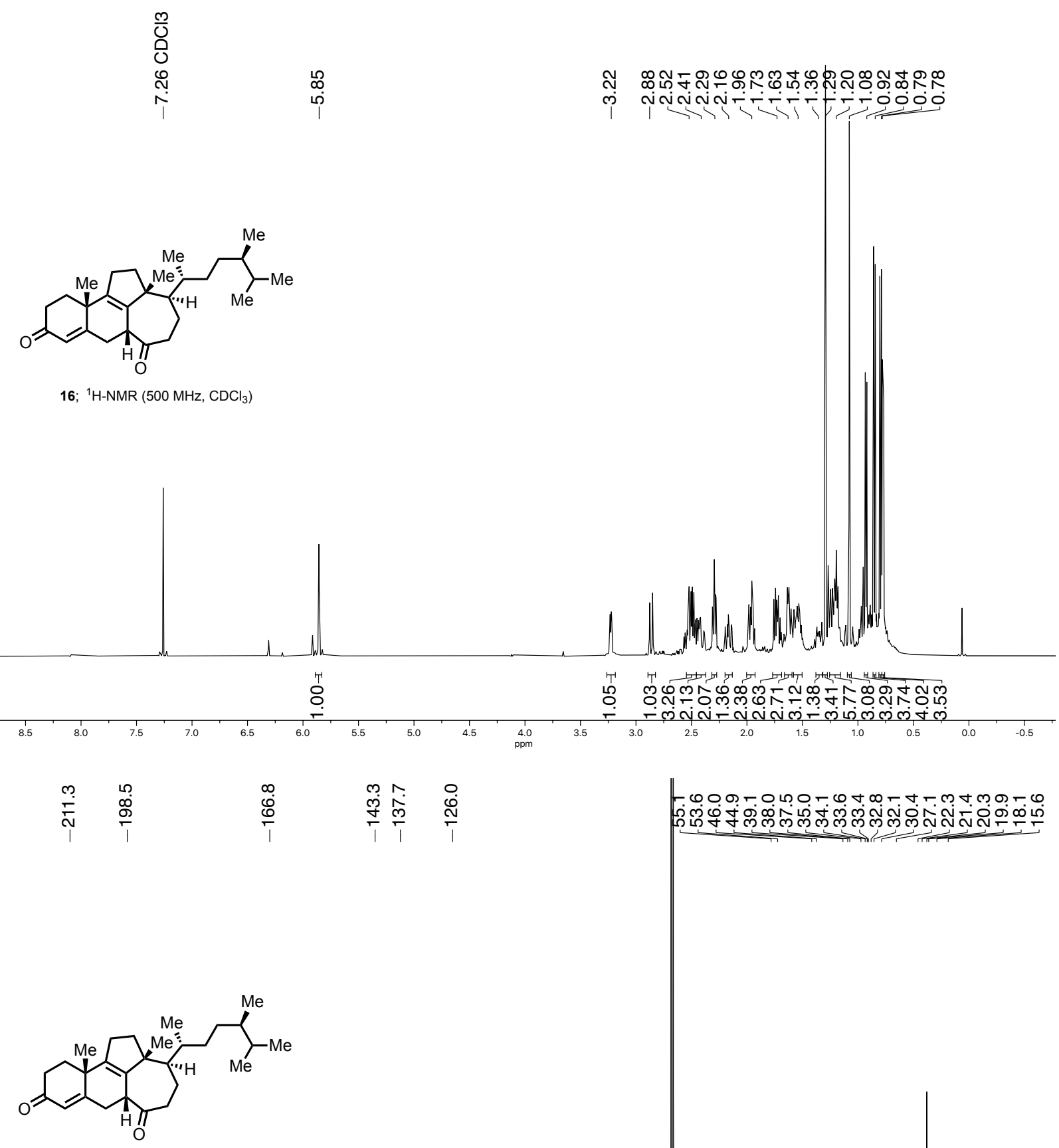

16; ${ }^{13} \mathrm{C}-\mathrm{NMR}\left(126 \mathrm{MHz}, \mathrm{CDCl}_{3}\right)$
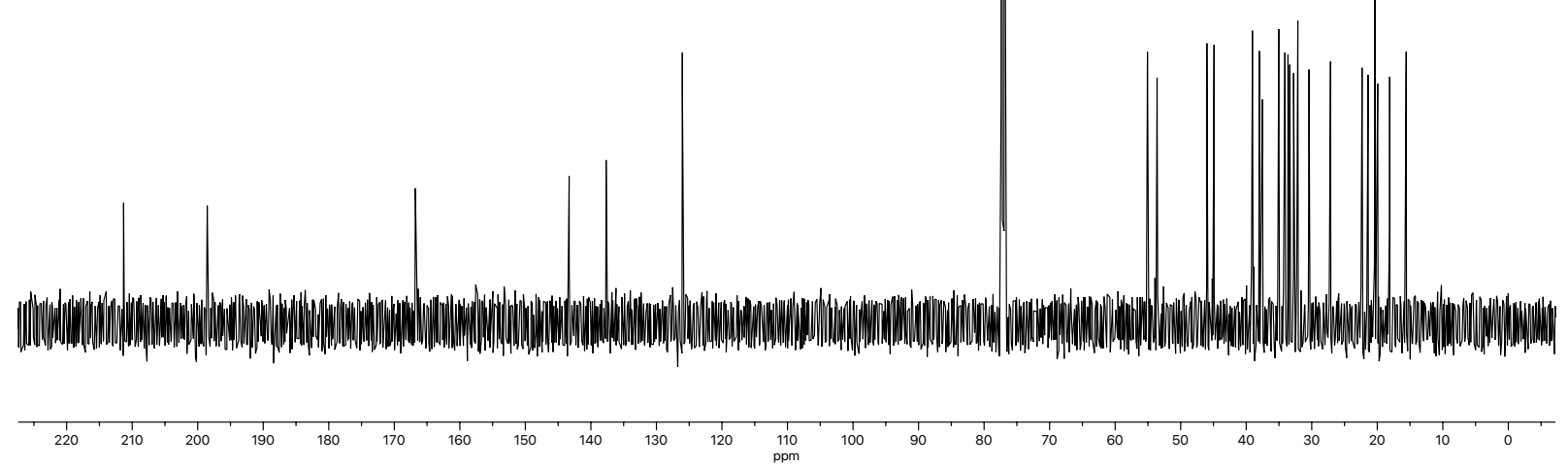

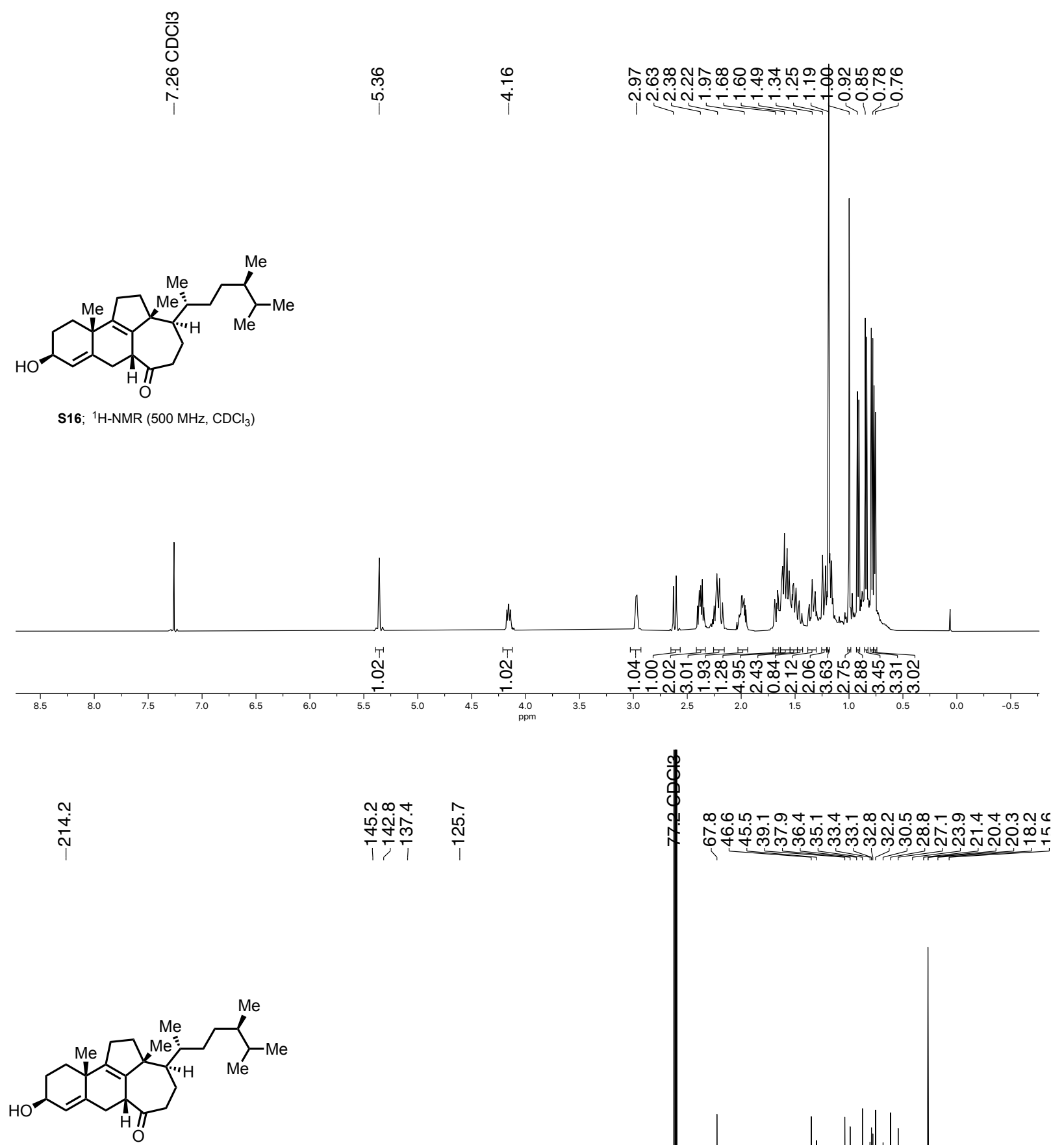

S16; ${ }^{13} \mathrm{C}-\mathrm{NMR}\left(126 \mathrm{MHz}, \mathrm{CDCl}_{3}\right)$

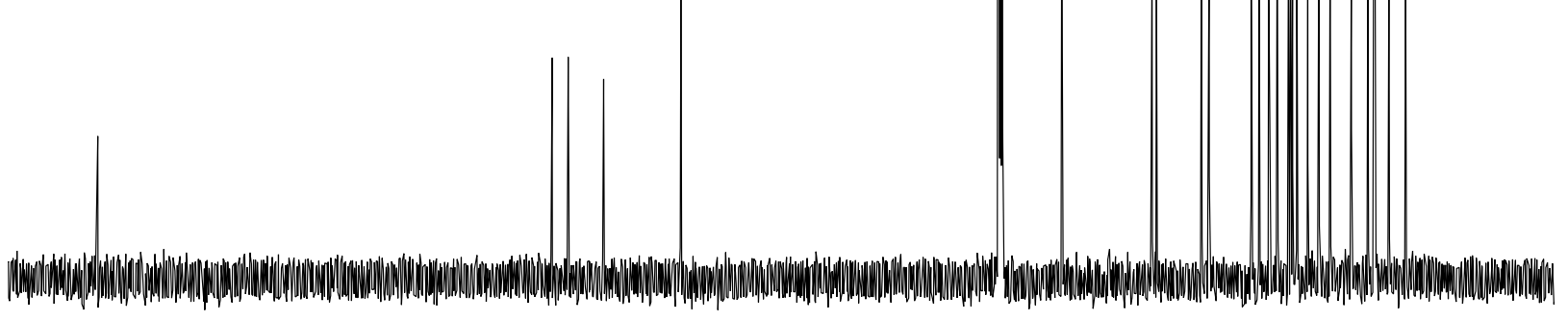

$\begin{array}{llllllllllll}220 & 210 & 200 & 190 & 180 & 170 & 160 & 150 & 140 & 130 & 120 & \begin{array}{r}110 \\ \text { ppm }\end{array}\end{array}$ 

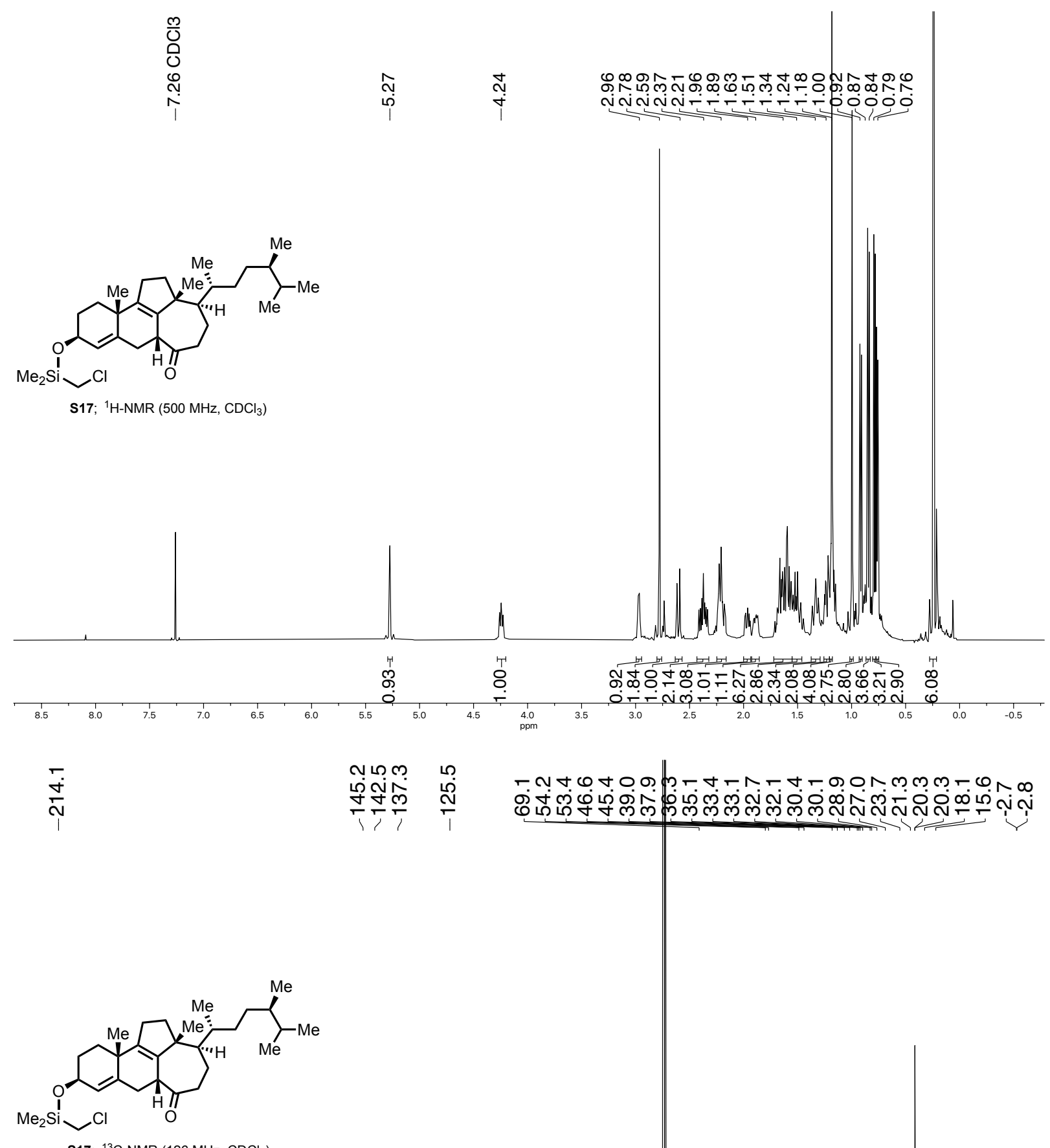

S17; ${ }^{13} \mathrm{C}-\mathrm{NMR}\left(126 \mathrm{MHz}, \mathrm{CDCl}_{3}\right)$

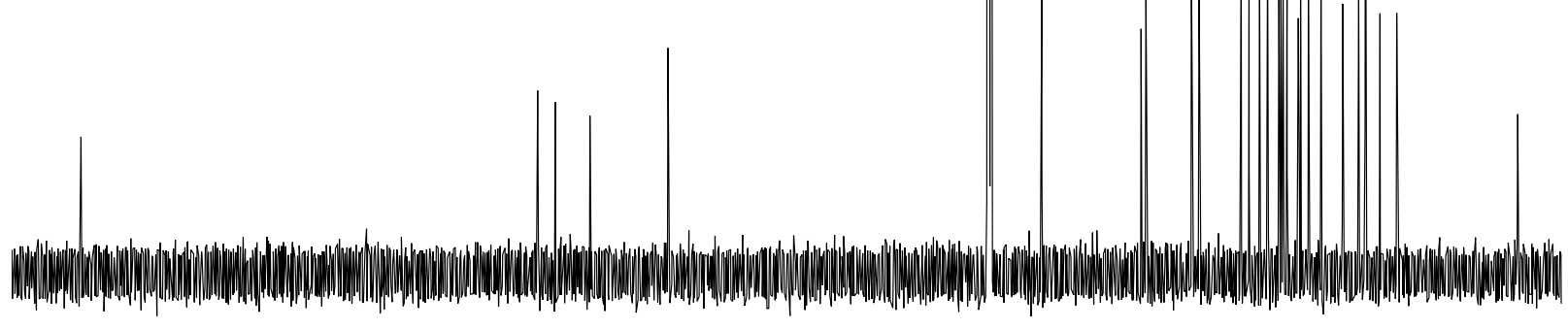

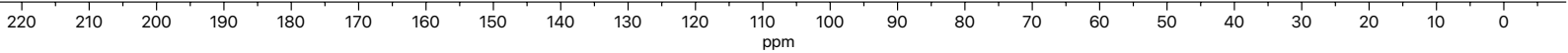




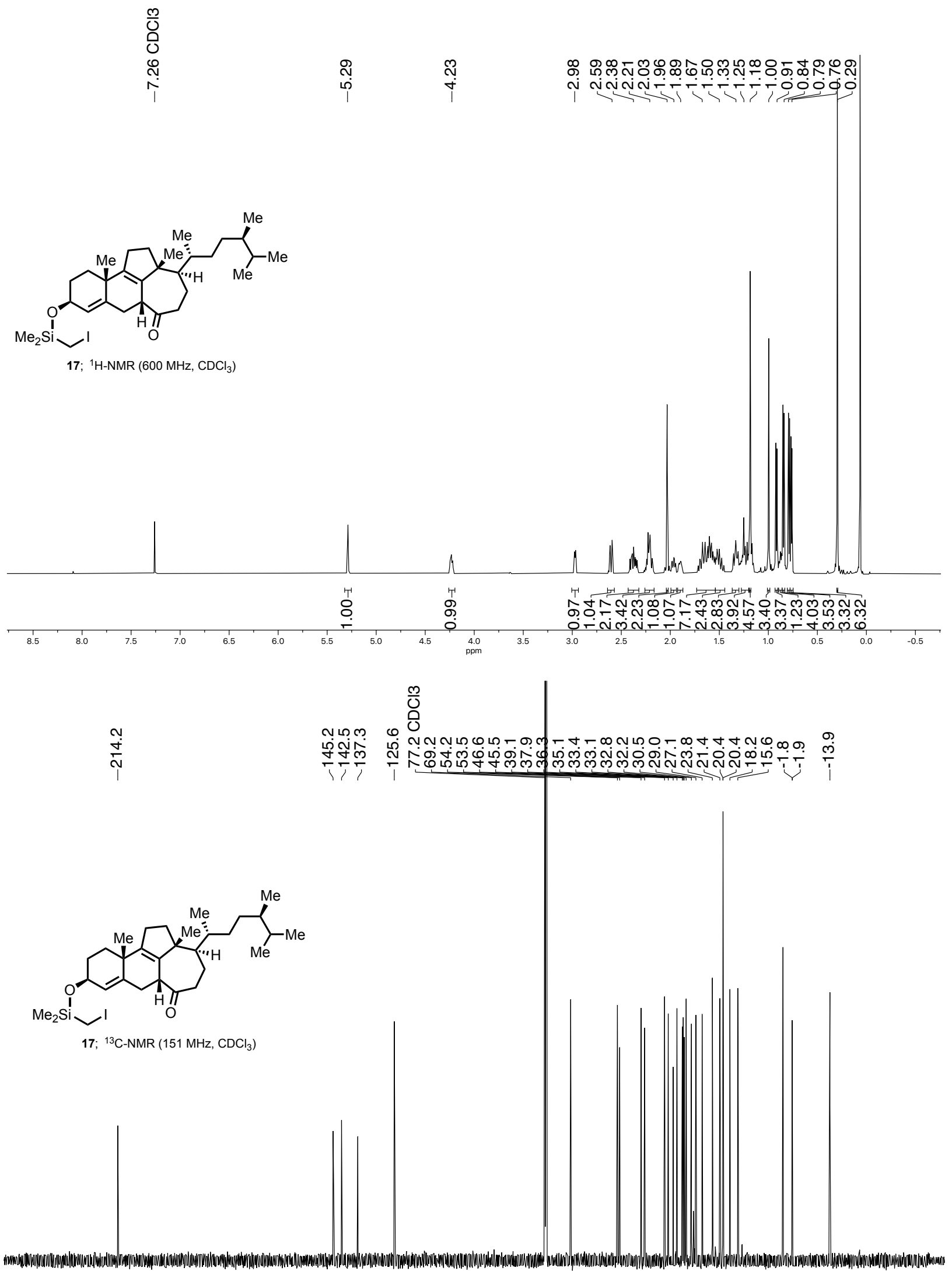

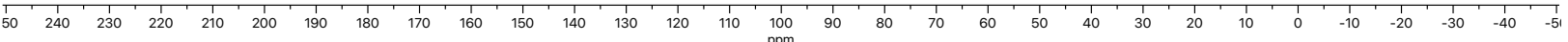




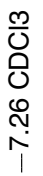

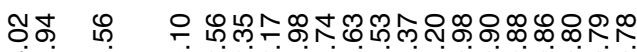

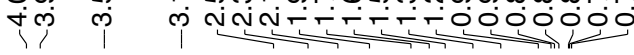

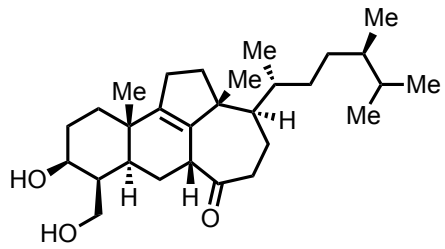

18; ${ }^{1} \mathrm{H}-\mathrm{NMR}\left(600 \mathrm{MHz}, \mathrm{CDCl}_{3}\right)$

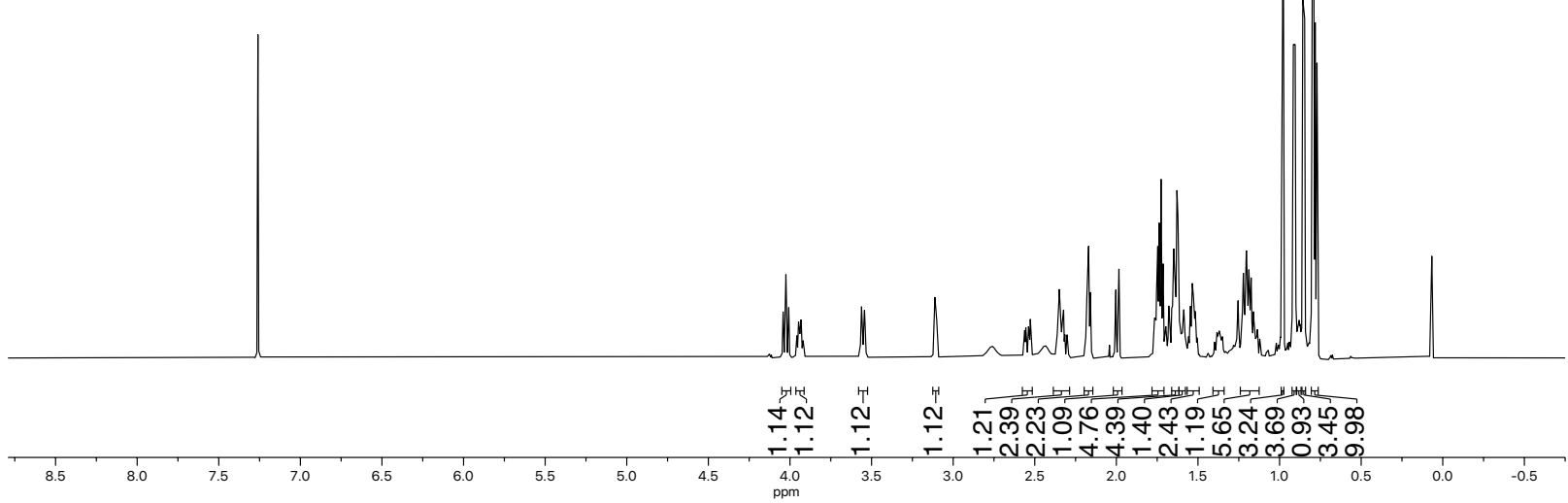

O

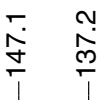

ง

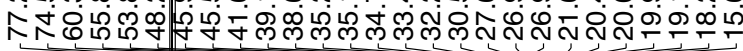

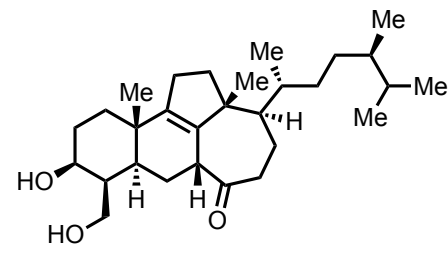

18; ${ }^{13} \mathrm{C}-\mathrm{NMR}\left(151 \mathrm{MHz}, \mathrm{CDCl}_{3}\right)$
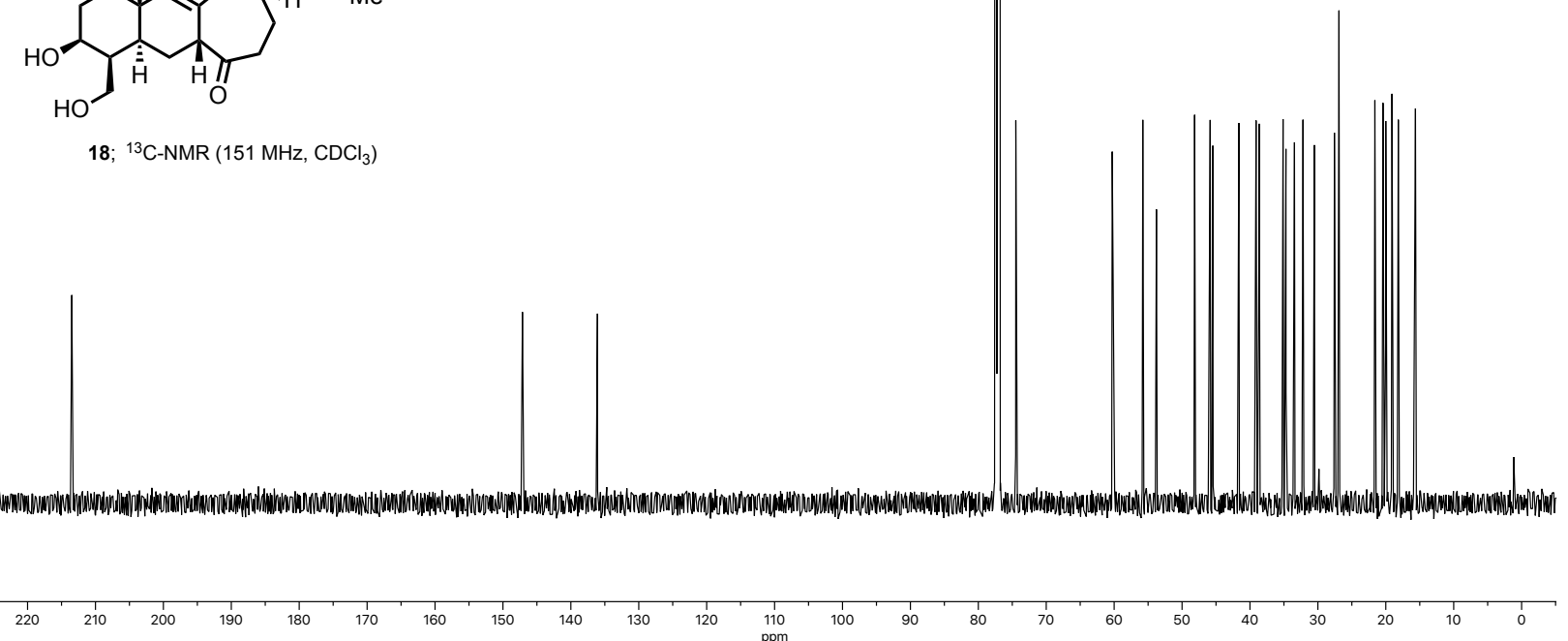

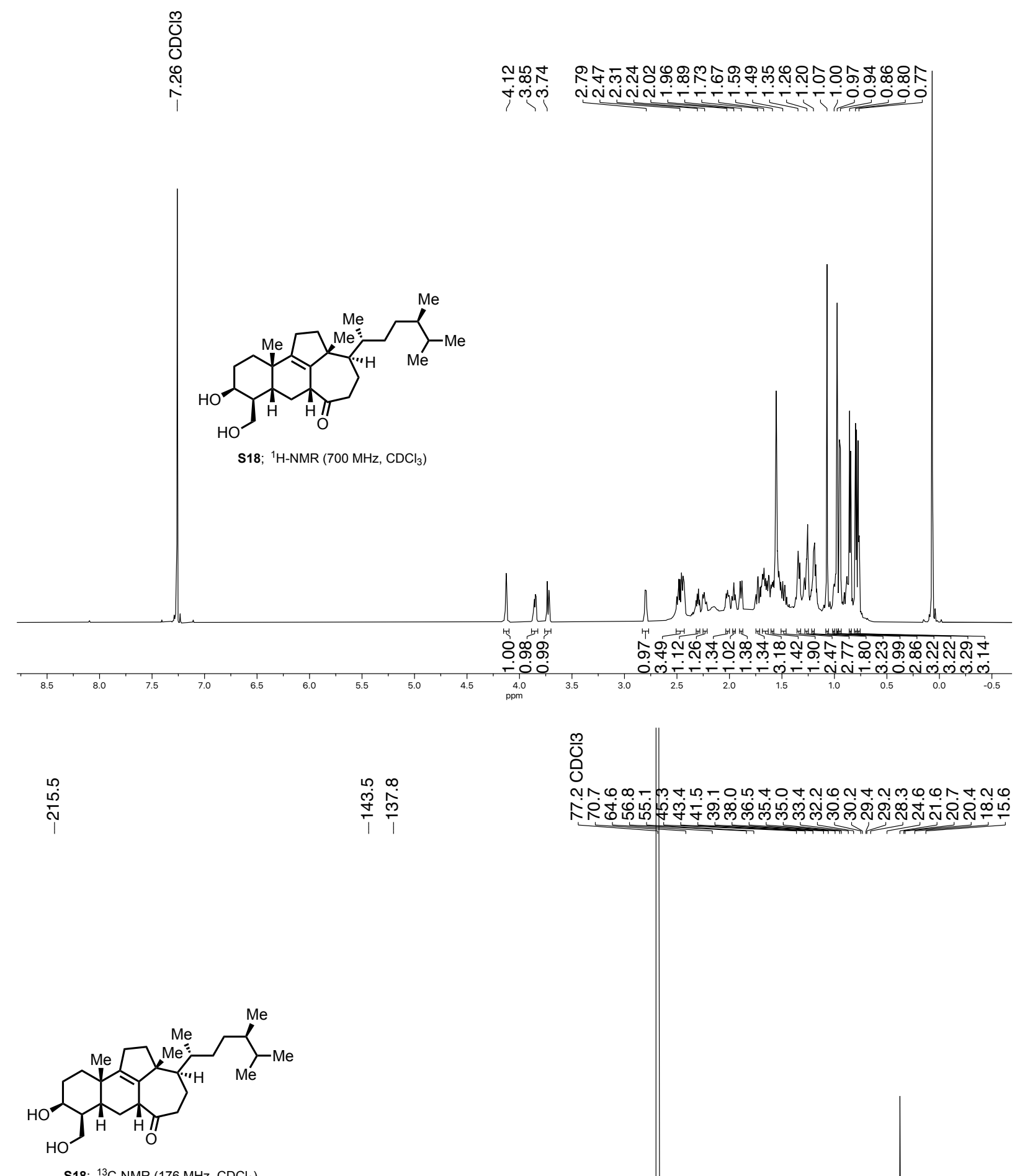

S18; ${ }^{13} \mathrm{C}-\mathrm{NMR}\left(176 \mathrm{MHz}, \mathrm{CDCl}_{3}\right)$
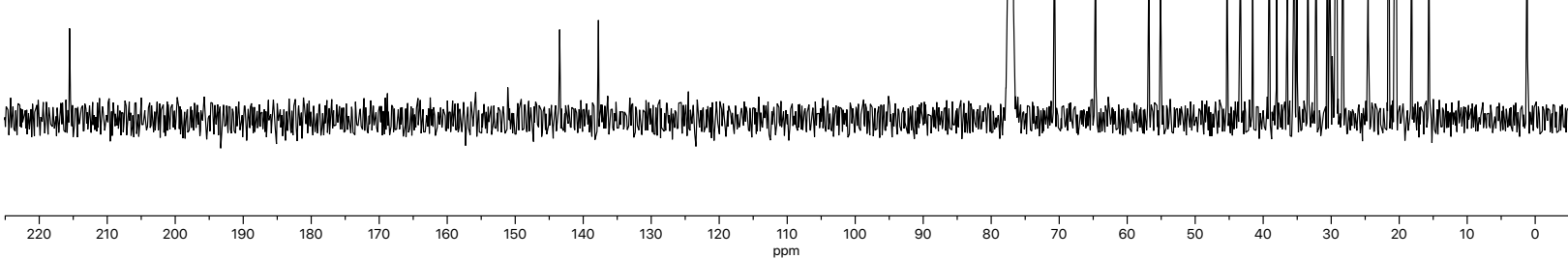

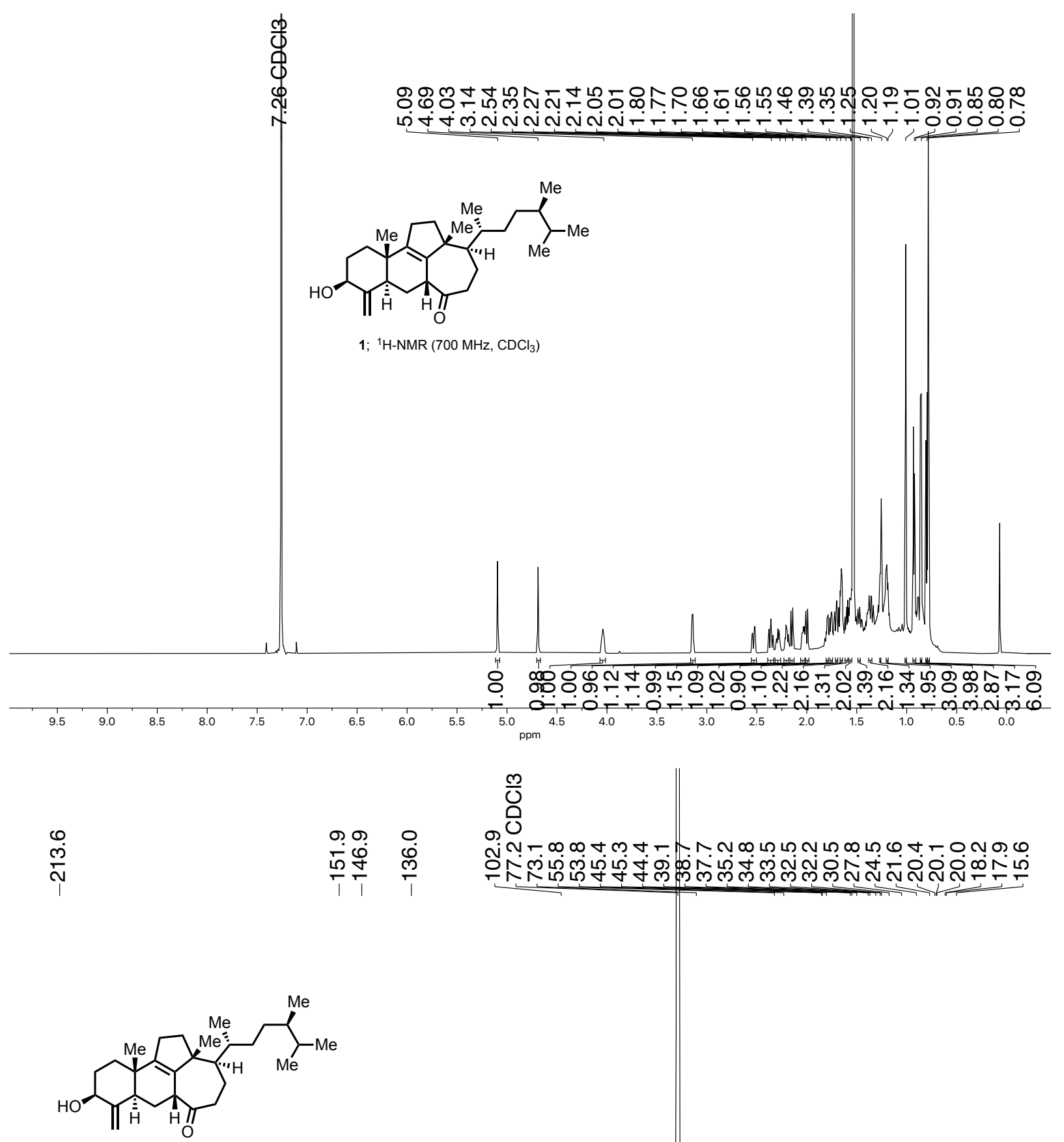

1; ${ }^{13} \mathrm{C}-\mathrm{NMR}\left(176 \mathrm{MHz}, \mathrm{CDCl}_{3}\right.$ )
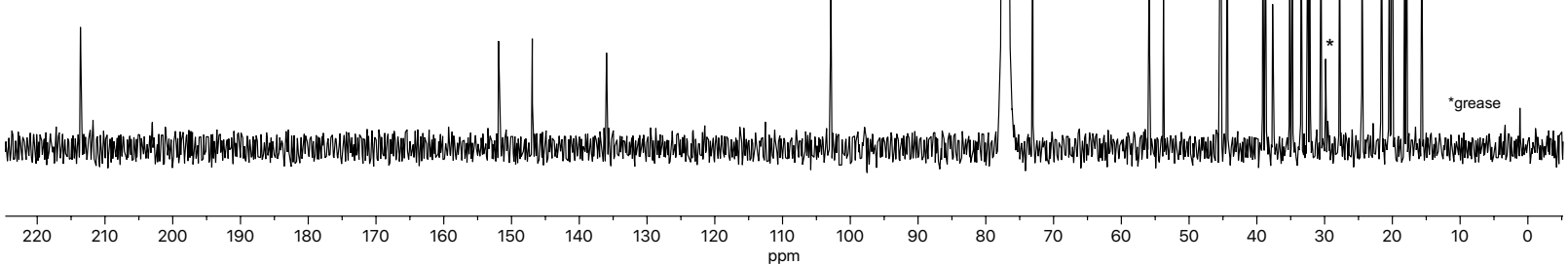
$\frac{m}{0}$
0
0
$\stackrel{0}{i}$
$i$
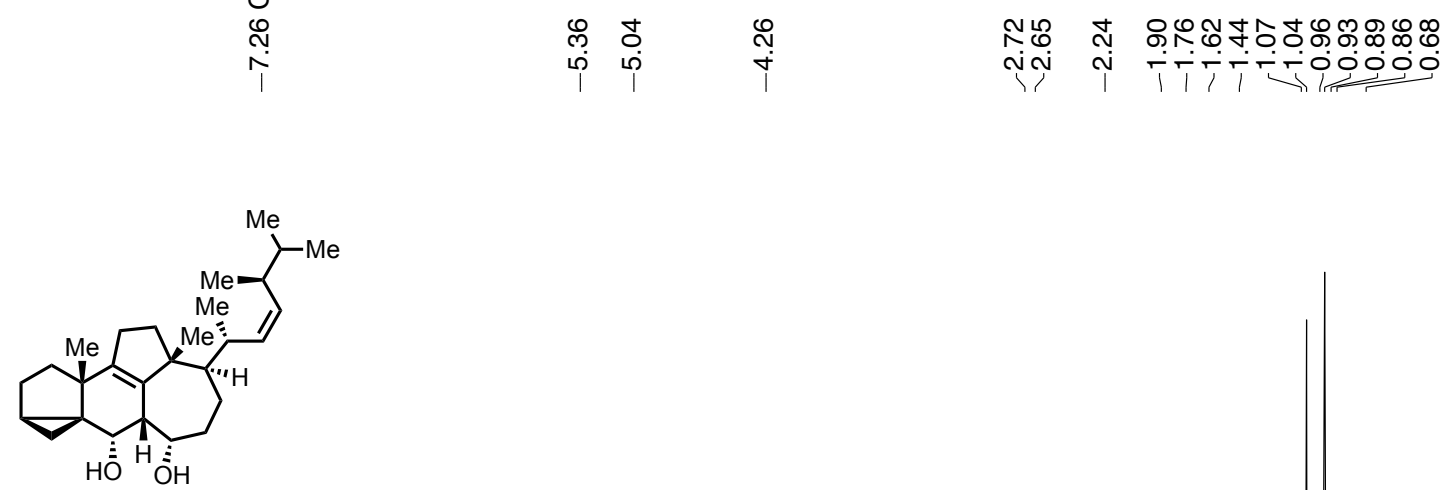

S19; ${ }^{1} \mathrm{H}-\mathrm{NMR}\left(400 \mathrm{MHz}, \mathrm{CDCl}_{3}\right)$

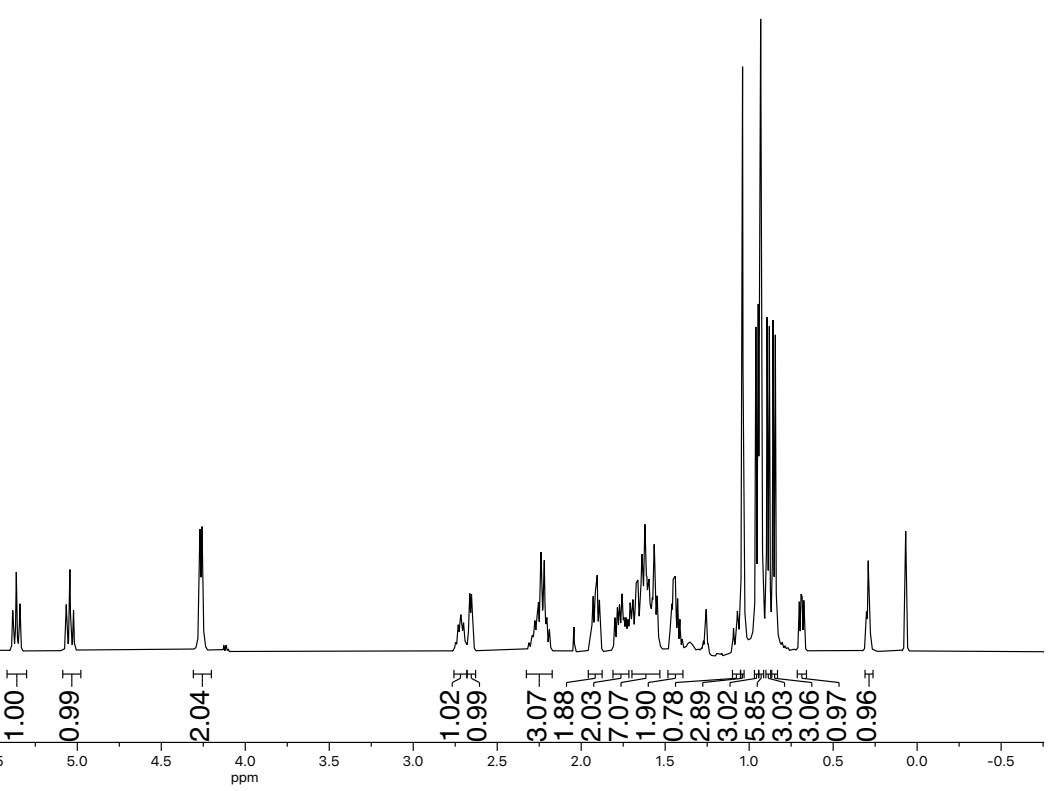

우음

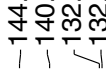
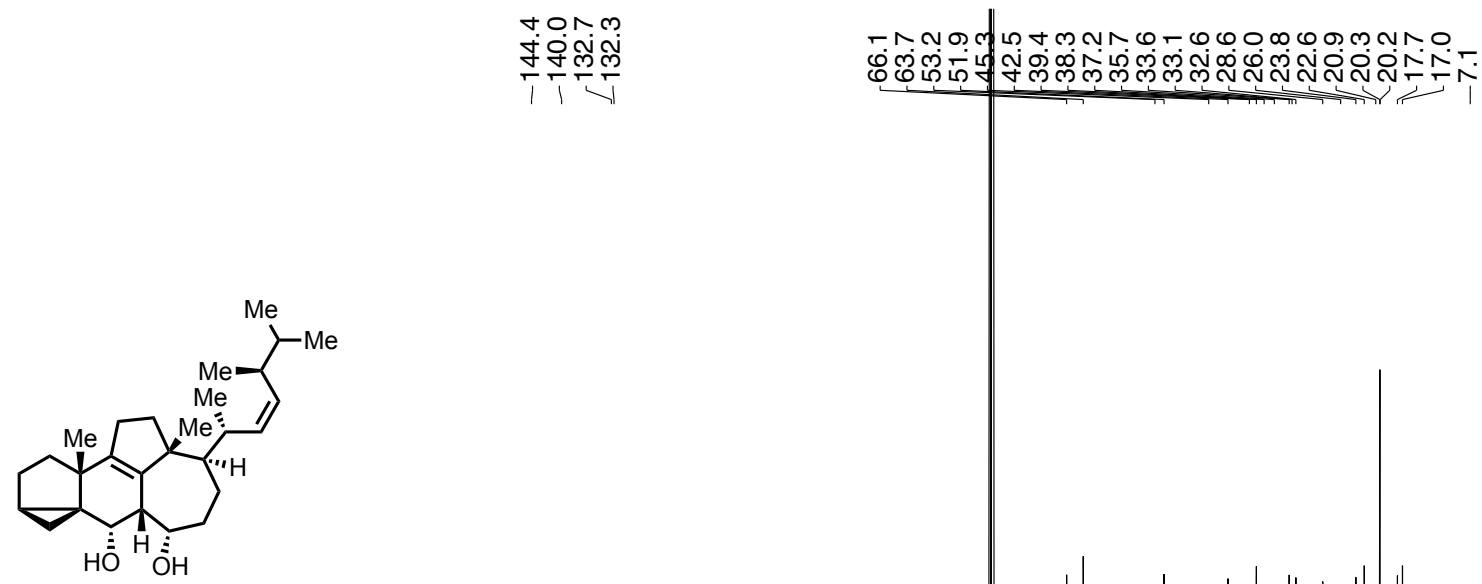

S19; ${ }^{13} \mathrm{C}-\mathrm{NMR}\left(126 \mathrm{MHz}, \mathrm{CDCl}_{3}\right)$
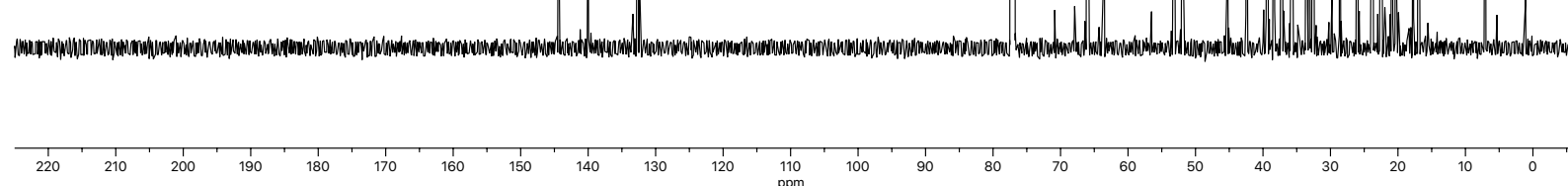


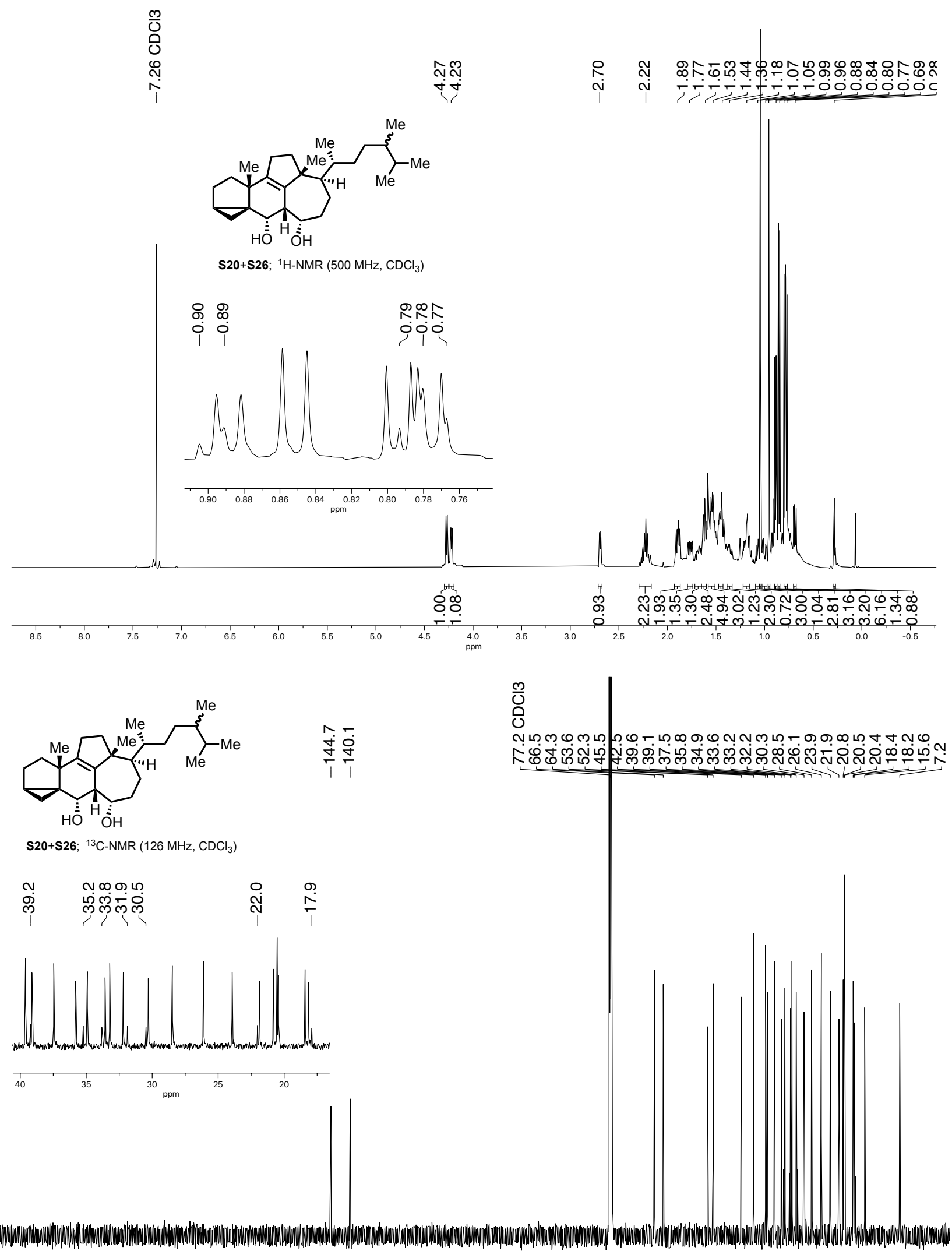

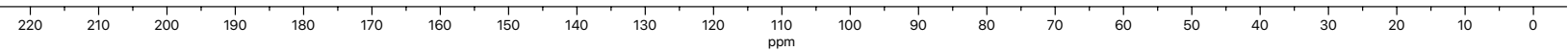




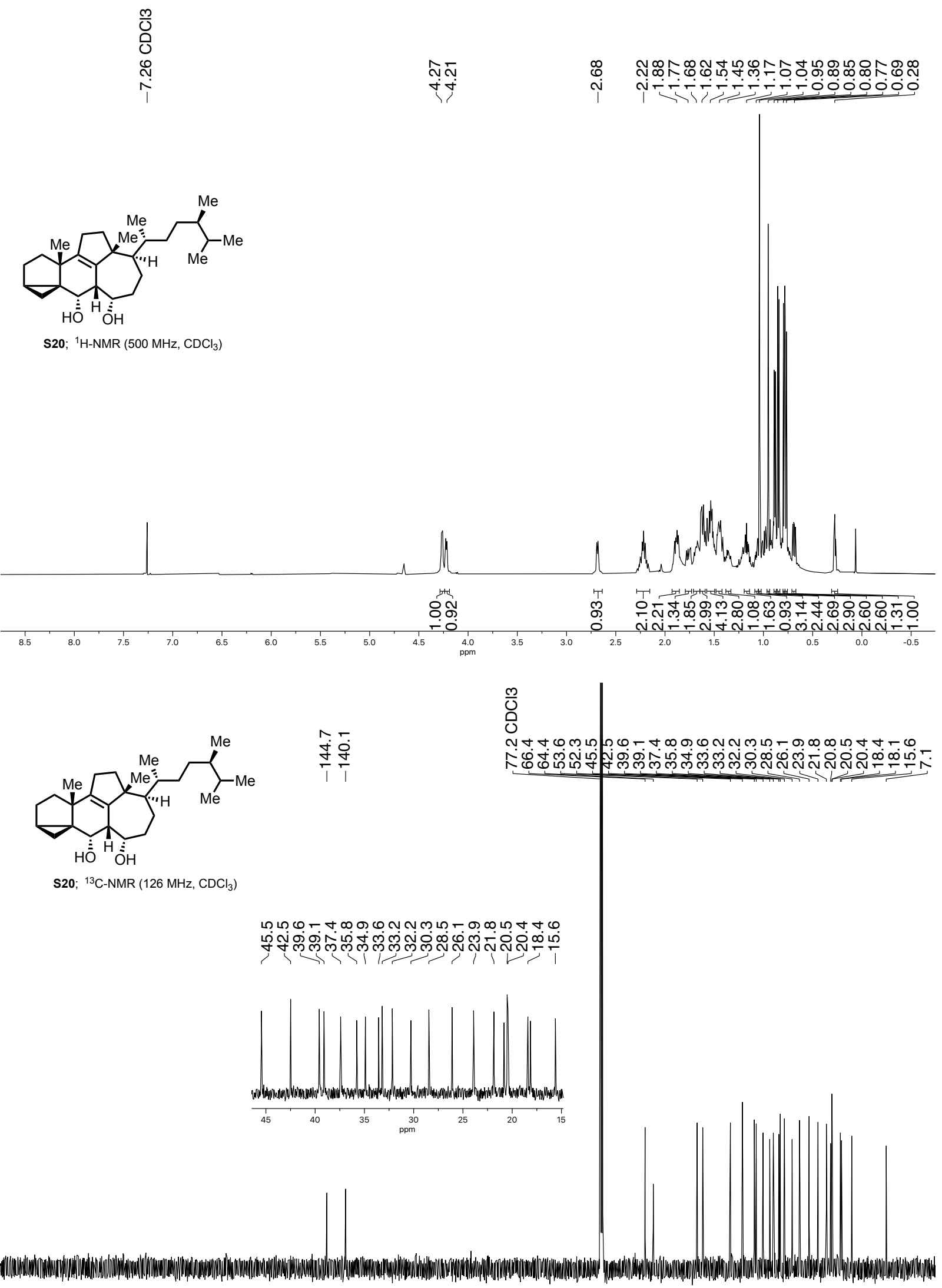

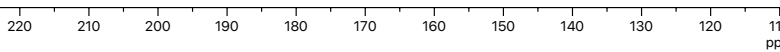



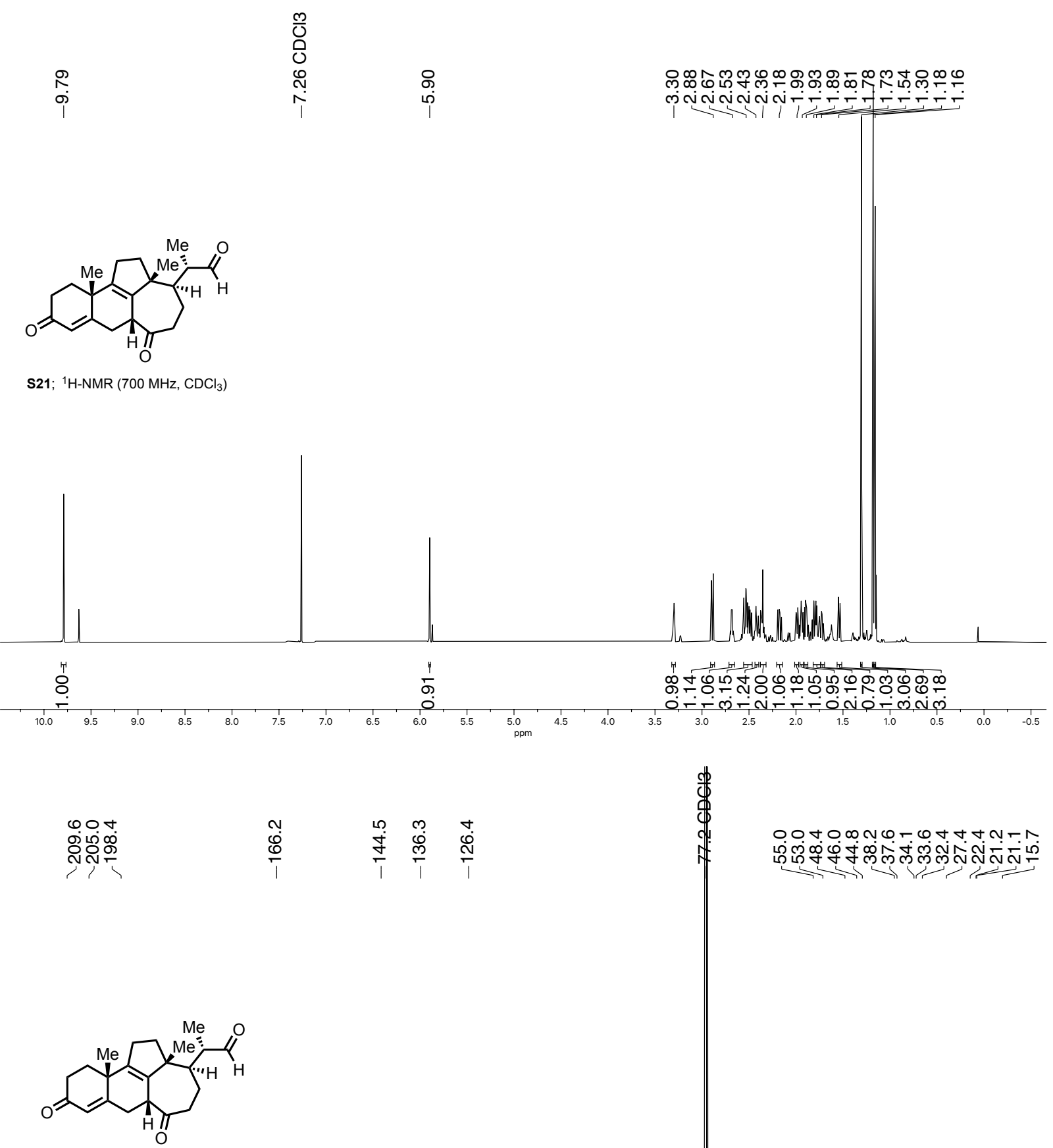

S21; ${ }^{13} \mathrm{C}-\mathrm{NMR}\left(176 \mathrm{MHz}, \mathrm{CDCl}_{3}\right)$
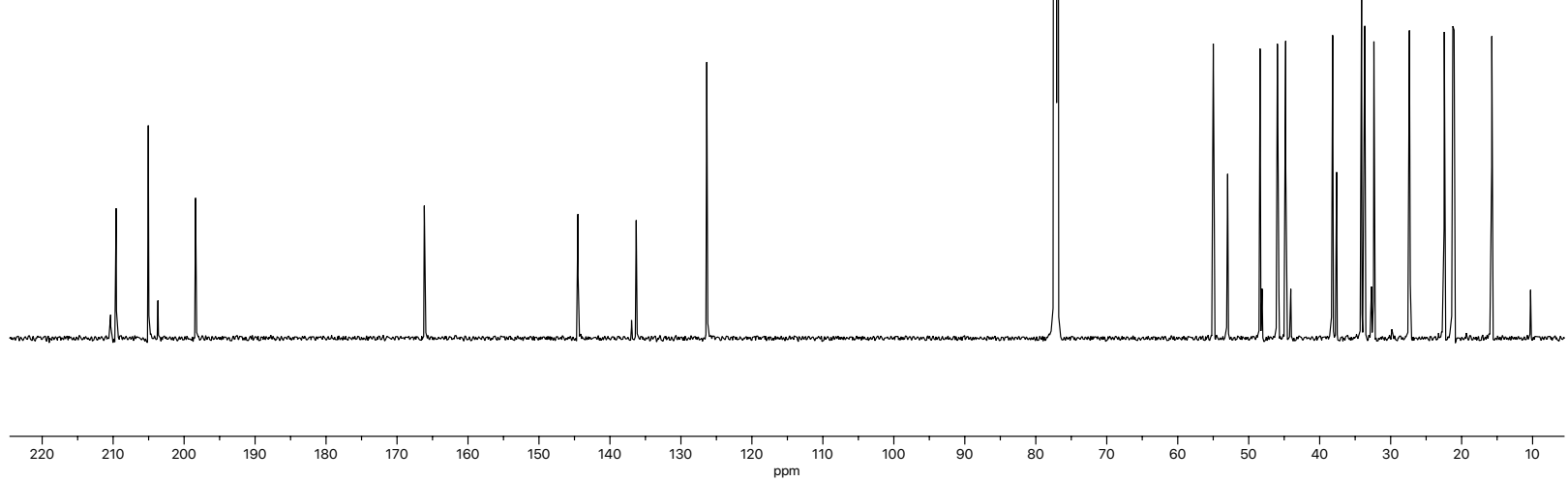

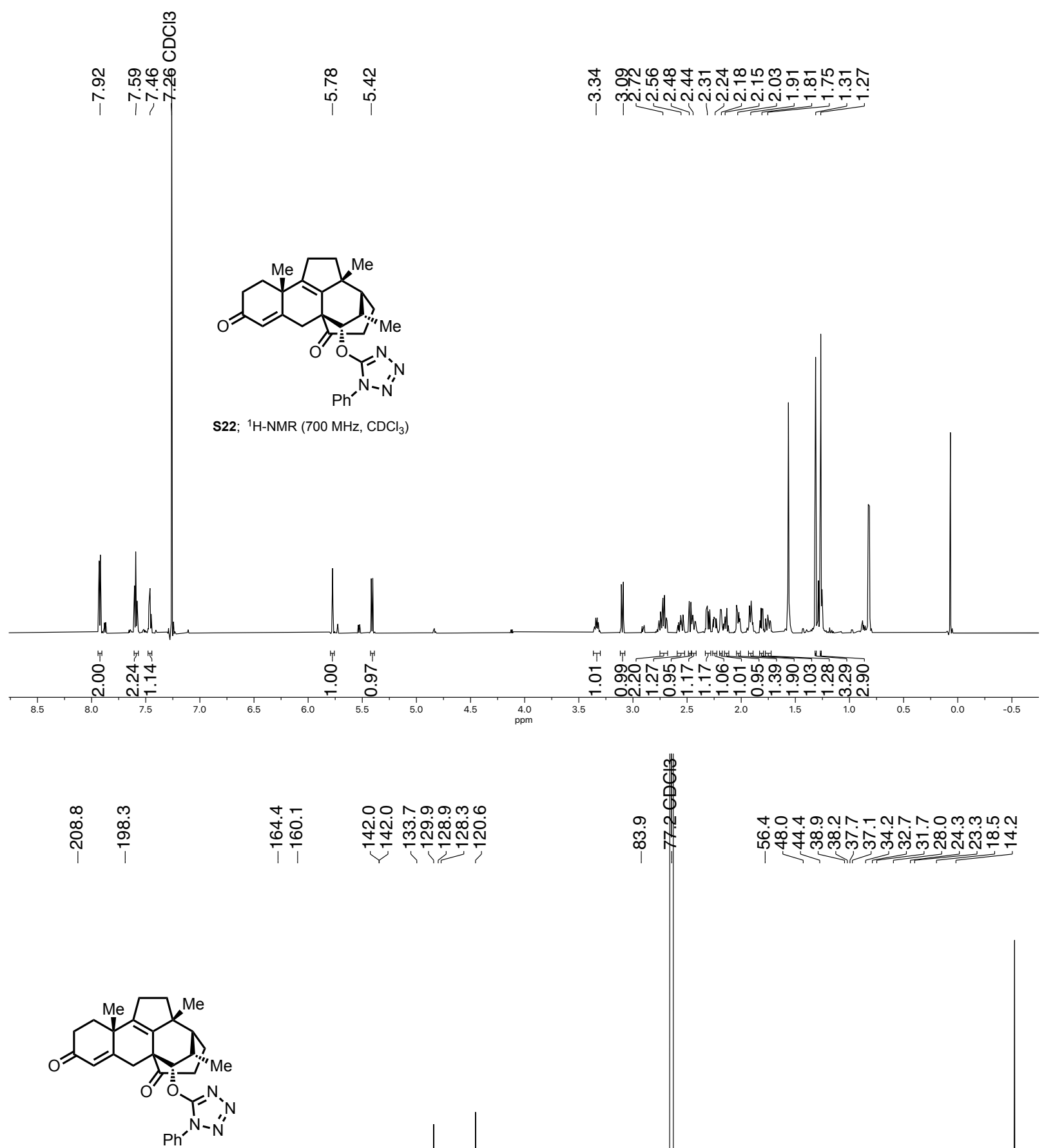

S22; ${ }^{13} \mathrm{C}-\mathrm{NMR}\left(176 \mathrm{MHz}, \mathrm{CDCl}_{3}\right)$ 


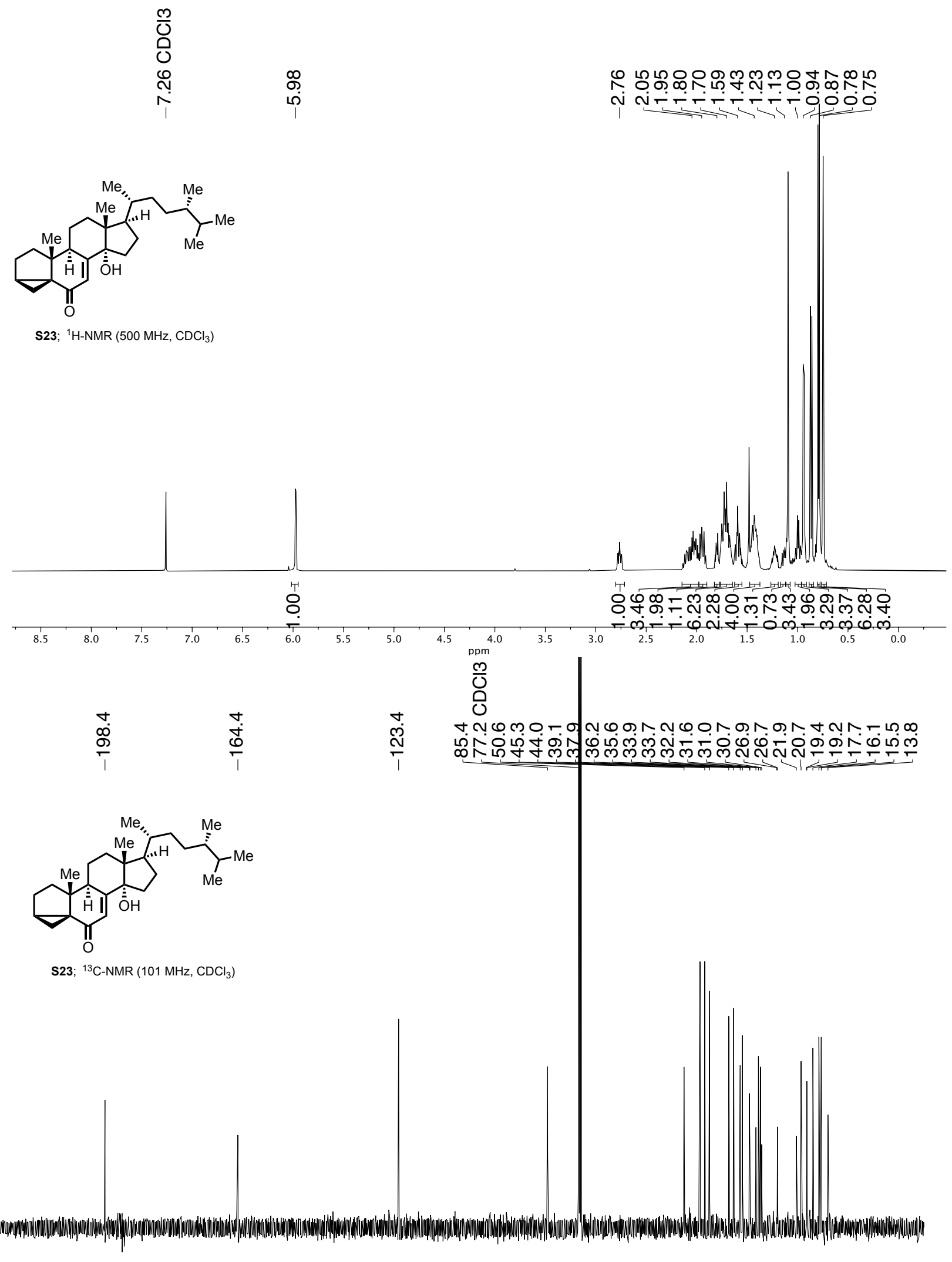

$\begin{array}{lllllllllllllllllllllllll}|1| & 1 \\ 220 & 210 & 200 & 190 & 180 & 170 & 160 & 150 & 140 & 130 & 120 & 110 & 100 & 90 & 80 & 70 & 60 & 50 & 40 & 30 & 20 & 10 & 0 & -11\end{array}$ 


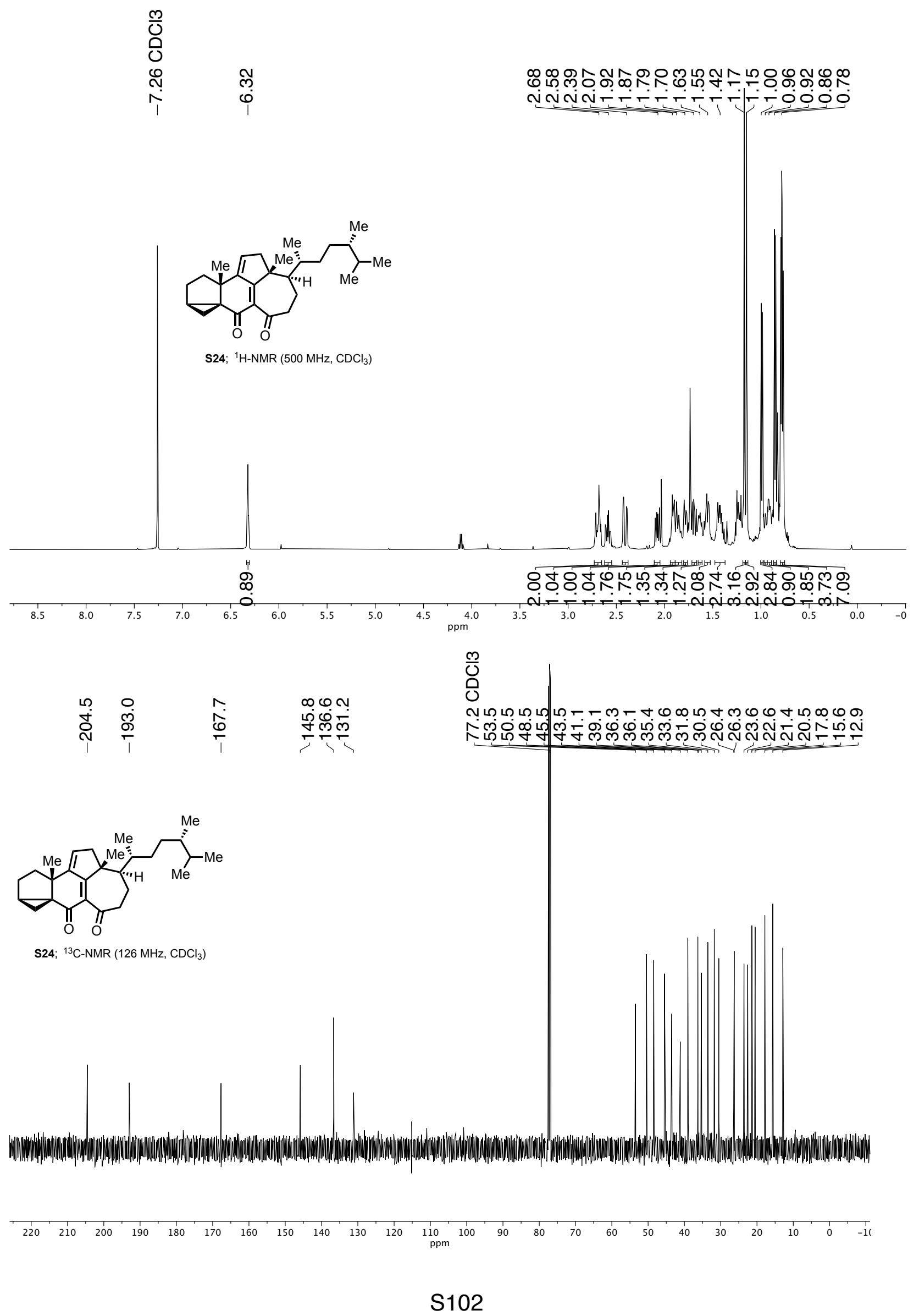



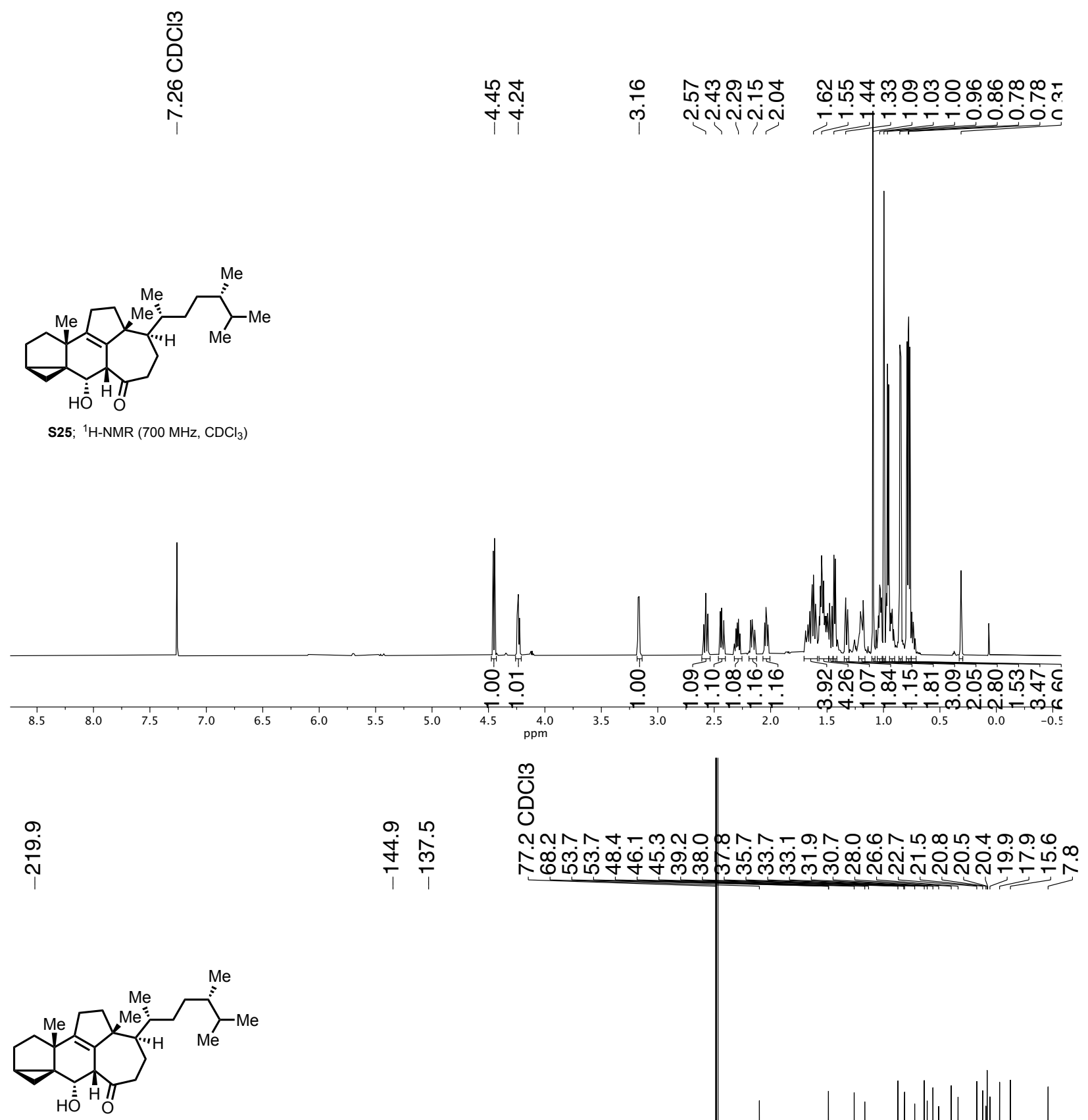

S25; ${ }^{13} \mathrm{C}-\mathrm{NMR}\left(176 \mathrm{MHz}, \mathrm{CDCl}_{3}\right)$
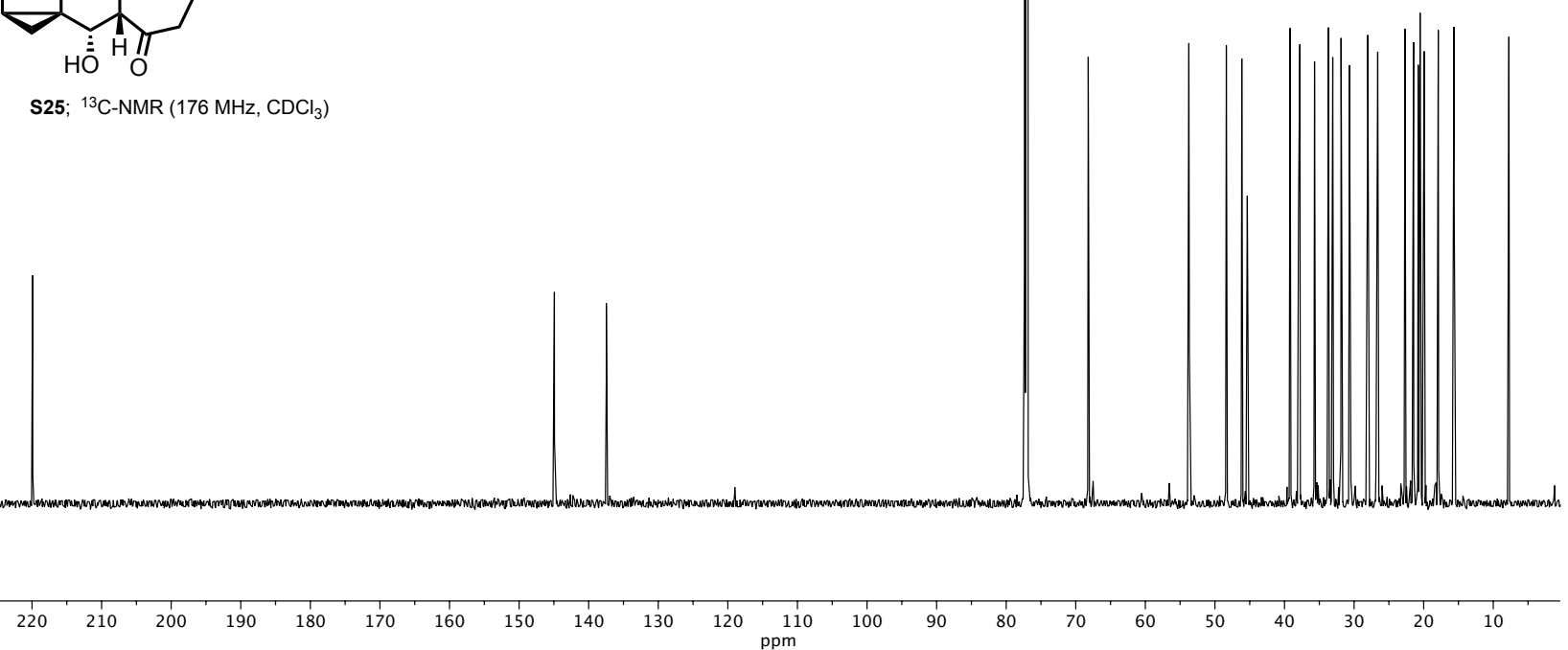

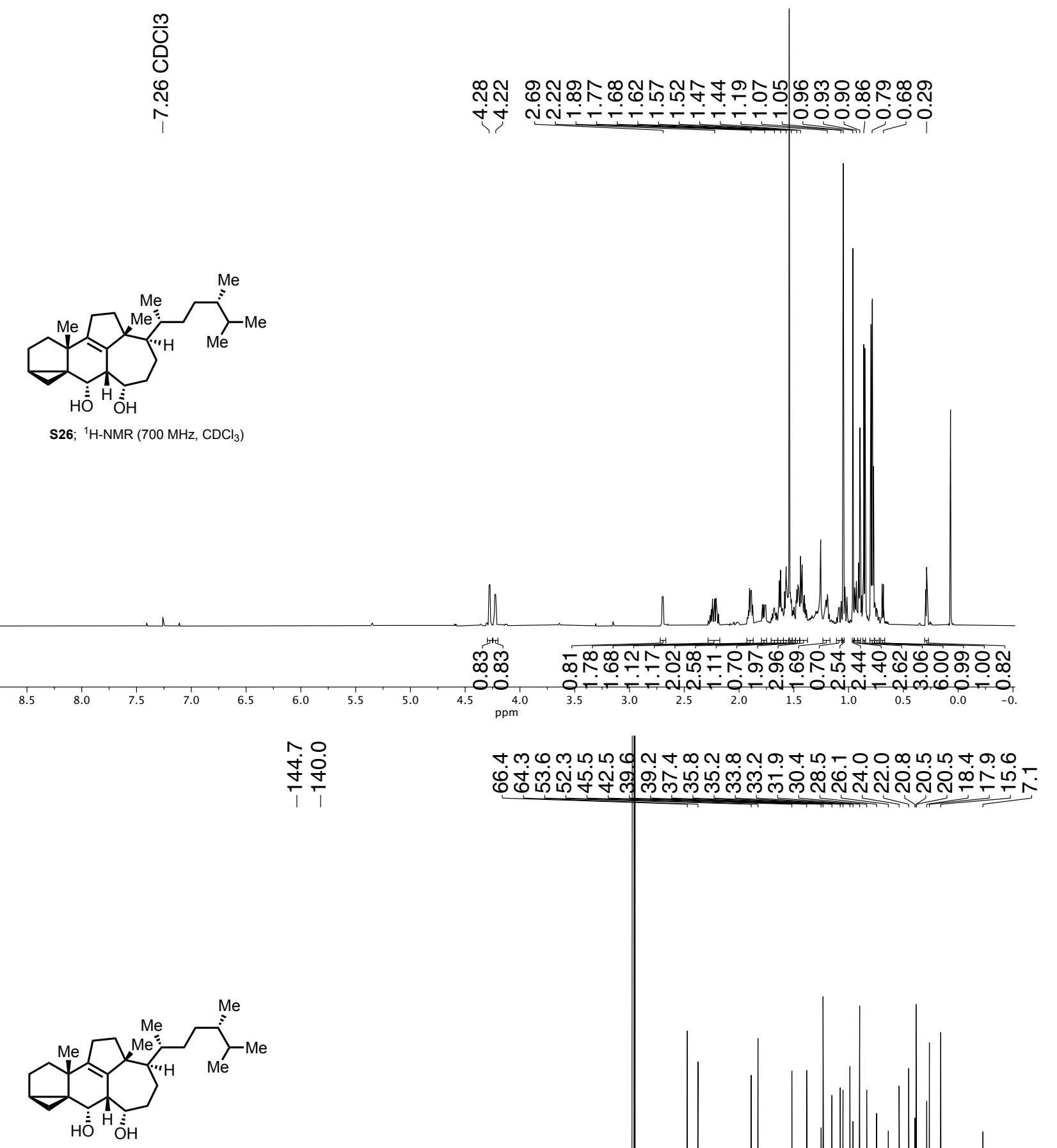

S26; ${ }^{13} \mathrm{C}-\mathrm{NMR}\left(151 \mathrm{MHz}, \mathrm{CDCl}_{3}\right)$

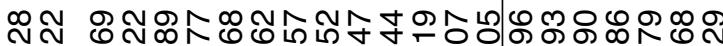

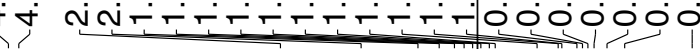

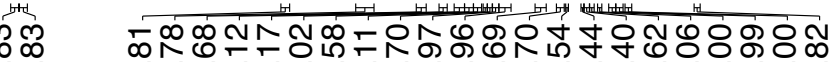

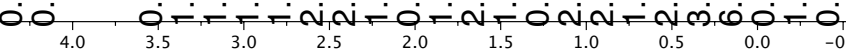

$\forall$ แ

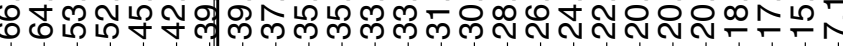

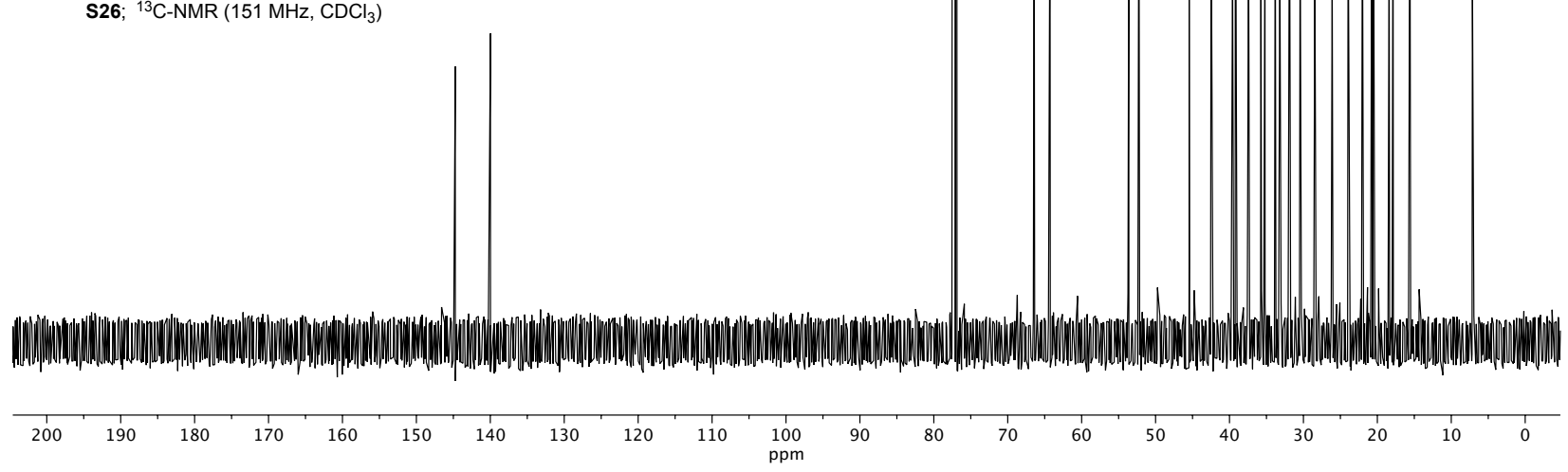



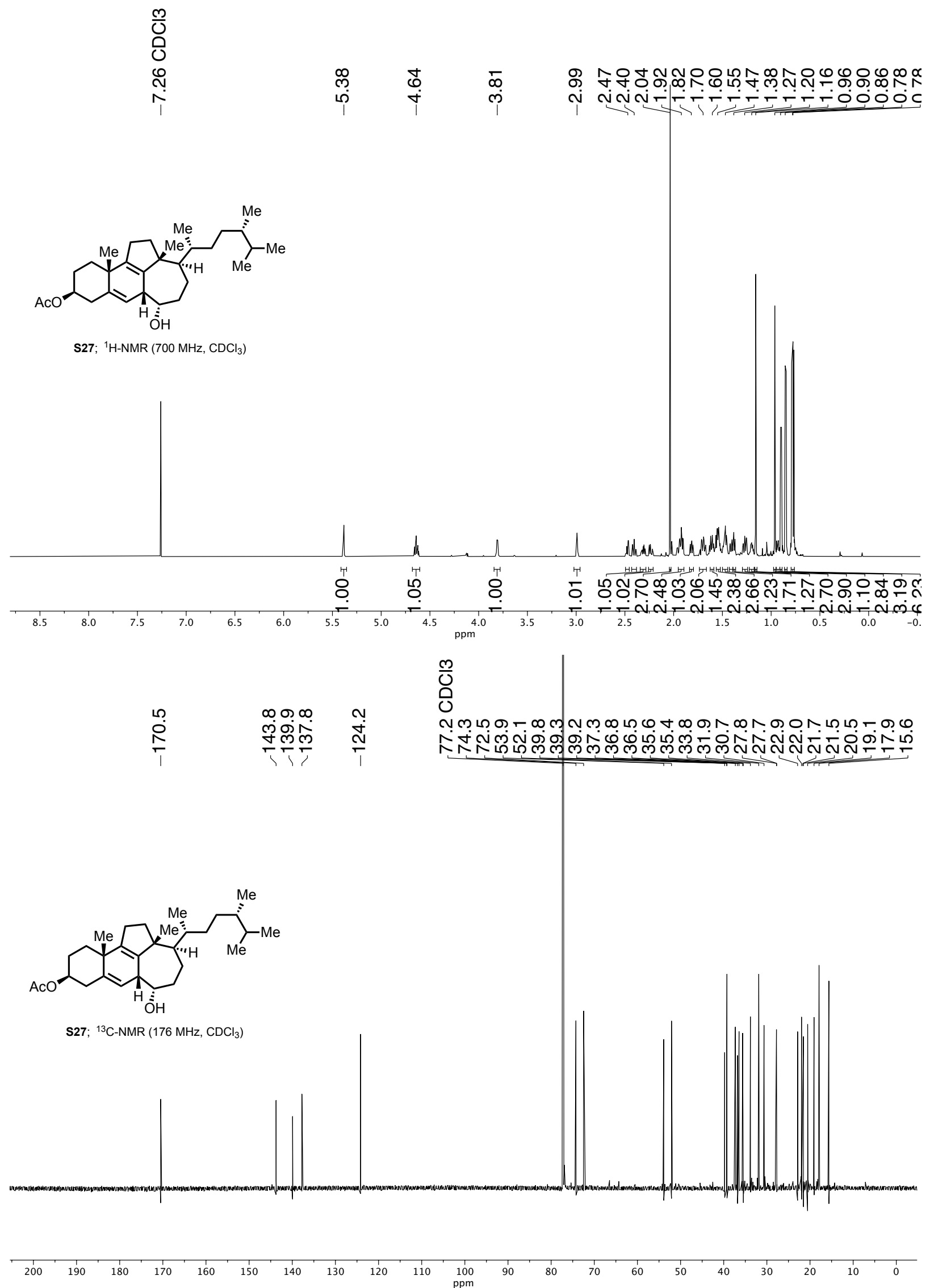

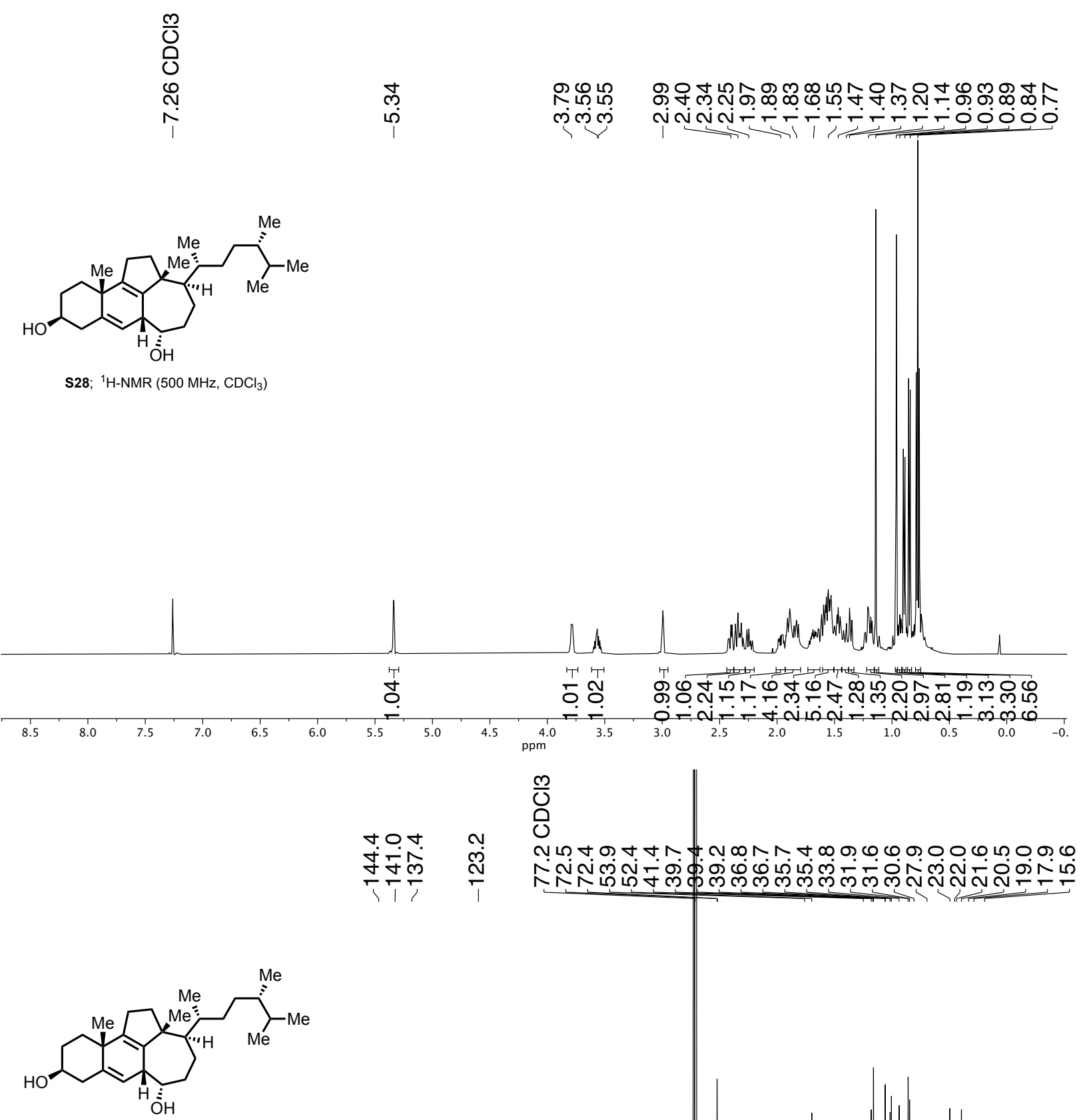

S28; ${ }^{13} \mathrm{C}-\mathrm{NMR}\left(126 \mathrm{MHz}, \mathrm{CDCl}_{3}\right)$

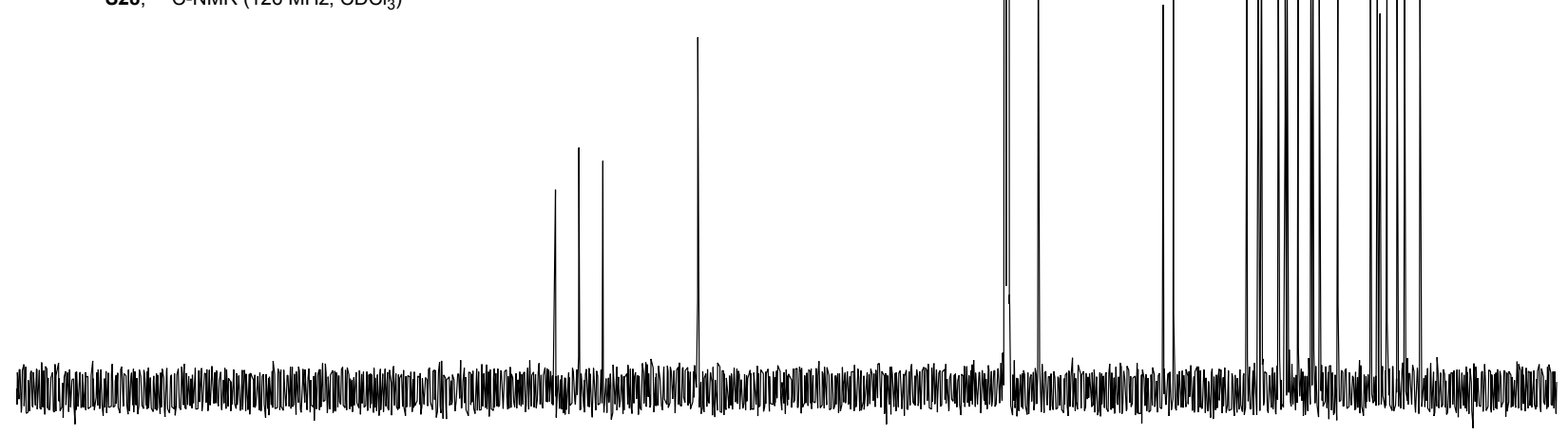

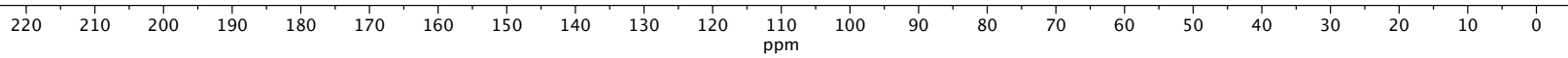




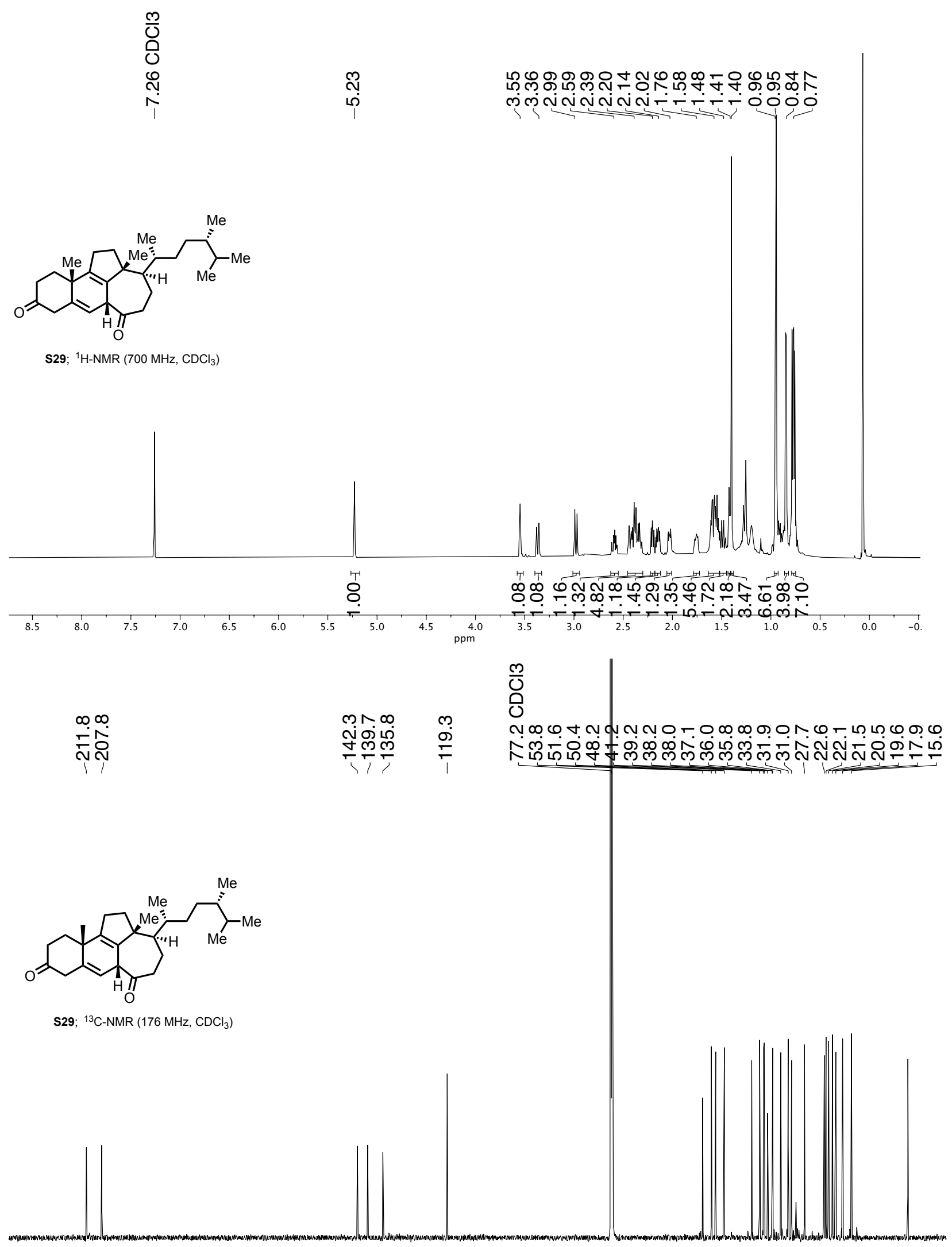

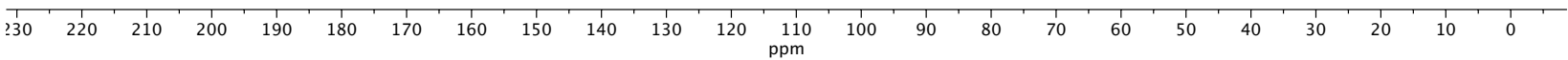


$\frac{m}{0}$
0
0
$\stackrel{0}{N}$
।

$\infty$
$\infty$
10

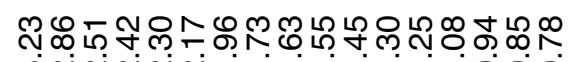

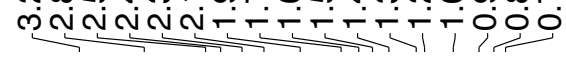

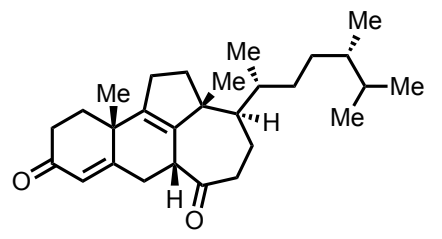

s30; ${ }^{1} \mathrm{H}-\mathrm{NMR}\left(700 \mathrm{MHz}, \mathrm{CDCl}_{3}\right)$

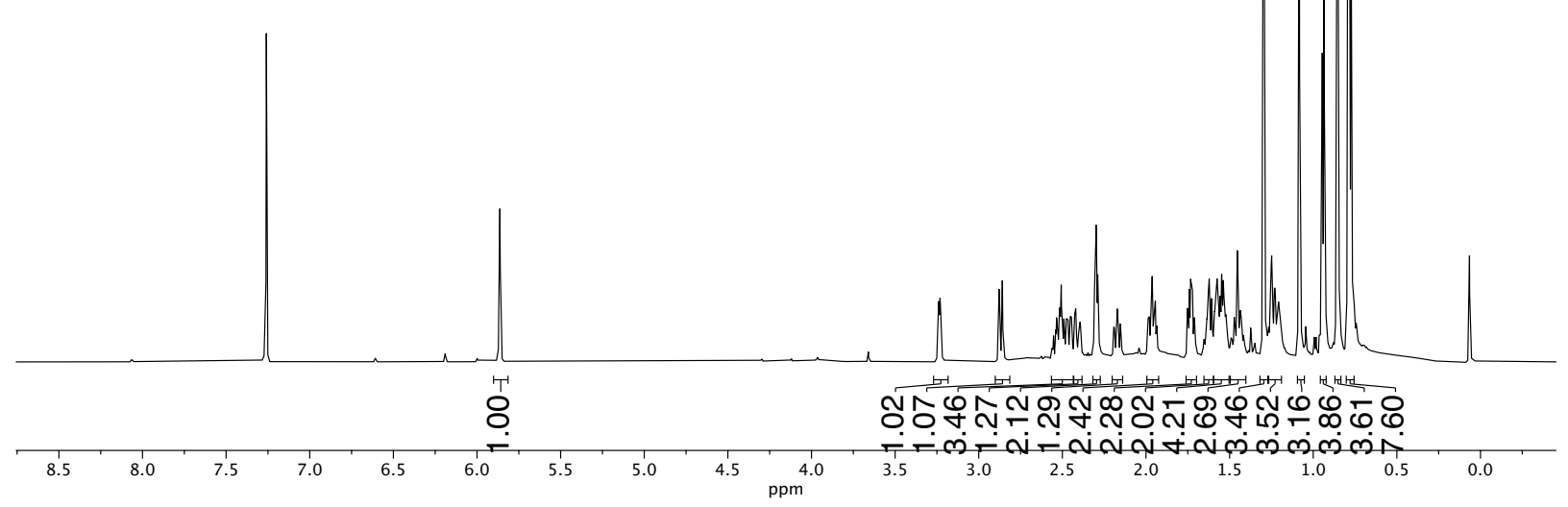

$\frac{m}{0}$

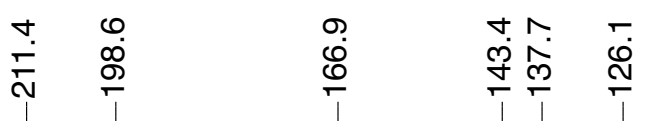

NT-

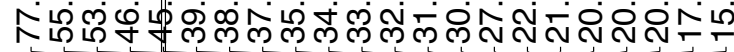

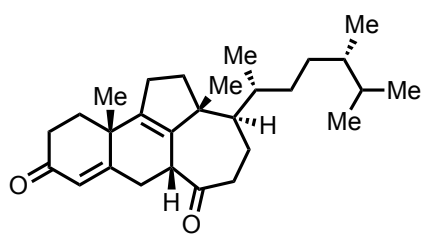

S30; ${ }^{13} \mathrm{C}-\mathrm{NMR}\left(176 \mathrm{MHz}, \mathrm{CDCl}_{3}\right)$

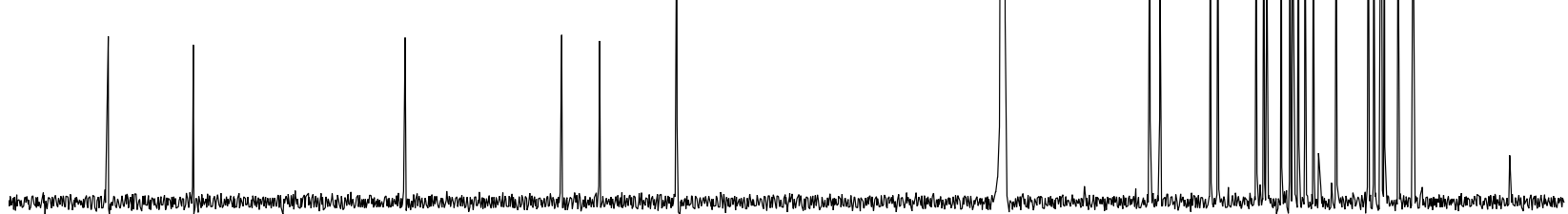

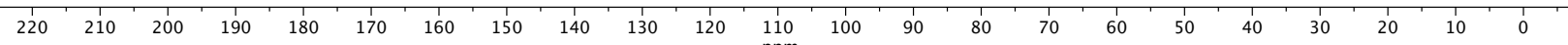




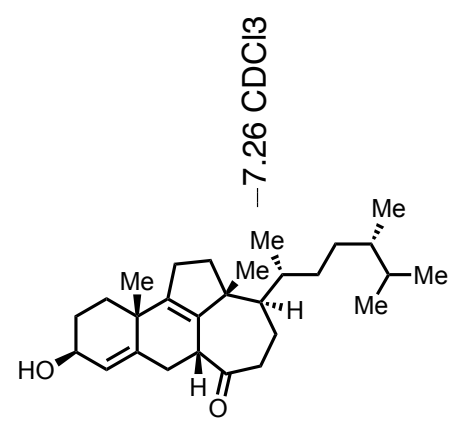

S31; ${ }^{1} \mathrm{H}-\mathrm{NMR}\left(700 \mathrm{MHz}, \mathrm{CDCl}_{3}\right)$
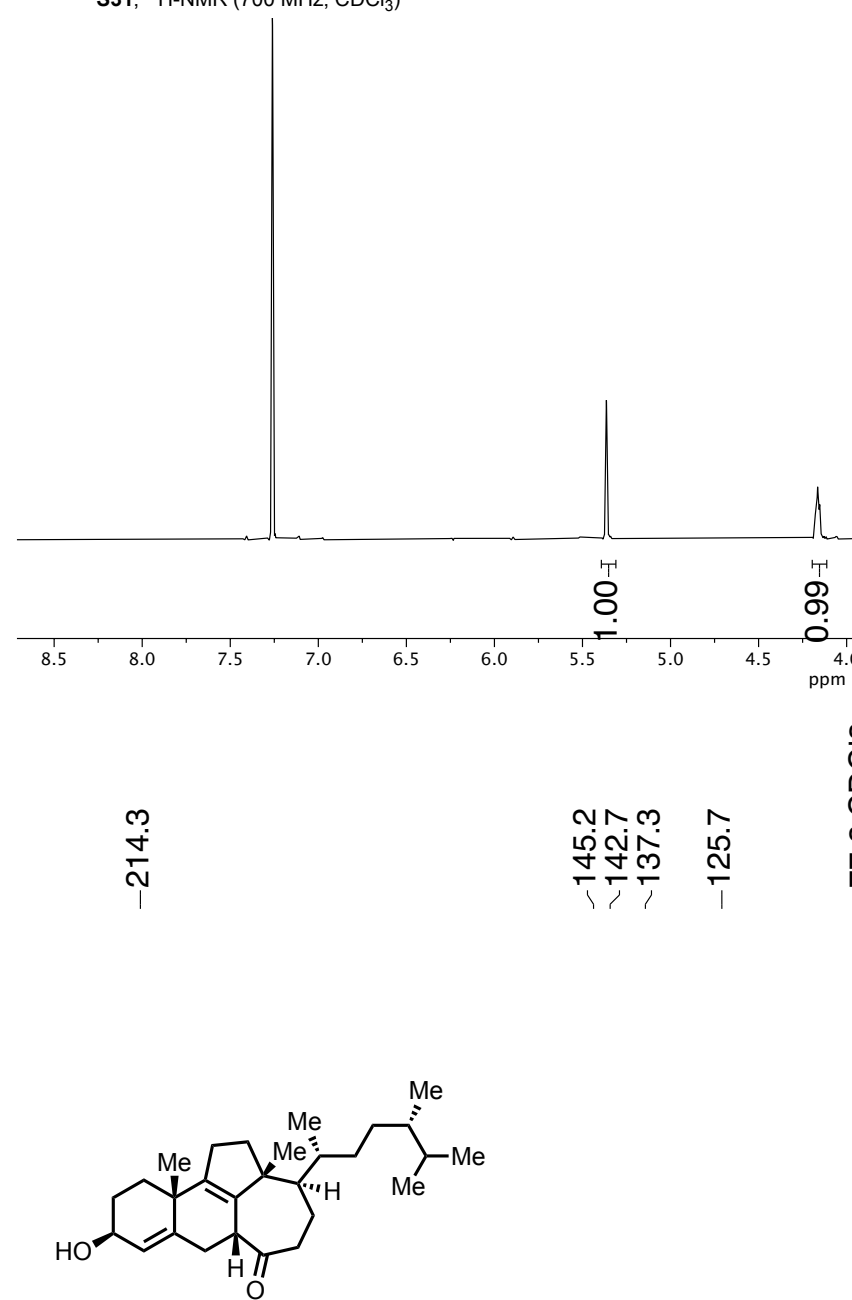

S31; ${ }^{13} \mathrm{C}-\mathrm{NMR}\left(101 \mathrm{MHz}, \mathrm{CDCl}_{3}\right)$

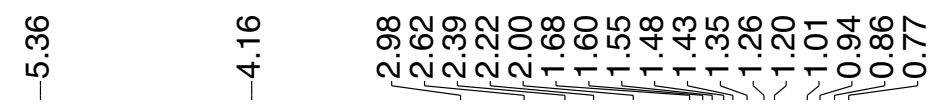

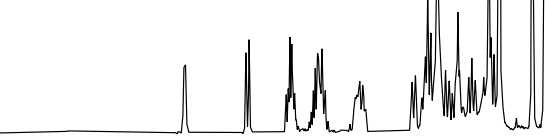

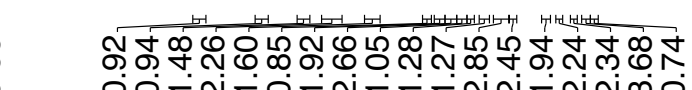

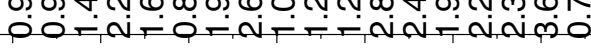

อุ

N $\infty$ -

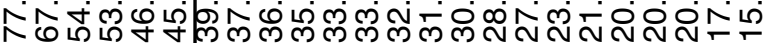

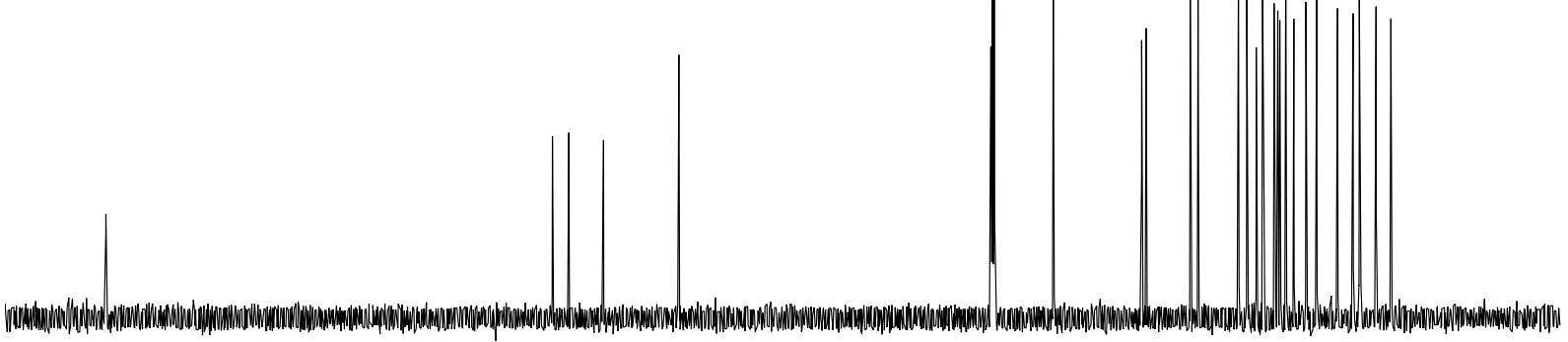

220 


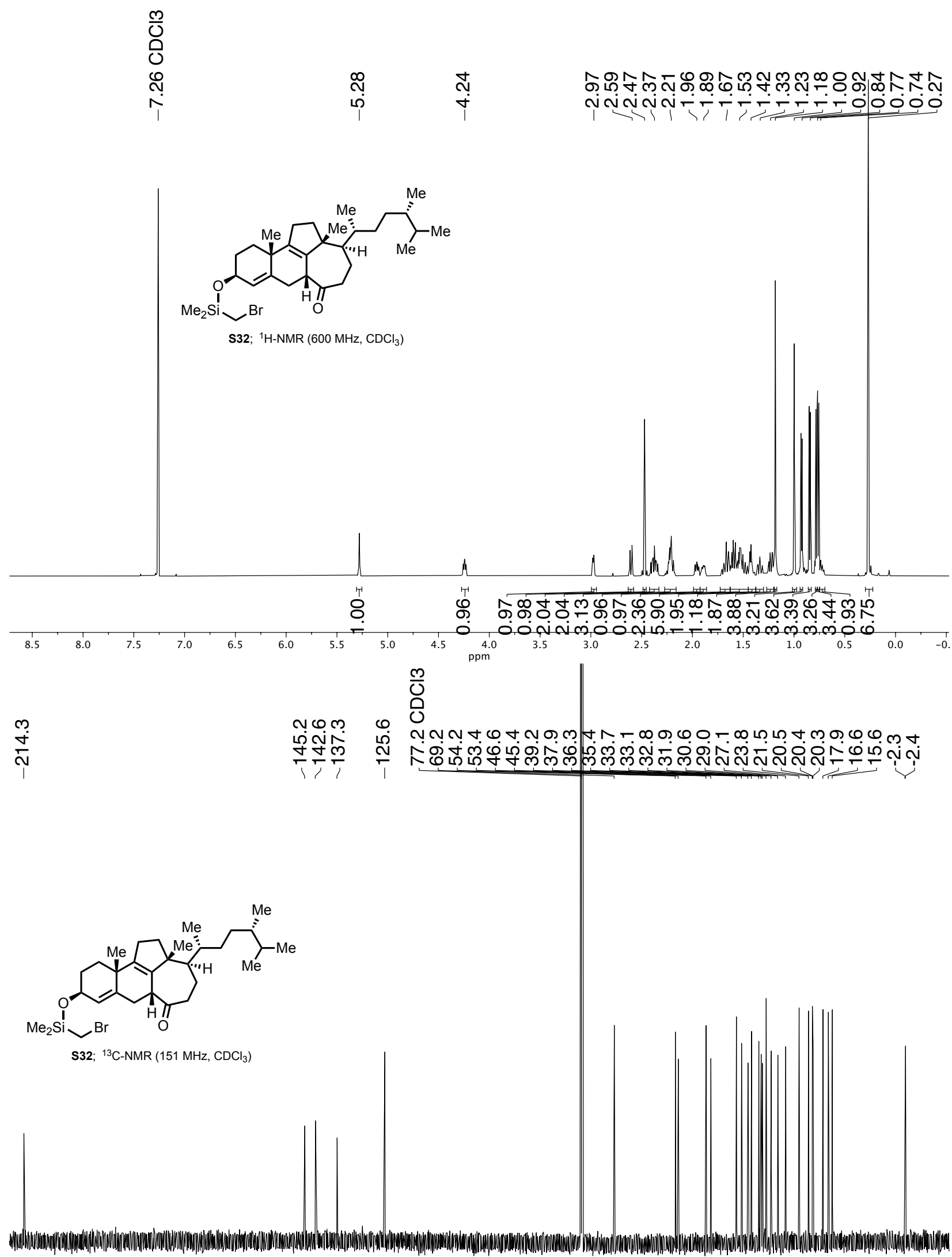

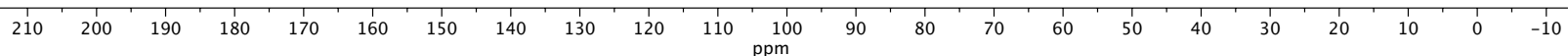




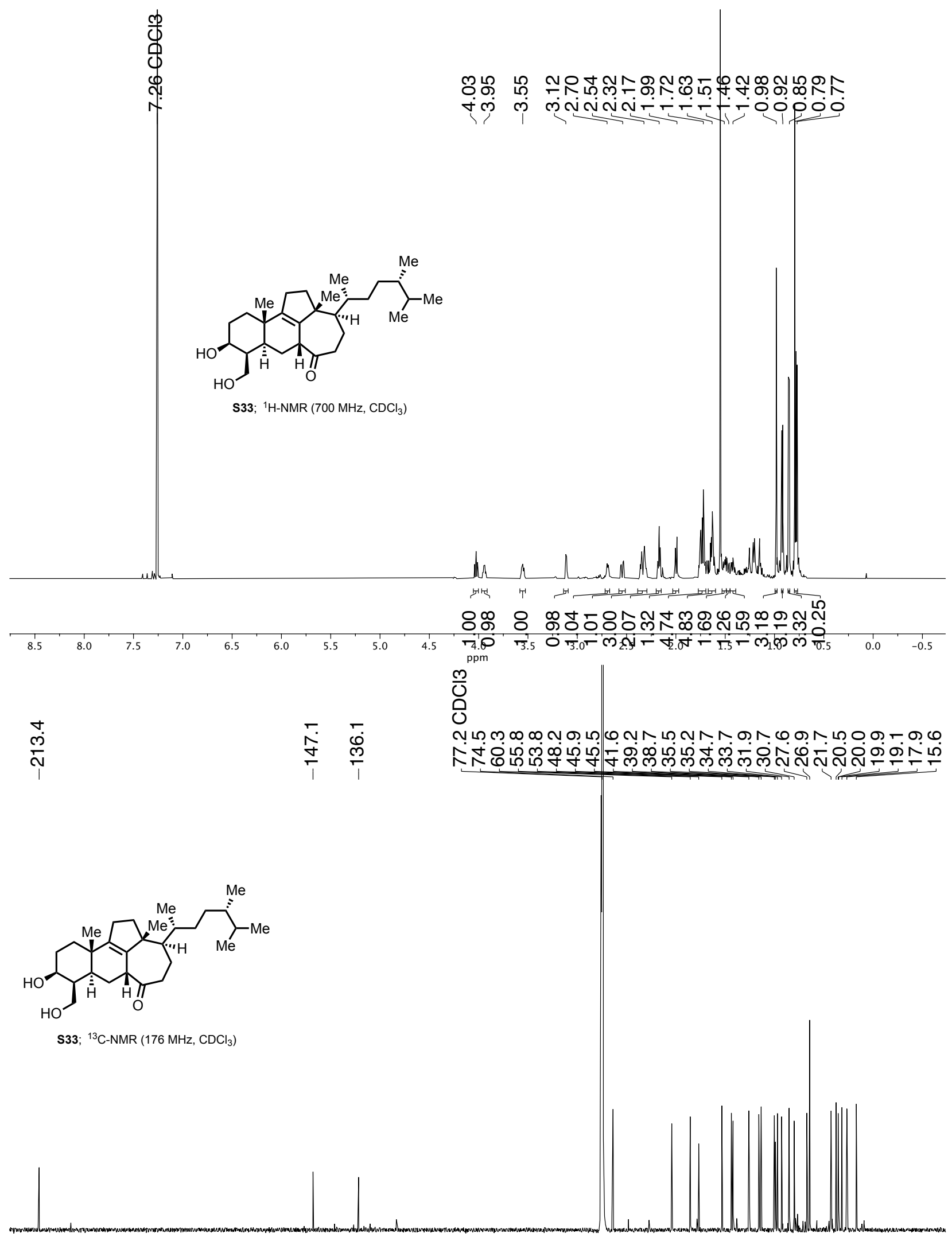

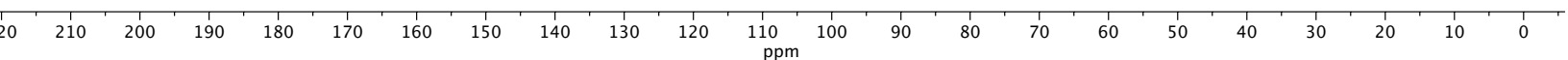




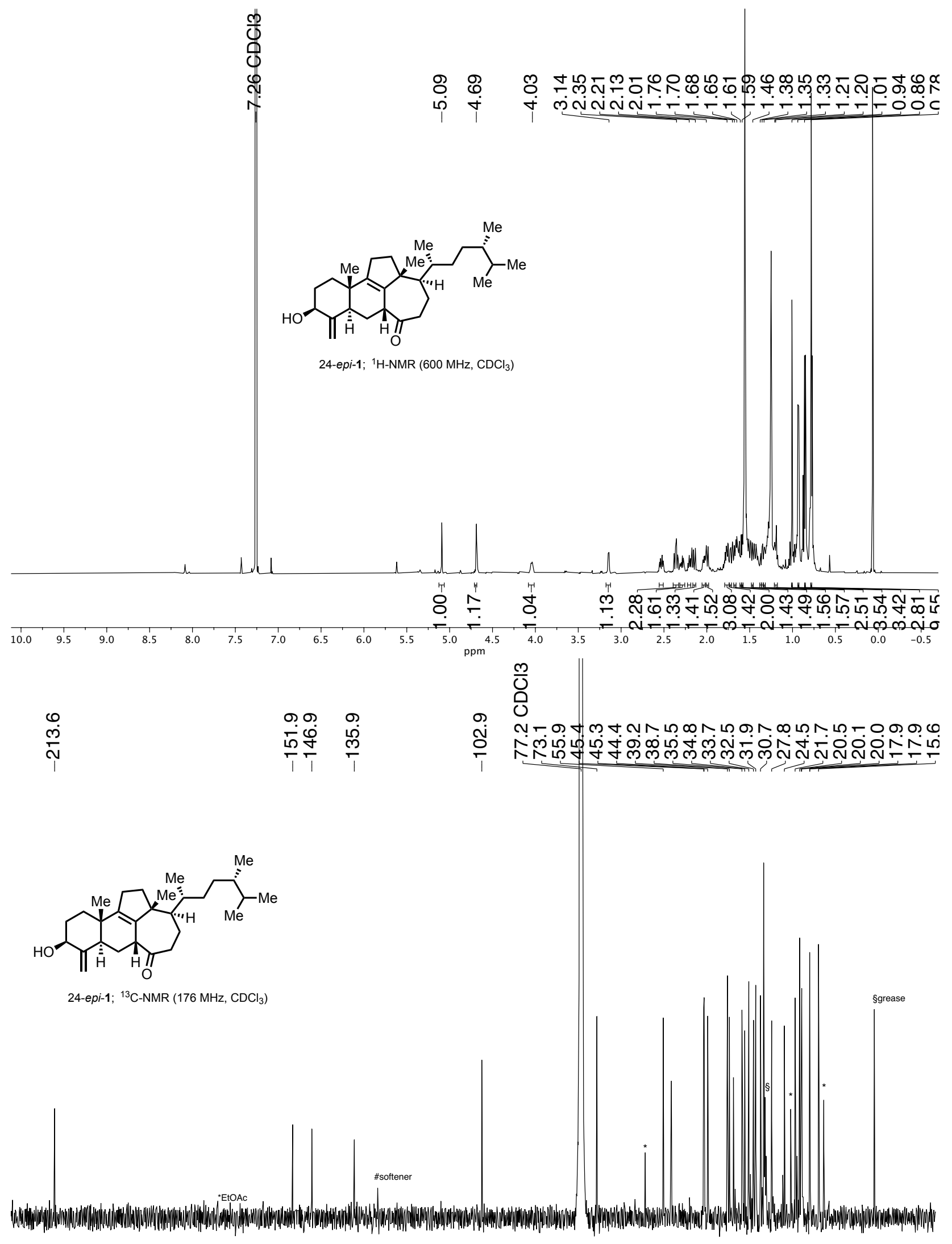

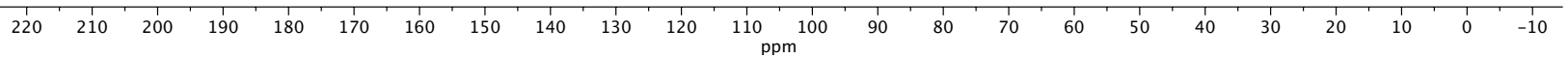




\section{X-Ray Crystallographic Data}

(22E)-3a,5-Cyclo-7a-iodo-13(14 $\rightarrow 8$ )abeo-5a-ergosta-22-en-6,14-dione (8)

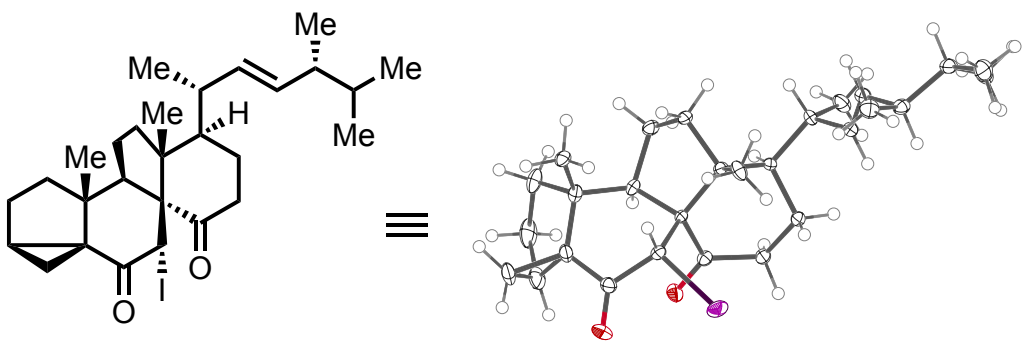

Table S6. Crystal data and structure refinement for 8.

Identification code

1900562

Empirical formula

$\mathrm{C}_{28} \mathrm{H}_{41} \mathrm{IO}_{2}$

Formula weight

536.54

Temperature/K

99.96

Crystal system

monoclinic

Space group

$\mathrm{P} 21$

$\mathrm{a} / \AA ̊ \AA$

9.8801(14)

$\mathrm{b} / \AA ̊ \AA$

11.6963(17)

c/Å

$11.5219(17)$

$\alpha /^{\circ}$

90

$\beta /{ }^{\circ}$

104.747(5)

$\gamma /{ }^{\circ}$

90

Volume/ $\AA^{3}$

1287.6(3)

Z

2

$\rho_{\text {calc }} \mathrm{g} / \mathrm{cm}^{3}$

1.3838

$\mu / \mathrm{mm}^{-1}$

1.265

$\mathrm{F}(000)$

555.4

Crystal size/mm

$0.8 \times 0.18 \times 0.03$

Radiation

Mo Ka $(\lambda=0.71073)$

$2 \Theta$ range for data collection $/{ }^{\circ} 4.86$ to 55.16

Index ranges

$-12 \leq h \leq 12,-15 \leq k \leq 15,-14 \leq \mathrm{l} \leq 14$

Reflections collected 33879

Independent reflections

$5901\left[R_{\text {int }}=0.0440, R_{\text {sigma }}=0.0322\right]$

Data/restraints/parameters

$5901 / 1 / 286$

Goodness-of-fit on $\mathrm{F}^{2}$

1.050

Final $R$ indexes $[\mathrm{I}>=2 \sigma(\mathrm{I})]$

$\mathrm{R}_{1}=0.0226, \mathrm{wR}_{2}=0.0463$

Final $R$ indexes [all data]

$\mathrm{R}_{1}=0.0277, \mathrm{wR}_{2}=0.0485$

Largest diff. peak/hole / e $\AA^{-3} \quad 0.71 /-0.45$

Flack parameter

$0.026(15)$ 

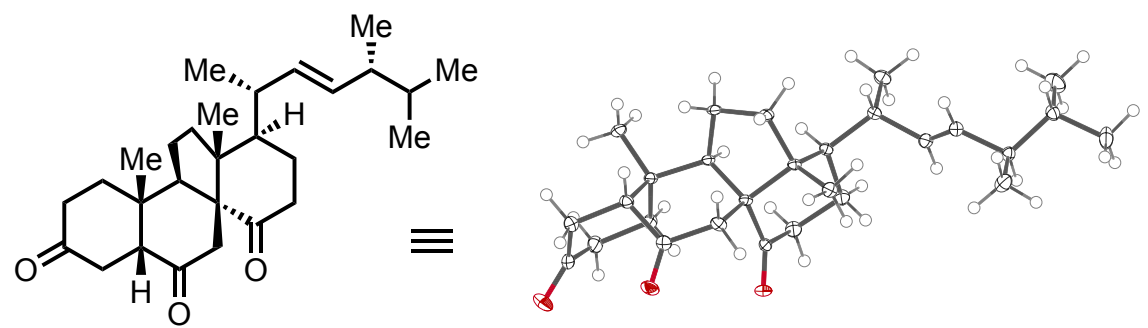

Table S7. Crystal Data and Structure Refinement for 2.

Identification code 1900563

Empirical formula

$\mathrm{C}_{28} \mathrm{H}_{42} \mathrm{O}_{3}$

Formula weight

426.61

Temperature/K

100.04

Crystal system

orthorhombic

Space group

$\mathrm{P} 2{ }_{1} 2_{1} 2_{1}$

$\mathrm{a} / \mathrm{A}$

11.238

$\mathrm{b} / \AA ̊ \AA$

11.741

c/Å

18.649

$\alpha /{ }^{\circ}$

90

$\beta /^{\circ}$

90

$\gamma /{ }^{\circ}$

90

Volume/Å ${ }^{3}$

2460.8

Z

4

$\rho_{\text {calc }} \mathrm{g} / \mathrm{cm}^{3}$

1.152

$\mu / \mathrm{mm}^{-1}$

0.072

$\mathrm{F}(000)$

936.0

Crystal size/mm ${ }^{3}$

$0.35 \times 0.18 \times 0.06$

Radiation

$\operatorname{MoKa}(\lambda=0.71073)$

$2 \Theta$ range for data collection/ ${ }^{\circ} 5.018$ to 61.236

Index ranges

$-16 \leq h \leq 16,-16 \leq k \leq 16,-26 \leq l \leq 26$

Reflections collected

56666

Independent reflections

$7551\left[R_{\text {int }}=0.0458, R_{\text {sigma }}=0.0302\right]$

Data/restraints/parameters

Goodness-of-fit on $\mathrm{F}^{2}$

$7551 / 0 / 286$

Final $R$ indexes $[\mathrm{l}>=2 \sigma(\mathrm{I})]$

1.140

Final $\mathrm{R}$ indexes [all data]

$\mathrm{R}_{1}=0.0537, \mathrm{wR}_{2}=0.1233$

Largest diff. peak/hole / e $\AA^{-3} \quad 0.73 /-0.33$

Flack parameter

$-0.1(3)$ 


\section{References}

(1) R. C. Heinze, P. Heretsch, Translation of a Polar Biogenesis Proposal into a Radical Synthetic Approach: Synthesis of Pleurocin A/Matsutakone and Pleurocin B, J. Am. Chem. Soc. 2019, 141, 1222-1226.

(2) R. C. Heinze, D. Lentz, P. Heretsch, Synthesis of Strophasterol A Guided by a Proposed Biosynthesis and Innate Reactivity, Angew. Chem., Int. Ed. 2016, 55, 11656-11659.

(3) T. Amagata, M. Tanaka, T. Yamada, M. Doi, K. Minoura, H. Ohishi, T. Yamori, A. Numata, Variation in Cytostatic Constituents of a Sponge-Derived Gymnascella dankaliensis by Manipulating the Carbon Source, J. Nat. Prod. 2007, 70, 1731-1740.

(4) T. Amagata, M. Doi, M. Tohgo, K. Minoura, A. Numata, Dankasterone, a new class of cytotoxic steroid produced by a Gymnascella species from a marine sponge, Chem. Commun. 1999, 14, 1321-1322.

(5) W. Gao, C. Chai, Y. He, F. Li, X. Hao, F. Cao, L. Gu, J. Liu, Z. Hu, Y. Zhang, Periconiastone A, an Antibacterial Ergosterol with a Pentacyclo[8.7.0.01,5.02,14.010,15]-heptadecane System from Periconia sp. TJ403-rc01, Org. Lett. 2019, 21, 8469-8472.

(6) J. Gong, P. Sun, N. Jiang, R. Riccio, G. Lauro, G. Bifulco, T.-J- Li, W. H. Gerwick, W. Zhang, New Steroids with a Rearranged Skeleton as (h)P300 Inhibitors from the Sponge Theonella swinhoei, Org. Lett. 2014, 16, 2224-2227. 